were to determine the: (1) agreement between MSI and MMR status (2) percent BRAF V600E IHC positivity (3) percent of MLH1 deficiency and (4) percent MLH1 deficient, BRAF V600E positivity.

Design: This study evaluated 204 consecutive colorectal cancer resections at single academic institution using FFPE tissues. The antibodies MLH-1 (M1), PMS2 (EPR3947), MSH2 (G219-1129) and CONFIRM MSH6 (44), and the mutation specific BRAF V600E (VE1) were run on the BenchMark XT automated platform (Ventana Medical Systems Inc. Tucson, Arizona). MSI status was determined by the MSI Analysis System (Version 1.2, Promega, Madison, WI), and BRAF V600E molecular status by the ABI 3730 (Life Technologies, Grand Island, NY).

Results: In this cohort, 83\% (169/204) were MSS, 17\% (35/204) were MSI-H, 84\% (172/204) were MMR proficient, 16\% (32/204) were MMR deficient, and 12\% (26/204) were MLH1 deficient. Average positive and negative agreement between MMR and MSI status (method of Cicchetti and Feinstein) was $95.5 \%$ and $99.1 \%$. Overall agreement was $98.5 \%$. Among BRAF IHC samples, $13.2 \%$ (27/204) were positive, $85.2 \%(174 / 204)$ were wildtype, and were 3 indeterminate. On repeat analysis, $2 / 3$ were reclassified as positive. Among MLH1 deficient samples, $54 \%$ (14/26) were positive and 46\% (12/26) were negative for BRAF V600E (VE1) IHC. Among the cases with both BRAF V600E IHC and sequencing results, 30/31 were in agreement: 13/13 negative agreement and $17 / 18$ positive agreement (1 was IHC positive, sequence negative).

Conclusions: This study demonstrated high overall agreement between MMR IHC and MSI, and the predominance of MLH1 deficiency. The BRAF V600E (VE1) IHC positivity rate (13.2\%) is comparable to the published mutation rate. As demonstrated in larger studies, there was a high overall agreement (30/31) for BRAF V600E status between Sanger sequencing and the VE1 antibody. Studies have also demonstrated a higher level of sensitivity for detection of BRAF V600E mutation by the BRAF V600E (VE1) antibody in comparison to some molecular methods including Sanger sequencing and PCR. A combination of the MMR and BRAF V600E (VE1) antibodies can be used effectively to aid sub-classification of colorectal carcinoma in the pathology laboratory.

865 Expression of Annexin A10 in Serrated Polyps Predicts the Development of Metachronous Neoplasia

G Zhang, $M$ Carole, R Lopez, P Carver, C Burke, R Pai. Cleveland Clinic, Cleveland, $\mathrm{OH}$.

Background: Sessile serrated adenomas/polyps (SSA/Ps) are precursors to colorectal carcinoma (CRC) whereas small distal hyperplastic polyps (HPs) are considered innocuous. Guidelines recommend a shorter screening interval for patients with SSA/Ps compared to diminutive distal HPs. Distinguishing SSA/Ps from HPs can be challenging. Previously we showed increased annexin A10 (ANXA10) expression in SSA/Ps compared to HPs suggesting that ANXA10 may be a marker of the serrated pathway to CRC. In this study we evaluate the ability of ANXA10 expression in baseline serrated polyps to predict the development of metachronous polyps.

Design: 52 patients undergoing a baseline colonoscopy with only serrated polyps were selected (38 patients with SSA/P and 14 patients with HP). Immunohistochemistry for ANXA10 (Novus biologicals 1:100) was performed on baseline polyps. Polyps were scored as having high (positive in $>50 \%$ of serrated crypts) or low expression (positive in $<50 \%$ of serrated crypts). Patients were divided into two groups: ANXA10 high and ANXA10 low. The development of metachronous SSA/Ps and adenomas on follow-up colonoscopy was assessed. Time of follow up was months from baseline colonoscopy to either first follow-up colonoscopy showing neoplasia or last available scope. ANOVA and univariable Cox regression were performed.

Results: 24 patients were in the ANXA10 low group (12 patients with SSA/Ps and 12 with HPs) and 28 patients in the ANXA10 high group (26 patients with SSA/Ps and 2 with HPs). Compared with the ANXA10 low group, the ANXA10 high group had a higher risk of developing SSA/Ps ( $25 \%$ vs. $4.2 \%$, $\mathrm{p}=0.068)$ and proximal adenomas $(28.6 \%$ vs. $8.3 \%, p=0.075)$ within 5 years. Importantly, high ANXA10 expression at baseline may be a better predictor of the development of SSA/Ps and adenomas than baseline histology (Fig 1).
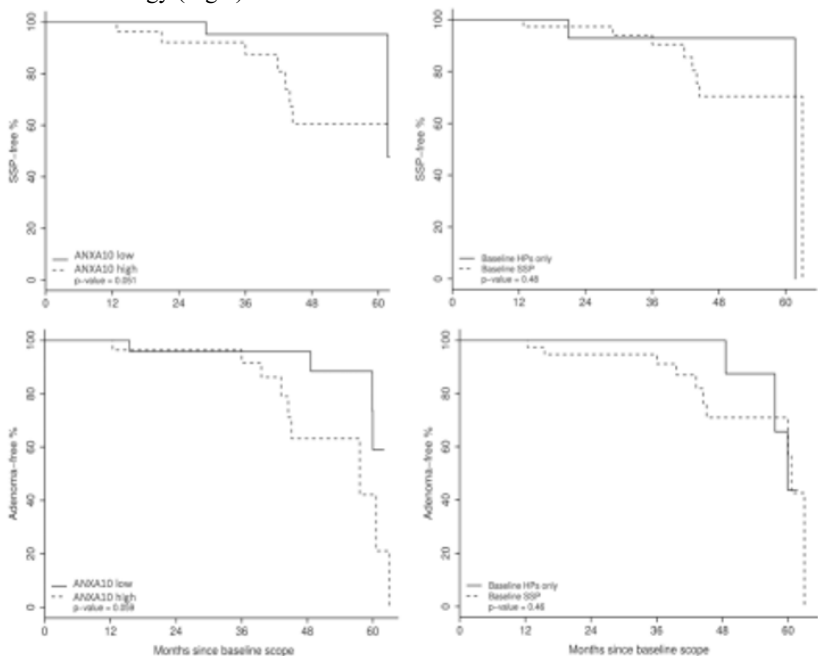

Conclusions: Our study suggests that ANXA10 expression in serrated polyps is a better predictor of the development of metachronous polyps than baseline polyp histology.
If confirmed in larger studies, this finding may impact surveillance for patients with serrated colorectal polyps.

866 MicroRNA Biomarker Differentiates Inflammatory Bowel Disease and Microscopic Colitis

ZZhao, H Osman, R Watson, I Nalbantoglu, M Bronner, J Lin. Indiana University School of Medicine, Indianapolis, IN; Washington University, St Louis, MO; University of Utah and ARUP Laboratories, Salt Lake City, UT.

Background: Idiopathic inflammatory bowel disease (IBD) and microscopic colitis (MC) are distinct entities. However, patients with intermittent episodes of IBD and MC that are encountered clinically puzzle clinicians and pathologists. Differential microRNA expression pattern has been reported in IBD. This study examines whether microRNA markers assist in the classification of patients with alternating episodes of IBD and MC. Design: MicroRNA was extracted from formalin-fixed, paraffin-embedded colon tissue and qRT-PCR was performed from cohorts of normal controls $(n=38)$, ulcerative colitis $(n=36)$, Crohn disease $(n=26)$, collagenous colitis $(n=36)$, lymphocytic colitis $(n=30)$, and the study group with intermittent features of IBD and MC $(n=5)$.

Results: Differential expression of miRNA-31 distinguished IBD (ulcerative colitis and Crohn disease) and MC (collagenous colitis and lymphocytic colitis), confirming the specificity of miRNA-31 in IBD $(P<0.05)$. In the study group, one patient had matching miRNA-31 expression pattern at different phases (lymphocytic colitis to ulcerative colitis, then back to collagenous colitis). The other 4 patients had MC-like expression patterns at both MC and IBD episodes.

Conclusions: In summary, miRNA-31 is a specific biomarker that differentiates between IBD and MC in formalin-fixed, paraffin-embedded colon tissue. The expression patterns of miRNA-31 in patients with intermittent IBD and MC are diverse. Although the underlying mechanisms remain unknown, microRNA biomarkers could be useful to characterize the individual patient with alternating episodes of IBD and MC.

\section{Genitourinary Pathology}

867 Prognostic Significance of RICTOR Protein Expression in Prostatic Adenocarcinomas (PACs): Nuclear Immunoreactivity Independently Predicts Disease Recurrence

KF Afaneh, OR Elkadi, JS Ross, GM Sheehan, CE Sheehan, HAG Fisher, RP Kaufman, BVS Kallakury. Albany Medical College, Albany, NY; Georgetown University Hospital, Washington, DC

Background: RICTOR is a core protein of the mTOR (mammalian target of rapamycin) pathway complex which plays a major role in cancer development and progression. Although RICTOR expression has been associated with breast cancer metastasis, its prognostic significance in prostate cancer has not been extensively studied.

Design: Formalin-fixed paraffin-embedded tissue sections from 114 PACs were immunostained by a manual method (DAKO LSAB + System-HRP) using mouse monoclonal RICTOR (ab56578; Abcam, Cambridge, MA). Cytoplasmic and/or nuclear immunoreactivity was scored based on intensity and percentage of positive cells in both the tumor and adjacent benign epithelium in each case. Cases were assessed as tumor $>$ benign $(\mathrm{T}>\mathrm{B})$, tumor=benign $(\mathrm{T}=\mathrm{B})$, and negative $(\mathrm{N})$. Results were correlated with clinicopathologic variables.

Results: Either cytoplasmic (cRICTOR) and/or nuclear (nRICTOR) immunoreactivity was noted for the tumor and adjacent benign glands in all cases. nRICTOR was increased in tumors as compared to benign glands [T>B 45\%, T=B 5\%, N 50\%], and correlated with tumor stage [T>B $59 \%$ adv vs $33 \%$ early, T=B $8 \%$ adv vs $3 \%$ early, N $33 \%$ adv vs $64 \%$ early; $\mathrm{p}=0.006]$ and biochemical disease recurrence [T $>\mathrm{B} 56 \%$ recur vs $34 \%$ non, $\mathrm{T}=\mathrm{B} 6 \%$ recur vs $5 \%$ non $\mathrm{N} 38 \%$ recur vs $61 \%$ non; $\mathrm{p}=0.045]$ ]. cRICTOR was also increased in tumors versus benign glands [T>B $61 \%, \mathrm{~T}=\mathrm{B} 19 \%, \mathrm{~N} 20 \%]$, and correlated with tumor grade [T>B $70 \% \mathrm{HG}$ vs $53 \% \mathrm{LG}, \mathrm{T}=\mathrm{B} 8 \% \mathrm{HG}$ vs $28 \% \mathrm{LG}, \mathrm{N} 22 \% \mathrm{HG}$ vs $19 \% \mathrm{LG} ; \mathrm{p}=0.025$ ] and tumor stage [ $\mathrm{T}>\mathrm{B} 63 \%$ adv vs $59 \%$ early, $\mathrm{T}=\mathrm{B} 10 \%$ adv vs $27 \%$ early, N $27 \%$ adv vs $14 \%$ early; $\mathrm{p}=0.033$. On multivariate analysis, nRICTOR independently predicted biochemical disease recurrence $(\mathrm{p}=0.03)$.

Conclusions: Both nuclear and cytoplasmic RICTOR expression are significant biomarkers of adverse prognosis in prostate cancer. Nuclear RICTOR expression is an independent predictor of biochemical disease recurrence. Further study of RICTOR expression and its potential role in prostate cancer appears warranted.

868 Inverted/Endophytic Growth Pattern of Papillary Urothelial Neoplasms of the Bladder: Clinicopathological Study in 225 Transurethral Resection Specimens

AS Akki, K Richards, ND Smith, GD Steinberg, GP Paner. University of Chicago, Chicago, IL.

Background: Inverted growth in papillary urothelial neoplasm (PUN) is a wellrecognized morphologic variation that may mimic invasive urothelial carcinoma. The clinical significance of inverted growth in PUN is unclear, and to date, no large clinicopathological study has investigated PUNs with inverted growth pattern.

Design: A histopathological review of 259 bladder transurethral resection (TUR) specimens (from 115 patients) from a single tertiary institution was conducted. Of these, 225 TURs contained PUNs that were included in the study. 34 TURs with CIS, urothelial papilloma or other benign lesions (from 14 patients) were excluded. Inverted growth was defined as non-invasive intrusion of PUN into the lamina propria. The inverted component within a PUN was quantified after delineating the surface and exophytic papillary components (i.e. non-inverted parts). Patients were followed for recurrence from first presentation (initial TUR, 1 month to 7 years follow-up). Correlation analysis was performed by Fischer's exact test. 
Results: Any inverted growth (focal or diffuse) was identified in 68/225 (30\%) PUNs and occurred in 41/102 (40\%) patients when all TURs were accounted for. Mean patient age was 71.5 years (range 49-93 years) for PUNs with inverted component and 70.3 years (range 46-93 years) for pure non-inverted PUNs. Inverted growth was seen in PUN of low malignant potential $(1 / 68,1.5 \%)$, low grade $(25 / 68,37 \%)$ and high grade $(42 / 68,61.5 \%)$ papillary carcinomas, and overall comprised $25 / 114(22 \%)$ of low grade and $42 / 130(32 \%)$ of high grade papillary carcinomas. The extent of inverted growth in a tumor varied and was $\leq 10 \%(19 / 68,28 \%), 11-29 \%(17 / 68,25 \%)$ and $\geq 30 \%(32 / 68$, $47 \%$ ) of the PUNs. Patterns of inverted growth included trabecular, bulbous, inverted papilloma-like (i.e. peripheral nuclear palisading but with broad growth), focal trabecular and mixed types. No correlation was found between the presence of inverted component and histologic grade. On follow-up, there was no correlation between the presence of inverted component and recurrence.

Conclusions: Inverted growth pattern is seen in a minority of PUNs and our findings suggest no correlation of the inverted growth pattern with the histologic grade or tumor recurrence.

\section{Cysteine- Rich Secretory Protein 3 (CRISP3), ERG and PTEN Define a Molecular Subtype of Prostate Cancer with Poorest Clinical Patient's Outcome}

S Al-Bashir, M Alshalalfa, S Hegazy, M Dolph, B Donnelly, T Bismar. Jordan University of Science and Technology, Irbid, Jordan; University of Calgary, Calgary, AB, Canada; University of Calgary and Calgary Laboratory Services, Calgary, AB, Canada.

Background: Cysteine- rich secretory protein 3 (CRISP3) prognostic significance in prostate cancer (PCA) has generated mixed result. We investigated and independently validated CRISP3 expression in relation to ERG and PTEN genomic aberrations and clinical outcome.

Design: CRISP3 protein expression was examined by immunohistochemistry using a cohort of 215 patients with localized PCA and castrate resistant PCA (CRPC) and lined to several clinical-pathological parameters including ERG and PTEN genomic aberrations. The Memorial Sloan Kettering (MSKCC) and Swedish cohorts were used for prognostic validation.

Results: CRISP3 intensity was significantly associated with neoplastic epithelium, being highest in CRPC compared to benign prostate tissue $(\mathrm{p}<0.0001)$. CRISP3 showed insignificant trends in relation to Gleason score by immunohistochistry and was significantly associated with high Gleason score at mRNA levels $(\mathrm{p}=0.022$ in MSKCC, $\mathrm{p}=1.1 \mathrm{e}-4$ in Swedish). Significant association between CRISP3 expression and clinical outcome was documented on the mRNA levels but not at the protein expression level. CRISP 3 mRNA expression was related to biochemical recurrence $(\mathrm{p}=0.038)$ and lethal disease $(\mathrm{p}=0.0086)$ in the MSKCC and the Swedish cohorts respectively, and retained its prognostic value in the subgroup of patients with GS 6 and 7 characterizing CRISP3 expression relative to PCA molecular aberrations. CRISP3 expression was significantly associated with positive $E R G$ status $(\mathrm{p}=0.002)$ and with PTEN deletions $(\mathrm{p}=0.005)$ Overexpression of CRISP 3 was also associated with positive $E R G(\mathrm{p}=2.9 \mathrm{e}-9$ in MSKCC, $\mathrm{p}=2.5 \mathrm{e}-7$ in Swedish) and with PTEN deletion $(\mathrm{p}=0.05)$. Furthermore, functional biology analysis documented phenylalanine metabolism as the most significant pathway governing high CRISP3 and $E R G$ expression in this subtype of PCA

Conclusions: The combined status of CRISP3, ERG and PTEN define a molecular subtype of PCA with poorest and lethal outcome. Our findings support a prognostic role of CRISP3 linked to molecular subtype of PCA. Further work assessing the combined value of CRISP3, ERG and PTEN may be of added value in stratifying PCA patients into different prognostic group and to identify patients with poorest clinical outcome.

870 The Expression Patterns of p53 and p16 and an Analysis of a Possible Role of HPV in Primary Adenocarcinoma of the Urinary Bladder RE Alexander, JD Richey, SR Williamson, A Lopez-Beltran, R Montironi, DD Davidson, MT Idrees, CL Jones, S Zhang, L Wang, Q Rao, GT MacLennan, L Cheng. Indiana University School of Medicine, Indianapolis, IN; Henry Ford Health System, Detroit, MI; Cordoba University, Cordoba, Spain; Polytechnic University of the Marche Region and United Hospitals, Ancona, Italy; Nanjing Jinling Hospital and Nanjing University School of Medicine, Nanjing, China; Case Western Reserve University, Cleveland, $\mathrm{OH}$ Background: Primary adenocarcinoma of the urinary bladder is rare. The molecular and cellular events leading to its pathogenesis are not well delineated. The cell cycle proteins, p53 and p16, have been extensively studied in a wide variety of human malignancies, but investigation of both markers in a single study has not been performed in urinary bladder adenocarcinoma. The goal of this study was to investigate p53 and p16 expression, as well as HPV status, in a relatively large series of primary bladder adenocarcinomas. Design: Thirty-seven cases of urinary bladder adenocarcinoma were chosen from participating institutions. Diagnosis and available clinical history was reviewed in each case. Immunostainings for p53, p16 and HPV and high-risk and low-risk HPV-ISH were performed on all cases.

Results: Patients had an average age of 61 years with a male predominance (1.5: male:female ratio). The average tumor size in cystectomy specimens was $4.3 \mathrm{~cm}$. Nearly half of cases were transurethral resections or biopsies, limiting accurate assessment of maximum tumor dimension. Of the cases managed by transurethral resection, $40 \%$ were pT2 at the time of diagnosis. $77 \%$ of cystectomy specimens were either pT3 or pT4. Strong nuclear p16 expression was seen in 76\% (28 of 37) of cases. p53 expression was seen in $60 \%$ ( 22 of 28 ) cases. Expression of both markers was seen in $41 \%$ (15 of 37) of cases. Expression of p16 or p53 alone was seen in $13(35 \%)$ and $7(19 \%)$ cases, respectively. In only $2(5 \%)$ cases, neither marker was expressed. No significant correlation between clinical variables and any of the markers we studied was identified. HPV infection was not detected in any case.
Conclusions: Expression of p53 and/or p16 is a very common finding in urinary bladder adenocarcinoma. These findings implicate a high likelihood that alterations in these cell cycle proteins contribute to the pathogenesis of these tumors. Despite the high prevalence of p16 staining seen, no evidence of HPV infection was found and it should not be interpreted as a surrogate for HPV in these tumors.

\section{Next Generation Targeted Prostate Biopsies vs. Standard Multicore} Prostate Biopsies: Comparing Diagnostic Yield

MN Al-Kawaaz, CS Friedman, MM Shevchuk. NYP-Weill Cornell Medical College, New York, NY

Background: Targeted prostate biopsies using transrectal ultrasound (TRUS) guided by pre-biopsy MRI findings have emerged as a promising technology for prostate cancer ( $\mathrm{PCa}$ ) detection. The Artemis system, recently approved by the FDA, is one such MRI-TRUS fusion device. In this pilot study, we compared Artemis-guided prostate biopsies with the standard 10 to 14-core biopsies for PCa detection, and for evaluation of Gleason grade and tumor volume. Can the more limited targeted biopsy augment or even replace the standard biopsy?

Design: Thirty-six patients underwent an Artemis-targeted biopsy (4-8 cores) in combination with the standard 10 to 14-core biopsies, which were performed either at the same time, or within 6 months of the Artemis biopsy. The results were evaluated by genitourinary pathologists.

Results: Twenty-three of 36 patients $(64 \%)$ were found to have PCa. In 11 of these cases (31\%), both Artemis-guided and standard biopsies detected PCa. In 5 cases (14\%), only the Artemis-guided biopsy detected PCa; and in another 7 cases (19\%), only the standard biopsies detected $\mathrm{PCa}$. Benign findings were present in 13 cases $(36 \%)$ by both Artemis and standard biopsies. As to Gleason grade, Artemis-guided biopsies detected higher grade disease. Of the five cases of PCa detected only by Artemis, two cases showed Gleason 4+3, one case showed Gleason 3+4, and two cases showed Gleason 3+3. On the other hand, out of the seven cases of PCa detected only by standard biopsy, six showed Gleason 3+3, and one showed Gleason 3+4. Furthermore, in cases in which both Artemis-guided and standard biopsies identified PCa, 5 of 11 were underestimated (lower Gleason grades or lesser tumor volume) by the standard biopsies; and 2 of 11 were underestimated by the Artemis-guided biopsies.

Conclusions: Artemis-guided biopsies detected $14 \%$ of the PCa which were missed by standard biopsy; most of these tumors having high Gleason grades. Another 14\% of the detected PCa were underestimated by standard biopsy alone. Of the $19 \%$ of the PCa that were missed by Artemis-guided biopsies, most were low grade. An additional $6 \%$ were underestimated by Artemis alone. Therefore Artemis-guided biopsies outperformed standard biopsies in identifying more significant cancers. Adding Artemis-guided biopsies to the standard protocol would increase the diagnostic yield and grading accuracy. Currently, Artemis-guided biopsy probably should not replace standard biopsies. Further improvements in this technology show promise to change prostate biopsy practice in the future.

\section{Clinicopathological Analysis of Testicular Germ Cell Tumor (GCT)} with Rhabdomyosarcomatous Transformation

I Alvarado-Cabrero, N Hernandez-Toriz, GP Paner. Mexico Oncology Hospital, IMSS, Mexico City, Mexico; University of Chicago, Chicago, IL.

Background: Development of sarcomatous components in GCTs is rare with rhabdomyosarcoma (RMS) being the most frequent type. While studies of combined gonadal and extragonadal GCTs suggest an aggressive behavior for sarcomatous transformation, data exclusively derived from testicular GCTs with RMS component has been limited. This study focused only on testicular GCTs with RMS component treated at a single institution.

Design: A comprehensive review of histology slides from 1,010 tumoral orchiectomies from the surgical pathology files (1999-2011) of a national oncology institute was conducted. 20 (2\%) GCT with RMS cases were identified, which formed the basis of this study. IHC for desmin and myogenin were performed on select cases for confirmation. Results: Patient age ranged from 19 to 42 years (mean 31 years). Stage at presentation was II ( $80 \%)$ and III (20\%). The RMS consisted $30 \%$ to $90 \%$ of the testicular tumor and typically showed high grade undifferentiated small blue or spindled cells with rhabdomyoblasts. Three testicular tumors had also PNET and undifferentiated spindle cell sarcoma components. The non-transformed testicular tumor showed mixed (55\%) or single (45\%) GCT element, most commonly teratoma (75\%), seminoma $(40 \%)$ and yolk sac tumor $(35 \%)$. Lymphovascular, tunica vaginalis and spermatic cord invasion were seen in $40 \%, 55 \%$, and $35 \%$ of cases, respectively. All 20 patients had retroperitoneal lymph node metastasis, of which $9(45 \%)$ contained RMS mostly combined with GCT elements (RMS range $40-100 \%$ of metastatic tumor) and $1(5 \%)$ contained PNET. Follow-up available in 19 patients (range 12-84 months, median 48 months) showed 9 $(47 \%)$ dead of disease, $2(10 \%)$ alive with disease and $8(42 \%)$ alive without disease. The 5-year disease specific survival was $31 \%$. Of the 9 deaths, 7 had RMS and 1 had PNET in the retroperitoneal metastasis. In contrast, only 1 of 10 patients with nonsarcomatous retroperitoneal metastasis died of the disease. Distant metastasis involved the lungs (2) and liver (1)

Conclusions: This series, the largest to date, confirmed the aggressive behavior for testicular GCT with RMS component. However, stage II GCTs with RMS but localized to testis may behave less aggressively. Presence of RMS in retroperitoneal metastasis portends a worst prognosis. 


\section{Pathological Features of Metastatic Regional Lymph Nodes in} Penile Squamous Cell Carcinoma

I Alvarado-Cabrero, DF Sanchez-Martinez, $R$ Valencia-Cedillo, JM Gomez-Cifuentes, M Suarez-Perez, AL Cubilla. Mexican Oncology Hospital, Mexico, DF, Mexico; Universidad Nacional, Asunción, Paraguay; Hospital Universitario San José, Bogotá, Colombia; Hospital San Juan de Dios, San José, Costa Rica; Instituto de Patología e Investigación, Asunción, Paraguay.

Background: There are few comprehensive pathological evaluation of metastatic inguinal lymph nodes in patients with penile squamous cell carcinoma. The aim of the study was to identify factors influencing outcome.

Design: 126 penectomy specimens and bilateral groin dissections from the Mexican Oncology Hospital, were evaluated. There were 4 to 36 lymph nodes per case. Factors evaluated were: number of nodes involved, tumor extension within the nodes, histological type of primary vs metastatic node, histological grade in the primary as well as in the metastatic node. Also evaluated were the pathological features of tumors of patients surviving despite presence of nodal metastasis. Follow up ranged from 11 to 82 months Statistical analysis was done with Fisher's exact test for categorical data, Kruskal-Wallis for continuous data, Kaplan-Meyer for survival analysis.

Results: There was a high survival rate of patients with 1 metastatic node (86\%), intermediate in those with $2(53 \%)$ and poor survival in those with 3 positive nodes $(26 \%)$. Micrometastasis, macrometastasis and confluent metastasis were associated with low (25\%), intermediate (55\%) and high mortality (73\%). Metastatic and primary tumors were morphologically similar, with minor variation $(90 \%$ agreement, kappa: 0.86). Similarly, histological grade was homogenous in primary and metastatic tumors with minimal deviation ( $90 \%$ agreement). Evaluating pathological factors in 9 patients surviving despite presence of nodal metastasis we found their primary tumors invading beyond corpus spongiosum in only a third of the cases (33\%), their thickness averaged $12 \mathrm{~mm}$, had frequent vascular invasion $(89 \%)$ and high grade $(76 \%)$ but perineural invasion was infrequent $(22 \%)$.

Conclusions: Histological types and grades were similar in primary and metastatic carcinomas. Primary tumors of surviving patients were more likely to be more superficial, invading up to corpus spongiosum with infrequent perineural invasion. Patients dying from disease had deeply invasive tumors in corpora cavernosa and frequent perineural invasion. Metastasis to one inguinal lymph node was associated with a good survival whereas high rates of mortality were found in tumors involving more than 2 lymph nodes.

\section{SALL4 Is a Better Marker Than PLAP in Metastatic Treated} Malignant Germ Cell Tumors

NK Andeen, MS Tretiakova. University of Washington, Seattle, WA

Background: Studies have shown that in the metastatic setting and after treatment, expression of immunohistochemical markers of germ cell origin is often diminished or lost. Transcription factor SALL4, which maintains embryonal stem cells, has been recently recognized as a very sensitive and specific marker for both primary and metastatic malignant germ cell tumors (MGCT) (PMID: 19574883; PMID: 23224120). However, SALL4 has not been tested in the post-treatment setting. We sought to determine the level of SALL4 expression in treatment-resistant metastatic MGCT in comparison with pan-GCT marker placental alkaline phosphatase (PLAP).

Design: Forty cases of lymph node $(n=31)$ or visceral metastases $(n=9)$ of MGCT that had been treated by chemo- and/or radiation therapy were immunostained for SALL4 and PLAP. For each MGCT component an H-score was calculated (sum of the multiplicands of the percentage of cells staining at each intensity level (scale $0-3$ ), by the intensity level value).

Results: There was intense SALL4 nuclear immunoreactivity in the majority of cells (mean H-score 260) in 31/40 cases (78\%), labeling seminoma, yolk sac, embryonal, and peripheral neuroectodermal (PNET) components. In 7/9 cases with a SALL4 $\mathrm{H}$-score $<100$, the predominant component was mature or immature teratoma, wherein SALL4 was only focally expressed in the epithelial component. In the other 2 cases with focal SALL4 staining (H-score $<100)$, the components were choriocarcinoma and osteosarcoma. No treated metastatic MGCT lacked SALL4 immunoreactivity. In contrast, PLAP was expressed in the majority of cells (mean H-score 245) in 18/40 $(45 \%)$ cases of MGCTs, and showed scattered variable positivity (H-score $<100)$ in 13/40 $(32 \%)$ cases, including 7 teratomas and 6 non-teratomas. PLAP was virtually absent (H-score $<10)$ in $9 / 40(23 \%)$ cases, including 7 MGCTs with yolk sac, embryonal, poorly differentiated carcinoma, and PNET components, and 2 teratomas.

Conclusions: SALL4 has strong diffuse nuclear reactivity in the majority of MGCT components in the post-treatment setting, and is a more sensitive marker than PLAP, which had variable or absent expression in a significant number of cases. In cases where SALL4 expression was focal, PLAP was either absent (choriocarcinoma), or focal and weak (teratomas). Persistent expression of SALL4 in metastatic MGCTs that are resistant to chemoradiation raises the possibility of it being a target for systemic therapy as the anti-SALL4 peptide continues to be developed.

875 Utility of ERG vs. AMACR Expression in Diagnosis of Minimal Adenocarcinoma of the Prostate in Needle Biopsy Tissue

CG Andrews, PA Humphrey. Washington University, Saint Louis, MO.

Background: Prostate adenocarcinoma measuring less than $1 \mathrm{~mm}$ presents a diagnostic challenge due to the limited number of glands present for evaluation. The $\alpha$-MethylacylCoA Racemase (AMACR) immunostain, a marker of neoplastic prostatic epithelial cells, may be used to evaluate these limited tumor cases, in needle biopsy, with a sensitivity ranging to a low of $80 \%$. The use of the ERG immunostain in evaluating prostate cancer is becoming more common, but the examination of the utility of this marker in direct comparison to AMACR has not been performed. The purpose of our study was to investigate whether the ERG immunostain adds diagnostic value to AMACR alone in evaluating untreated prostate cancer foci measuring less than $1 \mathrm{~mm}$ on core needle biopsy.

Design: We identified 131 blocks from 114 patients with continuous tumor foci measuring less than one mm on core needle biopsy. ERG and AMACR immunostains were performed on serial sections from the blocks, and expression was assessed by intensity and proportion scores assigned to each stain.

Results: 66 of the selected blocks from 64 patients retained tumor foci measuring less than $1 \mathrm{~mm}$ after obtaining deeper sections. Of these 66 tumor foci, 37 were positive for AMACR alone, 28 were positive for AMACR and ERG, and 1 was positive for ERG alone. AMACR had a sensitivity of $98.5 \%$ and ERG had a sensitivity of $43.9 \%$. Most cases displayed strong AMACR expression, and only 7 of 66 blocks (10.6\%) exhibited weak or negative AMACR expression. Of these 7 cases with weak or negative AMACR expression, only 2 cases were ERG positive.

Conclusions: This is the first study to our knowledge that examines the diagnostic utility of ERG expression in comparison to AMACR expression in minimal usual acinar adenocarcinoma of the prostate on core needle biopsy. Our findings suggest that AMACR should be the first line marker for confirmation of a diagnosis of minimal adenocarcinoma of the prostate, when needed. ERG immunohistochemistry is indicated only in uncommon cases of minimal adenocarcinoma when AMACR staining is negative or weak. Evidence-based utilization of diagnostic markers, without routine over-utilization of markers, such as ERG expression in minimal adenocarcinoma, that do not provide added diagnostic value in most cases, is an important principle in this era of cost-consciousness in application of immunohistochemistry.

876 Does Discontinuous Involvement of a Prostatic Needle Biopsy Core by Adenocarcinoma Correlate with the Presence of a Large Tumor Focus at Radical Prostatectomy?

JA Arias-Stella, K Varma, D Montoya-Cerrillo, NS Gupta, SR Williamson. Henry Ford Health System, Detroit, MI; Universidad Peruana Cayetano Heredia, Lima, Peru.

Background: When prostate core needle biopsies are involved discontinuously by tumor (benign tissue intervening between two or more foci of cancer), there remains no consensus on the optimal method of tumor quantitation. Some data indicate that including the benign intervening prostatic tissue in the measurement correlates better with stage and margin status than subtracting it. However, since prostate cancer is characteristically multifocal, it is possible that this phenomenon sometimes results from multiple clinically insignificant tumor foci. We investigated whether discontinuous core biopsy involvement typically results from a single, large tumor focus or multiple small foci.

Design: Prostate core needle biopsies noted to have discontinuous tumor and the corresponding whole-mounted radical prostatectomies from 2008-13 were retrieved via database search. Pairs were included in analysis only if the discontinuously positive core had the largest percentage of involvement of all cores and if there was a distance of at least $2 \mathrm{~mm}$ between discontinuous foci. Linear length and percentage of biopsy involvement were measured both including and subtracting the benign intervening tissue. The corresponding region of the radical prostatectomy specimen was evaluated for tumor size and multifocality.

Results: Over 800 core needle biopsies were performed annually during the study period; however, only 26 patients met the above criteria for study inclusion and underwent radical prostatectomy. Subtracting the benign intervening tissue, length and percentage of biopsy involvement ranged from 1-5 mm and 5-50\% (median $2 \mathrm{~mm}$ and $20 \%$ ), whereas including the intervening tissue yielded a range of $4-15.5 \mathrm{~mm}$ and $30-100 \%$, (median $7 \mathrm{~mm}$ and $72.5 \%$ ). The largest gap of intervening tissue ranged from 2-10.5 mm (median $3.5 \mathrm{~mm}$ ). In 19 cases (73\%), a single tumor focus, often irregularly shaped, was present in the corresponding anatomic region of the prostate. The same focus was also the dominant tumor in $17 / 19$. In the remaining 7 cases, multiple small tumor foci were present in the corresponding region of the prostate.

Conclusions: Discontinuous tumor in a prostatic core needle biopsy specimen usually, but not always, results from a single tumor focus in the corresponding region of the prostate gland. Therefore, we recommend that such involvement be noted in the pathology report and differentiated from a small tumor focus (subtracting the benign intervening tissue) and a large uninterrupted focus.

877 Composite Gleason Score (CGS) Is Superior to Grading Individual Needle Cores When More Than Two Grades Are Present in a 12 Core Prostate Biopsy: A Study of 118 Cases

JA Arias-Stella, R Gulati, D Montoya-Cerrillo, SR Williamson, NS Gupta. Henry Ford Health System, Detroit, MI; Universidad Peruana Cayetano Heredia, Lima, Peru.

Background: The 2005 ISUP consensus recommends grading individual needle cores when they are separately identified in multiple containers. Urologists typically select the core with highest Gleason score (HGS) as the grade of the entire case though a lower grade may occupy most of the biopsy tissue. This technique may falsely place the patient in a higher prognostic group or make him an unsuitable candidate for certain treatment modalities. We aim to compare CGS versus HGS and determine which method is more accurately predictive of final radical prostatectomy (RP) Gleason score (GS). Design: Prostate needle core biopsies performed between 2008 and 2012 with $>2$ different GS and corresponding RP were selected. Variables included highest and lowest biopsy GS, number and location of positive cores, percentages of patterns 3, 4, and 5, RP GS, location of dominant nodule, stage, and age. CGS was assigned by taking into account all contiguous positive needle biopsies, volume of each positive core and percentages of each Gleason pattern. CGS was compared with HGS and RP GS. Categories of GS were divided according to Prognostic groups (PGr) I to V to define downgrade/upgrade in GS. 
Results: We analyzed 1193 biopsies, of which 197 met our criteria. Follow up RP was available in 118 cases. Correlation of CGS and HGS with RP GS is shown in the table below.

Correlation of CGS \& HGS with RP GS

\begin{tabular}{|l||l|l|}
\hline \hline & CGS vs RP GS & HGS vs RP GS \\
\hline Downgraded & $14(11.9 \%)$ & $80(67.8 \%)$ \\
\hline Same & $61(51.7 \%)$ & $26(22.0 \%)$ \\
\hline Upgraded & $43(36.4 \%)$ & $12(10.2 \%)$ \\
\hline
\end{tabular}

Downgrade rates for the two methods are shown in the following table.

Downgrade rates for CGS and HGS

\begin{tabular}{|l|l|l||}
\hline \hline & 1 PGr downgrade & 2 or more PGr downgrade \\
\hline HGS vs RP GS & $62(52.7 \%)$ & $18(15.3 \%)$ \\
\hline CGS vs RP GS & $13(11 \%)$ & $1(0.8 \%)$ \\
\hline
\end{tabular}

PGr based on CGS vs HGS and final GS were compared. Bowker test of symmetry was used to compare GS change using the two methods. The GS change was the same using both methods for 41 patients. CGS correlated with RP GS and the HGS was downgraded for 46 patients. CGS was upgraded and HGS correlated with RP GS for 11 patients. CGS was upgraded while the HGS was downgraded for 20 patients. Overall, the CGS is less likely to be downgraded and more likely to be upgraded than the HGS $(p<0.001)$. Conclusions: CGS correlates better with RP GS than HGS when $>2$ grades are present on a 12 core prostate biopsy. CGS has a significantly lower rate of downgrade and predicts the RP GS more accurately than HGS.

878 Is the 2005 International Society of Urological Pathology (ISUP) Recommendation for Gleason Grading Accurate When Three Different Gleason Grade Patterns Are Present in the Same Needle Core?

JA Arias-Stella, SR Williamson, NS Gupta. Henry Ford Health System, Detroit, MI. Background: The 2005 ISUP consensus on Gleason grading recommended that for needle biopsies with patterns 3,4 , and 5 , both the primary pattern and the highest grade should be included in the Gleason score. The rationale is that clinicians use various nomograms to predict pathologic stage or outcome. Since these do not take into account tertiary grade, they may underestimate the prognostic parameters. In this study, we correlate this ISUP recommendation with radical prostatectomy (RP) data. Design: All prostate needle biopsies performed at our institution from 2007 to 2013 were searched for biopsies with patterns 3, 4, and 5 in the same core. Cases were excluded when a core with same grade or higher grade was present in addition to a core with three different grade patterns. Prognostic groups (PGr) were assigned from II to V. RP findings recorded were pathologic stage, Gleason score, tumor volume, and margin and lymph node status. Follow up treatment data were collected

Results: Fifty-one cases showed three Gleason patterns and 41 met our criteria for inclusion. Using ISUP recommendations, 11 cases were graded as $3+5=8$ instead of $3+4=7,29$ cases were graded as $4+5=9$ instead of $4+3=7$, and 1 case was graded as $4+5=9$ instead of $4+4=8$ on the needle biopsies.

Gleason score comparison

\begin{tabular}{|l|l|l|}
\begin{tabular}{|l|l|l|} 
Number of \\
cases
\end{tabular} & $\begin{array}{l}\text { Biopsy Gleason score using ISUP } \\
\text { recommendation }\end{array}$ & $\begin{array}{l}\text { Gleason score without minor pattern 5 } \\
\text { component }\end{array}$ \\
\hline 11 & 358 & 347 \\
\hline 29 & 459 & 437 \\
\hline 1 & 459 & 448 \\
\hline
\end{tabular}

RP was performed in 15 cases. Of these, 14/15 cases were downgraded, 3 from PGr V to PGr II;. 3 from PGr IV to PGr II; 4 from PGr V to PGr III; 1 from PGr IV to PGr III and 3 from PGr V to PGr IV. RP data is provided in the table below.

\begin{tabular}{|c|c|c|c|c|c|}
\hline $\begin{array}{l}\text { RP Gleason } \\
\text { score }\end{array}$ & $\begin{array}{l}\text { RP } \\
\text { number }\end{array}$ & $\begin{array}{l}\text { Margin } \\
\text { positive }\end{array}$ & $\begin{array}{l}\text { Extra prostatic } \\
\text { extension }\end{array}$ & $\begin{array}{l}\text { Seminal vesicle } \\
\text { invasion }\end{array}$ & Lymph node \\
\hline 3475 & 4 & $1 / 4$ & $3 / 4$ & $1 / 4$ & $1 / 4$ \\
\hline 4375 & 7 & $2 / 7$ & $4 / 7$ & $2 / 7$ & $2 / 7$ \\
\hline 4485 & 3 & $0 / 3$ & $2 / 3$ & $0 / 3$ & $2 / 3$ \\
\hline 459 & 1 & $0 / 1$ & $0 / 1$ & $1 / 1$ & $0 / 1$ \\
\hline
\end{tabular}

Conclusions: In our cohort, we found a significant downgrade in Gleason score in the limited number of RP performed in these groups of patients. The ISUP recommendation for including a minor high grade component in the Gleason score overestimated the final RP grade, thereby assigning patients to high grade category (Gr IV, Gr V). As a result, a surgical option was selected or offered less often to patients in these subgroups.

879 Specificity of GATA3 Staining with Special Emphasis on GATA3 and Uroplakin 2 in Urothelial Lesions

$V$ Avadhani, D Buonocore, AASR Mannan, V Ghali, B Wang. St. Luke's - Roosevelt Hospital, New York, NY; Beth Israel Medical Center, New York, NY

Background: GATA3 expression is reported to be sensitive and specific in urothelial and breast carcinomas. Uroplakin II (UPII) is another marker for urothelial differentiation that is reported to be more specific and sensitive than Uroplakin III. Hence, we conducted the study to assess the staining characteristic of UPII and GATA 3 in tissues/tumors of various organs and determine if GATA3 and UPII could be reliably used together to confirm urothelial differentiation.

Design: A total of 84 tumors were studied, including 11 primary squamous cell carcinoma of lung, 10 squamous cell carcinoma of cervix, 20 urothelial carcinoma, 6 paragangliomas, 6 endometrioid adenocarcinoma, 5 papillary carcinoma of kidney, 4 clear cell renal cell carcinoma, 6 prostatic adenocarcinoma, 6 salivary gland tumors $(5$ pleomorphic adenomas and 1 adenoid cystic carcinoma), 5 seminomas and 5 thymomas. Hematoxylin and eosin-stained slides of all cases were reviewed. Immunohistochemical analysis was performed on formalin-fixed, paraffin-embedded 4-mm-thick whole-tissue sections using GATA3 (Biocare, RTU ) and UPII (Biocare, RTU).
Results: Our results are presented in Table1:

Table 1
\begin{tabular}{|l|l|l||}
\hline Tissue type & $\begin{array}{l}\text { lATA3 Positive\% (Positive/ } \\
\text { Total cases) }\end{array}$ & $\begin{array}{l}\text { OPII Positive\% (Positive/Total } \\
\text { cases) }\end{array}$ \\
\hline Urothelial Carcinoma & $100(20 / 20)$ & $85(17 / 20)$ \\
\hline $\begin{array}{l}\text { Squamous cell carcinoma, } \\
\text { lung }\end{array}$ & $36(4 / 11)$ & $0(0 / 11)$ \\
\hline $\begin{array}{l}\text { Squamous cell carcinoma, } \\
\text { cervix }\end{array}$ & $70(7 / 10)$ & $0(0 / 5)$ \\
\hline $\begin{array}{l}\text { Endometrioid } \\
\text { Adenocarcinoma }\end{array}$ & $33(2 / 6)$ & $0(0 / 6)$ \\
\hline Paraganglioma & $100(6 / 6)$ & $0(0 / 6)$ \\
\hline Papillary carcinoma, Renal & $0(0 / 5)$ & $0(0 / 3)$ \\
\hline Clear cell carcinoma, Renal & $0(0 / 4)$ & $0(0 / 4)$ \\
\hline Prostatic adenocarcinoma & $0(0 / 6)$ & $0(0 / 6)$ \\
\hline $\begin{array}{l}\text { Peomorphic adenoma, } \\
\text { Parotid }\end{array}$ & $100(5 / 5)$ & $0(0 / 5)$ \\
\hline \begin{tabular}{lll|} 
Adenoid cystic carcinoma, \\
Parotid
\end{tabular} & $0(0 / 1)$ & $0(0 / 1)$ \\
\hline Seminoma & $0(0 / 5)$ & $0(0 / 5)$ \\
\hline Thymoma & $0(0 / 5)$ & $0(0 / 2)$ \\
\hline Abortus & $100(5 / 5)$ & Not done \\
\hline
\end{tabular}

Uroplakin 2 was positive only in urothelial carcinomas/ tissue with urothelial differentiation, though not diffuse, and with variable intensity(2+-3+). GATA3 staining in urothelial lesions was strong and diffuse. GATA3 was positive in the basal cells of prostate, acinar cells of the parotid, epithelium of distal tubules, normal and metaplastic squamous epithelium of the cervix, epididymal epithelium, cytotrophoblasts, syncytiotrophoblasts of the normal villi and lymphocytes.

Conclusions: Diffuse and strong staining of GATA3 should be paired with UPII to confirm urothelial differentiation. In small biopsies, one has to be extremely careful while reporting GATA3 positivity since it is expressed in benign and malignant tissue of multiple organs as demonstrated in our study.

880 Utility of Immunohistochemical Stains in Differentiating Urothelial Carcinoma from Squamous Cell Carcinoma

$S$ Babayeva, MU Bashir, J Sarungbam, P Khattar, M Zhong. Westchester Medical Center, Valhalla, NY; Harlem Hospital Center, New York, NY.

Background: The histologic distinction between invasive high-grade urothelial carcinoma (UC) and squamous cell carcinoma (SCC) may be difficult. The accurate identification between these entities is critical due to differing prognosis and therapeutic strategies. Here, we want to evaluate and compare new and established Immunohistochemistry (IHC) stains in UC and SCC.

Design: We searched UC and SCC cases from 2010 to 2013 in the system of pathology department at our institution. The pathology reports and H\&E slides were reviewed. The tissue blocks which are suitable for construction of tissue microarray (TMA) were selected. The TMA were build from 142 cases, including: 93 UC of both upper and lower urinary track, 48 cases of SCC from lung, head and neck, gyn and other sites. The TMA slides were subjected to IHC staining of CK5/6, P63, P40 and GATA3.

Results: Ck5/6, p63, p40 and GATA3 antibodies demonstrated positive staining in pure squamous cell carcinoma in $45(93.8 \%), 45(93.8 \%), 44(91.7 \%)$ and $12(25 \%)$ cases, respectively. Urothelial carcinomas showed reactivity for ck5/6, p63, p40 and GATA3 for $66(71.0 \%), 88(94.6 \%), 85(91.4 \%)$ and $86(92.4 \%)$ cases, respectively. Although some SCC can be GATA3 positive, unlike in UC, staining is more focal and weaker. Conclusions: 1 . P40 and P63 showed similar positivity in both UC and SCC.

2. GATA3 demonstrated the highest specificity among all markers to differentiate UC from SCC. It may be a useful maker when diagnosing metastatic UC to the lung. 3. Combination of GATA3 and p63 has high sensitivity for UC.

881 Novel Technique of Sampling the Urinary Bladder in Radical Cystectomy Specimens for Urothelial Carcinoma

CG Ball, J Swift, TA Flood, EC Belanger, KT Mai. Eastern Ontario Research Laboratory Association / University of Ottawa, Ottawa, ON, Canada.

Background: Sampling of the urinary bladder in radical cystectomy specimens is usually performed by obtaining three or more full thickness strips of bladder wall, in sagittal planes, extending from the bladder neck to the dome. While pathologic assessment of specimens is often sufficient for continuing clinical follow-up and therapy, it is hindered by the difficulty in identifying the anatomical relationship of the urothelial carcinoma to the remaining urinary bladder.

Design: Fifty radical cystectomy specimens (39 from men, 11 from women) were identified; these comprised 9 cases of superficial urothelial carcinoma, and 41 of muscle invasive urothelial carcinoma. Each of these was bisected in the transverse plane at the middle portion of the urinary bladder; these were then fixed without stretching in $10 \%$ buffered formalin for at least 24 hours. After fixation, the urinary bladders (while still attached to the prostate or uterus) were serially sectioned in the transverse planes, from the uinary bladder neck to the dome, in $5 \mathrm{~mm}$ thick rings. These sections were arranged in order, mapped, and photographed (see figure). At least one ring of tissue was submitted in toto, along with other interesting or representative sections. 


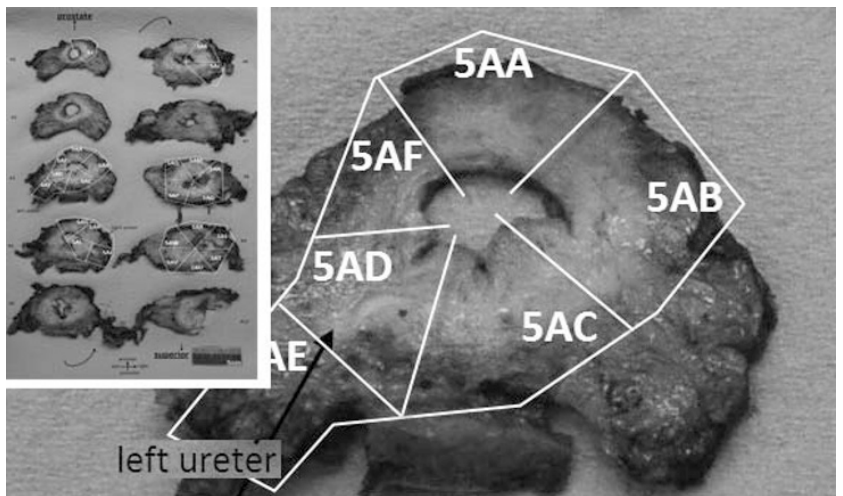

Results: Our proposed method of transverse sections results in no real increase in the number of sections submitted for microscopic examination, with $12 \pm 5$ sections for the transverse sections method versus $11 \pm 5$ for the "tissue strip" method. The advantages of our method are: a) consistency and ease of sampling; b) suitability for determining depth of invasion by gross examination; c) improved identification of tumor size(s), topographic location, and multifocality; d) suitability for mapping of neoplastic lesions; e) suitability for re-examination for further sampling; and e) increased tissue sampling that exceeds $50 \%$ of urinary bladder mucosa.

Conclusions: Transverse sectioning of the urinary bladder facilitates and maximizes the pathological examination of the bladder wall in radical cystectomy specimens.

882 Basal-Like Variant of Non-Invasive Urothelial Carcinoma: A Variant of Urothelial Carcinoma with Immunohistochemical Features of Basal-Like Urothelial Cells Associated with High Rate of Recurrence and Invasion

CG Ball, TA Flood, EC Belanger, KT Mai. Eastern Ontario Regional Laboratory Association / University of Ottawa, Ottawa, ON, Canada.

Background: Low grade urothelial carcinoma frequently displays positive CD44 reactivity for urothelial stem cells in the basal and suprabasal layers of neoplastic urothelium. We investigated the clinical and pathological significance of a subgroup of non-invasive urothelial carcinoma that displays full thickness reactivity for CD44. Design: A series of 123 consecutive cases of non-invasive urothelial carcinoma without squamous differentiation was reviewed; these were immunostained with the basal cell markers CD44 CK5, CK34bE12, bc12, EP4, and p63. Those cases selected for study showed strongly positive CK 5 immunoreactivity in more than $1 / 2$ thickness of neoplastic urothelium and more than $1 / 2$ the area of non-invasive tumor. We subsequently designated this subgroup as basal-like urothelial carcinoma (BUC). Ten cases of lowgrade and high-grade non-BUC were selected as controls.

Results: There were 20 cases of non-invasive low-grade papillary BUC, and 14 of high-grade BUC; cases with strong CD44 but limited CK5 reactivity were excluded. Immunoreactivity to CK5 is stronger than that of CD44, which tends to be uniform and expansive. Cytokeratin 34bE12 was more sensitive but non specific; whereas p63, bcl2, and EP4 were neither sensitive nor specific in detecting BUC. Mild to moderate nuclear atypia was seen to be characteristic of BUC. Focal squamous and/or glandular differentiation were occasionally seen. Low-grade BUC was associated with multifocal disease, developing up to 10 concomitant tumors. All cases with at least 12 months' follow-up were associated with recurrence, up to 4 times per year. Progression of low-grade BUC to high-grade disease, or to development of superficial invasive carcinoma, within a 3 to 42 month period, was seen in 6 and 19 cases, respectively. Distant metastasis occurred in 5 cases during a 36 month period following diagnosis of low-grade or high-grade non-invasive BUC.

Conclusions: In analogy to basaloid carcinoma in other sites of the body, basal-like urothelial carcinoma likely represents the basal-like counterpart of common urothelial carcinomas. Identification of non-invasive basal-like urothelial carcinoma with CD44/ CK5 immunoreactivity involving full or nearly full thickness is helpful to identify a subgroup of aggressive non-invasive urothelial carcinomas.

883 "Early" and Other Patterns Associated with Intraductal Carcinoma of the Prostate (IDC-P): A Study Focusing on Proximal Duct (PD) Involvement and PTEN Loss

BS Banach, GP Paner. University of Chicago, Chicago, IL.

Background: The morphologic overlap between cribriform high grade prostatic intraepithelial neoplasia (HGPIN) and IDC-P is well recognized. Criteria were described (Guo and Epstein; Cohen et al.) to distinguish IDC-P from HGPIN. However, it is unclear if IDC-P can have morphology similar to the non-cribriform HGPIN or can have other patterns exclusive of the existing diagnostic criteria.

Design: A representative section from 98 radical prostatectomies (mostly $>$ GS7) that showed the prostate promontory were examined. All sections were immunostained for PIN4 and PTEN. PDs were identified as elongated ducts that converged towards the prostate promontory/prostatic urethra. IDC-P identified based on existing criteria were labeled as "classic" IDC-P. Intraductal spread of PCa in PDs was accepted if all the following criteria were met: 1) neoplasia present within PDs, 2) non-invasiveness confirmed by PIN4, 3) neoplasia in ducts contiguous with classic IDC-P in PDs or peripheral ducts/acini, and 4) loss of PTEN. PCa involvement of ejaculatory duct or prostatic utricle was discounted. Morphologic patterns for all cases that met the above criteria were documented.
Results: Of 98 cases, 25 met the criteria for intraductal spread of PCa in PDs. Nonclassic patterns of IDC-P were identified including partial duct involvement (18/25, $72 \%)$ and overriding of duct urothelium $(4 / 25,16 \%)$. Also, associated with IDC-P were flat/tufted $(14 / 25,56 \%)$, micropapillary $(4 / 25,16 \%)$, and inverted $(1 / 25,4 \%)$ patterns. These flat/tufted, micropapillary and inverted patterns were in non-expanded ducts without large (X6) nuclei and are similar to those seen in HGPIN. As per criteria, all these patterns were contiguous with classic IDC-P and exhibited PTEN loss. Overall, IDC-P was seen in 62/98 (63\%) of cases.

Conclusions: Non-classic patterns of IDC-P are identified within the proximal PDs. Our findings also suggest that IDC-P is associated with pattens that resemble non-cribriform HGPIN. Such patterns may represent the "advancing edge" of IDC-P and whether to label these as IDC-P needs further study.

884 Identification and Validation of Protein Biomarkers of Response to Neoadjuvant Chemotherapy in Muscle Invasive Urothelial Carcinoma A Baras, $N$ Gandi, E Munari, SF Faraj, M Hoque, M Schoenberg, T Bivalacqua, GJ Netto. Johns Hopkins University, Baltimore, MD.

Background: Neoadjuvant chemotherapy (neoadj-Rx) prior to cystectomy results in $5-10 \%$ benefit in 5-year survival rate in muscle invasive bladder cancer (MIBC) patients. More importantlyly, those who achieve a complete response (ypT0) have a 5 year survival rate of $\sim 80 \%$ as opposed to $\sim 40 \%$ for those who do not. However, neoadj-Rx is not widely adopted due to concerns related to delay of cystectomy, potential sideeffects and inability to predict effectiveness. Recently suggested "molecular signatures" of chemoresponsiveness are yet to be translated into clinical practice.

Design: mRNA expression data from prior report on neoadj-Rx treated MIBC cohort (Kato et. al) were analyzed in combination with antibody database of the Human Protein Atlas (HPA). We thus identified classifier candidate biomarkers that can be detectable by immunohistochemistry (IHC) in TURB biopsy material prior to neoadj-Rx. The candidate biomarkers were subsequently validated in an independent cohort of 23 MIBC, treated with neoadj-Rx in our hospital, whose TURB specimens were used to construct a tissue microarray.

Results: By filtering based on large amplitude mRNA changes and the area under the receiver operator curve (AUROC), we identified 21 genes whose mRNA expression profiles differentiate response to neoadj-Rx $(n=33)$, Figure 1A. Using the HPA, we found that 8 of these genes had commercially available antibodies that exhibited differential staining across a set of urothelial carcinomas cataloged in the HPA. In addition 12 normal tissues were identified from the HPA that can serve as positive and negative controls for the 8 proteins. A combination of 2 of these markers in a multivariate logistic regression model have yielded excellent overall performance (AUROC of 0.92) in identifying patients in our independent validation cohort that are not likely to benefit from neoadjuvant Rx due to chemoresistance, Figure 1B \& 1C.
A

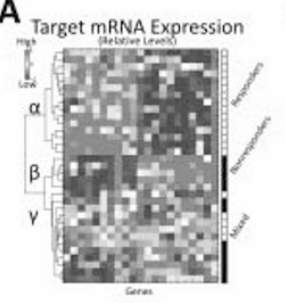

B
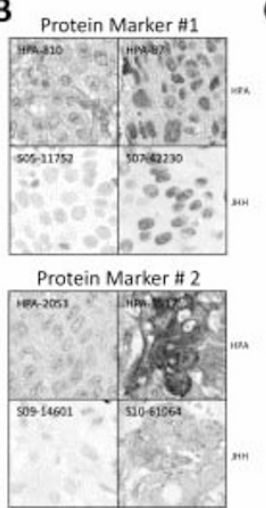

C

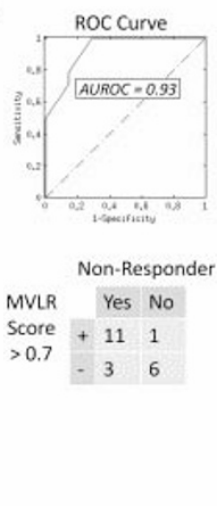

Figure 1. (A) Heatmap and hierarchical dendrogram (rows - samples, columns genes) using the 21 target mRNA species identified from the Kato et al. cohort showed robust differences that correlate well to responder status. Additionally, unsupervised clustering revealed three distinct yet related sample groups designated as $\alpha, \beta$, and $y$ in the dendrogram, which correlated to response status. (B) Representative positive and negative cases by IHC for 2 of the putative protein biomarkers from both the Human Protein Atlas (HPA) and the Johns Hopkins Hospital (JHH). (C) The combination of two protein biomarkers into a multivariate logistic regression (MVLR) model yielded a favorable AUROC of 0.93 . Using a cutoff of MVLR score $>0.7$, these two protein biomarkers achieved an excellent positive predictive value (PPV) of 0.92 . Cases with a MVLR score $<0.7$ exhibited a neoadj-Rx response rate of $66 \%$, which is roughly $2 x$ that of neoadj-Rx treated MIBC as a whole.

Conclusions: We illustrate the feasibilty of translating gene expression signature data on neoadj-Rx response into IHC classifier applicable to TURB specimen. The excellent perfomance achieved by the initial two markers in our study is being further assessed in a larger cohort. 
$885 \quad$ Handling and Reporting of Orchiectomy Specimens with Testicular Cancer: Areas of Consensus and Variation among 25 Experts and 225 European Pathologists

D Berney, F Algaba, M Amin, P Camparo, E Comperat, A Lopez-Beltran, R Montironi, $M$ Varma, L Egevad. Fundacio Puigvert-University Autonomous, Barcelona, Spain; Centre de Pathologie Amiens, Paris, France; Queen Mary University of London, London, United Kingdom; University Hospital of Wales, Cardiff, United Kingdom; Hopital La Pitié-Salpetrière, Paris, France; Cordoba University Medical School, Cordoba, Spain; Polytechnic University of the Marche Region, Ancona, Italy; Karolinska Institutet, Stockholm, Stockholm, Sweden; Cedars-Sinai Medical Center, Los Angeles, CA.

Background: Testicular pathology is challenging due to the rarity and complexity of neoplasms encountered. We investigated the variation in handling and reporting of these tumors.

Design: A survey was developed by the European Network of Uro-Pathology (ENUP) and sent to all ENUP members as well as selected experts.

Results: Replies were received from 25 experts (E) and 225 ENUP members. Both groups had remarkably similar responses. For macroscopy, there was a greater than $90 \%$ consensus that the cord margin should be sampled. 2 or more other cord blocks were taken by $48 \%$ (E) and $56 \%$ (ENUP) and were taken before incising the testis by $84 \%$ (E) and $70 \%$ (ENUP). The rete testis was sampled by $100 \%$ (E) and $98 \%$ (ENUP). For microscopy, WHO 2004 classification was used by $100 \%$ (E) and $99 \%$ (ENUP). Area of disagreement included immaturity in teratomas, reported by $32 \%$ (E) but $68 \%$ (ENUP). Type of tumor involved in vascular invasion was reported by $56 \%$ (E) and $54 \%$ (ENUP); lymphatic and vascular invasion were distinguished by $28 \%$ (E) and $41 \%$ (ENUP). Although the presence of rete invasion was reported by $96 \%$ (E) and $94 \%$ (ENUP), the distinction between pagetoid and stromal invasion was made by $96 \%$ (E) but only $63 \%$ (ENUP). Immunohistochemistry for 14 different markers was variably used in more than $50 \%$ of cases by $68 \%$ (ENUP) and $12 \%$ (E). Staging revealed the greatest areas of disagreement. Invasion of the inner serosal lining of the testis without vascular invasion was interpreted as T1 by $52 \%$ (E) and $67 \%$ (ENUP), but T2 by the remainder. Tumor invading the hilar adipose tissue adjacent to the epididymis without vascular invasion was interpreted as T1: $40 \%$ (E), 43\% (ENUP), T2: 36\% (E), 30\% (ENUP) and T3: 24\% (E), 27\% (ENUP).

Conclusions: There is remarkable consensus in reporting and handling in many areas of testicular pathology. Significant areas of disagreement included select aspects of staging and reporting of histologic types, both of which have the potential to impact on therapy. Further focused study and/or development of consensus will help standardize the reporting of these tumors internationally.

\section{6} Origin

SM Bezerra, SF Faraj, S Karram, MP Schoenberg, TJ Bivalacqua, TL Lotan, GJ Netto. Johns Hopkins University, Baltimore, MD.

Background: GATA3 is a transcription factor that plays an important role in early T-cell and mammary gland development. Recently, GATA3 has been shown to be a sensitive marker for urothelial carcinoma as well as some of its variants. GATA3 expression has yet to be assesed in small cell carcinoma of urinary bladder. In the current study we evaluate GATA3 expression in small cell carcinoma of baldder and assess its utility in the differential diagnosis with small cell carcinoma of lung primary.

Design: Formalin-fixed paraffin-embedded tissues from 28 small cell carcinomas including 12 cases of small cell carcinoma of the bladder and 16 cases of small cell carcinoma of the lung were retrieved from our surgical pathology archives and used to build a tissue microarray. Paired tumor and non-neoplastic bladder and/or lung were spotted 3 times each. GATA3 nuclear expression was evaluated using standard immunohistochemistry (GATA3: clone L50-823, BioCare Medical, CA). Intensity (weak, moderate and strong) and extent (percentage) of expression were assessed in each spot. For each tumor, mean extent of expression was calculated and the highest intensity of expression was recorded. Extent positivity was further categorized as: Focal $(1-25 \%)$, multifocal $(>25 \%)$ and diffuse $(>75 \%)$.

Results: Nuclear GATA3 expression was encountered in $21 \%(6 / 28)$ of all studied cases including 4 small cell carcinomas of the bladder (4/12 - 33\%) and 2 small cell carcinomas of the lung $(2 / 16-12.5 \%)$. Among bladder tumors, strong and diffuse $(>75 \%)$ GATA3 labeling was seen in 1 case $(1 / 12-8 \%)$; weak and focal positivity was observed in the 3 additional cases (3/12-25\%). Among lung small cell carcinomas, both cases $(2 / 16-12.5 \%)$ revealed weak and focal nuclear positivity.

Conclusions: We found GATA 3 to be positive in one third of small cell carcinomas of bladder. However, GATA3 expression is not entirely specific for small cell carcinomas of bladder origin given its positivity in a minority of lung small cell carcinomas. The findings should be taken into consideration when assessing primary site of origin of metastatic small cell carcinomas.

887 Human Papillomavirus Infection and Immunohistochemical p16 Expression in Penile Squamous Cell Carcinomas

SM Bezerra, M Ball, N Gonzalez-Roibon, SF Faraj, A Chaux, E Munari, A Burnett, GJ Netto. Johns Hopkins Medical Institutions, Baltimore, MD; Norte University, Asunción, Paraguay.

Background: Approximately $50 \%$ of penile carcinomas are associated with highrisk human papillomavirus (HR-HPV) infection. The gold standard for human papillomavirus (HPV) detection is the polymerase chain reaction (PCR) assay. In the current study, we evaluate the correlation of immunohistochemistry for $\mathrm{p} 16^{\text {INK4a }}$ expression, and in situ hybridization (ISH) for HR-HPV detection with clinicopathologic features of penile carcinoma and outcome in a cohort of North American patients.

Design: Two tissue microarrays were constructed from 53 invasive penile squamous cell carcinoma at Johns Hopkins Hospital (1985 to 2013). Immunohistochemistry for p16 ${ }^{\text {INK4a }}$ (CINtec Kit) was performed and each case was classified as negative p16 $6^{\text {INK4a }}$ expression (absence of $16^{\mathrm{INK} 4 \mathrm{a}}$ staining in all tumor cells or positivity in some, but not all, tumor cells) or positive $\mathrm{p} 16^{\mathrm{INK} 4 \mathrm{a}}$ expression ( $\mathrm{p} 16^{\mathrm{INK} 4 \mathrm{a}}$ positivity in all tumor cells). In addition, HR-HPV ISH was performed (INFORM HPV III family 16 probe (B) cocktail). A case was considered positive when a punctuated blue stain was observed in nuclei of tumor cells.

Results: HPV was detected in 8 cases (15\%), showing a significant association with histologic subtype $(\mathrm{P}=0.001)$, histologic grade $(\mathrm{P}=0.03)$ and lymphovascular invasion $(\mathrm{P}=0.001)$ but not with perineural invasion $(\mathrm{P}=0.41)$. Overexpression of $\mathrm{p} 16^{\mathrm{INK} 4 \mathrm{a}}$ was found in 23 cases $(44 \%)$, showing a significant association with histologic subtype $(\mathrm{P}=0.006)$, histologic grade $(\mathrm{P}=0.005)$, and lymphovascular invasion $(\mathrm{P}=0.002)$ but not with perineural invasion $(\mathrm{P}=0.39)$. The association between HPV infection and p16 $6^{\text {INK4a }}$ overexpression was significant $(\mathrm{P}=0.001)$. Regarding outcome analyses, neither HR-HPV status nor $\mathrm{p} 16^{\mathrm{INK} 4 \mathrm{a}}$ overexpression significantly predicted overall survival $(\mathrm{P}=0.72$ and $\mathrm{P}=0.89$, respectively). Similar results were found for cancer-specific survival regarding HR-HPV status $(\mathrm{P}=0.59)$ and $\mathrm{p} 16^{\mathrm{INK} 4 \mathrm{a}}$ overexpression $(\mathrm{P}=0.24)$

Conclusions: HR-HPV status by ISH and $\mathrm{p} 16^{\mathrm{INK} 4 \mathrm{a}}$ overexpression were significantly associated with histologic subtype, histologic grade, and lymphovascular invasion in penile cancer. Nevertheless, neither predicted overall survival or cancer-specific survival, suggesting their limited value as prognosticators of outcome in our cohort of North American patients.

\section{8} GATA3 Expression in MiTF/TFE Family TranslocationAssociated Renal Carcinomas

SM Bezerra, SF Faraj, E Munari, N Gonzalez-Roibon, G Martignoni, M Allaf, P Argani, GJ Netto. Johns Hopkins University, Baltimore, MD; Universitá di Verona, Verona, Italy. Background: GATA3 is a transcription factor involved in the development of several organs, including mammary gland, skin and kidney and has been suggested as a marker of urothelial carcinoma of bladder as well as upper urinary tract. Recently we found GATA3 to be expressed in a minority of oncocytomas and collecting duct carcinoma of kidney. In the current study we evaluate the expression of GATA3 in a set of $38 \mathrm{MiTF} /$ TFE family Translocation-associated renal carcinomas.

Design: Formalin-fixed paraffin-embedded tissues from 21 TFE3 (Xp11.2) renal carcinomas and 17 TFEB $[\mathrm{t}(6 ; 11)]$ renal carcinomas were retrieved from our surgical pathology archives and the consultation service of one author and used to build 2 tissue microarrays. Paired tumor and non-neoplastic kidney were spotted at least 4 times each. GATA3 nuclear expression was evaluated using standard immunohistochemistry (GATA3: clone L50-823, BioCare Medical, CA). Intensity (weak, moderate and strong) and extent (percentage) of expression were assessed in each spot. For each tumor, mean extent of expression was calculated and the highest intensity of expression was recorded. Results: Overall, we found positive GATA3 expression in 6/38 (16\%) cases of MiTF/ TFE Translocation-associated renal carcinomas. Among TFE3 renal carcinomas, GATA3 was positive in $2 / 21(10 \%)$ of cases $(5 \%, 1+$ intensity and $100 \%, 3+$ intensity, respectively). Four of 17 (23\%) TFEB renal carcinomas were positive for GATA3 (5\%, $1+$ intensity ; 39\%,3+ intensity; $40 \%, 1+$ intensity and 47\%, 2+ intensity, respectively). Conclusions: We found GATA3 expression in a minority of MiTF/TFE Translocationassociated renal carcinomas. TFEB tumors had a higher likelihood of GATA3 expression compared to TFE 3 tumors. The findings should be taken into consideration when using this marker in the differential diagnosis of primary or metastatic renal tumors (urothelial carcinoma Vs renal cell carcinoma).

889 Prostate Carcinoma

$S$ Bhele, Z Ma, S Mohanty, S Salman, MB Amin, B Balzer, BS Knudsen, A Gertych. Cedars-Sinai Medical Center, Los Angeles, CA; University of Southern California, Los Angeles, CA

Background: Accurate assessment of the composition of the Gleason score in prostate cancer is critical for patient management. Image analysis could aid in consistent, reproducible and objective quantification of Gleason patterns $(\mathrm{G})$. Existing computerassisted analytical approaches employ segmentation and histomorphometry of glands and often evaluate large image regions without resolving tumor grades. To date, none possesses sufficiently high discriminatory power to quantify G3 and G4. We propose to employ high-level image processing toolkits, recently developed for face recognition, to capture the architecture of single glands and distinguish G4 from benign epithelium (BE), G3 and stroma (ST).

Design: One slide each was selected from 38 cases of radical prostatectomy (RP). Slides were digitized by a high-resolution scanner (Leica SCN400F) and images of 1200x1200 pixels were used for analysis. Two pathologists identified and manually circled BE, G3, G4 and ST in 105 images. Quantitative features of intensity and texture were extracted from a training set of 25 images to develop a supervised classifier. 80 images were used to evaluate the performance of the classifier to distinguish cancer grades, BE and ST. The concordance between computed and human annotation was assessed by calculating the percentage overlap.

Results: Our algorithm distinguished four tissue components, and consistently classified benign, G3 and G4 in mixed images. The concordance of the algorithm with human outlines in G3, G4 and BE was $67-81 \%$ with a coefficient of variation of $5-10 \%$. The overall performance increased in images with a single Gleason grade.

\begin{tabular}{|c|c|c|c|c|c|c|c|c|c|}
\hline Tissue components & \multicolumn{3}{|c|}{ G3-G4-ST } & \multicolumn{3}{|c|}{ G3-BE-ST } & \multicolumn{3}{c|}{ G4-BE-ST } \\
\hline Tissue & G3 & G4 & ST & G3 & BE & ST & G4 & BE & ST \\
\hline Concordance [\%] & $67 \%$ & $71 \%$ & $73 \%$ & $69 \%$ & $76 \%$ & $77 \%$ & $73 \%$ & $81 \%$ & $82 \%$ \\
\hline
\end{tabular}


Conclusions: This automated method has potential utility to objectively provide the percentage of G3 and G4 and differentiate G3+4 from G4+3. While the extent of G4 is associated with the risk of cancer recurrence after RP, the amount of G3 in biopsies is used to enroll patients in active surveillance programs. In both circumstances our image analysis approach carries a promise to improve patient care.

890 Tumor Extent Evaluation in Surgical Specimens of Patients Submitted to Radical Prostatectomy: A Comparative Study

A Billis, LLL Freitas, L Meirelles, PAM Odorissi, CM de Angelis, KR Carvalho, LC Fernandes. School of Medical Sciences, State University of Campinas (Unicamp), Campinas, SP, Brazil.

Background: At the International Society of Urological Pathology (ISUP) consensus conference on handling and staging radical prostatectomy specimens, a recommendation was made that some quantitative estimate of cancer volume should be performed, of which the nature depends on routine practice at the pathology laboratory. The most accurate method is to use computer assisted image analysis systems, which is not feasible for routine clinical practice. In step sectioned specimens, we compared a time consuming semiquantitative point count method vs evaluation of number of involved quadrants (blocks).

Design: Prostates from 422 patients submitted to radical prostatectomy (RP) were step sectioned at 3 to $5 \mathrm{~mm}$ intervals. Each transverse section of the prostate was subdivided into 2 anterolateral and 2 posterolateral quadrants. Each quadrant was embedded in one paraffin block. Semiquantitative point count evaluation of tumor extent in each quadrant (block) was performed according to a method previously described. Time to biochemical recurrence (TBCR) after RP was analyzed with the Kaplan-Meier product-limit analysis using the log-rank test and prediction of TBCR using univariate and multivariate Cox proportional hazards model.

Results: Using the semiquantitative method, the mean and median positive points were 36 and 27 (range 1-225); the number of involved quadrants (blocks) was 14 and 13 (range 1-41). Comparing the 2 methods of tumor extent evaluation, there was no significant difference associated with age and race. More extensive tumors in both methods were significantly associated with higher preoperative PSA, more patients with clinical stage $\mathrm{T} 2$, more specimens showing pathological stage $\mathrm{T} 3 \mathrm{a} / \mathrm{T} 3 \mathrm{~b}$ and positive surgical margins, and higher biopsy and pathological Gleason score. More extensive tumors in both methods showed shorter TBCR in Kaplan-Meier curve: log-rank, $p=0.034$ using the semiquantitative method; and, log-rank, $p=0.032$ using the number of involved blocks. Both methods significantly predicted shorter TBCR in Cox univariate analysis: $\mathrm{p}=0.036$ using the semiquantitative method; and, $\mathrm{p}=0.034$ using the number of positive blocks. However, both were not independent predictors in Cox multivariate analysis in a model including positive surgical margins, preoperative PSA, pathologic stage and Gleason score.

Conclusions: In step sectioned surgical specimens, the number of involved quadrants (paraffin blocks) for tumor extent evaluation is equivalent to a time consuming semiquantitative point count method.

891 Topography of Index Tumor in Surgical Specimens of Patients Submitted to Radical Prostatectomy: Clinicopathological Associations

A Billis, LLL Freitas, L Meirelles, MVB Mota, FFC Barra, JPU Fontenele, LGF Cortes, AA Tavares, U Ferreira. School of Medical Sciences, State University of Campinas (Unicamp), Campinas, SP, Brazil.

Background: In a previous study, we found that total and index tumor extent in radical prostatectomies (RP) significantly predicted shorter time to biochemical recurrence (TBCR) after surgery on univariate analysis but only index tumor extent was an independent predictor on multivariate analysis. In this study, we analyzed clinicopathological associations according to predominant topography of index tumor: transition zone (TZ) vs peripheral zone (PZ), and basal (BT) vs apical (AT) topography. Design: Prostate surgical specimens were step sectioned from 441 patients submitted to RP. Each transverse section of the prostate was subdivided into 2 anterolateral and 2 posterolateral quadrants. Tumor extent at RP was evaluated and mapped using a semiquantitative point count method. TBCR after RP was analyzed with the KaplanMeier curves and prediction of TBCR using univariate and multivariate Cox model. Results: Considering index tumors in $\mathrm{TZ}$ vs $\mathrm{PZ}$, there was no significant difference associated with age, preoperative PSA, race, clinical stage, pathological stage, positive surgical margins (PSM), bladder neck involvement (BNI) or apical involvement (AI) with or without PSM, extraprostatic extension (EPE), seminal vesicle invasion (SVI), TBCR in Kaplan-Meier curve ( $\mathrm{p}=0.483)$, and prediction of shorter TBCR in Cox univariate analysis $(\mathrm{p}=0.486)$. TZ tumors, showed significantly lower biopsy and pathologic Gleason score. Considering index tumors in basal vs apical topography, there was no significant difference associated with age, preoperative PSA, biopsy and pathologic Gleason score, race, and clinical stage. Index tumors in BT, showed a significantly higher pathologic stage, BNI, BNI with PSM, EPE, and SVI; and, index tumors in AT, showed higher AI and AI with PSM. BT tumors showed significantly shorter TBCR in Kaplan-Meier curve $(\mathrm{p}=0.003)$, significant prediction of shorter TBCR in Cox univariate analysis $(\mathrm{p}=0.004)$ and were independent predictors of TBCR in multivariate analysis $(\mathrm{p}=0.013)$ in a model not including SVI.

Conclusions: TZ index tumors (vs PZ) showed lower biopsy and pathologic Gleason score and no other significant differences. Index tumors in basal topography (vs apical) are associated with several adverse clinicopathological findings including shorter TBCR in Kaplan-Meier curves, prediction of shorter TBCR on Cox univariate analysis, and are independent predictors on multivariate analysis in a model not including SVI.
$892 \quad$ Clinicopathologic Findings Associated with Insignificant Prostate Cancer in Surgical Specimens of Patients Submitted to Radical Prostatectomy

A Billis, MM Quintal, LLL Freitas, L Meirelles, MVB Mota, FFC Barra, JPU Fontenele, LGF Cortes, AA Tavares, LA Magna. School of Medical Sciences, State University of Campinas (Unicamp), Campinas, SP, Brazil.

Background: Patients harboring insignificant cancer ( $\mathrm{InsCa}$ ) in surgical specimens of radical prostatectomy (RP) have almost $100 \%$ cancer-specific survival after surgery. Our aim was to associate InsCa with several clinicopathological findings. We defined InsCa in RP, patients with T1c clinical stage, organ confined tumor (pT2), limited tumor extent, negative surgical margins, and Gleason score $\leq 6$.

Design: From a total of 401 consecutive patients submitted to RP, $21(5.2 \%)$ patients met criteria for InsCa. The mean follow-up after surgery was 78 months (median 75 , range 32-148). No patient during this period had biochemical recurrence considered as PSA $0.2 \mathrm{ng} / \mathrm{mL}$ or greater according to the AUA recommendation. Tumor extent was evaluated by a previously described semiquantitative point count method. A total of $\leq 10$ positive points (limited tumor extent) correspond roughly to a $\leq 0.5 \mathrm{~cm}^{3}$ tumor. Results: The mean age was 61 years (median 70, range 19-136), mean preoperative PSA $7.62 \mathrm{ng} / \mathrm{mL}$ (median 7.3, range 4.4-12.30), mean pathologic PSA density (ng/mL/ prostate weight) 0.18 (median 0.16 , range $0.11-0.27$ ), mean prostate weight $46 \mathrm{~g}$ (median 49 , range $21-75$ ), all patients with biopsy Gleason score $3+3=6$, mean number of biopsy cores with carcinoma 1.5 (median 1 , range 1-3), mean maximum length of cancer in a single core $1.3 \mathrm{~mm}$ (median 1.25 , range $0.5-2.50$ ), mean maximum percentage of cancer in a single core $16.3 \%$ (median 13.1, range $4.5-50$ ), mean length of carcinoma in all cores of the biopsy $1.6 \mathrm{~mm}$ (mean 1.5 , range $0.5-3$ ), mean percentage length of carcinoma in all cores $6.2 \%$ (mean 3 , range $0.4-50$ ), and mean number of cores examined 9.5 (median 8, range 2-20). Comparing Epstein's criteria on needle biopsy predictive of InsCa, our findings were concordant in $100 \%, 42.86 \%, 100 \%, 95.24 \%$, and $100 \%$ patients for stage T1c, PSA density $\leq 0.15$, no Gleason pattern 4 or $5,<3$ cores with cancer, and no core with $>50 \%$ cancer, respectively.

Conclusions: No patient with InsCa cancer in surgical specimens had biochemical recurrence in a mean follow-up after surgery of 78 months (median 75 , range 32-148). The clinicopathological findings were in accordance with Epstein's criteria on needle biopsy predictive of InsCa, except for PSA density. We found a wider range of PSA density $(0.11-0.27)$ associated with InsCa. It seems that the criterion for a cut-off $\leq$ 0.15 for PSA density may be higher.

893 A Subset of Prostatic Basal Cell Carcinomas Harbor the MYB Rearrangements That Characterize Salivary Gland Adenoid Cystic Carcinoma

JA Bishop, R Yonescu, D Batista, JI Epstein, WH Westra. The Johns Hopkins Medical Institutions, Baltimore, MD.

Background: Adenoid cystic carcinoma (ACC) is a basaloid tumor consisting of myoepithelial and ductal cells that arise from major and minor salivary glands, but can also occur in non-salivary sites including the skin, breast and vulva. A rare form of prostate carcinoma exhibits adenoid cystic-like features, but these are generally not regarded as true ACCs largely based on the absence of prostatic myoepithelial cells. Instead, they are now classified as "basal cell carcinoma" and are believed to occupy a position in the spectrum of basaloid proliferations of the prostate ranging from basal cell hyperplasia-like carcinomas to solid basaloid carcinomas. True ACCs of salivary and non-salivary origin often harbor $M Y B$ translocations resulting in the MYB-NFIB fusion protein. $M Y B$ analysis could help refine the classification of prostatic basaloid carcinomas and establish the true nature of those that exhibit adenoid cystic features. Design: Paraffin embedded tissue from 10 prostatic BCCs was retrieved from the consult files of one of the authors. All cases exhibited prominent basaloid histology, and 6 of them were histologically indistinguishable from salivary ACC. Break-apart fluorescence in situ hybridization (FISH) for $M Y B$ was performed on each case.

Results: Overall, 2 of $10(20 \%)$ prostatic BCCs were positive for the MYB rearrangement. The positive cases were restricted to the adenoid cystic-like BCCs (2 of $6,33 \%$ ). The MYB rearrangement was not identified in any of the BCCs that lacked ACC-like features (0 of 4$)$.

Conclusions: A subset of prostatic BCCs possesses the $M Y B$ rearrangement that characterizes ACC in other sites. Prostate BCC may consist of two distinct tumor types: "true" ACCs that harbor MYB rearrangements at a rate similar to ACC of salivary glands, and basaloid carcinomas that are translocation-negative.

894 SPOP Mutations in Prostate Cancer across Ethnically and Geographically Diverse Patient Cohorts

$M$ Blattner, D Lee, K Park, F Khani, S Ramazanoglu, A Sboner, F Demichelis, JM Mosquera, B Robinson, C Barbieri, M Rubin. Weill Cornell Medical College, NY, NY; University of Trento, Trento, Italy; Institute for Precision Medicine, Weill Cornell Medical College/NYPH, NY, NY.

Background: Recurrent mutations in the Speckle-Type POZ Protein (SPOP) gene have been reported in up to $15 \%$ of prostate cancers. However, the frequency and features of cancers with these mutations across different populations is unknown. In this study, we sought to investigate $S P O P$ mutations across diverse cohorts and validate a series of assays for mutational analysis of formalin-fixed paraffin-embedded material.

Design: 720 prostate cancer samples from six different international cohorts spanning Caucasian, African American, and Asian patients, including both PSA-screened and unscreened populations, were screened for their SPOP mutation status. An assay employing High Resolution Melting Analysis and Sanger sequencing was optimized 
to screen for somatic mutations in recurrently altered areas of the $S P O P$ gene. Status of $S P O P$ was correlated to molecular features (ERG rearrangement, PTEN deletion, $C H D 1$ deletion) as well as clinical and pathologic features.

Results: The overall frequency of SPOP mutations was $8.1 \%$ in 720 patient's samples, ranging from $4.6 \%$ in the African American cohort to $14.4 \%$ in the non-African American cohort. The frequency of SPOP mutations in prostate cancers from the Asian cohort was $6.9 \%$. There were no significant differences in frequency between ethnicities or cohorts $(\mathrm{p}=0.14) . S P O P$ mutation was inversely associated with $E R G$ rearrangement $(\mathrm{p}<0.01)$, and $S P O P$ mutant cancers had higher rates of $C H D 1$ deletions $(\mathrm{p}<0.01)$. There were no significant differences in rates or time to biochemical recurrence in $S P O P$ wild-type vs mutants ( $\mathrm{p}=0.18,0.30$ respectively). The mutational assays showed excellent sensitivity and specificity in high quality samples.

Conclusions: $S P O P$ is mutated in $4.6-14.4 \%$ of prostate cancer patients with different ethnic and demographic backgrounds. Mutually exclusivity of SPOP with ERG rearrangement as well as a high correlation with $C H D 1$ deletion reinforces $S P O P$ as a distinct molecular subclass of prostate cancer.

895 MED15, Encoding a Subunit of the Mediator Complex, Is Overexpressed at High Frequency in Castration-Resistant Prostate Cancer $M$ Braun, Z Shaikhibrahim, R Menon, A Offermann, A Queisser, D Boehm, W Vogel, $K$ Ruenauver, C Ruiz, T Zellweger, M Svensson, O Andren, G Kristiansen, $N$ Wernert, L Bubendorf, S Perner. University Hospital Bonn, Bonn, Germany; University Bonn, Bonn, Germany; University Basel, Basel, Switzerland; St. Clara Hospital, Basel, Switzerland; University Orebro, Orebro, Sweden; CeGat, Tuebingen, Germany. Background: The Mediator, an evolutionarily conserved multi-subunit protein complex, is a key regulator of transcription of protein-coding genes, and an integrative hub for diverse signaling pathways. Here, we investigated whether Mediator, through subunits directly involved in signaling, is implicated in castration-resistant prostate cancer (CRPC) as a prototype of therapeutic-resistant diseases.

Design: Tissue microarrays (TMAs) were constructed from 651 prostate cancer (PCa) patients, comprising of 492 androgen-sensitive PCa, 89 distant metastatic CRPC (CRPC $\left.{ }^{\mathrm{MET}}\right)$ and 70 local recurrent $\left(\mathrm{CRPC}^{\mathrm{LOC}}\right)$. Mediator subunit 15 (MED15) was assessed for protein expression, amplification/rearrangement, and mutations by immunohistochemistry, fluorescence in-situ hybridization, and Sanger sequencing, respectively. TMAs were also assessed for TGFß-downstream target pSMAD3, and the proliferation markers Ki67 and PHH3. In PCa cell lines, MED15 was knocked down followed by proliferation assays with/without dihydrotestosterone (DHT), as well as treatment with recombinant TGF $\beta 3$.

Results: We show that MED15 is overexpressed in 76\% (53/70) of $\mathrm{CRPC}^{\mathrm{MET}}$ and $70 \%$ $(62 / 89)$ of CRPC ${ }^{\mathrm{LOC}}$, in contrast to $6 \%(29 / 492)$ in androgen-sensitive PCa. Further, we found $M E D 15$ to be amplified exclusively in $\mathrm{CRPC}^{\mathrm{MET}}$ and identified a recurrent somatic mutation in $\mathrm{CRPC}^{\mathrm{MET}}$. Moreover, $M E D 15$ overexpression correlated with worse clinical outcome. Further, high expression pSMAD3 correlated with MED15 overexpression in $\mathrm{PCa}$, and in-vitro activation of the TGF $\beta$ signaling lead to increased expression of MED15. Moreoever, knock-down of MED15 interfered with TGF $\beta$ signaling and decreased TGF $\beta$ signaling-driven proliferation. Finally, we found that MED15 overexpression is more common in $\mathrm{CRPC}^{\mathrm{MET}}$ than $A R$ overexpression, correlates with high proliferative activity, and knock-down of MED15 decreased androgen-dependent and -independent proliferation.

Conclusions: Our observations shed light into the potential role of MED15 in CRPC as a prototype of therapeutic-resistant diseases. We found MED15 overexpression to define a highly lethal phenotype and to interfere with TGF $\beta$ signaling. As MED15 is conserved, it is likely to emerge as a phenotype in other therapeutic-resistant diseases, and not restricted to our disease model.

896 Mixed Papillary Renal Cell Carcinoma (PRCC) Represents Similar Patterns of Chromosomal Abnormalities to Those of Pure Type 1 PRCC: A Study Including Nine Cases

F Brimo, RJM Eveleigh, $M$ Chevarie-Davis, $M$ Arsenault, $N$ Bertos, $S$ Tanguay, $M$ Latour, Y Riazalhosseini. McGill University Health Center, Montreal, QC, Canada; McGill University, Montreal, QC, Canada; Centre Hospitalier de l'Université de Montréal, Montreal, QC, Canada.

Background: PRCCs are classically divided into type 1 and type 2, However, many routine cases do not fulfill all the criteria for either type and display cytoplasmic or nuclear features of both types. In a previous work including 132 PRCCs, we have shown that the presence of high grade nuclear areas in tumors otherwise displaying features of type 1 PRCCs are of similar stage and immunoprofile as those with classic type 1 histology. We herein attempt to further confirm those findings at the molecular level using SNP array analysis using nine cases of PRCC.

Design: Included were paraffin-embedded tissue (FFPE) samples of three cases of pure type1, three of pure type 2, and three 'mixed' PRCCs showing type 1 morphology with variable high-grade areas (Fuhrman's grade 3 ). Macrodissection was performed from representative areas in each case and in the 'mixed'cases areas of different grades were analyzed separately. DNA was extracted from FFPE slices of each sample, and underwent restoration treatment prior to hybridization to SNP-arrays to interrogate approximately 750k SNPs in the human genome. Abnormal chromosomal patterns were detected using ASCAT (version 2.2) and GAP (Illumina September 2011 update) softwares.

Results: The whole-genome scan revealed recurrent gains at chromosomes 7 and 17 in all pure type 1 samples, whereas $2 / 3$ type 1 cases showed amplification of chromosome 16. Although two of the pure type 2 tumors were affected by focal amplifications on chromosome 17, this was not associated with entire chromosomal gains. In addition, type 2 tumors demonstrated focal deletion of chromosome $1 \mathrm{p}$ ( $3 / 3$ cases) as well as loss of chromosomes 14 and 15 (2/3 cases). Likewise, chromosome-level or focal amplifications of chromosome 8 was detected in pure type 2 samples, and not in pure type 1 specimens. Interestingly all specimens of mixed PRCCs (low- and high-grades) were affected by amplifications of chromosomes 7 and 17, and none exhibited chromosomal aberrations detected in pure type 2 tumors including loss of chromosomes 14,15 or gain of chromosome 8 .

Conclusions: This preliminary data confirm the fact that 'mixed' PRCC with focal high-grade areas have the same molecular signature as pure type 1 tumors suggesting that the spectrum of type 1 PRCCs is wider than originally described.

897 Pathological Predictors of Biochemical Recurrence in Patients with a Positive Surgical Margin at Radical Prostatectomy

L Bu, E Lucas, A Christie, D Khalil, Y Lotan, C Roehrborn, P Kapur. UT Southwestern Medical Center, Dallas, TX.

Background: To evaluate the relationship of pathologic tumor characteristics and biochemical recurrence after radical prostatectomy with a positive margin.

Design: We studied 104 radical prostatectomies done for pT2 or pT3a with positive surgical margins between 2006 and 2009. We assessed multiple pathological variables including gleason score (GS) of dominant nodule, stage, positive margin (PM) location, number of PM foci, linear extent of PM, highest Gleason grade at a PM and type of PM (extraprostatic extension (EPE) vs incisional). Univariate and multivariate analyses were performed using a Cox proportional hazards model to examine the relationships between these pathological variables and biochemical recurrence (BCR).

Results: Of the total 104 cases with PM (66 pT2+ cases and 38 pT3a cases), 30 patients had BCR over a follow-up period of XY months. BCR was significantly associated with higher stage, GS in dominant nodule, percentage of Gleason grade 4/5 in dominant nodule, larger tumor volume, presence of lymphovascular invasion, increasing number of EPE foci, linear length of EPE, more than 3 foci with PM, more than $3 \mathrm{~mm}$ linear length of PM, and presence of PM at EPE site (vs incisional PM). Multivariate analysis showed PM at EPE site and percentage of Gleason grade 4 and 5 tumor in dominant nodule as independent predictor for BCR. However, no BCR was observed in cases with PM of GS of 6. Additionally, tumor location, prostate volume, tertiary Gleason grade pattern, presence of benign prostatic glands at margin, or location of PM had no impact on $\mathrm{BCR}$.

Conclusions: EPE presence at PM and higher percentage of Gleason grade 4/5 in dominant nodule are independent pathological predictors for BCR after a RP with PM. These parameters should aid stratification of patients for adjuvant radiation therapy.

898 LIN28 Is More Sensitive Than SALL4 and OCT4 in Detecting Necrotic Embryonal Carcinoma and Seminoma

D Cao, X Rao, W Zhang, L Zhou, P Wang, L Jia. Peking University Cancer Hospital, Beijing, China; Peking University Shougang Hospital, Beijing, China; PLA 401 Hospital, Qingdao, China; Beijing Ditan Hospital, Beijing, China.

Background: Necrosis is relatively common in testicular embryonal carcinoma (EC) and less frequently in seminoma (SEM). Correct diagnosis and classification of germ cell tumors (GCTs) is critical for clinical management. In necrotic area, the cytologic features are obscured making it difficult to assign histological type just based on morphology, calling for immunohistochemical markers for this situation. Utility of immunohistochemical markers in classifying necrotic GCTs has been rarely studied. Here we investigated SALL4, OCT4 and LIN28 for their sensitivity in detecting necrotic EC and SEM.

Design: We only included testicular GCTS with both viable and necrotic areas next to each other to ensure that the histologic type in necrotic area can be assigned with certainty even without markers. Twenty-nine such cases (15 primary, 14 metastatic) were collected (21 ECs, 12 primary and 9 metastatic; 8 SEMs, 3 primary and 5 metastatic). One paraffin tumor block was used to generate 4 um unstained slides for immunohistochemical staining with SALL4, OCT4 and LIN28. In the necrotic area only distinct staining in a cellular distribution was considered positive. For SALL4 and OCT4 only nuclear staining was scored as positive and for LIN28 cytoplasmic staining was scored. The staining was scored semi-quantitatively as 0 (no tumor cells stained), $1+(1-30 \%), 2+(31-60 \%), 3+(60-90 \%)$ and $4+(>90 \%)$.

Results: The viable tumor cells in all 21 ECs and 8 SEMs showed $4+$ staining for SALL4, OCT4 and LIN28. The necrotic EC cells were positive for SALL4 in 19/21 $(90 \%, 1+$ in $13,2+$ in 6$)$, OCT 4 in $16 / 21(76 \%, 1+$ in $14,2+$ in 2$)$, and LIN 28 in $21 / 21$ $(100 \%, 2+$ in $5,3+$ in $5,4+$ in 11$)$ cases . The mean percentage of necrotic EC cells stained with LIN 28 , SALL4 and OCT4 was $79 \%, 16 \%$, and $14 \%$, respectively (LIN28 vs SALL4/OCT4, $\mathrm{p}<0.01$; SALL4 VS OCT4, $\mathrm{p}>0.05$ ). The necrotic SEM cells was positive for SALL4 in $6 / 8(75 \%, 1+$ in $5,2+$ in 1$)$, OCT4 in $6 / 8(75 \%, 1+$ in $5,2+$ in 1), and LIN28 in $7 / 8(88 \%, 3+$ in 3, 4+ in 4$)$ cases. Necrotic SEM cells were negative for all three markers in 1 case and were negative for both SALL4 and OCT4 in another. The mean percentage of necrotic SEM cells stained with LIN28, SALL4 and OCT4 was $90 \%, 15 \%$, and $12 \%$, respectively (LIN28 vs SALL4/OCT4, $p<0.01$; SALL4 VS OCT4, $\mathrm{p}>0.05$ ).

Conclusions: The necrotic EC and SEM cells showed much less staining for LIN28, SALL4 and OCT4 than viable cells. LIN28 is more sensitive than SALL4 and OCT4 in labeling necrotic EC and SEM.

899 Needle Biopsy in the Diagnosis of Renal Masses

$F$ Cate, A Coogan, P Clark, L Gellert, O Hameed, G Giannico. Vanderbilt Medical Center, Nashville, TN.

Background: Fine needle aspiration biopsy (FNA) with and without concurrent core needle biopsy $(\mathrm{CNB})$ offers a minimally invasive method to diagnose and assist in management of renal lesions. We aimed to determine the accuracy of FNA alone in the 
pathologic diagnosis of renal lesion compared to FNA and core needle biopsy (CNB). Design: Retrospective review of the cytopathology database for FNAs of renal lesions was performed from November, 2001 to April, 2013. The patient's medical records were reviewed to determine age, gender, size of nodule, number of needle passes, and adequacy assessment.

Results: 482 cases were identified including 79 FNAs, 58 FNAs with concurrent CNB, and 345 CNB. Only patients with FNA or FNA with CNB were analyzed. 48 patients were women (average age 63) and 90 were men (average age 65). The mean nodule size was $6.2 \mathrm{~cm}$. An average of 3 needle passes was performed. Of the $79 \mathrm{FNAs}$, 34 were deemed adequate on immediate assessment and 45 were inadequate. Final diagnoses were as follows: 17 non diagnostic, 3 angiomyolipoma, 2 cyst contents, 1 abscess, 11 oncocytic neoplasms, 40 renal cell carcinomas, and 5 metastatic malignancies. Of the 58 FNAs with concurrent CNB, 32 were deemed adequate on immediate assessment and 26 were inadequate. Final diagnoses were as follows: 9 non diagnostic, 1 primary neuroendocrine tumor of the kidney, 14 oncocytic neoplasms, 32 renal cell carcinoma and 1 metastatic malignancy. The non diagnostic rate, which included normal kidney, indeterminate or insufficient was $22 \%$ for FNAs vs. $15 \%$ for FNAs with concurrent CNB. 22 FNAs and 18 FNA with CNB had follow-up resection. Discordance between initial FNA assessment and final diagnosis was found in 2 cases of FNAs and 2 cases of FNA with CNB. In these cases, the specimen was inadequate on immediate assessment, showed normal kidney on $\mathrm{FNA} \pm \mathrm{CNB}$, and had a diagnosis of renal cell carcinoma on the final resection specimen. The accuracy of FNA alone to predict the final pathologic diagnosis was $100 \%$, while FNA with concurrent CNB predicted the final diagnosis with $100 \%$ accuracy. Further analysis of patients with CNB only is pending.

Conclusions: FNA with or without CNB is an appropriate diagnostic test for renal lesions. Although the accuracy rate of both is similar, FNA with concurrent CNB provides greater diagnostic material and a better chance for definitive diagnosis. Discordance between FNA or FNA with CNB diagnosis and resection diagnosis is commonly due to sampling error.

900 CDCA5 Overexpression as a Poor Prognostic Factor in Patients with Urothelial Carcinomas of Upper Urinary Tract and Urinary Bladder

I-W Chang, C-H Hung, C-F Li. Institute of Biotechnology and Chemical Engineering, I-Shou University, Kaohsiung, Taiwan; E-DA Hospital, Kaohsiung, Taiwan; Chi-Mei Medical Center, Tainan, Taiwan; NCRI, NHRI, Tainan, Taiwan.

Background: Despite advances in diagnostic imaging and treatment modalities, the risk stratification and final outcomes in patients with urothelial carcinomas (UC) of urinary bladder (UBUC) and upper urinary tract (UTUC) still remain suboptimal. Through data mining from a published transcriptomic database of UBUCs (GSE32894), cell division cycle associated 5 (CDCA5) was identified as the most significant gene showing stepwise upregulation during UBUC progression among those associated with G1-S transition of mitotic cell cycle (GO:0000082). CDCA5 gene encodes Sororin that regulates sister chromatid cohesion and is critical in mitosis. Given the roles of CDCA5 has not been investigated in UC, we therefore analyze its transcript and protein expressions and their associations with clinicopathological factors and survivals in our well-characterized cohort of UC

Design: Quantigene assay was used to detect $C D C A 5$ messenger RNA (mRNA) level in 40 UTUCs and 35 UBUCs, respectively. Immunohistochemistry evaluated by using $\mathrm{H}$-score was used to determine CDCA5 protein expression in $295 \mathrm{UBUCs}$ and 340 UTUCs, respectively. The mRNA and protein expression statuses were further correlated with clinicopathological features. The prognostic significance of CDCA5 protein expression was further evaluated for disease-specific survival (DSS) and metastasis-free survival (MeFS)

Results: Increment of $C D C A 5$ transcript level was associated with higher pT status and nodal metastasis in both UTUC and UBUC (all $\mathrm{p}<0.05$ ). CDCA5 protein overexpression was also significantly associated with advanced $\mathrm{pT}$ status (both $\mathrm{p}<0.001$ ), lymph node metastasis (UTUC, $\mathrm{p}<0.001$; UBUC, $\mathrm{p}=0.033$ ), high histological grade (both $\mathrm{p}<0.001$ ), vascular invasion (UTUC, $\mathrm{p}<0.001$; UBUC, $\mathrm{p}=0.045$ ), frequent mitoses (UTUC, $\mathrm{p}=0.002$; UBUC, $\mathrm{p}<0.001$ ) in both groups of UC. CDCA5 overexpression not only predicted worse DSS and MeFS at univariate analysis, but also implicated inferior DSS (both $\mathrm{p}=0.016$ ) and $\mathrm{MeFS}$ (UTUC, $\mathrm{p}=0.006$; UBUC, $\mathrm{p}<0.001$ ) in multivariate analysis. Conclusions: CDCA5 overexpression is associated with advanced tumor status and implicated adverse clinical outcome for both patients of UTUC and UBUC. Our study disclosed that CDCA5 plays an important role in tumor progression in UC and may serve as a potential prognostic biomarker and a novel therapeutic target of UC.

901 Molecular Pathways Altered in TMPRSS2/ERG Fusion Gene Expressing Prostate Cancers

D Chatterjee, G Ayala, M Ittmann. Baylor College of Medicine, Houston, TX; University of Texas Health Science Center Medical School Houston, Houston, TX.

Background: Prostate cancer remains the most common malignancy affecting men and the second leading cause of cancer-related death of men in the United States. It is a heterogeneous disease and the biology of various subtypes is still poorly understood. The TMPRSS2/ERG (T/E) fusion gene is present in more than $50 \%$ of PCas. Experiments in PCa cells containing the T/E fusion indicate that the TMPRSS2 promoter, which contains androgen receptor (AR)-responsive promoter elements, increase ERG expression in response to androgens. The ubiquitous activity of AR in PCa cells thus results in the constitutive expression of ERG fusion transcripts. Stable knockdown of the T/E fusion mRNA in PCa cells inhibits tumor growth in vivo. More recently we have shown that highly specific knockdown of the T/E fusion gene with siRNAS delivered via nanoliposomal vectors decreases growth of established tumors, confirming the importance of the $\mathrm{T} / \mathrm{E}$ fusion gene in PCa progression in vivo.

Design: The pathways and proteins implicated in the ability of the ERG oncoprotein to promote PCa progression has been a focus of active investigation.To better define the pathways associated with $\mathrm{T} / \mathrm{E}$ fusion gene expression we have analyzed correlations of T/E fusion gene expression with multiple key proteins associated with signal transduction and transcriptional control in PCa using a large tissue microarray with more than 500 prostate cancers. We analyzed expression of $\mathrm{T} / \mathrm{E}$ fusion protein using immunohistochemistry and then correlated expression with more than 200 markers that had previously been quantitatively analyzed in this same array.

Results: We found significant correlation with markers of activation of the PI3K pathway such as phospho-AKT and phospho-GSK and negative correlation with proteins that negatively regulate this pathway (PTEN and INPP4B) with T/E fusion gene expression. There was also a strong association with NFkB pathway activation. The MYC pathway was also upregulated in T/E fusion cancers. Increased expression of multiple transcription factors, including p53, serum response factor and SRC-1 coactivator were also significantly associated with fusion gene expression. Our results identify novel pathways not previously associated with T/E fusion gene expression that may play an important role in the biology of T/E fusion gene $\mathrm{PCa}$.

Conclusions: Expression of the T/E fusion gene is associated activation of multiple pathways contributing to $\mathrm{PCa}$ initiation and progression.

902 Minimal Prostate Cancer on TRUS Biopsy: Incidence and Follow-Up in a Cohort of 61 Patients

AR Chelliah, GD Stewart, MO'Donnell. Western General Hospital, Edinburgh, United Kingdom; Institute of Genetics \& Molecular Medicine, Edinburgh, United Kingdom. Background: Detection of early prostate cancer can be diagnostically and clinically challenging. Various terminology and criteria based on size and grade are used to report these small foci which may be missed or misdiagnosed as benign. Clinically, minimal cancer raises the question of whether to treat or opt for active surveillance making it important to identify clinicopathologic features that may distinguish indolent from more aggressive tumours. The aim of this study is to determine the incidence and outcome of minimal prostate cancer in our institute.

Design: A retrospective review was carried out of all primary prostatic adenocarcinomas diagnosed on 10 core TRUS biopsy between 2009-2011. Minimal cancer was defined as a small focus, $<2 \mathrm{~mm}$, present in 1 core without Gleason $4 / 5$ pattern. Patients already on active surveillance were excluded. Follow-up data, including serum PSA and repeat biopsy/prostatectomy findings, were obtained ranging from 20-44 months.

Results: 61 of 803 primary prostatic adenocarcinomas diagnosed in this period met our criteria for minimal cancer (7.6\%). 44 patients underwent active surveillance with PSA monitoring and/or annual repeat biopsy. 7 opted for radical prostatectomy after initial biopsy. 10 cases had no further record of biopsy/PSA monitoring. On repeat biopsy, 3 patients had upgrading of Gleason score/more extensive disease with rising PSA levels in 2 men. 11 men had minimal cancer and 22 men had no malignancy on repeat biopsies. This group had relatively stable PSA levels during active surveillance. 5 of the 7 resections had low-volume disease requiring deeper levels, immunostains and turning over of tissue megablocks to locate the tumour. The remaining 2 resections showed more extensive/higher-grade disease, with an anterior tumour in one which had not been sampled at biopsy. Significantly, this patient did not have a raised PSA at initial biopsy while 2 men with low-volume disease at surgery had raised PSA levels. Conclusions: The majority of patients with minimal cancer may be managed conservatively if PSA density remains low. Initially high or significantly rising PSA density during active surveillance should prompt further investigation. Importantly, initial biopsy findings/PSA levels did not correspond with resection findings in a small proportion of our patients emphasising the need for more sophisticated modalities to detect higher-risk disease, including pre-biopsy MRI to exclude anterior tumours, targeted biopsies and novel biomarkers.

903 Novel miRNA Expression Profiles and Potential Molecular Subtypes of Classical Seminoma Identified through Small RNA Sequencing

Y-T Chen, Z Chen, T Tuschl, N Renwick. Weill Cornell Medical College, New York, NY; Rockefeller University, New York, NY.

Background: Classical seminoma expresses a distinctive microRNA profile that reflects its derivation from pleuripotent stem cells, its germ cell differentiation, and evidence of malignant transformation. However, previous miRNA profiling studies on seminoma were based on micoroarray and/or PCR-based analyses and are limited by the pre-selected pool of miRNA species to be interrogated. In this study, we used small RNA sequencing to generate comprehensive miRNA expression profiles for seminoma, aiming at the identification of novel up-regulated miRNAs in this tumor.

Design: Total RNA was extracted from formalin-fixed paraffin-embedded tissue blocks of 18 classical seminomas and one spermatocytic seminoma. Small RNA sequencing was performed using an input of 100ng of total RNA, yielding an average of 3.4 millions miRNA sequence reads per sample. Unsupervised hierarchical clustering was performed using normalized miRNA counts, and lists of miRNA species that differentiated clusters were generated.

Results: Spermatocytic seminoma showed a miRNA profile distinctive from classical seminomas. All classical seminoma showed high level expression of miRNA-143, $-182,-92 \mathrm{a},-26 \mathrm{a},-29 \mathrm{a}, 148 \mathrm{a}$ and $-106 \mathrm{~b} / 25$ cluster etc., in addition to the previously known overexpression of miR-371-373 cluster and miR-21 etc.. Among these miRNAs previously unknown to be overexpressed in seminoma, miRNA-92a is part of the miR17-92a oncogenic cluster implicated in carcinogenesis of multiple human cancers, and miR-106b/25 cluster has also been shown to be up-regulated in cancer. Furthermore, unsupervised clustering separated the 18 classical seminomas into two molecular subgroups of 4 and 14 cases. Only 10 miRNAs showed statistically different expression among these two groups, the most prominent being 6 members of the miR-506-514 cluster on chromosome $\mathrm{X}$, showing up-regulation in the group of 4 cases ( $<<0.0001$ for all 6 members, with 13 to 20 fold differences). 
Conclusions: Direct miRNA sequencing allowed comprehensive unbiased miRNA profiling of classical seminoma and identified a panel of overexpressed miRNA species previously unidentified by microarray- and PCR-based methodologies. The newly identified up-regulated miRNAs included oncogenic miR-106b/25 cluster and miR92a. Moreover, our finding suggested two molecular subtypes of seminoma, with the main difference being the overexpression of miRs-506-514 cluster, a cluster of miRNA previously shown to be oncogenic in melanoma, in about $30 \%$ of cases.

\section{An Institutional Experience on the Management of Prostatic} Adenocarcinoma

$S$ Chen, J Fava, A Amin. Rhode Island Hospital, Providence, RI.

Background: Gleason score (GS) is an important factor in determining management and outcome of prostate adenocarcinoma. Despite introduction of the revised GS scheme by ISUP 2005 consensus conference, there is significant variation in scoring of prostate biopsy specimens among pathologists, especially between genitourinary (GU) and non-GU pathologists. In our practice, the biopsy material of all referal cases for definitive therapy are reviewed by a GU pathologist for confirmation of diagnosis and quality assurance.

Design: From 2011-2013, 117 consecutive prostate consults reviewed by one GU pathologist were collected including non-GU pathologist (initial) and GU pathologist consult (review) reports. Medical records were reviewed for follow up data and prostate specific antigen (PSA). The data was analyzed using Wilcoxon Signed Rank Sum Test. Results: Mean age of patients was 64 years (range: $46-89$ years). At arrival, $63 \%$ ( 74 cases) and $3 \%$ ( 3 cases) had one set (SB) and two sets (TB) of biopsies, respectively. Each case had from 6 to 16 cores $34 \%$ (40 cases) had a single biopsy prior to radical prostatectomy (RP). In SB cases, $56.7 \%$ (42) had followup data for treatment. Of these, $78.5 \%$ (33) received radiation and/or androgen therapy and $19 \%(8)$ received active surveillance. The initial GS was higher for all specimens $(\mathrm{p}=0.007)$ especially for the $R P$ cases $(p=0.002)$. Overall, the modal GS on initial diagnosis was $4+3=7$ that was downgraded to the modal GS of $3+3=6$ upon review. Despite an overall substantial agreement $[\mathrm{ICC}=0.62]$ between the non-GU and $\mathrm{GU}$ pathologists, $\mathrm{GS}$ by $\mathrm{GU}$ pathologist tended to have a better correlation [ICC: 0.62 ] than non-GU pathologist [ICC: 0.48 ] with the final GS in RP specimens. GS showed significant correlation with pre-operative PSA only in GU pathologist review ( $\mathrm{p}=0.002)$. Post treatment PSA data was retrieved in 19 RP specimens with $95 \%$ (18) maintaining a PSA $<1.0 \mathrm{ng} / \mathrm{ml}$ (range: $0.03-0.1 \mathrm{ng}$ / $\mathrm{ml}$ ). Post treatment PSA data was retrieved in $45 \%$ (33) SB specimens with $36 \%$ (12) having post treatment values of $>1.0 \mathrm{ng} / \mathrm{ml}$ (range: $1.2-35.0 \mathrm{ng} / \mathrm{ml}$ ).

Conclusions: This study demonstrates that the non-GU pathologist is likely to assign a higher GS than the GU pathologist, which may have therapeutic implications. Also scoring by the GU pathologist is more likely to correlate with the GS at prostatectomy and pre-operative PSA. Our findings are also in keeping with the current literature on the usefulness of PSA for monitoring post treatment patients with prostatectomy and radiation and/or androgen deprivation.

905 Hereditary Leiomyomatosis and Renal Cell Carcinoma (HLRCC)Associated Renal Cancer: The Utility of Detecting Aberrant Succination by Immunohistochemistry

Y-B Chen, AR Brannon, ME Dudas, $H$ Won, HA Al-Ahmadie, SW Fine, A Gopalan, $N$ Frizzell, P Russo, MF Berger, SK Tickoo, VE Reuter. Memorial Sloan-Kettering Cancer Center, New York, NY; University of South Carolina, Columbia, SC.

Background: HLRCC is an autosomal dominant disorder in which germline mutations of fumarate hydratase $(\mathrm{FH})$ gene confer an increase risk of cutaneous and uterine leiomyomas as well as renal cancer. HLRCC-associated RCCs are highly aggressive, frequently presenting as a solitary mass, and histologically showing a spectrum of architectural patterns. While HLRCC tumor exhibits distinctive viral inclusion-like prominent nucleoli and peri-nucleolar halo, distinguishing it from various types of highgrade sporadic RCCs with prominent nucleoli is challenging. High level of fumarate accumulated in HLRCC tumor cells has been found to cause aberrant succination of cellular proteins. This modification, S-(2-succino)-cysteine (2SC), has been reported to be detectable by immunohistochemistry (IHC).

Design: IHC for 2SC was performed in 10 genetically confirmed HLRCC-associated RCCs, multiple tissue microarrays and whole tissue sections from 184 clear cell, 45 type 2 papillary, 97 high-grade unclassified, 2 TFE3 translocation-associated RCCs and 2 renal medullary carcinomas. To examine the correlation between $2 \mathrm{SC}$ IHC and $\mathrm{FH}$ mutations, we analyzed the $\mathrm{FH}$ gene status in 16 of these tumors using a targeted next-generation exome sequencing platform that evaluates mutations and copy number alterations.

Results: All confirmed HLRCC tumors (10/10) demonstrated diffuse and strong nuclear and cytoplasmic staining for 2SC, while the adjacent renal parenchyma was negative. In contrast, all clear cell (184/184), 96\% (93/97) of high-grade unclassified RCC, and the large majority of type 2 papillary (35/45, 78\%) cases showed no $2 \mathrm{SC}$ immunoreactivity. The TFE3-translocation and renal medullary carcinomas were also negative. A subset of papillary $(10 / 45,22 \%)$ and unclassified $(4 / 97,4 \%)$ tumors showed patchy or diffuse cytoplasmic staining, which was distinct from the nuclear and cytoplasmic pattern seen with confirmed HLRCC tumors. Sequencing revealed no germline or somatic $\mathrm{FH}$ alterations in 3 unclassified and 2 papillary RCCs that exhibited diffuse or patchy $2 \mathrm{SC}$ cytoplasmic staining. Eight cases negative for $2 \mathrm{SC}$ but showing HLRCC-like nuclear features also lacked $F H$ alterations. In comparison, the 3 HLRCC tumors with $2 \mathrm{SC}$ nuclear and cytoplasmic staining harbored somatic $\mathrm{FH}$ mutations or loss of heterozygosity in addition to germline $\mathrm{FH}$ mutations.

Conclusions: Our results indicate that detection of $2 \mathrm{SC}$ by IHC is a useful ancillary tool in the differentiation of HLRCC renal tumors from other high-grade RCCs.
906 Expression of de Novo Lipogenic Enzymes and Prostate Cancer Progression after Radical Prostatectomy

$J$ Cheng, B Xu, H Huang, C Gao, $W$ Chen, JL Mohler, JR Marshall. Roswell Park Cancer Institute, Buffalo, NY; University at Buffalo, Buffalo, NY.

Background: A body of evidence indicates that aberrant lipid metabolism is involved in the pathogenesis of prostate cancer (PCa). However, the degree to which deregulated lipid metabolism is associated with prostate cancer progression is still elusive. This study analyzes the expression pattern of several key enzymes in de novo lipogenesis and evaluates the predictive role of lipogenic gene expression signatures in postprostatectomy outcomes.

Design: Paraffin-embedded tissues from 707 patients who had radical prostatectomy (RP) at our institute, between 1993 and 2005, were used for TMA construction. The protein expression levels of fatty acid synthase (FASN), Acetyl-CoA carboxylase (ACC), Stearoyl-CoA desaturase-1 (SCD1) and Ki-67 were assessed by IHC staining. The patients were followed for a median of 9.4 years. Treatment endpoints included biochemical recurrence, treatment failure and metastatic $\mathrm{PCa}$. Cox proportional hazards analyses were used to evaluate the associations of lipogenic enzyme expression with each endpoint. Bivariate associations with outcomes were considered; the associations were also controlled for established outcome predictors, including age at surgery, BMI, PSA, pathologic stage and grade and margin status.

Results: While PCa tissue expressed higher levels of FASN and ACC than benign prostate epithelium, the expression of SCD1 in neoplastic tissue was lower than that in benign tissue. About $16 \%$ of patients in the cohort had neo-adjuvant androgen deprivation therapy (ADT); FASN stain in patients with ADT was slightly decreased in PCa tissue, compared to that in non-ADT patients (2.22 vs. $2.38, \mathrm{p}=.027)$; ACC stain in PCa tissue was higher in patients with ADT (1.31 vs. 1.02, $\mathrm{p}<.001)$ and SCD1 stain in PCa tissue was comparable for ADT-patients and non-ADT patients ( $1.10 \mathrm{vs.}$ $0.99, \mathrm{p}>.05$ ). In patients who did not receive ADT, the staining intensities of FASN, $\mathrm{ACC}$ and SCD1 were positively associated with $\mathrm{Ki}-67$ proliferation index in the $\mathrm{PCa}$ tissue, but no significant association between the expression of each lipogenic enzyme and the outcomes after RP was found. However, the expression of FASN (+) ACC (+) and SCD1 (-), representing $11 \%$ of non-ADT patients, was associated with increased risk of treatment failure (adjusted $\mathrm{HR}=2.03, \mathrm{p}=.037$ ).

Conclusions: De novo lipogenic gene expression signature may be a predictor of post RP outcome.

907 Inflammatory Myofibroblastic Tumor of the Urinary Bladder: The Role of IgG4 and the Comparison of Two Immunohistochemical Antibodies and Fluorescence In-Situ Hybridization in the Detection of ALK Alterations E Choi, SR Williamson, $M$ Wang, JN Eble, DJ Grignon, R Montironi, MT Idrees, $S$ Zhang, LA Baldridge, M Scarpelli, GT MacLennan, A Lopez-Beltran, AO Osunkoya, $L$ Cheng. Indiana University School of Medicine, Indianapolis, IN; Indiana University Health, Indianapolis, IN; Polytechnic University of the Marche Region and United Hospitals, Ancona, Italy; Case Western Reserve University, Cleveland, OH; Henry Ford Health System, Detroit, MI; Cordoba University, Cordoba, Spain; Emory University, Atlanta, GA

Background: We studied $A L K$ gene rearrangement and the expression of ALK protein in urinary bladder inflammatory myofibroblastic tumor (IMT) using fluorescence in-situ hybridization (FISH) and two immunohistochemical (IHC) antibodies to ALK. We also investigated whether IMT represents an IgG4-related systemic disease (IgG4-RSD). Design: We examined ALK alterations by IHC and FISH in 15 patients with IMT of the urinary bladder. IHC was also utilized to quantitate intratumoral IgG4-positive plasma cells. The performance characteristics of the Dako FLEX monoclonal antibody (CD246) and the Cell Signaling Technology (D5F3) XP monoclonal antibody to ALK were compared.

Results: Of 15 tumors, nine showed ALK expression by IHC (60\%). Similar positive reactivity was observed with both antibodies, although the Cell Signaling Technology D5F3 antibody yielded a stronger intensity of staining in 4 of the 9 positive cases. By FISH, 7 of 15 tumors demonstrated $A L K$ rearrangements (47\%). Two tumors were ALK-positive by IHC but negative for rearrangement by FISH, whereas one showed $A L K$ rearrangement by FISH but was negative by IHC. Using the most current criteria, no cases met the specifications for classification as an IgG4-RSD.

Conclusions: The Cell Signaling Technology ALK (D5F3) IHC antibody showed good correlation with the Dako FLEX monoclonal antibody (CD246) and showed a stronger intensity of staining in a subgroup of positive cases. Discordant results between ALK IHC and $A L K$ FISH suggest a role for post-transcriptional modification and other levels of regulation in gene expression. Therefore, for diagnostically challenging cases with a negative result by IHC or FISH, supplementation with the other technique may be helpful in supporting the diagnosis. Finally, by current criteria, IMT does not represent an IgG4-RSD.

908 Nerve, Vessel, and Soft Tissue Damage Following Serial Prostate Biopsies: A Retrospective Analysis

KM Clark Schneider, TD Trainer. Fletcher Allen Health Care, Burlington, VT

Background: On review of total prostatectomy specimens from men who have undergone serial prostate biopsies, pathologists at our institution have noted injury of peri-prostatic nerves and blood vessels, as well as inflammation and fibrosis of the peri-prostatic soft tissue. As active surveillance becomes more routine in men with low-volume, low-grade prostate cancer, it is necessary to investigate any potential side effects of serial prostate biopsies.

Design: We retrospectively reviewed H\&E-stained sections of peri-prostatic soft tissue from all total prostatectomy specimens submitted at our institution from 2009 to 2011 (74 cases). The assessed parameters included peri-prostatic stromal inflammation, 
fibrosis, and hemosiderin deposition; arteritis; phlebitis; nerve inflammation; and ganglion inflammation and degeneration. The severity of these findings was recorded on a scale of 0 (absent) to $3+$ (marked).

Results: Ganglion degeneration was the most common abnormality, ranging from subtle changes such as hyperchromasia and cytoplasmic granularity $(1+)$ to marked damage including nuclear pyknosis and cytoplasmic vacuolation (3+). Stromal hemosiderin deposition was also common. In some cases, the hemosiderin followed an obvious needle track, favoring its relationship to a prior biopsy. Inflammation of both nerves and stroma was identified in over half of the specimens.

\begin{tabular}{|c|c|c|c|c|c|}
\hline 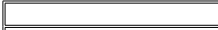 & $1+$ & $2+$ & $3+$ & Total \# of Specimens & Percentage $(n=74)$ \\
\hline Ganglion Degeneration & 7 & 55 & 111 & 73 & $99 \%$ \\
\hline Ganglion Inflammation & 8 & 4 & 0 & 12 & $16 \%$ \\
\hline Stromal Hemosiderin & 36 & 29 & 1 & 66 & $89 \%$ \\
\hline Stromal Inflammation & 27 & 14 & 4 & 45 & $61 \%$ \\
\hline Stromal Fibrosis & 15 & 8 & 0 & 23 & $31 \%$ \\
\hline Nerve Inflammation & 28 & 17 & 1 & 46 & $62 \%$ \\
\hline Arteritis & 16 & 3 & 1 & 20 & $27 \%$ \\
\hline Phlebitis & 15 & 6 & 0 & 21 & $28 \%$ \\
\hline
\end{tabular}

Conclusions: The common presence of peri-prostatic tissue injury in prostatectomy specimens suggests that prostate needle biopsies are not altogether benign procedures. The clinical significance of these findings is uncertain; however, the nearly universal finding of ganglion degeneration is noteworthy, given the location of nerves associated with penile erectile function in the peri-prostatic soft tissue. Of the 74 cases, only 9 men had undergone more than one prior biopsy; therefore, it is unclear whether these injuries would increase in amount and/or severity with repeat biopsies. As active surveillance of low-grade, low-volume prostate cancer becomes increasingly routine, continued analysis of prostatectomy specimens will aid in determining whether repeat biopsies are associated with worsening peri-prostatic tissue injury, and may identify any association of these findings with clinical symptoms.

909 Staging of Bladder Cancer (PTA VS. PT1) among 238 European Pathologists Reporting pTa Versus pT1 on Transuretheral Resection Specimen of the Bladder (TURB). A Survey of 238 European Pathologists E Comperat, L Egevad, A Lopez-Beltran, P Camparo, F Algabe, M Amin, J Epstein, $H$ Hamberg, C Hulsbergen-Van de Kaa, G Christiansen, R Montironi, C-C Pan, Tvan der Kwaast. Hopital La Pitie Salpetriere, Paris, France.

Background: The assessment of pTa versus pT1 in bladder cancer based on transuretheral resection specimens of the bladder (TURB) is challenging both for expert uropathologists (EUP) and general pathologists (GP). There is still a lack of consensus in this clinically important decision.

Design: A web-based survey was distributed among 661 members of the European Network of Uropathology (ENUP). Twelve cases that were previously circulated amongst 8 international experts were presented by 4 microphotographs for assessment of stage. Consensus was defined as $2 / 3$ agreement.

Results: 238 pathologists from 20 European countries replied. Consensus was reached amongst both EUP and GP in 5 cases (1 pTa and 4 pT1) and amongst EUP only in 2 cases (1 pTa, $1 \mathrm{pT} 1)$. The most common findings amongst GP in favor of pT1 were small nests of tumor cells in close association with suburothelial capillary network (44\%), on the contrary to EUP, followed by rounded and well demarcated nests of tumor cells in the lamina propria (29\%) and single nests in the lamina propria close to the overlying urothelium $(24 \%)$. The most common findings in favor of pTa were features of tangential cutting of nests $(73 \%)$ and individual rounded and well-demarcated nests of tumor cells in the lamina propria and confluent nests (26\%). The most common reasons for uncertainty were lack of spatial orientation and artifacts. One case achieved consensus among GP (1 pT1), but not among EUP because of small nests $(59,4 \%)$ single attached cells dissociating from urothelial nests $(45.1 \%)$ and single detached cells in the lamina propria (39.4\%). In 4 of 5 cases for which EUP could not reach consensus GP were also unable to agree.

Conclusions: The diagnosis of $\mathrm{pTa} / \mathrm{T} 1$ is still challenging for pathologists. It is easier to reach consensus on stage in $\mathrm{pT} 1$ bladder cancer than in $\mathrm{pTa}$. A teaching set of images for standardization of histological criteria for invasiveness of bladder cancer might be helpful to improve interobserver reproducibility.

910 GATA3 Is a Sensitive and Relatively Specific Biomarker for Testicular Choriocarcinoma

LA Compton, M Buchanan, MS Hirsch. Brigham and Women's Hospital, Boston, MA. Background: The majority of testicular germ cell tumors (GCTs) can be diagnosed purely by histologic examination. However, in a subset of cases the distinction of malignant germ cell components can be difficult, requiring the use of immunohistochemical (IHC) stains. Although reliable markers are available for embryonal carcinoma (EC) and seminoma, similar useful biomarkers with high sensitivity and specificity for yolk sac tumor (YST) and choriocarcinoma have not been identified. GATA3 is a zinc finger transcription factor which plays an important role in testicular gonadogenesis and trophoblastic differentiation. Studies have shown that GATA3 is expressed in breast and urothelial carcinomas, and is absent in EC, seminoma and YST, among other carcinomas; however, choriocarcinoma was not included in this series. The goal of this study was to evaluate the utility of GATA3 as a biomarker for choriocarcinoma. Design: 40 cases were stained with GATA3, including 34 primary GCTs, 4 metastatic testicular GCTs, 1 primary mediastinal GCT and 1 testicular B-cell lymphoma. Primary GCTs included 10 pure seminomas, 3 pure choriocarcinomas, 1 pure EC, 1 pure YST, 1 pure teratoma, 21 mixed GCTs, and 2 spermatocytic seminomas. Syncytiotrophoblasts in the absence of choriocarcinoma were noted by H\&E. In a subset of mixed GCTs OCT3/4, AFP and glypican were utilized to confirm areas of EC and YST. Only nuclear staining was considered a positive result.
Results: All tumor associated syncytiotrophoblasts and choriocarcinomas (primary and metastatic) were strongly and diffusely positive for GATA3. In contrast, pure and mixed areas of EC $(n=15)$ and seminoma $(n=14)$ were always GATA3 negative. GATA3 was focally positive in a subset of YSTs $(6 / 11,55 \%)$ with weak to moderate staining. Half $(5 / 10)$ of the teratomatous components, including both mature and immature elements, displayed focal weak to moderate GATA3. Both spermatocytic seminomas and the B-cell lymphoma were GATA3 negative.

Conclusions: GATA3 is strongly and diffusely positive in cytotrophoblasts and syncytiotrophoblasts in the testis, and can reliably be used to distinguish choriocarcinoma from other malignant germ cell elements, especially EC and seminoma. GATA3 can also be used to identify isolated syncytiotrophoblasts in GCTs in the absence of choriocarcinoma, and to confirm metastatic choriocarcinoma in distant sites. Overall, the additional use of GATA3 in combination with other reliable GCT markers such as Oct3/4 and SALL4 will allow for more accurate subclassification of GCTs, which ultimately can have an effect on patient management.

911 Papillary Renal Cell Carcinoma: Correlation of Tumor Grade and Histologic Characteristics with Clinical Outcome

KM Cornejo, F Dong, C-L Wu, RH Young, K Braaten, PM Sadow, GP Nielsen, E Oliva. Massachusetts General Hospital, Boston, MA

Background: Histologic prognostic parameters in papillary renal cell carcinoma (PRCC) are unclear, and few consistently show a significant correlation with survival. Recently, the validity of Fuhrman and nucleolar grading in PRCC as prognostic indicators has been questioned. The aim of our study is to review the clinicopathologic features of PRCC, including Fuhrman and nucleolar grade, to determine which parameters are independent prognostic indicators.

Design: Cases of PRCC treated by radical or partial nephrectomy were retrieved from the surgical pathology files from 1984-2010. Parameters studied included tumor multifocality, size, PRCC type (1 or 2), cytologic features including Fuhrman and nucleolar grade, necrosis, foamy macrophages, lymphovascular invasion (LVI) and pTNM stage at presentation to cancer-specific survival (CSS). The nucleolar grade was determined by nucleolar prominence in a high-power field (400X) containing the greatest degree of nuclear pleomorphism in which absent or inconspicuous nucleoli is grade 1 , nucleoli visible at $400 \mathrm{X}(10 \mu \mathrm{m})$ is grade 2 , and nucleoli easily visible at $100 \mathrm{X}(15 \mu \mathrm{m})$ is grade 3 .

Results: Of 154 PRCC, 112 (73\%) were type 1 and 42 (27\%) type 2 . One hundred twenty-five patients were males and 29 were females with age at presentation varying from 26-86 (mean 62.7) years. Tumors measured $0.4-17.0 \mathrm{~cm}($ mean $5.1 \mathrm{~cm})$ in size and the mean follow-up interval was $73.9(0.13-222)$ months. Univariate analysis identified nucleolar grade $(\mathrm{P}=0.0001)$, Fuhrman grade $(\mathrm{P}<0.0001)$, size $(\mathrm{P}<0.0001)$, $\mathrm{PRCC}$ type $(\mathrm{P}=0.03)$, tumor stage $(\mathrm{P}<0.0001)$, nodal stage $(\mathrm{P}<0.0001)$, necrosis $(\mathrm{P}=0.03)$ and LVI $(\mathrm{P}<0.0001)$ as prognostic factors of CSS. On multivariate analyses, only tumor size (hazard ratio, HR 3.73, $\mathrm{P}=0.0003$ ), LVI (HR 32.6, $\mathrm{P}<0.0001$ ), and Fuhrman (HR 9.07, $\mathrm{P}=0.005$ ) or nucleolar grade (HR 7.9, $\mathrm{P}=0.005$ ) were predictors of survival.

Conclusions: In this study, both Fuhrman and nucleolar grading systems are highly predictive of prognosis in patients with PRCC. Therefore, either grading system may be used on patients with PRCC. Other prognostic factors include tumor size, LVI and PRCC type, the latter only by univariate analysis.

912 Adult Granulosa Cell Tumors of the Testis: A Case Series of 31 Patients

KM Cornejo, RH Young. Massachusetts General Hospital, Boston, MA.

Background: Little is known about adult granulosa cell tumors (AGCT) of the testis compared to the ovarian counterpart and the more common sertoli cell tumor of the testis. Their morphologic spectrum and behavior are unclear; the largest series was comprised of 7 cases with the remaining being mainly case reports. The aim of our study is to review the clinicopathologic features of AGCT of the testis, to better understand their pathology and malignant potential.

Design: A total of 31 cases of AGCT were seen in consultation at our institution between 1994-2012. Histologic features such as tumor patterns, cytologic features including mitotic count, and the presence of lymphovascular invasion (LVI) or necrosis were analyzed, in addition to the clinical history and gross description when available. Results: The patients were from 14 and 87 years of age (mean 39.6 years) and all underwent orchiectomy or wedge excision. None of the patients had endocrinerelated symptoms or elevated serum markers. Follow-up was available for 16 of the 31 cases. The mean follow-up interval was 48.2 months (1-169 months). All patients were without evidence of disease except one who had lung metastases at 24 months. The tumors measured 0.5 to $6.0 \mathrm{~cm}$ (mean $2.8 \mathrm{~cm}$ ) in size and were predominantly well-circumscribed, and yellow-tan, except for one case which had infiltrative borders. Histologically, they showed the various typical patterns of AGCT of the ovary. The predominant morphology was diffuse, but spindled, insular, trabecular, microfollicular, watered-silk and cystic patterns were also noted. Call-Exner bodies and luteinization were present in 5 and 3 cases, respectively. Six cases with a spindled pattern had prominent fibromatous stroma giving a fibrosarcoma-like morphology. The mitotic count ranged from 0-18/10 high-power fields (HPF) (mean 4.8/10 HPF, 400X). Two cases had prominent necrosis and LVI was noted only in the case with metastases.

Conclusions: Our findings indicate that the morphologic spectrum of testicular AGCT is similar to that of ovarian AGCT. The majority of AGCTs of the testis have a good prognosis (compared with 2 of 7 cases with malignant behavior, previously reported in one series). Conventional features such as LVI and infiltrative borders may help in identifying cases with more aggressive behavior, as both features were present in the one case with metastases in our series. Mitotic counts vary and do not appear to be prognostic, although data is still limited. 
913 Hilar Soft Tissue Involvement by Testicular Germ Cell Tumors Is Associated with Advanced Stage Indicators

JM Cortazar, BE Howitt, E O'Donnell, C Sweeney, T Ulbright, MS Hirsch. Brigham and Women's Hospital, Boston, MA; Dana-Farber Cancer Institute, Brookline, MA; Indiana University, Bloomington, IN

Background: Testicular germ cell tumors (GCT) are divided into 2 major categories: seminomatous and nonseminomatous (NSGCTs). Current pathologic staging does not include invasion into testicular hilar soft tissue. Recently a series of NSGCTs demonstrated that hilar fat involvement is more frequently associated with metastases. The goals of this study were to validate the significance of hilar soft tissue invasion by NSGCTs and to include an evaluation of pure seminomatous GCTs.

Design: Radial orchiectomy cases with (experimental cohort, $n=106$ ) and without (control cohort, $\mathrm{n}=106$ ) hilar soft tissue involvement were retrospectively evaluated for: age at diagnosis, tumor subtype, invasion into hilar soft tissue, size, focality, lymphovascular invasion (LVI), rete testis (RT) invasion, epididymis invasion, and clinical follow up. In each cohort the cases included 47 pure seminomas, 9 pure embryonal carcinomas, and 50 mixed germ cell tumors. Clinical parameters between cohorts were compared using Fisher exact test.

Results: There was no significant difference in patient age or mean tumor size between experimental and control cohorts (33 vs. 36 and 4.1 vs. $3.7 \mathrm{~cm}$ ). Hilar soft tissue invasion in GCTs of all types (mixed and pure) demonstrated a significant association with LVI $(p<0.0001)$, RT invasion $(p<0.0001)$, epididymis invasion $(p<0.0001)$, and future metastatic disease $(\mathrm{p}=0.013)$. A trend towards involvement of retroperitoneal lymph nodes (RPLND) by tumor was observed, but this was not statistically significant $(\mathrm{p}=0.07)$. Among pure seminomas, those with hilar fat invasion demonstrated a significant association with LVI $(\mathrm{p}=0.025)$, RT invasion $(\mathrm{p}<0.0001)$, and epididymis invasion $(\mathrm{p}<0.0001)$. Mixed GCTs were significantly associated with LVI $(\mathrm{p}<0.0001)$ and RT invasion $(\mathrm{p}=0.0006)$, with a trend towards an increased likelihood of a node positive RPLND ( $p=0.07$ ). There were too few pure embryonal carcinomas to compare, and there were no reported deaths from disease.

Conclusions: Hilar soft tissue invasion in radial orchiectomy specimens for GCTs of all types (pure and mixed) is associated with increased incidence of RT invasion, epididymis invasion, LVI, and metastatic disease. Pure seminomas and mixed GCTS maintain an association with LVI and RT involvement; however a significant association with metastatic disease is less certain. Nevertheless, this study confirms that hilar fat invasion in radial orchiectomy specimens may predict advanced stage.

914 Does the Mitotic Rate in Non-Invasive Low-Grade Papillary Urothelial Carcinoma (LG) Carry a Prognostic Significance?

$S$ Dauphin-Pierre, W Kassouf, F Brimo. McGill University Health Center, Montreal, QC, Canada

Background: Although the classic description of LG includes the presence of scattered rare mitoses, what constitutes 'rare mitoses' is not defined in the literature. As some typical LGs in terms of cytologic atypia show abundant mitotic activity, we have sought to investigate the prognostic significance of the number of mitoses in a cohort of 91 LG with long term follow-up.

Design: 91 LGs with no previous history of higher grade urothelial lesions were included. The highest number of mitoses per HPF was recorded along with other pathological parameters such as tumor size, number of tumor blocks, presence/absence of necrosis, and correlated with tumor recurrence and progression. Brisk mitotic activity was defined as $>5$ mitoses / 1HPF. Cases with brisk mitoses in more than one HPF were labelled as having 'multifocal brisk mitoses'

Results: 13 cases were upgraded and two had recurrence less than three months from the diagnosis and were excluded. Of the included 76 patients, $82.9 \%$ were male and the mean age of 73 years. Mean follow-up time was 32 months. Six LGs $(8 \%)$ showed brisk mitotic activity and six ( $8 \%$ ) showed necrosis. Overall, progression occurred in $15.8 \%$ of cases $(10.5 \%$ grade progression and $5.3 \%$ stage progression) at a mean time of 22 months. Presence of necrosis in an otherwise typical LG showed comparable rates of progression to the entire cohort (17\%). In comparison, cases with brisk mitoses had significantly higher progression rates than those with rare mitoses (33\% versus $14.3 \%$, respectively). Furthermore, two out of the three cases with multifocal brisk mitoses progressed. Univariate analysis showed strong correlation between progression and the number of mitosis/HPF ( $p=0.05)$, multifocal brisk mitoses $(p=0.01)$, and the number of tumor blocks $(\mathrm{p}=0.03)$ and no correlation with gross tumor size $(\mathrm{p}=0.18)$, necrosis $(\mathrm{p}=0.74)$ or the presence of a previous history of $\mathrm{LG}(\mathrm{p}=0.17)$.

Conclusions: A tendency to underdiagnose high-grade papillary urothelial carcinoma persists. A minority of LGs show brisk mitotic activity defined as the presence of $\geq 5$ mitoses / 1HPF. Those lesions seem to be associated with higher progression rates than typical LGs, and the outcome is worse for cases in which this finding is multifocal within the tumor. If confirmed in larger series, those preliminary findings indicate that the presence of brisk mitotic activity in an otherwise typical LG should probably be included in the final pathology report and its association with a potential worse outcome should be noted.

$915 \quad$ Highest Percentage of Core Involvement by Gleason Score 7 (\%GS7) Is an Independent Predictor for pT3 Tumor on Radical Prostatectomy (RP) in Patients Diagnosed with Gleason Score 7 (GS7) Prostatic Carcinoma (PCa) on Biopsy

$B$ Dave, L Quintana, A Ward, B Xu, F Brimo, $H$ Ye. Beth Israel Deaconess Medical Center, Boston, MA; McGill University, Montreal, Canada.

Background: According to the D'Amico criteria, GS7 PCa diagnosed on core biopsy is considered high risk if the highest $\%$ of core involvement by any Gleason score ( $\% \mathrm{Ca}$ ) is $\geq 50 \%$ even if the PSA is $<20 \mathrm{ng} / \mathrm{ml}$. However, those "high risk" cases are often found to be $\mathrm{pT} 2$ on RP. This study aimed to evaluate additional features in GS7 biopsies that can be useful in predicting RP outcome.

Design: 186 consecutive RP cases following GS7 diagnosed on core biopsy were collected from 2007 to 2012. Biopsy and RP slides were reviewed by two GU pathologists using the modified Gleason grading system. The number and highest $\%$ of positive cores involved by a) $\mathrm{PCa}$ of any grade, and b) GS7 PCa, as well as morphological subtypes of Gleason pattern 4, were evaluated and correlated with findings at RP.

Results: The rates of advanced stage ( $\geq \mathrm{pT} 3)$, positive margin, and nodal involvement were $38.7 \%, 36.6 \%$, and $1.7 \%$, respectively. PSA and $\%$ GS7 were found to be significantly associated with advanced stage on RP in both univariate $(\mathrm{p}=0.0000$ and 0.0001 , respectively) (Table1) and multivariate analyses ( $\mathrm{p}=0.000$ and 0.041 , respectively) (Table2). In comparison, the ratio of GS7 cores / total number of cores (\#GS7/\#total) was significant on univariate but not multivariate analysis (Table 1\&2). Of patients with PSA $<20 \mathrm{ng} / \mathrm{ml}$ and $\% \mathrm{Ca} \geq 50 \%$ on biopsy, $43.6 \%$ (51 of 117 ) had advanced stage on RP. This "high risk" group could be further divided into two risk groups based on $\% \mathrm{GS} 7$ on biopsy: while pT3 rate was $21.9 \%(7 / 32)$ if $\% \mathrm{GS} 7 \leq 50,51.8 \%$ $(44 / 85)$ of patients had advanced stage when $\% \mathrm{GS} 7>50(\mathrm{P}=0.004$, Fisher's exact test $)$. Table 1. Univariate analysis of pre-RP characteristics predicting $\mathrm{pT} 3$

\begin{tabular}{|c|c|c|c|}
\hline Variables & Odds ratio & 95\% Confidence interval & P-value \\
\hline PSA & 1.25 & $1.13-1.39$ & 0.0000 \\
\hline \%GS7 & 1.02 & $1.01-1.03$ & 0.0001 \\
\hline \#GS7/\#Total & 12.16 & $1.97-75.11$ & 0.0058 \\
\hline
\end{tabular}

Table 2. Multivariate analysis of pre-RP characteristics predicting $\mathrm{pT} 3$

\begin{tabular}{|l||l|l|l||}
\hline Variables & Odds ratio & 95\% Confidence interval & P-value \\
\hline PSA & 1.23 & $1.11-1.37$ \\
\hline \%GS7 & 1.01 & $1.00-1.03$ & 0.000 \\
\hline \#GS7/\#Total & 1.57 & $0.17-14.54$ & 0.041 \\
\hline
\end{tabular}

Conclusions: If confirmed in a larger cohort, the findings from the current study indicate that $\% \mathrm{GS} 7$ could be a better risk predictor for RP outcomes than $\% \mathrm{Ca}$ in patients with GS7 PCa detected on biopsy. We are in the process of evaluating whether different Gleason patterns 4 on biopsy carry different prognostic values.

916 Length and Extracapsular Extension at Surgical Margins Are Independent Predictors of Outcomes after Radical Prostatectomy

CE Day, $H$ Djaladat, $M$ Alemozaffar, $M$ Aron, J Cai, $T$ Campanelli, G Lieskovsky, $S$ Daneshmand. Keck Medical Center, University of Southern California, Los Angeles, CA; Norris Comprehensive Cancer Center, University of Southern California, Los Angeles, CA.

Background: A positive surgical margin (PSM) found following radical prostatectomy (RP) is known to affect subsequent recurrence and survival. The extent of PSM, in addition to other pathologic parameters, have also been shown to have a significant impact on clinical outcomes. We examined the effect of length of PSM, extent of disease at PSM, and maximum Gleason score at PSM on outcomes.

Design: Review of 3971 patients undergoing RP at our institution between 1978-2009 revealed 1053 cases with PSM $(26.5 \%), 814$ of which had no neoadjuvant or adjuvant hormone therapy. An initial cohort of 204 cases with measurable tumor at ink were evaluated for: length of PSM (mm), maximum Gleason score at PSM, and maximal extension of PSM (intraprostatic incision (IPI) vs. extraprostatic extension (EPE)). Stepwise multivariable Cox regression models were used to evaluate the impact of the above PSM features as well as age, preoperative PSA, pathologic Gleason score, pathologic stage and adjuvant radiation therapy on biochemical and clinical recurrencefree survival (RFS), and overall survival (OS).

Results: Median follow-up was 14.7 years. The maximum extension of PSM was limited to IPI in $69.1 \%$ and EPE in $30.9 \%$ of patients. The median length of PSM was $4 \mathrm{~mm} ; 85$ with $<3 \mathrm{~mm}, 85$ with $4-12 \mathrm{~mm}$, and 34 with $>13 \mathrm{~mm}$. The maximum GS at PSM was $\leq 6$ in 123 and $>7$ in 81 patients. Ten-year PSA RFS, clinical RFS, and OS were $68.4 \%, 87.3 \%$, and $83.4 \%$, respectively. Multivariable Cox regression modeling showed lengths of PSM $\geq 4 \mathrm{~mm}$ and also $\geq 13 \mathrm{~mm}$ to be independent predictors of PSA and clinical RFS. EPE at PSM independently predicted clinical RFS and OS. Maximum Gleason score at PSM was not independently associated with worse clinical outcomes on multivariable analysis in this cohort.

Multivariable Cox regression modeling
\begin{tabular}{|l|l|l|l||l|l|l||}
\hline \hline & PSA RF & Clinical RFS & Overall Survival \\
\hline \hline & HR & p-value & HR & p-value & HR & p-value \\
\hline \hline PSM Length $>/=4 \mathrm{~mm}$ & 2.703 & 0.0012 & 5.397 & 0.0063 & & \\
\hline PSM Length 4-12mm & 2.406 & 0.0078 & 4.476 & 0.0204 & & \\
\hline PSM Length $>/=13 \mathrm{~mm}$ & 3.413 & 0.0008 & 7.406 & 0.0027 & & \\
\hline EPE at PSM & & & 1.829 & 0.045 & 2.928 & $<0.0001$ \\
\hline
\end{tabular}

Conclusions: Our study shows that PSM lengths at RP of $\geq 4 \mathrm{~mm}$ and $\geq 13 \mathrm{~mm}$ are each independent predictors of biochemical and clinical recurrence. In addition, PSM involving EPE is an independent predictor of clincical RFS and OS. Length in $\mathrm{mm}$ of a PSM and whether the PSM is intra- or extraprostatic should be reported by pathologists, as these findings may aid decision-making regarding adjuvant therapy.

917 Alterations of PTEN Tumor Suppressor Gene in Lethal Prostate Cancer: A Comparative Study Using Chromogenic In Situ Hybridization and Immunohistochemistry

$F$-M Deng, Y Wang, X Wu, WXu, JM Mosquera, M Rubin, J Melamed, M Zhou. New York University Langone Medical Center, New York, NY; Ventana Medical Systems, Inc, Tuscon, AZ; Weill Cornell Medical College, New York, NY.

Background: PTEN is the most frequently deleted tumor suppressor gene in prostate cancer $(\mathrm{PCa})$. Point mutations and epigenetic silencing also contribute to loss of PTEN function. PTEN status, at both gene and protein levels, is a powerful biomarker for PCa. It is often assessed by fluorescence in situ hybridization (FISH) 
and immunohistochemistry (IHC). However, the correlation of FISH and IHC results is not well studied. We compare chromogenic in situ hybridization (CISH) and IHC in determining the PTEN status in lethal PCa.

Design: A tissue microarray consisting of 82 patients with metastatic $\mathrm{PCa}$ was used for simultaneous ISH for PTEN protein and CISH detection of copy number of PTEN and CEN10.

Results: For PTEN CISH, 89 cores (out of 176, 51\%) were informative. 68 (76.4\%), 7 $(7.9 \%)$ and $14(15.7 \%)$ had wild type, hemizygous deletion and homozygous deletion, respectively. PTEN IHC was assessable in 105 cores and was positive, weakly positive and negative in $34(32.4 \%), 48(45.7 \%)$ and $23(21.9 \%)$ cores (Table 1). 70 cores had both PTEN CISH and IHC. 27/28 (96\%) cores positive and 24/28 (86\%) weakly positive for PTEN IHC had wild type PTEN on CISH, while 10/14 (71\%) cores negative for PTEN IHC had PTEN homozygous or hemizygous deletion on CISH ( $\mathrm{P}<0.0001$ by chi square test)

Table 1. Comparison of chromogenic in situ hybridization (CISH) and IHC in determining PTEN status in lethal prostate cancer

status in lethal prostate cancer
\begin{tabular}{|l|l|l||l|l|}
\hline PTEN IHC & PTEN CISH \\
\hline \hline & Homologous deletion & Hemizygous deletion & Wild type & Total \\
\hline Negative & $9(64 \%)$ & $1(7 \%)$ & $4(29 \%)$ & $14(100 \%)$ \\
\hline Weakly positive & $2(7 \%)$ & $2(7 \%)$ & $24(86 \%)$ & $28(100 \%)$ \\
\hline Positive & $0(0 \%)$ & $1(4 \%)$ & $27(96 \%)$ & $28(100 \%)$ \\
\hline
\end{tabular}

$\mathrm{p}<0.0001$ by chi square tes

Conclusions: PCa positive for PTEN IHC have wild type PTEN gene in $96 \%$ cases. $29 \%$ of PCa with loss of PTEN protein expression tested by IHC have wild type PTEN, suggesting mechanisms other than gene deletion including point mutations and epigenetic modification are responsible for silencing PTEN gene in these cases. Clinical evaluation of PTEN gene status requires complimentary methods including IHC and CISH/FISH methodologies.

918 Gleason Score 3+4=7 Prostate Cancer with Minimal Quantity of Gleason Pattern 4 on Needle Biopsy Is Often Associated with Low Risk Tumor

F-M Deng, CC Huang, $M$ Kong, $W X$ Xu, M Zhou, J Melamed. New York University Langone Medical Center, New York, NY.

Background: The 2005 ISUP modified Gleason grading system recommends that any amount of higher grade cancer sampled on needle biopsy should be included in the Gleason score (GS) instead of original grading method of adding together the most common and the second most common Gleason patterns. Some GS 6 cancers by the original Gleason grading system, even with minimal quantity ( $\leq 5 \%)$ of Gleason pattern 4 (GP4) component (GS7miniGP4) in the biopsy, are as the result upgraded to GS7. The evidence supporting this practice is emerging but not well documented. The pathological parameters in the radical prostatectomy (RP) specimens for GS7miniGP4 cases have not been described in the literature.

Design: A total of 256 consecutive needle biopsies with GS 6 or 7 and their corresponding RP specimens collected between 2011 and 2012 at our institute were used in this study. GS was $3+3=6,3+4=7$ and $4+3=7$ in 88,107 and 61 cases. For GS 7 cases, we quantified the Gleason pattern 4 component as the $\%$ of cancer foci. We correlated the pathological parameters of needle biopsies and their corresponding RP specimens obtained at our institute using modified Gleason grading system.

Results: The number of total positive core, cancer length, percentage of cancer of individual core and average cancer percentage over all cores are significantly lower in cases with GS7miniGP4 than those in cases with GP4 6-50\%, respectively. However, there are no significant different between GS7miniGP4 and GS6 groups. The quantity of GP4 in GS7 biopsy significantly correlated with GS, pathological stage and total tumor volume (TV) in the radical prostatectomy specimens. The GS, pathological stage, total TV and insignificant tumor rate in RP is not significantly different between the biopsy groups of GS $3+3=6$ and GS7miniGP4; while all of those parameters are significantly different either between biopsy groups of GS $3+3=6$ and GS $3+4=7$ with GP4 6-50\% or between biopsy groups of GS7miniGP4 and GS7 with GP4 6-50\%. In 22 RP specimens from GS7miniGP4 in the biopsies, 10 cases present with GS6 cancers with pathologically insignificant tumor.

Conclusions: Pathological parameters in the RP are similar between the biopsy groups of GS7miniGP4 and GS6, and the grading of cases with GS7miniGP4 at biopsy are often down-graded in RP specimens. The clinical significance of minimal quantity $(\leq 5 \%)$ of Gleason pattern 4 on biopsies with GS7 PCa need to be further evaluated, particularly because of its potential impact on clinical decisions between active surveillance versus surgery.

\section{PAX 8 Immunostain Pattern of Renal and Extra-Renal Ewing} Sarcomas/PNETs

J Dhillon, T Chen, A Kasprzak, J Johnson, WJ Sexton, M Lloyd, SD Zheng, MB Bui. Moffitt Cancer Center, Tampa, FL; Morsani College of Medicine, USF, Tampa, FL.

Background: PAX 8 is a transcription factor involved in the regulation of organogenesis of the thyroid, kidney, and Mullerian system. It is commonly expressed in epithelial tumors of the thyroid and parathyroid, kidney, thymus, and female genital tract. PAX8 is increasingly used to establish the tissue origin of carcinomas and has recently been identified in PNETs. However, it is unclear if this association is due to renal origin or PNET specific. In this study we investigated the PAX 8 staining pattern of renal and extra-renal Ewing sarcomas/PNETs to explore its potential diagnostic role.

Design: A tissue microarray of 24 cases of extra-renal Ewing/PNETs and 2 separate cases of primary renal PNET whole slide sections were immunohistochemically stained with rabbit polyclonal PAX 8 (dilution 1:5, clone) using an automated stainer. The slides were digitized using an XT whole slide scanner and were scored semi quantitatively by a specialized genitourinary surgical pathologist. The $\mathrm{H}$ score was calculated for each case using a combination of staining intensity and extent as follows: $\mathrm{H}$ score $=1 \mathrm{x} \%$ of tumor cells with weak staining $+2 x \%$ of tumor cells with moderate staining $+3 x$ $\%$ of tumor cells with strong staining, resulting in a total score of $0-300$. The Allred score was calculated using the sum of a proportion score reflecting the percentage of positive staining tumor cells ( 0 , none; $1,1 \square 100 ; 2,1 \square 100$ to $1 \square 10 ; 3,1 \square 10$ to $1 \square 3$; 4 , $1 \square 3$ to $2 \square 3$; and $5,>2 \square 3$ ) and an intensity score representing the average intensity of positive tumor cells ( 0 , none; 1 , weak, 2 , intermediate; and 3 , strong). The proportion and intensity scores are added to obtain a total score, which ranges from 0 to 8 . The positivity cut-off was $\geq 40$ for $\mathrm{H}$ score and $\geq 6$ for Allred score.

Results: PAX 8 was positive in both (100\%) primary renal PNETs and $15(62.5 \%)$ cases of the extra renal PNETs.

$\mathrm{H}$ Score for PAX 8 immunostain

H Score for PAX 8

Renal PNET (2)

Extra-renal PNET (24)

Allred Score for PAX 8 immunostain

Allred Score for PAX 8

Renal PNET (2)

Extra-renal PNET (24)

Conclusions: The association between PAX 8 immunoreactivity and Ewing/PNET was identified in both renal and extra-renal Ewing/PNETs for the first time. Further studies are warranted to verify these findings and to shed light in the tumorigenesis of Ewing/ PNET. However, PAX 8 is not useful in establishment of tissue origin for Ewing/PNET due to its presence in both renal and extra-renal Ewing/PNETs.

920

\section{Cases}

J Dhillon, JG Savell, TJ Kim, AR Giuliano, MA Poch, WJ Sexton, JM Pow-Sang, PE Spiess. Moffitt Cancer Center, Tampa, FL.

Background: Penile carcinoma (ca) accounts for $0.4 \%$ to $0.6 \%$ of all malignancies in men. We report clinicopathological features of 51 invasive penile carcinomas.

Design: 51 cases of invasive penile ca from the surgical pathology files of our Tertiary Cancer Center were analyzed and clinical information was obtained from the institutional clinical files.

Results: Patients ranged in age from 37 to 91 years (mean 63). The ethnic background was - Asian (1), African American (1), Spanish (6) and White (43). The invasive carcinomas were classified as squamous cell ca, NOS (27), warty (9), mixed wartybasaloid (5), papillary, NOS (3), basaloid (4), spindle cell (1), verrucous (1) and basal cell ca (1). Squamous cell ca was further graded as well (7), moderate (15) and poor (5). Histological subtypes presenting at Stage III or IV and the corresponding deceased patients is presented.

Histological subtypes presenting at Stage III or IV and the number of corresponding dead patients

\begin{tabular}{|c|c|c|c|c|c|c|c|}
\hline $\begin{array}{l}\text { Data } \\
\text { Analyzed }\end{array}$ & $\begin{array}{l}\text { Verrucous } \\
\mathrm{Ca}(\mathrm{n}=1)\end{array}$ & $\begin{array}{l}\text { Papillary } \\
\text { Ca, NOS } \\
(\mathrm{n}=3)\end{array}$ & $\begin{array}{l}\text { Warty Ca } \\
(\mathrm{n}=9)\end{array}$ & \begin{tabular}{|l} 
Mixed \\
Warty \\
Basaloid \\
Ca (n=5)
\end{tabular} & $\begin{array}{l}\text { Basaloid } \\
\mathrm{Ca}(\mathrm{n}=4)\end{array}$ & $\begin{array}{l}\text { Spindle } \\
\text { Cell Ca } \\
(\mathrm{n}=1)\end{array}$ & $\begin{array}{l}\text { Squamous Cell } \\
\text { Ca, NOS ( }=27)\end{array}$ \\
\hline $\begin{array}{l}\text { Cases } \\
\text { presenting } \\
\text { at Stage III } \\
\text { or IV }\end{array}$ & $0(0 \%)$ & $1(33.3 \%)$ & $2(22 \%)$ & $4(80 \%)$ & $4(100 \%)$ & $1(100 \%)$ & $10(37 \%)$ \\
\hline $\begin{array}{l}\text { No. of } \\
\text { deceased } \\
\text { Patients }\end{array}$ & $0(0 \%)$ & $3(100 \%)$ & $\begin{array}{l}3 \\
(33.3 \%)\end{array}$ & $3(60 \%)$ & $2(50 \%)$ & $1(100 \%)$ & $15(55 \%)$ \\
\hline
\end{tabular}

Two of the patients were HIV positive. Both of them had HPV related penile ca (warty and basaloid types). HPV ISH was performed in 20 cases.

Results of HPV ISH in 20 cases

\begin{tabular}{|c|c|c|}
\hline Carcinoma Subtype & Positive & Negative \\
\hline Squamous cell ca, NOS (9) & $6(66.6 \%)$ & $3(33.3 \%)$ \\
\hline Warty Ca (3) & $2(66.6 \%)$ & $1(33.3 \%)$ \\
\hline Basaloid Ca (3) & $2(66.5 \%)$ & $1(33.3 \%)$ \\
\hline Papillary Ca (2) & $0(0 \%)$ & $2(100 \%)$ \\
\hline Mixed Warty basaloid $\mathrm{Ca}(2)$ & $2(100 \%)$ & $0(0 \%)$ \\
\hline Verrucous Ca (1) & $0(0 \%)$ & $1(100 \%)$ \\
\hline
\end{tabular}

Clinical follow up available for these 51 patients showed that 23 patients were alive with a follow-up period of 5 to 111 months (mean 52.9)and 28 patients had died 1 to 159 months after diagnosis (mean survival 34.6).

Conclusions: Mixed warty basaloid, basaloid, spindle cell and poorly differentiated squamous cell ca are aggressive subtypes of penile ca. Mixed warty basaloid, basaloid and some of the poorly differentiated squamous cell carcinomas are aggressive subtypes which are HPV related. Additional studies are needed to determine if these subtypes should be uniformly graded as poor and if lymph node dissection should be performed in all cases of mixed warty basaloid, basaloid and spindle cell ca subtypes. HPV related penile carcinomas develop in patients with HIV. These tumors run an aggressive course with a mean survival of 34.6 months.

$921 \quad$ Penile Intraepithelial Neoplasia (PeIN) with and without Associated Invasive Carcinoma - A Study of 71 Cases

J Dhillon, JG Savell, TJ Kim, AR Giuliano, MA Poch, WJ Sexton, JM Pow-Sang, PE Spiess. Moffitt Cancer Center, Tampa, FL.

Background: Penile intraepithelial neoplasia (PeIN) is the corresponding precancerous lesion of the penile carcinoma.

Design: 71 cases of PeINs associated with or without invasive carcinoma were analyzed from the surgical pathology files of our Tertiary Cancer Center and clinical information was obtained from the institutional clinical files. 
Results: The patients ranged in age from 31 to 91 years (mean 62.7). There were 23 cases of PeIN without associated invasive carcinoma all of which were composed of PeIN high grade (HG). These cases were further subclassified as usual type (13), warty (5), basaloid (3) and warty basaloid (2). 48 cases were associated with invasive carcinoma and are further described in Table 1.

\begin{tabular}{|c|c|}
\hline \multicolumn{2}{|c|}{ Squamous cell carcinoma subtype associated with corresponding PeIN } \\
\hline Squamous Cell Carcinoma Subtype $(n=48)$ & \\
\hline Spindle cell $\mathrm{Ca}$ (1) & $\begin{array}{l}\text { Hyperplasia/Low grade (LG), ulcerated } \\
\text { surface }\end{array}$ \\
\hline Verrucous $\mathrm{Ca}(1)$ & Hyperplasia (1) \\
\hline Papillary Ca, NOS (2) & HG, usual type (2) \\
\hline Basaloid Ca (5) & $\begin{array}{l}\mathrm{HG} \text {, basaloid type (4); Hyperplasia, } \\
\text { ulcerated surface (1) }\end{array}$ \\
\hline Mixed warty-basaloid Ca (7) & HG, warty basaloid type (7) \\
\hline Warty $\mathrm{Ca}(8)$ & HG, usual type (3); HG, warty type (5) \\
\hline $\begin{array}{l}\text { Squamous cell ca, NOS (11) [Well Differentiated 2; } \\
\text { Moderately Differentiated 8; Poorly Differentiated 1] }\end{array}$ & HG, usual type (11) \\
\hline $\begin{array}{l}\text { Squamous cell ca, NOS (13) [Well Differentiated 5; } \\
\text { Moderately Differentiated 5; Poorly Differentiated 3] }\end{array}$ & Hyperplasia/LG usual type (13) \\
\hline
\end{tabular}

There were 4 cases of PeIN $(\mathrm{HG}=1$; Hyperplasia/LG $=3$ ) that were associated with lichen sclerosis et atrophicus. These cases also had invasive squamous cell ca, NOS There were 8 cases where HPV ISH was positive in the invasive ca as well as in the PeIN and 11 cases where HPV ISH was negative in the invasive ca as well as in the PeIN. Conclusions: PeIN may be associated with or without invasive ca component. When occurring by itself, PeIN, usual type, is the most common subtype. PeIN histology and the HPV status generally correlate with the histology and the HPV status of the invasive ca component. Some cases of PeIN may show very subtle histologic abnormalities in the form of hyperplasia which may be associated with hyperkeratosis and/or parakeratosis. Lichen sclerosis et atrophicus is rarely associated with PeIN.

$922 \quad$ Looking beyond Morphology: Understanding the Molecula Attributes of the Gleason Grades and Their Biological Significance $Q$ Ding, Z Lichner, C Saleh, S Samaan, P Kupchak, M Dharsee, A Fendler, KR Evans, $K$ Jung, GM Yousef. Keenan Research Centre in the Li Ka Shing Knowledge Institute, St. Michael's Hospital, Toronto, ON, Canada; University of Toronto, Toronto, ON, Canada; MaRS Centre, Toronto, ON, Canada; Free University, Berlin, Germany.

Background: Prostate cancer is the most prevalent cancer in men. The 5-tiern Gleason grading (GG) system is used to assess tumor aggressiveness based on the architectural features. However, it has been reported that there is significant inter-observer variability among pathologists in the assigning of Gleason grades, and the Gleason grade can be higher in resection compared to biopsy. The biological attributes of the GG can help accurate classification based on biology rather than morphology. miRNAs are short, single stranded RNA molecules that function by controlling the expression of their target genes.

Design: miRNAs were extracted from formalin-fixed paraffin embedded tissues, miRNAs profile was examined by TaqMan ${ }^{\circledR}$ Low Density Arrays, miRNA target prediction analyses were performed. miR-29c and miR34a were transfected into prostate cancer cell line, FAK and MAPK signal pathways were analyzed. Cell proliferation rates and migration assay and invasion assay were performed in cell lines.

Results: The expression of 746 miRNAs was screened. 40 miRNAs were found to be significantly differentially expressed between the different Gleason grades. 11 miRNAs were found to be significantly deregulated between Gleason grades 3 and 4. miR-29c was found to be significantly down-regulated between both Gleason grades 3 and 4 as well as Gleason grades 4 and 5. Target prediction of miR-29c and miR-34a showed that these miRs are involved in focal adhesion kinase and MAP-kinase pathways. We validated gene expressions of Col1A1, Src, Prkca, MAPK13 and ITGB before and after miR29c or miR34a transfection. Transfection of miR-29c into PC3 prostate cancer cell line resulted in significant reduction in cell proliferation. Migration and invasion assay were decreased after miR-29c or miR34a transfection.

Conclusions: Our results suggest that miRNAs are involved in prostate cancer progression and promising biomarkers for diagnosis and prognosis.

\section{Targeted Resequencing of High and Low Grade Prostatic} Adenocarcinoma

EJ Duncavage, HJ Abel, PA Humphrey. Washington University, St. Louis, MO.

Background: The genetic differences between low grade (Gleason grade $3+3=6$ or lower) and high grade (Gleason grade $4+4=8$ or higher) are largely unknown. Using a targeted next generation sequencing we sought to determine the frequency of canonical recurrently mutated cancer genes in high and low grade prostate cancer.

Design: We selected a series of 20 radical prostatectomy prostate cancers with high and low grade Gleason scores including $2(2+3), 8(3+3), 1(4+4), 6(4+5), 2(5+4)$, and 1 $(5+5)$ and macro-dissected areas of high tumor cellularity from formalin-fixed blocks. Extracted DNA was then captured using a custom panel of 150 genes implicated in cancer, and sequenced to a mean depth of $847 \times$ (range 450-1030) using $2 \times 101$ bp reads on an Illumina 2500 sequencer. ERG introns 6-10 and TMPRSS2 introns 1-2 plus coding exons were captured. Data were analyzed for a full spectrum of mutations including single nucleotide variants, indels, and translocations. Probable somatic mutations were determined by removing all variants present in the Exome Sequencing or 1000 Genomes Project databases with allele frequencies $>1 \%$; data was further compared to the COSMIC database to determine recurrence across other cancer types. Translocations were detected by ClusterFAST.

Results: TMPRSS2-ERG fusions were found in 3/10 high-grade cases (30\%) and 4/10 $(40 \%)$ low-grade cases; two cases harbored a multi-way rearrangements involving TMPRSS2, $E R G$, and chromosome 7 or 12 . No differences in the breakpoint locations between high and low-grade cancers were observed. In low-grade cancers an average of 9.7 variants predicted to alter protein coding were identified per case of which 1.5 were present in COSMIC, compared to 6.2 average variants of which 1.4 were in COSMIC in high grade cancers. All variants present in COSMIC had a low occurrence ( $<5$ reported cases), except for a single high-grade cancer showed a recurrent TP53 mutation. Previously reported recurrent mutations in prostate cancer including PIK3CA, $C D K N 1 B$, and $P T E N$ were not identified and no recurrent activating mutations were seen. Conclusions: Here we demonstrate that $E R G$-fusions can be identified at the DNA level by targeted sequencing and that mutations in canonical cancer-related oncogenes (ie $K R A S, B R A F, E G F R$ ) are rare in prostate cancer. Outside of one high-grade cancer with a TP53 mutation, there were no genetic differences between low and high-grade prostate cancer.

\section{4} Specimens?

JJ Ebel, KS Pohar, DL Zynger. The Ohio State University Medical Center, Columbus, $\mathrm{OH}$

Background: The lymph node yield during radical cystectomy procedures varies among institutions. Lymph node count is regarded as a controversial surgical quality indicator with uncertain prognostic value. There is a paucity of literature on the factors affecting the lymph node counts determined by grossers and pathologists. We attempted to determine how grossing variables impact lymph node counts in radical cystectomy specimens.

Design: Pathology reports from cystectomies performed for primary bladder cancer from 2007 to 2011 were reviewed. The following were quantified: lymph node count reported by the grosser, lymph node count reported by the pathologist, cases with microscopic evidence of lymph nodes but no nodes identified by the grosser, type of grosser (resident, pathology assistant student, or pathology assistant), and individual grosser. Overcounting and undercounting were considered to occur when the gross assessment of lymph node number differed by 5 or more compared to the microscopic evaluation. Variables were compared to the grosser. Pearson's chi-squared and Fisher's exact test were used to compare groups.

Results: 403 cystectomy reports were reviewed. $21.8 \%$ had overcounting by the grosser and only $1.5 \%$ had undercounting. Uncounted lymph nodes occurred in $10.9 \%$ of cases. There were no significant differences in overcounting by type of grosser $(\mathrm{p}=0.23)$. Residents had significantly more cases with uncounted lymph nodes than pathology assistants $(\mathrm{p}=0.01)$ and approached significance compared to pathology assistant students $(\mathrm{p}=0.06)$. Residents neglected to count lymph nodes in $19.4 \%$ [95\% CI: 13-29], pathology assistants in 8.5\% [95\% CI: 6-13], and students in $8.3 \%$ [95\% CI: 4-18] of cases. Grossers in the fourth quartile of cystectomy grossing volume were found to have fewer cases with uncounted lymph nodes than those in the lower three combined $(\mathrm{p}=0.04)$. For overcounting, there was no difference between the fourth quartile compared to the lower three quartiles $(\mathrm{p}=0.58)$.

Conclusions: Overcounting of lymph nodes by grossers was more frequent than undercounting. Failure to identify lymph nodes occurred more often in resident cases and those with lower cystectomy caseloads. Grosser proficiency is a variable that needs to be considered when comparing interinstitutional data.

925 Metastatic Prostate Adenocarcinoma (PCa.) to the Penis Has an Increased Frequency of Ductal Features: A Series of 28 Cases

C Ellis, JI Epstein. Johns Hopkins Hospital, Baltimore, MD.

Background: Prostate cancer metastatic to the penis is uncommon. There are no large series describing its clinicopathological features.

Design: We identified 28 men with metastatic PCa. to the penis from 1993-2013.

Results: In most cases, the location was noted as "penile urethra" or "penis" with more specific information in 9 cases: bulbomembranous $(n=4)$; corpus $(n=2)$; skin $(n=1)$; foreskin $(\mathrm{n}=1)$; and head of penis $(\mathrm{n}=1)$. The mean age at time of penile metastasis (met.) was 70 years (58-90). 16 had a known prior history of PCa. with a median interval between diagnosis of PCa. and penile met. of 3.5 years ( $0.5-23$ years). 4 men were concurrently diagnosed with PCa. and the penile met. 2 men had no documented prior or concurrent diagnosis of PCa. and 6 had no information about prior history of PCa. but the diagnosis was established on the pathology of the penile met. Morphologic review of the PCa. primary (by report or review of slides) was obtained in 13 cases, where $8 / 13(61.5 \%)$ had ductal features in the primary. Review of all 28 penile mets. showed that $17 / 28(60.7 \%)$ had PCa. with ductal features. Other variant morphologies in the penile mets. were sarcomatoid carcinoma $(n=1)$; small cell carcinoma $(n=2)$; and adenosquamous $(\mathrm{n}=1)$. Extensive angiolymphatic invasion was a common finding in the penile met. IHC stains performed to identify prostatic origin in difficult cases included PSA, PSAP, P501S, PSMA, and NKX3.1 used variably in 22 cases. In 3 cases sent in for consults, the penile met. was misdiagnosed as urothelial carcinoma. Of 17 cases with follow-up information, 5 are alive with relatively short intervals post-penile met. $(6,6,21,27,28$ months $)$ and 1 is alive 116 months later with lung and hilar mets. 10 are dead with a median post-penile met. interval of 24 months (3-109 months).

Conclusions: $\mathrm{PCa}$. involving the penis displays ductal features considerabley more often $(60.7 \%)$ than $\mathrm{PCa}$. in general $(8-13 \%)$. The exact mechanism is unclear, and spread via implantation of cells from the urethra is possible along with lymphovascular spread. Features that can cause difficulty in recognizing these tumors' prostatic origin include variant morphology, and often a long interval from the primary or no prior documented primary. IHC should be done to rule out PCa. in penile urethral tumors with morphology different from typical squamous or urothelial carcinoma. Although most patients with penile mets. are dead of disease within a couple of years, there are a few long-term survivors on hormonal therapy. 
926 Rb Loss Is Characteristic of Prostatic Small Cell Neuroendocrine Carcinoma but Rare in Primary and Metastatic Adenocarcinomas

C Ellis, H-L Tan, J Hicks, G Netto, J Epstein, W Liu, WB Isaacs, AM De Marzo, TL Lotan. Johns Hopkins Medical Institutions, Baltimore, MD; Wake Forest School of Medicine, Winston-Salem, NC

Background: Neuroendocrine (NE) carcinoma of the prostate is anticipated to become increasingly common with advances in pharmacologic androgen suppression. Recently, we showed that $\mathrm{Rb}$ protein loss occurs in close to $90 \%$ of small cell NE carcinomas of the prostate, with $R B I$ allelic loss occurring in a similar fraction, suggesting that $\mathrm{Rb}$ loss may be associated with of NE differentiation in prostate tumors. Here, we assessed the frequency of $\mathrm{Rb}$ loss in a spectrum of primary and metastatic non-small cell prostatic carcinomas with and without NE features.

Design: We used a genetically validated immunohistochemistry assay to assess $\mathrm{Rb}$ status in 150 predominantly high grade prostatic adenocarcinomas $(20.6 \%$ Gleason score 7 and $57 \%$ Gleason score $8-10$ ), 35 primary non-small cell prostatic carcinomas annotated as having NE differentiation (including 6 large cell NE carcinomas and 29 adenocarcinomas with morphologic or significant immunophenotypic evidence of NE differentiation) and 14 castrate resistant metastatic non-small cell carcinomas.

Results: Only 6.7\% (10/150) of primary high grade prostatic adenocarcinomas showed $\mathrm{Rb}$ loss. Of primary non-small cell carcinomas with neuroendocrine differentiation, $11 \%$ (4/35) showed $\mathrm{Rb}$ loss, including $10.3 \%$ (3/29) of cases with morphologic or immunophenotypic evidence of NE differentiation and $16.7 \%(1 / 6)$ of large cell NE carcinomas. $21 \%(3 / 14)$ of metastatic castrate resistant adenocarcinomas showed $\mathrm{Rb}$ protein loss, including one case with diffuse chromogranin/synaptophysin positivity. Conclusions: In stark contrast to small cell carcinomas, Rb protein loss occurs in only a small minority of high grade primary prostatic adenocarcinomas, and is infrequently seen in non-small cell carcinomas with morphologic or immunophenotypic evidence of neuroendocrine differentiation. $\mathrm{Rb}$ loss is slightly enriched in castrate resistant prostate tumors, suggesting that complete genetic inactivation of $R B 1$ occurs predominantly late in prostatic adenocarcinoma progression. In poorly differentiated primary prostatic tumors, $\mathrm{Rb}$ loss as assessed by immunohistochemistry may be an additional useful marker of small cell differentiation.

927 Enhancer of Zeste Homolog 2 (EZH2) Over-Expression Is Associated with Metastasis but Not with Local Tumor Characteristics nor with Mortality in Urothelial Carcinoma of the Bladder

HElMansi, A Aprikian, S Tanguay, B Bachir, L Florianova, W Kassouf, F Brimo. McGill University Health Center, Montreal, QC, Canada.

Background: Enhancer of Zester Homolog 2 (EZH2), a histone methyltransferase mediating chromatin condensation and epigenetic modulation has been shown to be over-expressed in various human carcinomas and to be associated with adverse clinico-pathological characteristics and biologic behavior. The expression of EZH2 in urothelial carcinoma (UCA) is poorly characterized with conflicting results from a limited number of studies.

Design: Using eight tissue microarrays obtained from cystectomy specimens, EZH2 immnoexpression was evaluated in 160 invasive UCA (368 cores) with 49 matched metastasis, as well as 55 cases of carcinoma in-situ (CIS), 25 high-grade papillary UCA (PAP) and 105 cores of benign urothelium. Expression of the marker was recorded using $\mathrm{H}$-score (HS) (staining intensity $\mathrm{X} \%$ of positive cells).

Results: $77 \%$ of patients were male and the mean age was 70 years. In comparison to benign urothelium $(\mathrm{H}$-score $=13.8)$, there was a significant increase in $\mathrm{EZH} 2$ expression in CIS (HS=92), PAP (HS=91), and invasive UCA (HS=90) ( $\mathrm{p}=0.0001)$. Metastases were associated with a significantly higher HS than invasive UCA overall and in the matched cases as well ( $110 \mathrm{vs} 73, \mathrm{p}=0.005)$. Correlation of $\mathrm{EZH} 2$ expression with the cystectomy parameters did not reveal any significant association with $\mathrm{pT}$ stage $(\mathrm{p}=0.4)$, $\mathrm{pN}$ stage $(\mathrm{p}=0.5)$, lymphovascular invasion $(\mathrm{p}=0.5)$, nor margin status $(\mathrm{p}=0.8)$. On univariate analysis, recurrence was associated with $\mathrm{pN}$ stage $(\mathrm{p}=0.05)$, lymphovascular invasion $(\mathrm{p}=0.05)$ and low HS values $(\mathrm{p}=0.05)$, and the latter preserved its significant association on multivariate analysis $(\mathrm{p}=0.02)$. On another hand, death was significantly associated with $\mathrm{pN}$ stage $(\mathrm{p}=0.05)$ and lymphovascular invasion $(\mathrm{p}=0.03)$ but not with EZH2 levels $(\mathrm{p}=0.7)$.

Conclusions: EZH2 is over-expressed in UCA in comparison to benign urothelium and is significantly associated with metastasis, indicating its key role in driving oncogenesis. However, its over-expression does not seem to correlate with local tumor characteristics nor with overall clinical outcome.

928 Prostate Biopsies with Discontinuous Cancer Involvement: Gap or No Gap?

$O$ Eze, $W X u, F-M$ Deng, J Melamed, $M$ Zhou. New York University Langone Medical Center, New York, NY.

Background: Prostate cancer (PCa) extent in prostate biopsies (PBx) is an important prognostic parameter and is used to choose treatment modalities. It is not uncommon to see $\mathrm{PBx}$ with discontinuous cancer involvement with benign tissue separating cancer foci (PBx-gap). How to measure the tumor extent in such biopsies is controversial. We studied how discontinuous cancer involvement affected various volume measurement parameters and potentially impacted the patient selection criteria for active surveillance. Design: PBx-gap was identified in authors' surgical pathology file. Any gap $\geq 1 \mathrm{~mm}$ was considered discontinuous involvement. Tumor linear length and $\%$ of core involvement including and excluding the gap were measured. For each case, the total tumor linear length and mean $\%$ of biopsy core involvement, the greatest tumor length and greatest $\%$ core involvement in any single core were also calculated when including and excluding the gap. The total tumor volume (TV), \% of prostate involved by $\mathrm{PCa}(\mathrm{PCa} \% \mathrm{RP})$ and TV of dominant tumor nodule (DN) in radical prostatectomy (RP) were calculated with a grid method.

Results: 67 PBx-gap from 47 cases were included. The mean gap length was 3.2 (range $1-9) \mathrm{mm}$. In these biopsy cores, the gap was longer in GS6 (3.8 $\pm 2.4 \mathrm{~mm})$ than in GS $\geq 7$ cases $(2.6 \pm 1.3 \mathrm{~mm}, \mathrm{p}=0.033)$. On case level, the greatest tumor length and $\%$ core involvement in any single core were $8.8 \mathrm{~mm}$ and $70.0 \%$ when including the gap, and $7.3 \mathrm{~mm}$ and $58.0 \%$ when excluding the gap ( $\mathrm{p}<0.001$ for both). However, the greatest $\%$ core involvement in any single core correlated with $\mathrm{PCa} \% \mathrm{RP}$ only when the gap was excluded $(\mathrm{p}=0.023)$. In a linear regression model, the greatest tumor length in any single core significantly correlated with the TV of DN in RP when the gap was excluded. Conclusions: When PBx is discontinuously involved by $\mathrm{PCa}$, the gap is significantly greater in GS6 than GS7 cases. Estimation of tumor extent when excluding the gap correlates significantly better with overall tumor \% in RP and TV of the dominant tumor nodule. Our finding suggests that gap should be excluded when estimate the tumor extent in PBx with discontinuous cancer involvement.

\section{ERG, SPINK1 and AR Expression in Prostatic Adenocarcinoma in} Men 45 Years Old or Younger Treated by Radical Prostatectomy

SM Falzarano, K Streator Smith, P Carver, C Magi-Galluzzi. Cleveland Clinic, Cleveland, $\mathrm{OH}$

Background: ERG is the most common recurrent chromosomal rearrangement in prostate cancer (PCA). ETS gene fusions have been implicated to play a critical role in $\sim 50 \%$ of PSA-screened PCA surgical cohorts, leaving the genetic aberration in the remaining cases largely unknown. SPINK1 (serine peptidase inhibitor, Kazal type 1) is over-expressed in a sub-set of PCA. In this study we examined the expression of these proteins in a large cohort of young PCA patients.

Design: Men 45 years old or younger diagnosed with PCA from 2000 to 2013 and treated with radical prostatectomy (RP) were included in the study. Preoperative [age, PSA, positive digital rectal examination ( $+\mathrm{DRE})$, race, family history $(\mathrm{FH})]$ and postoperative characteristics [Gleason score (GS), pathologic stage (pT), tumor volume (TV)] were evaluated in addition to ERG, SPINK1 and androgen receptor (AR) protein expression. Results: $139(2.7 \%)$ men were age 45 or younger. Median age and preoperative PSA was 44 years, and $4.5 \mathrm{ng} / \mathrm{ml}$, respectively. DRE was positive in $15 \%$. Race was as follows: $75 \%$ Whites, $20 \%$ Blacks, $2 \%$ Hispanics, and 3\% unknown. Positive FH was present in 63 (45\%) men: $43 \%$ Whites, $50 \%$ Blacks, $67 \%$ Hispanic, and $50 \%$ of unknown race. RP GS was 6 in $38 \%, 7$ in $56 \%, 7$ with tertiary 5 in $2 \%,>8$ in $3 \%$; pathologic stage was T2 in $82 \%$ and $\mathrm{T} 3$ in $18 \%$. Tumor volume was low in $22 \%$, medium in $56 \%$, and extensive in $22 \%$ of cases. ERG was detected in $81 / 133(61 \%)$ cases; SPINK1 in $21 / 120(18 \%)$ cases, $17(81 \%)$ of which were ERG negative; AR was weakly positive in 37/119 (31\%) cases, moderately in $32 \%$, strongly positive in $31 \%$, and negative in $6 \%$. There was no correlation between presence of ERG or SPINK1, and AR positivity. There was no difference in age, PSA, +DRE, and FH between ERG or SPINK1 positive and negative cases. However, Whites were more likely to have ERG-positive or SPINK1-positive tumors than Blacks ( $\mathrm{p}=0.0003$ and $\mathrm{p}=0.0171$, respectively). There was no association between AR and age, PSA, +DRE, or race. However, AR moderate/strong expression was significantly associated with positive $\mathrm{FH}(\mathrm{p}=0.03)$. No association was seen between presence or absence of ERG, SPINK1 or AR expression and RP GS, pT, and TV.

Conclusions: ERG and SPINK1 detection was not significantly associated with age, PSA, +DRE, FH or postoperative characteristics. Higher incidence of ERG and SPINK1 expression in Whites compared to Blacks further underline the importance of cohort selection. The association between AR expression and positive FH suggests a genetic predisposition to high androgen stimulation.

930 IGF1R Immunoexpression in Superficial Non-Muscle Invasive Urothelial Carcinoma of Urinary Bladder

SF Faraj, N Gonzalez-Roibon, SM Bezerra, E Munari, R Sharma, TJ Bivalacqua, MP Schoenberg, GJ Netto, K Rezaei. Johns Hopkins Medical Institutions, Baltimore, MD; George Washigton University, Washington, DC.

Background: Insulin-like growth factor-1 receptor (IGF1R) is a transmembrane tyrosine kinase receptor involved in cell proliferation and differentiation. IGF1R is overexpressed in several tumors and is currently under investigation as a potential target of therapy. Previously we demonstrated IGF1R overexpression in a cystectomy cohort with a potential impact on prognosis. In the current study we explore IGF1R expression in transurethral resection of bladder (TURB) specimens of non-muscle invasive bladder cancer (NMI-BC).

Design: A total of five tissue microarrays (TMA) were constructed. Two of the TMAs included 77 TURB specimens from Johns Hopkins Hospital (1971 to 1995) and three included 54 TURB specimens from George Washington University (1998-2008). Formalin-fixed paraffin-embedded paired tumor and benign samples were spotted three to four times each. Immunohistochemistry was performed for IGF1R (G11, Ventana Medical Systems) and a membranous staining was evaluated. A scoring method analogous to that for HER2 expression in breast carcinoma was used and the highest score was assigned in each tumor.

Results: IGF1R was expressed in 88 of 100 NMI-BC. IGF1R score 1+ was demonstrated in $39 \%, 2+$ in $28 \%$ and $3+$ in $21 \%$. There was no difference between tumor and paired benign urothelium $(\mathrm{p}=1)$. Tumor recurrence-free survival and tumor progression-free survival (PFS) rates were $43.2 \%$ and $81.8 \%$, respectively. Higher levels of IGF1R expression were correlated with a favorable tumor recurrence-free survival [OR: 0.58 , $\mathrm{p}=0.021]$. There was no association between IGF1R expression and PFS [OR: 0.69, $\mathrm{p}=0.18]$. On multivariate analyses, higher levels of IGF1R expression remained a predictor of favorable tumor recurrence-free survival when adjusting for demographic and pathologic features such as age [OR: $0.58, \mathrm{p}=0.023$ ], gender [OR: $0.59, \mathrm{p}=0.031$ ], pT category [OR: $0.59, \mathrm{p}=0.034]$ and grade [OR: $0.57, \mathrm{p}=0.022$ ]. 
Conclusions: IGF1R is expressed in a high frequency in NMI-BC. In contrast to our previous findings in muscle-invasive bladder cancer, higher levels of IGF1R expression in TURB tissue appears to be associated with a better outcome in NMI-BC.

931 GATA3 Expression in Sarcomatoid Urothelial Carcinoma of the Bladder

N Fatima, AO Osunkoya. Emory University School of Medicine, Atlanta, GA.

Background: GATA3 (GATA binding protein 3) is a member of the GATA family of 6 zinc finger transcription factors. GATA3 has been shown to be a novel marker for urothelial carcinoma. Sarcomatoid urothelial carcinoma is a dedifferentiated, rare and aggressive biphasic variant of urothelial carcinoma that exhibits morphological and immunohistochemical evidence of epithelial and mesenchymal differentiation. The current available data on GATA3 expression in sarcomatoid urothelial carcinoma is limited, especially in the non-TMA based setting. In this study, we analyzed the expression of GATA3 in sarcomatoid urothelial carcinoma of the bladder in cystectomy/ cystoprostatectomy specimens.

Design: A search was made through our surgical pathology and consultation files for cystectomy/cystoprostatectomy specimens with a diagnosis of sarcomatoid urothelial carcinoma. All cases were re-reviewed by a Urologic Pathologist. Only cases with available tissue blocks were selected. Immunohistochemical staining for GATA3 was performed on selected blocks with sarcomatoid urothelial carcinoma, and staining in adjacent/overlying conventional urothelial carcinoma and/or benign urothelium was also documented.

Results: Twenty two cases were obtained. Sixteen of 22 (73\%) cases of sarcomatoid urothelial carcinoma were positive for GATA3 (nuclear staining). In the 7/22 (27\%) cases that were negative for GATA3, it was observed that these cases were predominantly composed either of pleomorphic undifferentiated sarcomatoid areas, or foci composed of extensive heterologous elements (chondroid, osteoid or rhabdoid). GATA3 staining was positive in the adjacent/overlying conventional urothelial carcinoma and/or benign urothelium in all cases.

Conclusions: This is one of the largest studies to date analyzing the expression of GATA3 in sarcomatoid urothelial carcinoma in cystectomy/cystoprostatectomy specimens. GATA3 is expressed in the majority of cases of sarcomatoid urothelial carcinoma. Negative expression may however be observed in cases composed predominantly of pleomorphic undifferentiated sarcomatoid areas or extensive heterologous elements. We recommend including GATA3 in the panel of immunohistochemical stains for metastatic sarcomatoid carcinomas of unknown origin, especially if a bladder primary is being considered in the differential diagnosis.

932 Sarcomatoid Urothelial Carcinoma of the Bladder: A Clinicopathologic Analysis of $\mathbf{3 6}$ Cases

N Fatima, AO Osunkoya. Emory University School of Medicine, Atlanta, GA

Background: Sarcomatoid urothelial carcinoma (SARCUCA) is a dedifferentiated biphasic tumor that exhibits morphological and/or immunohistochemical evidence of epithelial and mesenchymal differentiation. In this series, one of the largest to date, we analyzed the clinicopathologic features of this rare variant of UCA.

Design: A search was made through our surgical pathology files, and consultation files of the senior author for cases of SARCUCA from 2005-2013. All the slides were retrieved and re-reviewed, and clinical data was also obtained including follow up.

Results: Thirty six cases of SARCUCA were identified. Mean patient age was 71 years (range: 51 to 88 years). Twenty five of $36(69 \%)$ patients were male and 11/36 (31\%) patients were female. Twenty five cases were from cystectomy/cystoprostatectomy specimens, 7 cases from transurethral resection of bladder tumor (TURBT) specimens and 4 cases were from biopsy specimens. The mean tumor size was $5 \mathrm{~cm}$ (range: 1.4 to $13.0 \mathrm{~cm}) .3 / 36(8 \%)$ cases had focal heterologous components ( 1 case both chondroid and osteoid, 1 case chondroid and 1 case rhabdoid). Other variants of UCA present were; squamous differentiation $7 / 36(19 \%)$ cases, glandular differentiation $4 / 36(11 \%)$ cases, plasmacytoid differentiation $2 / 36(6 \%)$ cases and $1 / 36(3 \%)$ case each of micropapillary and lymphoepithelioma-like differentiation. In the cystectomy/cystoprostatectomy cases, the trigone was the most frequently involved area (13/25, $52 \%$ cases) followed by lateral and posterior walls $(10 / 25,40 \%$ and $8 / 25,32 \%$, respectively), with some tumors being multifocal. Fifteen of $25(60 \%)$ cystectomy/cystoprostatectomy cases were pT3a $(3 \mathrm{pN} 2,3 \mathrm{pN} 1,8 \mathrm{pN} 0,1 \mathrm{pNX}), 7 / 25(28 \%)$ cases were pT4a $(2 \mathrm{pN} 2,3$ pN1, 2 pN0) and $3 / 25(12 \%)$ cases were pT2b (1 pN1, 2 pN0). Thirteen of $30(43 \%)$ cases with a surface component had associated overlying urothelial carcinoma in situ. Two of 7 TURBT (28\%) specimens and 1 of 4 biopsy (25\%) specimens had invasion into muscularis propria (Detrusor muscle). Five of 7 (71\%) TURBT and 3 of $4(75 \%)$ biopsy specimens had invasion into lamina propria. All patients with available history presented with hematuria. Twenty one of $29(72 \%)$ patients with available social history had a significant history of smoking. Twenty one of $36(58 \%)$ patients died within a year of presentation.

Conclusions: SARCUCA is more prevalent in males, with the mean age of 71 years in our series. Smoking is an important risk factor. SARCUCA is an aggressive variant of UCA which commonly presents at an advanced stage, and over $50 \%$ of patients in our series died of disease within one year of presentation.

933 Predictive Value of Nuclear Grade for Peritumoral Pseudocapsule Penetration in Subtypes of Small Renal Cell Carcinoma - Appraisal of 161 Tumors

J Feng, L Wang, H Alvarez, H Chen, GN Gupta, MM Picken. Loyola University Medical Center, Chicago, IL.

Background: Renal tumors are frequently surrounded by a thin rim of fibrous tissue forming a pseudocapsule and its integrity is important in the nephron-sparing management of small renal cell carcinomas (RCC). However, the integrity of the pseudocapsule may be compromised by partial or complete pseudocapsule penetration (PSP) and evaluation of the extent of this PSP may be limited in conservative surgeries, such as enucleation. This study sought to examine nuclear tumor grade as a possible risk factor for PSP.

Design: 161 small $(\leq 4.0 \mathrm{~cm}) \mathrm{RCC}$, resected by total or partial nephrectomy, or tumor enucleation, were investigated and the tumor pseudocapsule was evaluated for its integrity. Tumors were classified into 2 groups: either low or high grade (nuclear grades 1-2 and 3-4, respectively). The unpaired Student $t$ test and $\mathrm{X}^{2}$ test were used to evaluate possible statistical interrelationships between nuclear grade variables and the risk of PSP. Results: There were 109 low \& 52 high grade tumors. In the former group, 24.5\% (24 cases) demonstrated PSP, while 50.9\% (28 cases) in the high nuclear-grade group had focal PSP. The percentage of PSP in the high nuclear-grade group was significantly higher than that in the low-grade group $(\mathrm{p}<0.01)$. The $\mathrm{X}^{2}$ test was also performed for different subtypes of RCC. Interestingly, only in the clear cell RCC group a significant difference in PSP percentage between these two groups was determined $(\mathrm{P}<0.01)$ (Figure 1), while there was no statistically significant difference in either the papillary or chromophobe carcinoma groups ( $\mathrm{p}=0.61$ and $\mathrm{p}=0.09$, respectively) (Figure 2).

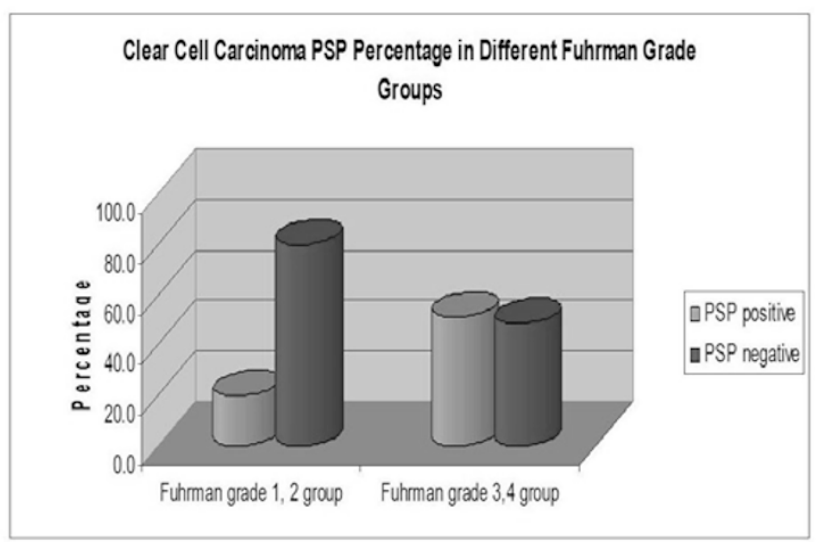

Figure 1

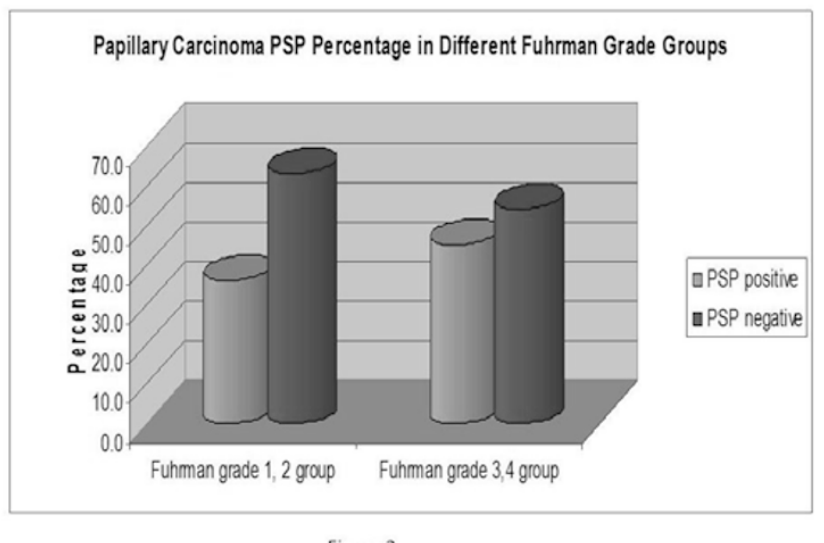

Figure 2

Conclusions: In this study, in clear cell RCC, high nuclear tumor grade was associated with a significantly higher risk of PSP. No such association was seen in papillary and chromophobe RCC. Thus, high nuclear grade clear cell RCC may require a more diligent evaluation of the surgical margin.

934 Gleason Score 7 with Tertiary Pattern 5: A Contemporary Analysis SW Fine, J Chang, JB Poon, DD Sjoberg, HA Al-Ahmadie, Y-B Chen, A Gopalan, SK Tickoo, VE Reuter. Memorial Sloan-Kettering Cancer Center, New York, NY.

Background: Although tertiary Gleason patterns (TP) have been widely mentioned in the literature during the past decade, there is still controversy over their prognostic significance in various Gleason scores (GS) and TP reporting practices. In this study, we focus on the pathologic and biochemical recurrence (BCR)-free outcomes of patients with GS 7 and TP5.

Design: We identified a cohort of 1513 whole-mounted and entirely-submitted radical prostatectomies (2006-2012) with final GS 7-9. Specimens were reviewed for extraprostatic extension (EPE), seminal vesicle (SVI) and lymph node invasion (LNI) and positive surgical margins ( $+\mathrm{SM})$. Patients were divided into 2 groups and comparisons were performed within each group using Fisher's exact test. Group 1: 3+4, 3+4 with TP5 and 4+4; Group 2: 4+3, 4+3 with TP5, 4+4 and 4+5. BCR-free survival probabilities were estimated using Kaplan-Meier method; groups were also compared using log rank test and proportional hazards Cox model, adjusted for EPE/ $\mathrm{SVI} / \mathrm{LNI} /+\mathrm{SM}$, to test for differences in BCR-free survival. Median follow-up for BCR-free survivors was 3.96 years. 
Results: Group 1: Patients with GS 3+4 with TP5 [n=29] were more likely to develop EPE \& +SM compared to GS $3+4$ patients [ $\mathrm{n}=1009$ ] (EPE: $52 \%$ v $26 \%$, $\mathrm{p}=0.005$; +SM: $28 \%$ v $13 \%, p=0.045$ ). Compared to $3+4$ with TP5 patients, those with GS $4+4$ [n=24] had significantly different BCR-free survival probabilities on both log rank test and Cox model ( $\mathrm{p}=0.006$ and $\mathrm{p}=0.042$ ), while those with GS $3+4$ had significantly different BCR-free survival on $\log$ rank test $(\mathrm{p}=0.047)$ but not when adjusted for pathologic variables with the Cox model $(\mathrm{p}=0.3)$. Group 2: Patients with $4+3$ with TP5 [ $\mathrm{n}=91]$ were significantly more likely to develop SVI, LNI \& EPE compared to GS 4+3 patients [n=291] (SVI: $25 \%$ v $12 \%, p=0.004$; LNI: $29 \%$ v $12 \%, p=0.0005$; EPE: $75 \%$ v $62 \%$, $\mathrm{p}=0.032$ ) and $\mathrm{BCR}$-free survival probabilities were also significantly different (log rank test: $\mathrm{p}=0.001$ and Cox model: $\mathrm{p}=0.016$ ). At the same time, patients with GS $4+3$ with TP5 were significantly less likely to develop LNI compared to $4+5$ patients [ $\mathrm{n}=69]$ ] $(29 \%$ vs $46 \%, \mathrm{p}=0.03$ ) and BCR-free survival differences between $4+3$ with TP5 and $4+5$ patients were significant in both $\log$ rank and Cox model $(\mathrm{p}<0.0001$ and $\mathrm{p}=0.001)$. No difference was observed when comparing GS $4+3$ with TP5 patients to $4+4$ patients. Conclusions: In this cohort, TP5 impacted some pathologic, but not adjusted BCR-free outcomes for GS $3+4=7$ patients. In contrast, both pathologic and adjusted BCR-free outcomes for GS $4+3=7$ with TP5 are intermediate between $4+3$ and $4+5$ patients. Reporting TP5 in GS 4+3 has clinicopathologic implications.

\section{Clinicopathologic Implications of the Diagnosis of Atypical Small} Acinar Proliferation (ASAP) in the Era of Active Surveillance

SW Fine, HA Al-Ahmadie, Y-B Chen, A Gopalan, SK Tickoo, VE Reuter. Memorial Sloan-Kettering Cancer Center, New York, NY.

Background: The association between ASAP on prostatic needle biopsy (NB) and cancer on subsequent NB is well known, with $\sim 40 \%$ cancer detection rate. In this era of active surveillance, an increasing number of patients with low grade/low volume disease $(\mathrm{LG} / \mathrm{LV})$ are being managed by active surveillance rather than receiving radical intervention. However, it is not known how many cancers detected after an ASAP diagnosis are actually of LG/LV. In this study, follow-up NB and radical prostatectomy (RP) results in a subset of patients with an ASAP diagnosis on their initial NB at one institution were examined for Gleason score (GS) and quantity of prostate cancer detected.

Design: We identified 200 patients with an ASAP diagnosis on their first NB at our institution, of which 89 had at least one follow-up NB. 55/89 had a prior NB outside our institution, 45 with cancer (which were excluded). For the remaining 44 patients, we determined prior diagnosis to ASAP (in 10/44), \# of follow up NB, pathologic findings in each NB and highest grade/quantity of cancer detected. 11/44 subsequently underwent RP and those pathologic results were also reviewed.

Results: Findings on outside NB prior to ASAP [n=10 patients] were: benign (7), ASAP (2) and PIN (1). Of 44 patients, $29(66 \%)$ has 1 follow-up NB, $9(20 \%)$ had 2 and $6(14 \%)$ had $>2$ (range: $3-6) .20 / 44(45 \%)$ patients had cancer on their first repeat $\mathrm{NB}, 15$ with $\mathrm{LG} / \mathrm{LV}$. In $36 / 44$ (82\%) patients, the repeat NB revealed findings ranging from benign to $L G / L V$, including: benign [ $\mathrm{n}=12,27 \%$ ], ASAP [ $\mathrm{n}=6,14 \%]$, PIN [ $\mathrm{n}=2$, $5 \%$ ] or $\mathrm{LG} / \mathrm{LV}$ (GS $6, \leq 3$ positive cores, $\leq 10 \%$ involvement) [ $\mathrm{n}=16,36 \%$ ]. $4 / 16$ with $\mathrm{LG} / \mathrm{LV}$ findings had RP that revealed GS 6 , organ-confined disease $[\mathrm{n}=3]$ or GS $3+4=7$ with focal extraprostatic extension in a dominant anterior tumor $[\mathrm{n}=1]$. In the other 8 $(18 \%)$ the subsequent NB was indicative of clinically significant cancer, including: GS $3+4=7[\mathrm{n}=7,16 \% ; 1-3$ positive cores, $3-40 \%$ involvement] or GS $4+4=8[\mathrm{n}=1,2 \% ; 1$ positive core, $10 \%$ involvement]. $7 / 8$ patients with $\mathrm{GS} \geq 7$ underwent $\mathrm{RP}$, all showing dominant anterior tumors with GS $3+4=7[n=4]$ or $4+3=7[n=3], 5$ organ-confined and 2 with anterior extraprostatic extension.

Conclusions: The majority of patients with ASAP studied show no cancer or active surveillance-eligible disease, which may have implications for the work-up of atypical foci. The finding that all patients who showed GS $\geq 7$ cancer had dominant anterior tumors at RP indicates that increased attention to the anterior prostate should be a routine part of the management of patients with an ASAP diagnosis.

936 Response Rates and Survival Prediction in Metastasizing Bladder Cancer after Neoadjuvant Chemotherapy

A Fleischmann, A Perren, GN Thalmann, R Seiler. University of Bern, Bern, Switzerland. Background: Neoadjuvant chemotherapy (NCT) before surgery has been shown to improve survival of bladder cancer patients. The histological effects of this therapy on primary tumors and corresponding metastases have never been compared and the best prognosticator for these cancers still has to be determined.

Design: A cohort of 42 patients with lymph node positive urothelial bladder cancers underwent NCT (median 4 cycles) before cystectomy and lymphadenectomy. Tumor regression grades (TRG) were defined histologically: TRG 1: no residual cancer cells, TRG 2: residual cancer cells occupying less than $50 \%$ and TRG 3:50\% or more of the fibrotic tumor bed. Separate TRG were assigned to the primary tumor and to the metastasizing component. Various parameters including the higher ("dominant") of both TRG per patient and the $\mathrm{ypT}$ and $\mathrm{ypN}$ stage groups according to the $7^{\text {th }}$ TNM classification were tested for stratification of overall survival (OS)

Results: TRG 1,2 and 3 was observed in $n=8, n=15$ and $n=19$ of the primary tumors and in $\mathrm{n}=17, \mathrm{n}=9$ and $\mathrm{n}=16$ of the corresponding metastasizing tumor components. Dominant TRG 1,2 and 3 was assigned to $n=7, n=13$ and $n=22$ patients; stage group 0 , I/II, III and IV was assigned to $\mathrm{n}=7, \mathrm{n}=6, \mathrm{n}=4$ and $\mathrm{n}=25$ patients. In univariate analysis, all different TRG (primary tumor, lymph node metastases, pooled), ypT, ypN and the stage groups stratified OS significantly $(\mathrm{p}<0.05)$. In multivariate analysis, the dominant TRG was the only independent risk factor $(\mathrm{p}<0.05)$.

Conclusions: Lymph node metastases from bladder cancers show a trend for stronger tumor regression after NCT than the primary tumors. OS stratification according to the dominant TRG per patient is superior to all other tested parameters including the stage groups defined in the TNM classification. These data might help (a) to explain the beneficial role of NCT reported in the BA06 30894 trail and (b) to enhance prognostication in these patients.

937 RNA Binding Motif Protein 3 (RBM3) Over-Expression Is Associated with Favorable Histopathological Findings but Not with Outcome in Urothelial Carcinoma of the Bladder

L Florianova, B Bachir, H Elmansy, S Tanguay, A Aprikian, W Kassouf, F Brimo. McGill University Health Center, Montreal, QC, Canada.

Background: RNA binding motif protein 3 (RBM3) is a single-strand RNA-binding protein involved in RNA metabolism and the regulation of gene transcription in cell survival under adverse growth conditions. Although it has initially been suggested to have proto-oncogenic role, a limited number of studies have instead shown high expression of RBM3 to be associated with increased survival in several cancer types. The present study analyzes the putative prognostic role of RBM3 in a large cohort of UCA. Design: Using tissue microarrays obtained from cystectomy specimens, RBM3 immnoexpression was evaluated in 160 invasive UCA (368 cores) with 49 matched metastases, as well as 55 cases of carcinoma in-situ (CIS), 25 high-grade papillary UCA (PAP) and 105 benign urothelium. Immunoexpression was recorded using H-score (HS) (staining intensity $\mathrm{X} \%$ of positive cells).

Results: Among the non-invasive lesions, CIS showed the highest HS (mean=140) followed by benign urothelium (mean=97) and PAP (mean=64). Metastases were associated with a significantly higher HS than primary invasive UCA (mean of 26 and 96 for matched primary and metastatic cases respectively; $\mathrm{p} \leq 0.0001$ ). Of the cystectomy pathological parameters, RBM3 over-expression was significantly associated with a lower $\mathrm{pT}$ stage $(\mathrm{p} \leq 0.0001)$ and showed a trend toward the absence of lymphovascular invasion $(\mathrm{p}=0.09)$, while no association was found with $\mathrm{pN}$ stage $(\mathrm{p}=0.35)$ nor surgical margin status $(\mathrm{p}=0.81)$. Recurrence analysis: On univariate analsysis, $\mathrm{pN}$ stage and lymphovascular invasion were strongly associated with recurrence $(\mathrm{p}=0.005$ and 0.03 respectively). In comparison, $\mathrm{pT}$ stage showed trends toward significant association $(\mathrm{p}=0.1)$ while $\mathrm{HS}$ and surgical margins status showed none $(\mathrm{p}=0.49$ and 0.97 respectively). Multivariate analysis (MVA) confirmed the lack of association between HS and recurrence $(\mathrm{p}=0.75)$. Mortality analysis: On univariate analysis, $\mathrm{pT}$ stage was strongly associated with death $(\mathrm{p}=0.01)$ while $\mathrm{pN}$ stage, lymphovascular invasion, surgical margin status and HS were not. The lack of association between HS and death was maintained using a MVA model $(\mathrm{p}=0.32)$.

Conclusions: Stronger RBM3 immunoexpression correlates with favorable tumor histopathological characteristics at cystectomy. However, RBM3 does not seem to carry a clinical prognostic significance. The role of RBM3 in UCA and whether it can definitely be ascribed to the overall clinical course of the disease is yet to be determined.

\section{8}

Identification of Stem Cell Hierarchies in a Prostate Cancer Cell

LJ Flynn, O Sheils, C Spillane, C O'Brien, J O'Leary, S Finn. Trinity College Dublin, Dublin, Ireland; St. James' Hospital, Dublin, Ireland.

Background: Emerging evidence supports the concept that a biologically distinct, rare population of cells, termed cancer stem cells are integral to the initiation and perpetuation of several forms of human cancer. In order to elucidate the potential mechanisms responsible for the intrinsic properties of prostate cancer stem cells, such as high proliferative capacity, we have optimised a clonogenic assay using single cell sorting to generate a spectrum of morphologically distinct colonies similar to those produced by stem cells in normal epithelia. When plated at low density, a prostate cancer cell line (PC3 ) gives rise to morphologically heterogeneous colonies of differing growth potentials; classified as holoclones, meroclones and paraclones. Holoclones assume a compact, highly organised shape while meroclones and paraclones form irregular colonies. We show that PC-3 holoclones highly express two canonical markers of stemness, Nanog and OCT4. Furthermore, we have shown that cells isolated from holoclones are capable of regenerating all colony patterns. We have also shown the feasibility of preparing a small RNA library for next generation sequencing from a single holoclone.

Design: The prostate cancer cell line PC-3 was plated at low density using a high speed single cell sorter. Following plating, a proportion of the cells died while the remainder developed small colonies within 5 days, which displayed varying morphologies within 1 week of plating. The colonies were classified based on their appearance and harvested no more than 14 days after cell sorting. The expression of Nanog and OCT4 was examined in the holoclones in order to verify their stem-like properties. Holoclones were also harvested and dissociated in order to flow sort and plate in the same manner. The RNA was analysed to determine input quantities for small RNA library construction. Results: When plated at low density, PC-3 prostate cancer cells generate a range of morphologically unique colonies of varying proliferative potential. These colonies parallel those generated by stem and amplifying cells of the normal epithelial compartment. When compared to the parental cell line, PC-3 holoclones highly express Nanog and OCT4; two critical stem cell markers. Furthermore PC-3 holoclones are capable of regenerating all colony types.

Conclusions: Cancer stem cell characteristics are enriched in holoclone morphology of the $\mathrm{PC}-3$ prostate cancer cell line, indicating that the propagation of holoclones by single cell cultivation may allow for the in vitro elucidation of cancer stem cell mechanisms. 


\section{Clonal Evaluation of Prostate Cancer Biopsies by Dual ERG/SPINK1}

\section{Immunohistochemistry}

J Fontugne, K Davis, MA Rubin, JM Mosquera, SA Tomlins. Weill Medical College of Cornell University, New York, NY; University of Michigan Medical School, Ann Arbor, MI

Background: Two or more foci of prostate cancer (PCa) separated by intervening benign tissue in a single core is a well-recognized finding on prostate biopsy (PB). To report this finding, the pathologist can measure discontinuous foci as if they were one continuous focus or only measure the area actually involved by tumor foci. This would have significant impact on patient enrollment in active surveillance (AS) protocols. Some authors propose including intervening benign tissue, suggesting it reflects tumor volume at radical prostatectomy. We hypothesize that spatially distinct $\mathrm{PCa}$ foci in biopsies may arise from separate clones, impacting assessment of tumor extent.

Design: 97 prostate biopsy cores from 80 cases with spatially distinct tumor foci (discontinuous involvement) were studied. To determine their clonal status, dual ERG/ SPINK1 IHC staining was performed.

Results: Discontinuous tumor involvement, including benign intervening tissue, ranged from 15 to $100 \%$. Gleason scores ranged from 6 to 9 . Out of the 97 core biopsies with discontinuous involvement, 24 harbored clonally distinct foci $(24.7 \%)$ as follows:, 1$)$ $58.3 \%(14 / 24)$ had one ERG+ focus, and one ERG-/SPINK1- focus, 2) $29.2 \%(7 / 24)$ had one SPINK1+ focus and one ERG-/SPINK1- focus and 3) $12.5 \%(3 / 24)$ had one ERG+ focus and one SPINK1+ focus. ERG and SPINK1 overexpression were mutually exclusive.

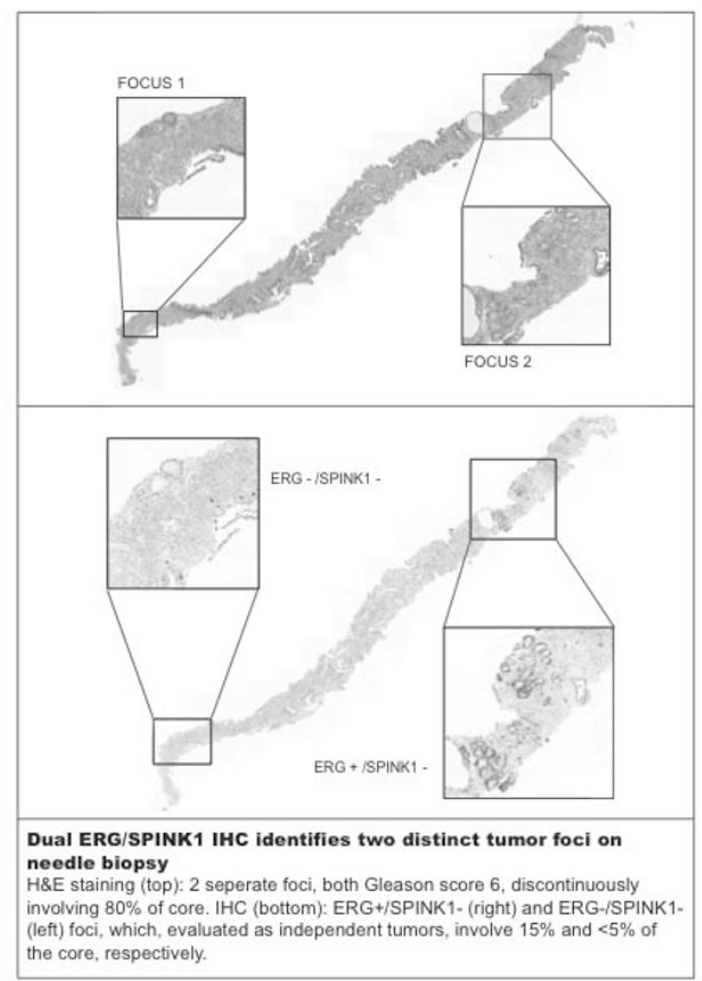

Conclusions: $22 \%$ of PB cores with discontinuous tumor involvement showed discordant ERG/SPINK1 status in separate foci, consistent with multiclonal disease. These results have significant impact in assessment of tumor involvement at diagnosis. We hypothesize that multiclonal discontinuously involved cores may be associated with smaller, multiclonal tumor foci and prostatectomy. Hence multiclonality assessment in discontinuously involved prostate biopsy cores through ERG/SPINK1 IHC may increase the number of eligible patients for AS. Validation and additional inter-institutional studies are ongoing.

940 Molecular Subclasses of Prostate Cancer: A Comprehensive Evaluation of All Tumor Foci in Radical Prostatectomy Specimens

$J$ Fontugne, $H$ Ye, K Park, M Blattner, J Siddiqui, T MacDonald, AM Chinnaiyan, M Sanda, CE Barbieri, MA Rubin, JM Mosquera. Weill Medical College of Cornell University, New York, NY; Weill Medical College of Cornell University and New York Presbyterian Hospital, New York, NY; Beth Israel Deaconess Medical Center, Boston, MA; University of Michigan Medical School, Ann Arbor, MI; Harvard Medical School, Boston, MA.

Background: Prostate cancer ( $\mathrm{PCa}$ ) is a multifocal, highly heterogeneous disease. Similar to other malignancies, identification of molecular subclasses for risk stratification and therapeutic management is needed. Reported genomic alterations such as $E R G$ rearrangement, $P T E N$ deletion, $C H D 1$ loss and SPOP mutations, as well protein expression alterations, such as SPINK1 overexpression, are recurrent in prostate $\mathrm{PCa}$. The goal of this study was to evaluate these aberrations in each tumor nodule of radical prostatectomy (RP) specimens, to better understand molecular subclasses of $\mathrm{PCa}$ in the context of multifocality.
Design: 258 RP specimens collected prospectively from the well-characterized Early Detection Research Network trial were included. All tumor foci identified on H\&E were interrogated using dual ERG/SPINK1 IHC, and FISH to determine $E R G$ rearrangement, PTEN and CHDI deletion status. SPOP mutation status was screened by High Resolution Melting curve and confirmed by Sanger Sequencing on a subset of cases. Results: These 258 RP cases represented 624 tumor foci. 185/258 cases ( $72 \%$ ) were multifocal: the number of foci ranged from 2 to 10 and Gleason scores ranged from 6 to $10.53 \%(98 / 185)$ of multifocal cases were molecularly heterogeneous. $56 \%$ and $18 \%$ of cases showed $E R G$ rearrangement and SPINK1 overexpression, respectively, in at least one tumor focus. PTEN loss was present in $12 \%$ and an SPOP mutation was present in $10 \%$ of cases (missense mutations at F133L, F133V, F102V, F102G). CHDI deletion was seen in $7.3 \%$ of cases. ERG rearrangement and SPOP mutation were mutually exclusive on a tumor focus basis, but concurrently present within one RP. ERG fusion and SPINK1 overexpression were mutually exclusive.

Conclusions: These results illustrate the concept of multiclonality of PCa. Molecular characterization of each nodule furthers our understanding of heterogeneity and supports the molecular classification of PCa. Further studies to validate clinical correlation of these assays are ongoing.

941 Recurrent Prostate Cancer Genomic Alterations Predict Response to Brachytherapy Treatment

J Fontugne, D Lee, C Cantaloni, T MacDonald, CE Barbieri, O Caffo, E Hanspeter, G Mazzoleni, P Dalla Palma, MA Rubin, G Fellin, JM Mosquera, M Barbareschi, F Demichelis. Weill Medical College of Cornell University, New York, NY; University of Trento, Trento, Italy; Ospedale Santa Chiara, Trento, Italy; Central Hospital, Bolzano, Italy.

Background: The discovery of $E R G$ gene rearrangements and recurrent associated molecular alterations, such as PTEN deletions, has helped improve the understanding of prostate carcinogenesis. However, molecular stratification in prostate cancer has not been well studied in response to different treatment modalities. In this study, we sought to evaluate the association between ERG gene rearrangements and PTEN deletions with oncologic outcomes in patients with prostate cancer treated with brachytherapy. Design: Ninety-two men underwent brachytherapy with I-125 at a delivered dose of 145 Gy between 2000 and 2008 at three centers in Northern Italy. Pre-treatment prostate biopsy samples were obtained and analyzed by IHC and FISH for the presence of $E R G$ rearrangement and overexpression, and PTEN deletion and loss of expression. Univariable and multivariable Cox-regression analyses evaluated the association of $E R G$ and PTEN status with biochemical recurrence (BCR).

Results: Within a median follow-up of 73 months, 10 patients (11\%) experienced BCR. Of the 80 samples that had IHC and FISH performed for $E R G, 46(57.8 \%)$ demonstrated rearrangement by FISH and $45(56.3 \%)$ overexpression by IHC. Of the 77 samples that had IHC and FISH for PTEN, $14(18.2 \%)$ had PTEN deletion by FISH and 22 (28.6\%) by IHC. There were no significant associations between $E R G$ or PTEN status and clinicopathologic features. Patients with concurrent $E R G$ rearrangement and PTEN deletion status demonstrated significantly worse relapse free survival rates compared to those who were $E R G$ wild-type or PTEN wild-type ( $<0.01$ ). In multivariable Cox regression analysis adjusted for the effects of standard clinicopathologic features, the combination of $E R G$ rearranged and PTEN deletion was independently associated with BCR (HR 2.6, $\mathrm{p}=0.02$ )

Conclusions: The concurrent presence of ETS gene rearrangements and loss of PTEN was independently associated with time to BCR and worse prognosis in patients under going brachytherapy. Identifying patients in this molecular subclass may improve treatment personalization and determine those who require more aggressive therapy. Future validation studies are needed to validate the molecular subtyping of prostate cancer for risk-stratification.

\section{Monoclonal Antibody F77 Is Prevalent in Non-Prostatic Tumors}

R Frank, MI Greene, P Lal. Hospital of the University of Pennsylvania, Philadelphia, PA. Background: Monoclonal antibody F77 (mAb F77) has been shown to recognize a unique glycolipid antigen highly restricted to prostate cancer cell surface, called prostate cancer lipid antigen (PCLA). The unique binding pattern of mAb F77 indicated that PCLA exists predominantly in prostate and that its expression is consistently higher in tumor tissues than in normal tissues. However the expression of PCLA as identified by mAb F77 has not been fully explored in other tumors.

Design: This is a single center retrospective study of 418 primary and metastatic carcinomas originating from the ovary, lung, bladder, prostate, and kidney. Tissue microarrays composed of $5 \mathrm{~mm}$ cores from representative tumor formalin-fixed paraffin embedded sections were made. Immunohistochemical analysis of antibodies against F77 (1:200 dilution) was performed on the tissue microarrays using standard immunohistochemical protocols. mAb F77 was considered positive with either membranous or cytoplasmic staining. mAb F77 staining intensity in tumor cells was recorded semiquantitatively as $1+$ through $3+$, with only $2+$ and $3+$ staining intensity considered positive. The percentage of positive tumor cells was also noted.

Results: $\mathrm{mAb} F 77$ tissue microarrays of primary and metastatic carcinomas originating from the ovary $(\mathrm{N}=108)$, lung $(\mathrm{N}=34)$, bladder $(\mathrm{N}=57)$, prostate $(\mathrm{N}=134)$, and kidney $(\mathrm{N}=80)$ were analyzed for staining intensity of tumor cells. All renal tumors were negative $(0 / 80)$. Lung $(31 / 34,91 \%)$, bladder $(33 / 57,58 \%)$, prostate $(134 / 139$, $96 \%$ ), and ovary $(69 / 108,64 \%)$ had higher rates of mAb F77 staining. Interestingly, ovarian primary carcinomas has a higher frequency of mAb F77 positivity (34/48, $71 \%$ ) than ovarian carcinoma metastases $(35 / 60,58 \%)$. Also of note, the staining pattern was different between lung (predominantly membranous staining, 6/31) and bladder carcinomas (predominantly nuclear and cytoplasmic staining, 33/33), while ovarian carcinoma had mixture of cytoplasmic and membranous staining, likely due to the variety of tumor types. 
Conclusions: Our finding suggests that mAb F77 cannot be used as a unique marker of prostatic adenocarcinoma. mAb F77 can be seen in a variety of carcinomas. While the biologic significance of mAb F77 targeting proteins in these neoplasms remains unclear, the findings could help aid in further defining proteins expressed on a number of tumors. Additionally, the staining pattern of mAb F77 may guide insight into the pathophysiology of carcinomatosis and metastasis.

943 CHD1 Deletion in a Cohort of Castration-Resistant Prostate Cancer CS Friedman, F-M Deng, CE Barbieri, T MacDonald, WXu, J Melamed, MA Rubin, JM Mosquera, MZhou. NYP-Weill Cornell Medical College, New York, NY; New York University Langone Medical Center, New York, NY; Institute for Precision Medicine, Weill Cornell Medical College/NYPH, New York, NY.

Background: Recent sequencing studies have demonstrated CHD1 alterations in castration-resistant prostate cancer (CRPC). Mutations and deletions of CHD1, which encodes a chromatin-modifying enzyme, appear to define a molecular subtype of prostate cancer (PCa): CHD1 deletion occurs at a higher rate in ETS fusion-negative tumors and is frequently altered in SPOP mutant PCa. In this study we sought to investigate the deletion status of CHD1, as well as ERG and PTEN status, in a well-characterized cohort of CRCP.

Design: The study group consisted of 35 CRPC tumor samples from 18 patients with advanced high volume disease who underwent transurethral resection, 6 patients who underwent biopsy of metastatic sites, and 11 CRPC patients with clinically localized $\mathrm{PCa}$ who received treatment followed by salvage prostatectomy. The treatment in all cases was surgical castration, androgen deprivation therapy, or both. Controls consisted of tumor samples from 35 patients with hormone-naive localized or metastatic PCa. TMAs were constructed and cases were interrogated for CHD1 deletion (FISH), ERG translocation (FISH and IHC), and PTEN loss (CISH and IHC). All the methods have been previously described.

Results: CHD1 deletion was present in 11/35 CRPC (31\%), two of which had homozygous deletion. In contrast, only $4 / 35$ hormone-naive cases harbored CHD1 deletion $(11 \%)$, all of them hemizygous $(\mathrm{P}<0.05)$. ERG rearrangement was present in $19 / 35$ CRPC $(54 \%)$, and in 9/35 hormone naive tumors $(26 \%)(\mathrm{P}<0.05)$. CHD1 deletion and ERG rearrangement were mutually exclusive $(\mathrm{P}<0.05)$. There was full concordance between ERG FISH and IHC results. PTEN deletion and loss of expression was present in $8 / 35$ CRPC cases (22\%); 6/35 hormone-naive cases (17\%) had PTEN loss.

Conclusions: CHD1 is deleted at a significantly higher rate in CRPC compared to hormone naive PCa; our cohort shows higher rates of CHD1 deletion in CRPC than previous studies. CHD1 deletion occurs in ERG-negative tumors. It is known that CHD1 alterations cause impairment of androgen receptor (AR)-dependent transcription and are more frequent in CRPC. The low frequency of PTEN loss in CHD1 deleted CRPC cases in our cohort is confounding; it is likely related to few metastatic samples being interrogated. Further molecular studies are ongoing.

\section{Ddx5 Expression Is Linked with ERG-Positive Prostate Cancer but Lacks Substantial Impact on Clinicopathological Parameters of Patients Treated with Prostatectomy}

B Furusato, H Takahasi, S Sato, M Okayasu, M Owada, T Kimura, SEgawa, M Ikegami. Jikei University School of Medicine, Tokyo, Japan.

Background: The DEAD box RNA helicase p68 (Ddx5) has been implicated in various cellular processes and shown to possess transcriptional co-activator function. Clark et al. (Cancer Res. 2008) reported that Ddx 5 is an important androgen receptor (AR) transcriptional co-activator in prostate cancer $(\mathrm{PCa})$ and is over-expressed in the prostate in later stages of the disease. Given the high prognostic potential of Ddx 5 in PCa based on earlier literature, we utilized Asian PCa specimens to validate these earlier findings and compared Ddx5 with ERG expression status and clinicopathological features.

Design: Ddx 5 expression was analyzed by immunohistochemistry on a tissue microarray containing $128 \mathrm{PCa}$ specimens. The results were compared with clinicopathological parameters, including stage, grade and ERG status.

Results: Ddx5 expression was elevated in PCa and reduced in benign prostatic glands. Ddx 5 staining was found in $38.7 \%$ (46/106) of interpretable cases. Among the Ddx5positive cases, staining was considered strong, moderate, or weak in $4.3 \%(2 / 46)$, $32.6 \%(15 / 46)$ or $63 \%(29 / 46)$ of PCa cases, respectively. When Ddx 5 expression was categorized as positive or negative, positive staining was significantly associated with ERG expression $(p<0.0001)$. However, Ddx5 expression was marginally significant with tumor grade (Gleason score) and stage $(p<0.046)$.

Conclusions: Ddx 5 expression in ERG-positive prostate cancers may provide further evidence for marked biological differences between "fusion type" and "non-fusion type" prostate cancer. However, there was weak association between Ddx 5 expression and clinicopathological parameters in our cohort of Asian samples. Further studies of larger cohorts are required to understand the molecular link between cases with concomitant Ddx5 and ERG expression.

\section{Comprehensive Evaluation of the Urothelial Carcinoma Markers GATA3 and S100P in Squamous Cell Carcinoma}

MP Gailey, AM Bellizzi. University of Iowa, Iowa City, IA.

Background: Urothelial carcinoma (UC) and squamous cell carcinoma (SCC) demonstrate substantial morphologic and immunophenotypic overlap. SCCs usually express p63 and CK5/6, while UCs usually and often do, respectively. Co-expression of CK7 and CK20, though specific for UC, is only moderately sensitive. "Second generation" urothelial markers thrombomodulin and uroplakin III failed to attain widespread use due to modest sensitivity. More recently, GATA3 and S100P have been touted as extremely sensitive/specific for the diagnosis of UC, though extensive testing of SCCs has not been undertaken. The purpose of this study is to document expression of GATA3 and S100P in a large cohort of SCCs from diverse anatomic sites.

Design: GATA3 and S100P immunohistochemistry was performed on whole sections from 262 SCCs and 50 UCs. Any definite GATA3 nuclear staining and granular cytoplasmic and/or nuclear S100P staining in $\geq 5 \%$ of tumor cells were considered positive. Extent $(\%)$ and intensity $(0,1+, 2+, 3+)$ were also evaluated and an $\mathrm{H}$-score (extent $\mathrm{x}$ intensity) calculated. Sensitivity and specificity were calculated. Fisher's exact test and the Mann-Whitney test were used as appropriate, with $\mathrm{p}<0.05$ considered significant.

Results: GATA3 expression was seen in $21 \%$ of SCCs and $84 \%$ of UCs $\left(\mathrm{p}<1 \times 10^{-4}\right)$. While expression in UCs tended to be diffuse/strong (mean H-score in positives 228), in SCCs it was typically weaker/patchier (mean H-score in positives 80$)\left(\mathrm{p}<1 \times 10^{-4}\right)$. S100P-positivity was seen in $4 \%$ of SCCs and $60 \%$ of UCs $\left(\mathrm{p}<1 \times 10^{-4}\right)$, which again tended to be stronger in the latter (mean $\mathrm{H}$-scores in positives 32 and 127, respectively) $\left(\mathrm{p}=1 \times 10^{-3}\right)$. Expression of GATA3 was enriched in genitourinary SCCs $(31 \%)$. Detailed expression data by site are presented in the Table.

\begin{tabular}{|c|c|c|}
\hline & GATA3 (n, \%) & S100P (n,\%) \\
\hline Anus & $7 / 19(37 \%)$ & $0 / 19(0 \%)$ \\
\hline Bladder (UC) & $42 / 50(84 \%)$ & $30 / 50(60 \%)$ \\
\hline Cervix & $7 / 32(22 \%)$ & $4 / 32(13 \%)$ \\
\hline Esophagus & $3 / 24(13 \%)$ & $1 / 24(4 \%)$ \\
\hline Larynx & $0 / 27(0 \%)$ & $0 / 27(0 \%)$ \\
\hline Lung & $0 / 31(0 \%)$ & $0 / 31(0 \%)$ \\
\hline Penis & $8 / 28(29 \%)$ & $4 / 28(14 \%)$ \\
\hline Skin & $10 / 23(43 \%)$ & $4 / 23(17 \%)$ \\
\hline Ventral Tongue/Floor of Mouth & $0 / 26(0 \%)$ & $1 / 26(4 \%)$ \\
\hline Vagina & $13 / 26(50 \%)$ & $0 / 26(0 \%)$ \\
\hline Vulva & $6 / 26(23 \%)$ & $1 / 26(4 \%)$ \\
\hline
\end{tabular}

Overall, GATA3 was $84 \%$ sensitive, $79 \%$ specific and S100P $60 \%$ sensitive, $96 \%$ specific for the diagnosis of UC (vs SCC).

Conclusions: While GATA 3 is more sensitive for the diagnosis of UC, S100P is more specific, with frequent GATA3 expression in genitourinary SCCs. Attention to extent and intensity of staining may help "rescue" the specificity of GATA3, though pathologists are cautioned against using either marker alone when considering a differential including SCC and UC.

\section{Cytogenetic and Histopathologic Correlation of Renal Epithelial} Neoplasms

$J$-A Gardner, $M$ Tang, $K$ Cooper. Fletcher Allen Health Care/UVM, Burlington, VT; University of Pennsylvania, Philadelphia, PA.

Background: Recurrent chromosomal abnormalities have been well documented in renal cell carcinoma (RCC). Specific cytogenetic changes correlate with the histologic subtypes of RCC, and thus can serve as a useful diagnostic tool for the surgical pathologist, and may prevent potential misclassification of tumors. Our study evaluates the growth rate and cytogenetic changes found in a series of 421 renal tumors, and identifies cases with histopathologic-cytogenetic discrepancies.

Design: Fresh tissue from 421 renal tumors was submitted for chromosome analysis over a 9.5 year period at our institution. Cytogenetics was successful in $371(88 \%)$. Cytogenetic and surgical pathology reports were compared for each case. Cases with discrepant cytogenetic and histopathological results were identified and reviewed. In 16 cases $(8 \%)$ the preliminary surgical diagnosis was changed based on the cytogenetic findings. Eight Samples (50\%) were identified histologically as conventional RCC; however, cytogenetic findings were consistent with papillary RCC. Cases were grouped into the following categories: clear cell, papillary, oncocytoma, chromophobe, urothelial, collision, and other

Results: Normal karyotypes were found in $176(47 \%)$ of the 371 samples. Abnormal karyotypes were found in $195(53 \%)$. In $169(86 \%)$ of the abnormal cases, the cytogenetic changes were classic for a given tumor type: clear cell (89\%); papillary (79\%); oncocytoma (50\%); chromophobe, urothelial, collision and other $(100 \%)$. The most common non-classic abnormalities were nonspecific gains and losses of chromosomes and complex structural abnormalities.

Conclusions: Cytogenetic analysis is a useful ancillary tool in the diagnosis of RCC. Our series shows subtyping RCC on histology alone can be challenging, with histologiccytogenetic correlation identifying an $8 \%$ misdiagnosis rate. In addition to improving diagnostic accuracy for RCC, cytogenetic-histologic correlation has allowed us to recognize the most problematic areas in diagnosing RCC. In our institution, the most challenging distinction is between conventional RCC and papillary RCC with a solid architecture and/or clear cell cytomorphology. We will ultimately use this information as a quality assurance measure to provide more accurate diagnoses for RCC, and as a quality improvement effort to further educate our surgical pathologists on the histologic subtleties of these neoplasms.

\section{Pathologic Findings in Patients with Targeted MRI Guided Prostate} Needle Core Biopsies

RL Geller, SG Nour, AO Osunkoya. Emory University School of Medicine, Atlanta, GA. Background: In contrast to the systematic (non-targeted) sampling approach of transrectal ultrasound-guided biopsies (TRUS-GB), targeted magnetic resonance imaging-guided biopsies (TMRI-GB) target regions of the prostate suspicious for prostate cancer (PCa), based on findings on multiparametric MRI. There are limited studies in the literature examining the pathologic findings identified following TMRIGB

Design: A search was made through our Urologic Pathology files for prostate needle core biopsies that were obtained via TMRI-GB from 2012-2013. 
Results: Fourteen cases were identified. Mean patient (PT) age was 61 yrs (range: 51 $73 \mathrm{yrs})$. Nine of 14 (64\%) PTs had a history of PCa, 2/14 (14\%) PTs had a history of negative TRUS-GB and rising PSA levels, and 3/14 (22\%) PTs had never undergone biopsy. Of the 9 PTs with previous PCa diagnosis, 6 had a Gleason score of $3+3=6$ (on active surveillance), 2 had a Gleason score of $7(3+4=7$ and $4+3=7)$, and 1 had ductal $\mathrm{PCa}$, Gleason score $4+4=8$ (these last three PTs were considering focal therapy). All PTs were described as having at least one "focal abnormality" or "foci suspicious for PCa" on preliminary diagnostic MRI. A total of 45 lesions were sampled, and 140 cores were evaluated by TMRI-GB. The mean number of cores sampled per PT with TMRI-GB was 10 (range: $3-18$ cores), and the average number of cores per targeted lesion sampled was 3 cores (range: $1-5$ cores). TMRI-GB results were positive for PCa in 8/14 (57\%) PTs (only previously "benign" areas were sampled in these PTs that already had a diagnosis of PCa). Of the 8 PTs with positive TMRI-GB, 5 had previous diagnoses of PCa and 3 had no prior biopsy. The Gleason scores in these 8 PTs were as follows; 3 had Gleason score $3+3=6,2$ had a Gleason score of $7(3+4=7$ and the other had mixed ductal PCa and conventional PCa, Gleason Score $4+3=7$ ), 1 had a Gleason score of $4+4=8$ and 2 had a Gleason Score $4+5=9$. PCa involvement of $50 \%$ of the core was identified in $6 / 8$ PTs. Of the 6 PTs with benign TMRI-GB, 4 had a previous diagnosis of cancer (note that it is the previously benign areas in these PTs that were sampled), and 2 had previously benign TRUS-GB and prostate specific antigen $>4 \mathrm{ng} / \mathrm{ml}$.

Conclusions: TMRI-GB may have a critical role in PTs on active surveillance in which due to detection of previously unsampled foci with higher Gleason scores, higher percentage of tumor involvement and higher number of cores involved, may have to be taken off this protocol. TMRI-GB also has role in tumor mapping for PTs considering focal therapy (e.g. laser ablation), and primary detection of $\mathrm{PCa}$

\section{Does BAP1 Play an Important Role in Hereditary Kidney Cancers?} A Ghosh, K Lara-Otero, MJ Merino. National Institutes of Health, Bethesda, MD. Background: Hereditary renal cell carcinomas (RCC) constitute about $5 \%$ of all RCCs. These include, VHL, HLRCC, BHD, Oncocytoma, Papillary Type 1, TSC, SDHB and RCCs and others. Several genes, including $V H L, M E T, F L C N, F H$ and genes encoding the succinate dehydrogenase ( $\mathrm{SDH}$ ) subunits $\mathrm{B} / \mathrm{C} / \mathrm{D}$ have been identified as causative. However, the genetic basis of a significant percentage of familial RCC, specially clear cell type, remain unknown. BAPl (BRCA1 associated protein-1), a tumor suppressor gene that encodes a nuclear deubiquitinase, is inactivated in $15 \%$ of clear cell RCCs and its loss was associated with high tumor grade. We investigated the possible role of this gene in the spectrum of RCC part of the Hereditary syndromes.

Design: To elucidate the role of BAP1 in all the different varieties of hereditary RCC, we studied a panel of RCCs which included 8 VHL tumors, 8 HLRCCs, 5 chromophobe, 8 papillary Type 1,5 Oncocytomas, 3 SDHB, 3 BHD, and 20 sporadic clear cell RCCs. To analyze the BAP1 expression in these tumors, formalin fixed paraffin embedded (FFPE) tissues were immunostained with mouse monoclonal anti-human BAP1 antibody (Clone C-4, Santa Cruz). Microdissected tumor DNA was used for PCR amplification and sequencing of $B A P 1$

Results: We found that all the tumors except two showed positive nuclear staining for BAP1. The two negative cases that were negative for BAP1 were Clear cell type and belonged to two siblings. Molecular analysis showed both patients harboring the p.L14H mutation.

Conclusions: Our study supports the hypothesis that $B A P I$ mutations can play a role in hereditary syndromes predominantly in clear cell tumors. Staining for BAP1 should be done when there is no definite know mutation in a clear cell cancer. The two related patients who had mutations had aggressive, metastatic disease, which suggests that probably $B A P I$ does play a role in certain aggressive hereditary RCC types.

949 Standard 12 Core Biopsies Undersample Grade and Volume of at Least a Third of Anterior Predominant Prostate Cancers: Implications for Active Surveillance Trials

SK Gill, J Wright, W Ellis, LD True. University of Washington, Seattle, WA.

Background: The decision to recommend intent-to-cure therapy for men with primary prostate cancer (PC) is based, in part, on the volume and grade of cancer in needle core biopsies (NCB). Patients with Gleason score 6 cancers that are present in a limited number of core biopsies are candidates for active surveillance. However, a subset of these men will have large volume cancer that is undersampled by the standard NCB approach since the cancers are centered in the less accessible anterior region of the prostate. In this study we determine the fraction of anterior PC's whose volume and grade are undersampled by the current standard 12 NCB approach.

Design: We searched our pathology database between 2001 and 2013 for prostatectomy (RP) cases for which paired biopsy data was available. Tumors that were predominantly anterior in location (defined as $>75 \%$ of the cancer was anterior) were reviewed. Volume and grade of cancer in NCBs (volume was defined as the number of cancer-positive biopsies) and in RPs were correlated in anterior-predominant prostate cancers. Results: Of 1,117 radical prostatectomy specimens in our database, the cancer was located in the anterior region of the prostate in 94 cases. The median weight of the prostate in these cases was $42 \mathrm{gm}$ (range $16-175 \mathrm{gm}$ ). Median tumor volume was $2.9 \mathrm{cc}$ (range $0.1-11 \mathrm{cc}$ ). Of the 94 anterior-predominant cases, cancer involved the following number of cores per case: one core in $31 \%$, two in $29 \%$, three in $3 \%$, and four or more cores in $37 \%$ of cases. Limiting our analysis to the 61 anterior-predominant cancers for which the pathology of the NCBs met criteria for many active surveillance trials (Gleason score 6 cancer in no more than $3 \mathrm{NCBs}$ ), the volume of cancer in the RP exceeded 0.5 cc (a volume that is regarded by some clinicians as "clinically insignificant") in $96 \%$ of cases and the grade of cancer was Gleason score 7 in 38\% of cases.
Conclusions: A standard 12 needle core biopsy approach often underestimates the volume and grade of prostate cancers that are located in the anterior prostate. In our study anterior cancer volume was greater than $0.5 \mathrm{cc}$ in most cases and grade was higher than Gleason score 6 in at least a third of cases. In retrospect, these patients might not be candidates for active surveillance. A challenge confronting all of us involved in active surveillance trials is to more accurately sample anterior cancers for their grade and volume.

950 Urinary Bladder Paragangliomas: How IHC Can Assist to Identify Patients with SDHB Mutations

A Giubellino, K Lara-Otero, V Martucci, T Huynh, P Agarwal, K Pacak, MJ Merino. National Cancer Institute, Bethesda, MD; NICHD, Bethesda, MD; NCI, Bethesda, MD. Background: Urinary bladder paraganglioma (PGL) is an unusual tumor that originates from chromaffin cells of the sympathetic system of the urinary bladder wall. It accounts for less than $0.05 \%$ of all bladder neoplasms, often occurs in young adults with a female prevalence, and can be part of hereditary syndromes such as VHL, MEN and SDH. Germline mutations in the succinate dehydrogenase subunit B gene (SDHB) have been associated with hereditary forms of bladder PGL. Since SDHB mutations are associated with the highest rate of malignancy (above $50 \%$ ), accurate tumor characterization for SDHB gene mutation status is of utmost importance for the management and prognosis of these patients. We investigated the usefulness of SDHB protein staining by immunohistochemistry (IHC) as a diagnostic tool to identify bladder PGLs associated with SDHB gene mutations.

Design: Fourteen cases of urinary bladder PGLs were studied. All patients presented with clinical history suggestive of the presence of bladder PGL. Females predominated, with an average age of 28 . Tumors were morphologically evaluated and 10 were stained for SDHB protein expression by IHC. Two different rabbit polyclonal antibodies (HPA002867 and HPA002868, Sigma-Aldrich) were used to recognize different binding sites of the target human protein.

Results: Six out of ten tumors were negative for SDHB staining by IHC showing no cytoplasmic staining in the tumor cells when compared with the normal tissues. Five out these six cases had negative staining and were confirmed positive for germline SDHB mutations. Four of the 10 stained cases showed strong cytoplasmic staining and were negative for the presence of SDHB mutation. These patients were apparently sporadic or part of VHL syndrome. Only one case showed a negative staining and no germline SDHB mutation, however, further investigation of the tumor using complete genotyping of the SDHB gene using multiplex ligation-dependent probe amplification (MLPA) revealed a somatic SDHB gene deletion.

Conclusions: Our results indicate a direct correlation between the presence of an SDHB mutation, whether germline or sporadic, and negative SDHB IHC staining in urinary bladder PGLs. These findings strongly support the use of SDHB IHC to recognize PGL of urinary bladder associated with SDHB syndrome that may have potential for an aggressive behavior. Identification of these patients will also allow for a family screening and establishment of proper therapies.

951 Cystic Trophoblastic Tumor of the Testis: A Spontaneous Testicular and Postchemotherapy Neoplasm

$D$ Gondim, L Cheng, C Jones, T Ulbright, $M$ Idrees. Indiana University School of Medicine, Indianapolis, IN.

Background: Cystic trophoblastic tumor (CTT) has been described in postchemotherapy retroperitoneal lymph node dissections (PCRPLND) of patients with testicular germ cell tumors (GCT). Prognostically, this lesion is similar to teratoma and no further treatment is required after surgery in the absence of other components. CTT has not, however, been reported in the testis without prior treatment. We reviewed our experience with CTT, concentrating on testicular cases.

Design: An electronic data search from institutional files was performed for CTTs. Diagnosis was confirmed by reviewing H\&E slides; immunostains for inhibin, hCG, and p63 were performed on available blocks or unstained slides. Clinical and followup data were obtained from clinical notes.

Results: We identified 11 testicular CTTs ( 8 consultation and 3 in-house) from 1996-2013. Additionally, 26 and 8 cases of CTTs from 2000-2013 were identified from PCRPLND and other metastatic sites, respectively. The patients with testicular CTT were $15-43$ years old (median, 24$)$. The CTT was a minor component $(<1 \%-10 \%)$ and associated with other GCTs: teratoma (11), embryonal carcinoma (4), yolk sac tumor (4), and choriocarcinoma (1). CTT and teratoma were also found in 2 subsequent resections (spermatic cord and pelvis) from these patients. Five patients had preorchiectomy chemotherapy because of metastatic disease. Four patients had no prior therapy. No information was available for 2 cases. The CTTs were cystic to partly solid with small to large cysts often containing fibrinoid material and lined by mononucleated squamoid cells with eosinophilic to pale, frequently vacuolated cytoplasm. The nuclei were pleomorphic with dense, often smudged chromatin; mitotic activity was inconspicuous. Immunostains on 5 cases demonstrated focally positive hCG and inhibin and uniformly negative p63. All 26 CTTs in RPLNDs (range, 17-40 yrs; median, 26 yrs) had pre-resection chemotherapy and were associated with teratoma. Yolk sac and rhabdomyomatous tumors were present in 1 case each. Moreover, in 8 cases, CTTs were found in lung (3), liver (2), pelvis (1), cervical lymph node, (1) and mediastinum (1) in association with teratoma (4) and/or necrosis (4). The original orchiectomy had choriocarcinoma in only 4 cases of metastatic CTT.

Conclusions: The pathogenesis of CTT is not clear. Because untreated patients without choriocarcinoma may have CTT in the testis, it may not be a form of regressed choriocarcinoma. Its squamoid morphology may cause it to be misinterpreted as squamous cell carcinoma, as occurred in 1 of our testicular cases. 
952 Low ARID1A Expression in Superficial Non-Muscle Invasive Urothelial Carcinoma of Urinary Bladder

$N$ Gonzalez-Roibon, SF Faraj, E Munari, SM Bezerra, I-M Shih, TJ Bivalacqua, MP Schoenberg, GJ Netto, K Rezaei. Johns Hopkins Medical Institutions, Baltimore, MD; George Washington University, Washington, DC

Background: AT-rich interactive domain 1A(ARID1A) is a tumor suppressor gene that has been described to be inactivated in several type of neoplasms, including epithelial ovarian and endometrial carcinomas. ARID1A loss has been correlated with prognosis. Recently, using a cystectomy cohort, we demonstrated low ARID1A expression to be associated with lower stage tumors and a favorable prognosis. The current study assesses ARID1A expression in transurethral resection of bladder (TURB) specimens for non-muscle invasive bladder cancer (NMI-BC)

Design: Three tissue microarrays were constructed from 54 NMI-BC from one of the authors' institution. Nuclear ARID1 A staining (HPA005456, Sigma-Aldrich, St Louis, $\mathrm{MO})$ was evaluated using immunohistochemistry. An $\mathrm{H}$-score was calculated as the sum of the products of intensity (0-3) multiplied by extent of expression (0 to $100 \%$ ). Average $\mathrm{H}$-score per case was used for statistical analysis. Low ARID1A expression was defined as $\mathrm{H}$-score below the third quartile.

Results: Median H-score was 228 (range: 60-298). We found low ARID1A expression in $75 \%$ (39 of 52) of NMI-BC. There was no difference between ARID1A expression in invasive and noninvasive carcinomas $(\mathrm{p}=0.65)$. There was no correlation between ARID1A expression and clinicopathologic features such as age at diagnosis $(\mathrm{p}=0.5)$, gender $(p=1)$, $p$ T category $(p=0.12)$, treatment with $B C G(p=0.73)$ or mitomycin $(\mathrm{p}=0.17)$. Rates of overall survival (OS), tumor recurrence-free survival and tumor progression-free survival (PFS) were $87.5 \%, 14.8 \%$ and $83.3 \%$, respectively. There was no association between ARID1A expression and OS (OR: 1.5, $\mathrm{p}=0.67)$, tumor recurrence-free survival (OR: $1, \mathrm{p}=1$ ) or PFS (OR: $0.83, \mathrm{p}=0.83$ ).

Conclusions: Low ARID1A expression is found in a high proportion of NMIBC, ARID1A expression levels in TURB specimens were not correlated with clinicopathologic parameters or outcome in our cohort of superficial bladder cancer.

953 Ureteral and Urethral Frozen Sections during Radical Cystectomy: The Significance of Denudation and Atypia

J Gordetsky, JI Epstein. Johns Hopkins Hospital, Baltimore, MD.

Background: While prior studies have looked at the utility of frozen section (FS) on ureteral and urethral margins in radical cystectomies, none have evaluated the meaning of a FS diagnosis of denudation or atypia (i.e. CIS cannot be ruled out).

Design: We searched our Surgical Pathology database from 2000-2012 for radical cystectomies. We compared FS diagnoses with the corresponding permanent section control as well as additionally submitted margins.

Results: We analyzed 1222 ureteral and 366 urethral FS in 822 radical cystectomy specimens. On FS for ureters, there were 56 diagnoses of atypia, 19 (33.9\%) of which showed CIS on permanent section, and 81 diagnoses of denudation, only $1(1.2 \%)$ of which showed CIS on permanent section. On frozen sections for urethras, there were 12 diagnoses of atypia, $2(16.7 \%)$ of which showed CIS on permanent section, and 17 diagnoses of denudation, only $1(5.9 \%)$ of which showed CIS on permanent section. Additionally, we evaluated if the finding of denudation or atypia on FS in either the ureter or urethra corresponded to a diagnosis of CIS either on permanent section or on an additionally submitted margin in a case. 23/60 (38.3\%) cases with atypia on FS had CIS either on permanent sections or on additionally submitted specimens. 14/93 $(15.0 \%)$ cases with denudation on FS had CIS either on permanent sections or on additionally submitted specimens. Compared to a negative FS diagnosis, a diagnosis of either atypia or denudation was predictive of the finding of CIS on either permanent sections or subsequent additional margins $(\mathrm{p}<.0001) .75 / 159(47.2 \%)$ of ureteral or urethral margins initially positive on FS were able to be converted to a final negative margin by the urologist resecting additional tissue.

Conclusions: For frozen sections of ureteral and urethral margins, a diagnosis of atypia corresponds to a high frequency of CIS on permanent section and is predictive of CIS on subsequent sections. Although a diagnosis of denudation on a frozen margin rarely converts to a diagnosis of CIS on permanent section, it is predictive of the finding of CIS on subsequently submitted additional sections. In addition, nearly half of all initially positive margins on frozen section are able to be converted to a final negative margin by additional resection of tissue. In summary, a FS diagnosis of atypia or denuded at time of cystectomy should prompt urologists to obtain additional margins which in about $1 / 2$ of cases can result in a final negative margin.

954 Pseudopapillary Features in Prostatic Adenocarcinoma Mimicking Urothelial Carcinoma: A Diagnostic Pitfall

J Gordetsky, JI Epstein. Johns Hopkins Hospital, Baltimore, MD.

Background: High grade prostatic adenocarcinoma (PCa.) can have overlapping morphologic features with high grade urothelial carcinoma (UC). Pseudopapillary features have not previously been described in high grade acinar $\mathrm{PCa}$.

Design: We reviewed our consults (2006-2013) for cases of high grade PCa., where Gleason score was $5+4=9$ or $5+5=10$, and the differential diagnosis included high grade UC.

Results: We identified 5 consult cases of high grade PCa. with pseudopapillary features, mimicking UC. Two cases were originally misdiagnosed as UC. In 2 cases, the outside diagnosis was UC versus PCa., and 1 case had no submitting diagnosis. All cases were TURs with tumor involving the prostatic urethra, 4 with bladder involvement. 2 patients had a known history of PCa. The tumors grew in nests and sheets with 1 having microacinar differentiation and another focal, rare glands. In places, tumors formed papillary-appearing structures with central blood vessels.

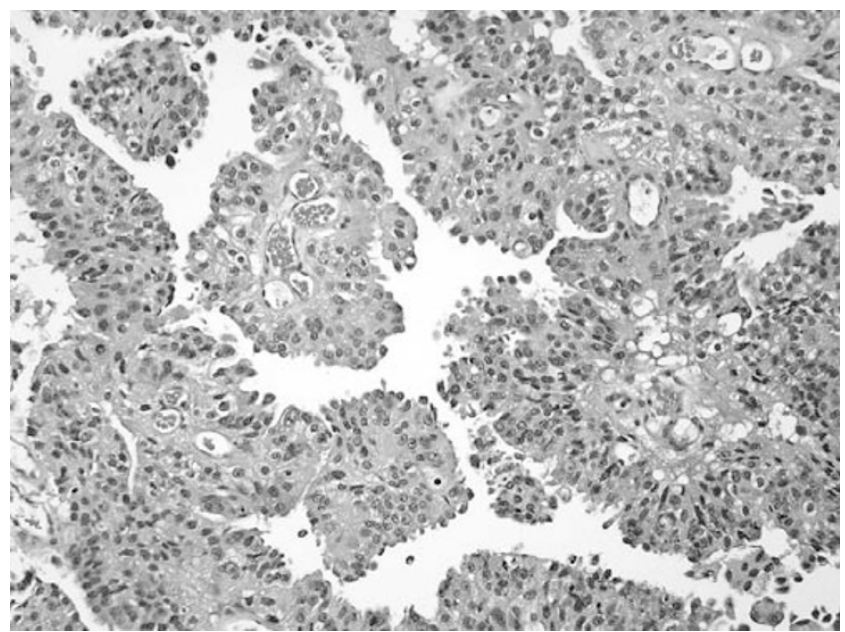

In most cases the nuclei were uniform with prominent nucleoli. 1 case had pleomorphic giant cell features. Necrosis was present in 2 cases. One case had a separate focus of low grade non-invasive UC present in the bladder as well as better differentiated PCa. (Gleason Score $4+3=7$ ) involving the prostate on needle biopsy. In all cases the pseudopapillary areas showed negative immunohistochemical staining for bladder markers including GATA3 (3 cases), p63 (3 cases), CK903 ( 2 cases), and thrombomodulin ( 3 cases). All cases showed positivity for prostatic markers including PSA ( 4 cases), p501s ( 5 cases), PSMA ( 3 cases), and NKX3.1 (3 cases). One case showed focal, non-specific staining for $\mathrm{p} 63$, contributing to the diagnostic difficulty. Conclusions: High grade PCa. can present in the urinary bladder and prostatic urethra, clinically mimicking UC. Although high grade PCa. typically has relatively uniform cytology, uncommon PCa. can have pleomorphic giant cell features overlapping with UC. Pseudopapillary features in a high grade $\mathrm{PCa}$. is a newly recognized morphological overlap that can lead to further diagnostic difficulty in distinguishing the two entities. For high grade tumors involving the prostatic urethra without typical admixed lower grade PCa., immunohistochemistry studies for bladder and prostate markers should be performed.

955 Cadherin 17 Is a Sensitive and Specific Marker for Metanephric Adenoma

Z Grada, C Magi-Galluzzi, S Mangray, R Monahan, S Lu, MB Resnick, E Yakirevich. Rhode Island Hospital and Alpert Medical School of Brown University, Providence, $\mathrm{RI}$; Cleveland Clinic, Cleveland, $\mathrm{OH}$.

Background: Metanephric adenoma (MA) is a rare benign renal neoplasm composed of small cells arranged in tightly packed acini, solid sheets, or papillary structures. Due to the morphologic similarity and overlapping immunophenotype of MA with epithelial predominant Wilms tumor (e-WT) and with solid variant of papillary renal cell carcinoma (s-PRCC) the differential diagnosis between these tumors may be challenging. Cadherin 17 (CDH17), also known as liver-intestine cadherin, is expressed primarily in the normal intestinal epithelium and digestive tract tumors and has not been detected in tumors from other sites including kidney. Our goal was to investigate the diagnostic utility of CDH17 to differentiate between MA, e-WT, and s-PRCC.

Design: Immunohistochemistry for CDH17 was performed with the SP183 rabbit MAb (1:100 dilution, Cell Marque, CA) on tissue microarrays containing $17 \mathrm{e}-\mathrm{WTs}, 15$ s-PRCCs, and 4MAs and on whole tissue sections of 14 MAs. In addition, the tumors were stained for CD57, AMACR, and WT-1. Immunoreactivity was assessed based on a combined score of extent and intensity on a scale of $0-3+$.

Results: Normal adult kidney tissues were negative for $\mathrm{CDH} 17$ staining. In the MA group $\mathrm{CDH} 17$ expression was seen in a membranous staining pattern with lateral intercellular border accentuation. CDH17 immunoreactivity was observed in all components of MAs (acinar, tubular, and papillary). The majority of MAs (78\%) demonstrated CDH17 immunoreactivity, while all cases of e-WTs and s-PRCCs were negative $(\mathrm{p}<0.0001$, table 1$)$. Ten of $14(71 \%)$ positive MAs exhibited moderate to strong $(2-3+)$ CDH17 expression. All MAs were strongly positive (3+) for CD57; however, this marker was moderate to strongly positive (2-3+) in $6(35 \%)$ e-WTs and $2(13 \%)$ s-PRCCs. AMACR was strongly $(3+)$ positive in all s-PRCCs, although moderate reactivity was seen in $3(17 \%)$ e-WTs and 1 MA $(6 \%)$. WT-1 was negative in s-PRCC and was positive in all cases of e-WT and MA.

\begin{tabular}{|c|c|c|c|c|}
\hline & $e-W T(n=17)$ & $\mathrm{s}-\mathrm{PRCC}(\mathrm{n}=15)$ & MA $(n=18)$ & P value \\
\hline CDH17 & 0 & 0 & $14(78 \%)$ & $<0.0001$ \\
\hline CD57 & $10(59 \%)$ & $3(20 \%)$ & $18(100 \%)$ & $<0.0001$ \\
\hline AMACR & $6(35 \%)$ & $15(100 \%)$ & $2(11 \%)$ & $<0.0001$ \\
\hline WT-1 & $17(100 \%)$ & 0 & $18(100 \%)$ & $<0.0001$ \\
\hline
\end{tabular}

Conclusions: $\mathrm{CDH} 17$ is a sensitive $(78 \%)$ and highly specific $(100 \%)$ marker for metanephric adenoma. CDH17 should be considered in the IHC panel for distinguishing MA from its mimics. 
956 Cellular Invasion of Fat Is an Important Prognostic Indicator in Renal Cell Carcinoma

DF Griffiths, M Varma, CR Williams, H Kynaston. Cardiff University, Cardiff, United Kingdom; Cardiff \& Vale UHB, Cardiff, United Kingdom.

Background: Most localised Renal Cell Carcinomas (RCC) are cured by surgery. However a significant minority will metastasise and lead to morbidity and mortality. It is difficult to reliably separate those cases that will metastasise from cases that require no further treatment and better prognostic models are needed. In a retrospective study concluded in 2001 we found that cellular invasion of fat, i.e. tumour cells between fat cells without intervening fibrous tissue, at the margin of the tumour indicated a significantly unfavourable prognosis. The aim of this study is to validate this prognostic covariate in a prospective series.

Design: From 1999 all 323 localised renal cell carcinomas recieved were dissected to a standard protocol and reported by 2 urological pathologists to a pro-forma that included the covariates that make up T stage, i.e. size; level of vascular invasion and whether or not cellular invasion of fat was present. Histological type, Furhman grade and necrosis were also recorded. Subsequent follow-up was determined on risk-based criteria, usually using Leibovich score. Outcome was determined by a review of the clinical notes. All cases that were localised at operation were included. Cases were censored at the latest follow-up if there was no evidence of disease or the date of death if death was due to another cause. Survival analysis (to the date of first metastasis) was by carried out by Kaplan Meier and Cox regression methods.

Results: On univariate analysis all covariates were significantly associated with prognosis $(\mathrm{P}<0.001$ by log-rank in all cases $)$. On Cox regression multivariate analysis using a forward selection procedure only grade ( grade 3 : hazard ratio $(\mathrm{HR})=3, \mathrm{p}=0.0002$; grade 4: $\mathrm{HR}=6.8, \mathrm{p}<0.0001)$, cellular invasion of fat $(\mathrm{HR}=8.0, \mathrm{P}<0.0001)$ and necrosis $(\mathrm{HR}=2.0, \mathrm{P}=0.025)$ contributed to the outcome. Tumour type, size and vascular invasion were rejected by the selection procedure.

Conclusions: Cellular invasion of fat appears to be an important prognostic determinant in renal cell carcinoma and confirms the findings in our previous study. Separating the elements that make up stage is likely to have advantages in developing prognostic models as these covariates are genuinely independent and may each have influence on outcome. Our study suggests the main independent covariates influencing outcome are grade, cellular invasion of fat and necrosis. This does not exclude vascular invasion, size or histological type having influence on the outcome; however a larger series may be required to determine their importance.

957 Which Method of Measuring Tumour Length in Prostate Biopsies Best Predicts Clinically Significant Cancer?

DF Griffiths, $M$ Varma, $R$ Parry, DLL Cochlin. Cardiff University, Cardiff, United Kingdom; Cardiff \& Vale UHB, Cardiff, United Kingdom.

Background: Morphometric theory predicts, and practical experience confirms, that the amount of tumour in protocol prostatic needle biopsy cores correlates with the tumour volume in a subsequent radical prostatectomy (RP). Tumour volume correlates with both Grade and Stage. Core measurements are thus often valuable in subsequent management decisions. However there is no agreed way to measure tumour length in a core. There are theoretical reasons for using the dimension between the outer margins of tumour in the core involved - [the whole tumour length (WTL)]. Some pathologists are reluctant to report two small separate foci as a single long length and prefer to sum the lengths of separate foci - [the sum tumour length (STL)]. The aim of the study is to determine the measurement which best predicts tumour size, and hence gives the most appropriate information to clinicians.

Design: Patients who had prostate biopsies taken by two urological radiologists to a standard protocol that were found to have only one core positive and then went on to have a RP were identified. The length of tumour in the single core was measured by the two methods (WTL and STL). The volume of tumour in the subsequent RP was measured by a standard method. The discriminating power of the different length measurements for identifying significant tumours (defined as tumours $>0.5 \mathrm{ml}$ volume) was tested by the receiver operating characteristic (ROC).

Results: 113 of a series of 661 RPs had a single core positive in prior protocol biopsies There was a difference between the two tumour measurements in the cores of at least $1.0 \mathrm{~mm}$ in 18 of the 113 cases. The length measured by both methods strongly correlated with subsequent tumour volume. The ROC the area under the curve was 0.61 for the STL and 0.67 for the WTL. Both measurements were significantly different from 0.5 (no discriminating power) however the WTL was the better discriminator at all thresholds $(\mathrm{p}=0.049$ for STL; $\mathrm{P}=0.003$ for WTL).

Conclusions: WTL is a simpler method and is a better predictor of subsequent tumour volume. It is thus recommended as the method used to report tumour length in prostate cores. This length should also be used when calculating percentage involvement of cores by tumour.

958 Tuberous Sclerosis Complex (TSC)-Associated Renal Cell Carcinoma (RCC): A Clinicopathologic Study of 27 Tumors in 15 Patients $J$ Guo, MS Tretiakova, ML Troxell, AO Osunkoya, O Fadare, AR Sangoi, S Shen, $R$ Mehra, A Heider, JP Higgins, A Lopez-Beltran, C Przybycin, C Magi-Galluzzi, JK McKenney. Cleveland Clinic, Cleveland, $\mathrm{OH}$; University of Washington, Seattle, WA; OHSU, Portland, OR; Emory University, Atlanta, GA; Vanderbilt University, Nashville, TN; El Camino Hospital, Mountain View, CA; Houston Methodist Hospital, Houston, TX; University of Cordoba, Cordoba, Spain; University of Michigan, Ann Arbor, MI; Stanford, Stanford, CA

Background: Angiomyolipoma (AML) is common in TSC, but RCC is only rarely reported. We describe a clinicopathologic series of TSC-associated RCCs.
Design: 15 patients with RCC and clinical history of TSC were collected from multiple institutions. All cases were re-reviewed. Standard clinical data, detailed histologic features, and immunohistochemical findings (CK7, CA9, CD117, PAX8) were analyzed. Results: 27 RCCs from 15 TSC patients ( 11 female and 4 male) were reviewed. Age ranged from 7-65 years (mean: 42 ). 8 patients (53\%) had multiple RCCs (range: 2-6 tumors). 13 patients $(87 \%)$ had concurrent renal AMLs ( 8 with epithelial cysts). Background kidney contained cysts in 9 patients $(60 \%)$ and 8 patients $(53 \%)$ had intratubular epithelial changes (cytoplasmic eosinophilia, nucleomegaly, and prominent nucleoli). 3 morphologic patterns were seen: 10 (37\%) had features identical to a subset described previously as "renal angiomyoadenomatous tumor" (RAT-like pattern); 12 (44\%) had chromophobe RCC-like morphology; 5 (19\%) had a previously described unique eosinophilic and cystic morphology. 12 cases had material for immunohistochemistry. All RCCs tested were PAX8 positive. 3 of 4 RAT-like tumors showed strong and diffuse reactivity for CA9 and CK7, while focal and weak positive staining was seen in 1 . All 5 chromophobe RCC-like were positive for CK7; only 1 of 5 expressed CD117. Of the 3 RCCs with eosinophilic-cystic morphology, one was CK7 positive and CD117 negative, while 2 were both CK7 and CD117 negative. Clinical follow-up data was available in 10 patients; none had evidence of disease at last follow-up (range: 11-198 months; mean: 58). One patient with RAT-like RCC had metastatic RCC in 2 regional lymph nodes at nephrectomy, but is currently disease free at 32 months.

Conclusions: TSC-associated RCCs had 3 distinct histologic patterns, commonly had eosinophilic epithelial changes in the background kidney, and had a propensity for multiple tumors. Knowledge of these pathologic findings may allow better recognition of TSC in kidney resection specimens.

959 Proximity of the Tumour in Extraprostatic Location to the Resection Margin: Is There Prognostic Significance?

$R$ Gupta, $R$ O'Conell, A-M Haynes, PD Stricker, W Barett, JJ Turner, W Delprado, $L G$ Horvath, JG Kench. Royal Prince Alfred Hospital, Sydney, Australia; University of Sydney, Sydney, Australia; The Kinghorn Cancer Center, Sydney, Australia; St. Vincent's Hospital, Sydney, Australia; Douglass Hanly Moir Pathology, Sydney, Australia.

Background: A surgical margin of radical prostatectomy is considered negative if the carcinoma does not reach the inked resection margin even with microscopic clearance of $<0.1 \mathrm{~mm}$ (ISUP 2009). While the fibromuscular prostatic stroma may act as a limiting barrier for organ confined prostate cancer, it is lacking in tumours with extraprostatic extension (EPE). Positive surgical margin in EPE is associated with biochemical (BCR) and clinical relapse (CR); however, the prognostic significance of proximity of EPE tumour to the margin is not known.

Design: Patients with EPE (ISUP 2011) without positive surgical margins, seminal vesicle or lymph node involvement were identified from a radical prostatectomy cohort of 1136 (2002-2006) and analysed for 1)proximity of the EPE tumour from the margin inmm, 2)extent of EPE, 3) Gleason score of the tumour in EPE. Log-rank, Kaplan-Meier, Cox regression analyses were done.

Results: The study includes 194 cases with a median follow up of $5.4 y$ with $37(20 \%)$ BCRs and $6(3.1 \%)$ CRs. The distance of the EPE tumour from the margin ranged from $0.01 \mathrm{~mm}-7.5 \mathrm{~mm}$. The proximity to the margin did not correlate with BCR or CR (HR:0.69; 95\%CI:0.19-2.47) with 5y survival rates of 82 and $91 \%$ respectively for patients with EPE tumour reaching $<0.1 \mathrm{~mm}$ and those $\geq 0.1 \mathrm{~mm}$. On univariate analysis patients with EPE Gleason score 8 and higher showed increased incidence of BCR as compared to those with EPE Gleason scores of 6 or $7(p=0.021)$. On multivariate analysis, adjuvant therapy was the only factor that correlated with BCR $(p=0.0005)$. The Gleason score of the entire prostatectomy specimen, of the EPE tumour or the extent of EPE did not correlate with BCR in pT3aN0R0 cohort.

Conclusions: This study includes a cohort of patients (pT3aR0N0)that is frequently seen in routine practice. Data from 194 patients with a median follow up of $5.4 \mathrm{y}$ using current EPE criteria and Gleason scores show that proximity of tumour in EPE to the margin is not prognostically significant. Gleason scores of the EPE tumour carry prognostic significance on univariate analysis. Larger studies are needed to study whether extensive Gleason pattern 4 in an EPE indicates increased risk in an otherwise Gleason score 7 tumour.

960 Nested and Microcystic Variants of Urothelial Carcinoma Displaying Immunohistochemical Features of Basal-Like Urothelial Cells: An Immunohistochemical and Histopathogenetic Study of Rare Variants of Urothelial Carcinoma Including Large Nested Variant, Plasmacytoid Variant and Inverted Papilloma

SW Hakim, PY Gulavita, CG Ball, B Djordjevic, SJ Robertson, Z Kos, TA Flood, EC Belanger, KT Mai. University of Ottawa, Ottawa, ON, Canada.

Background: Nested/microcystic (NV/MV) and plasmacytoid (PV) urothelial carcinoma (UC) variants are low grade neoplasms but commonly high-stage disease processes at diagnosis. Their histopathogenesis, along with that of large nest variant (LNV) and inverted papilloma (IP), are not fully understood. We hypothesize that these rare variants of UC may represent basal-like urothelial carcinoma (BUC) and investigate using urothelial basal/stem cell markers.

Design: Cases of 14 NV/MV, 8 PV, 3 LNV and 5 IP were retrieved from our files. Representative sections were stained with CD44, CK5, p63, CK34bE12, bcl2 and EP4. Immunoreactivity was scored as 0 (negative), 1 (focal staining in the basal layer or peripheral cells), 2 (intermediate; between 1 and 3 ) and 3 (moderate to marked reactivity for $\mathrm{CK} 5$,or mild to marked reactivity for CD44 with: a) full or more than 50\% thickness of the epithelial cell nest, in areas totalling at least $3 \mathrm{~mm}$ in diameter, or b) more than $50 \%$ of the invasive component in areas totalling less than $3 \mathrm{~mm}$ in diameter). Invasive 
carcinoma and neoplastic surface urothelium with a score of 3 were designated as BUC and basal-like intra-urothelial neoplasia, respectively. Ten cases of high grade urothelial carcinoma of the usual type were used as controls.

Results: $14 / 14$ NV/MV, 3/8 PV, 1/3 LNV and 5/5 IP showed extensive immunoreactivity (score 3) for CK5, CD44 and CK34bE12; while the tumor nest peripheral cells showed negative, or focal bcl2/EP4 reactivity. The control cases, while often strongly reactive for CK34bE12, were focally reactive or negative for CK5, CD44, p63, bc12 and EP4. Conclusions: NV/MV specifically immunostain for CK5 and CD44, contrasting other seemingly non-specific or non-sensitive markers. NV/MV represent immunohistochemical variants with basal-like cell features with aggressive behavior similar to basal-like breast carcinoma and non-cutaneous basaloid squamous cell carcinomas.

961 Two Consecutive Atypical Glands in Prostate Needle Core Biopsy: Clinical Follow-Up Data from a Large Multicenter Clinical Experience

JS Han, KD Jewell. Kaiser Permanente Oakland Medical Center, Oakland, CA.

Background: There is little follow up data for patients with atypical glands, suspicious for carcinoma in two consecutive needle core biopsies of the prostate.

Design: From 17 Kaiser Permanente Medical Centers in the Northern California region, the pathology database was searched between 2006-2009 for two consecutive prostate needle core biopsies (PNCBs) with the diagnosis of atypical glands. Patients with a prior diagnosis of prostate cancer were excluded. Data regarding follow up biopsies, PSA and PSA density (PSAD) were obtained.

Results: Out of 27,256 PNCBs during the period, 100 men with two consecutive atypical diagnoses were identified. Sixty six (66) men had at least one additional biopsy. 18 of $66(27 \%)$ men showed carcinoma on the third biopsy. 11 additional men were diagnosed with carcinoma during the follow up period (ranging from the 4th-6th total biopsy sets), resulting a total of 29/66 (43.9\%) men with carcinoma. The time between the first atypical diagnosis and the final cancer diagnosis ranged between 3 - 62 months with a median of 28 months. No significant differences in PSA or PSAD were present between men with carcinoma and men without a carcinoma diagnosis. Different PSA cutoff values failed to demonstrate predictability for final cancer diagnosis.

\begin{tabular}{|l|l|l|}
\hline & 3rd Biopsy & Last biopsy (4th-6th) \\
\hline Benign & $32(48.5 \%)$ & $27(40.9 \%)$ \\
\hline PIN & $2(3 \%)$ & $3(4.5 \%)$ \\
\hline Atypical glands & $14(21.2 \%)$ & $7(10.6 \%)$ \\
\hline Carcinoma & $18(27.3 \%)$ & $29(43.9 \%)$ \\
\hline
\end{tabular}

Conclusions: After two consecutive atypical diagnoses on $\mathrm{PNCB}$, the probability of carcinoma diagnosis on third biopsy appears to be slightly lower than the reported risk of carcinoma in the literature (approximately $40 \%$ ) after one atypical diagnosis. However, a significant number of these patients were diagnosed with carcinoma in subsequent biopsies. PSA or PSAD are not predictive of biopsy findings on these patients.

962 Differential Analysis of HNF1 $\beta$ IHC Expression in Metanephric-Derived Tissues and Neoplasms

MG Hanna, N Patil, P Unger. Icahn School of Medicine at Mount Sinai/The Mount Sinai Hospital, New York, NY.

Background: The Hepatocyte nuclear factor (HNF) family of transcription factors is partly involved in liver and kidney development. HNF1 $\beta$ is particularly involved in genitourinary organogenesis. While HNF $1 \beta$ expression has been studied in ovarian clear cell carcinomas, there is only scarce literature on its expression in various metanephric-derived tissues and proliferations with clear cells, with proposed role in tumorigenesis. We aim to report our experience with $\mathrm{HNF} 1 ß$ expression in the subtypes of renal cell carcinoma (clear cell, papillary, and chromophobe), nephrogenic adenomas, oncocytomas and clear cell carcinomas of the lower genitourinary tract.

Design: Paraffin-embedded, formalin-fixed blocks representing various metanephric tissues were randomly selected from the Pathology archives. HNF $1 \beta$ IHC expression ( $1: 200$, rabbit polyclonal $\mathrm{Ab}$ ) was read by two pathologists, and strong nuclear staining was interpreted as positive; cytoplasmic staining was disregarded. Staining of renal tubules and ovarian clear cell carcinomas were used for comparison.

Results: In our series, strong diffuse HNF $1 \beta$ positivity was seen in all clear cell RCCs $(11 / 11 ; 100 \%)$, papillary RCCs $(6 / 6 ; 100 \%)$, lower GU nephrogenic adenomas $(6 / 6$; $100 \%$ ), and lower GU tract clear cell carcinomas $(3 / 3 ; 100 \%)$. Chromophobe renal carcinomas $(0 / 6 ; 0 \%)$ and oncocytomas $(0 / 4 ; 0 \%)$ showed lack of HNF1 $\beta$ staining. Strong positive staining was seen in $26 / 26$ non-neoplastic proximal and distal tubules, and parietal layer of Bowman capsules; visceral layer of Bowman capsules stained negative.

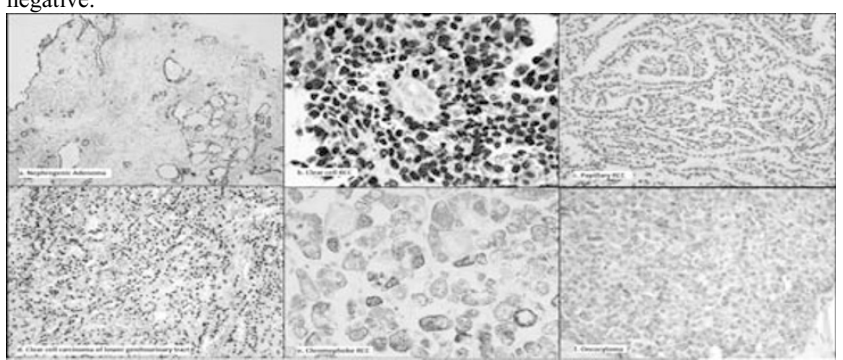

Conclusions: 1 . Our study reports on differential HNF $1 \beta$ expression in metanephricorigin tissues, including normal tubules, proliferations and tumors.

2. All Chromophobe RCCs and oncocytomas showed lack of nuclear staining with HNF $1 \beta$ in our series. This is in contrast to previous report by Wang et al (2013) who found positivity in oncocytomas. Based on their findings, they had recommended use of HNF $1 \beta$ in the differential diagnosis of oncocytoma vs Chromophobe RCC. Based on our experience, we cannot recommend use of HNF $1 \beta$ in this differential.

3. All lower GU nephrogenic adenomas stained with $\mathrm{HNF} 1 \beta$, supporting their origin from renal tubular cells rather than a metaplastic phenomenon as was previously believed.

963 TMPRSS2-ERG Type VI Isoform Is Associated with a Higher Quantitative mRNA Expression of the TMPRSS2-ERG Rearrangement in Prostatic Adenocarcinoma

$S$ Hernandez, A Font, S de Muga, $N$ Juanpere, L Agell, $M$ Lorenzo, JA Lorente, $O$ Arango, S Serrano, J Lloreta. Universitat Pompeu Fabra, Barcelona, Spain; Hospital del Mar-Parc de Salut Mar-IMIM, Barcelona, Spain; Autonomous University of Barcelona, Barcelona, Spain.

Background: Many different types of TMPRSS2-ERG fusion transcripts, involving several exons of TMPRSS 2 and $E R G$ are found in prostate cancer $(\mathrm{PCa})$. The most frequent variants involve TMPRSS2 exon 1 (T1) or 2 (T2) fused to $E R G$ exons 2 (E2), 3 (E3), 4 (E4) or 5 (E5). Type III (T1/E4) is the most frequently expressed isoform in PCa, either alone or in combination with other variants. Type VI (T2/E4) is the second most frequent and is associated with more aggressive tumors. The aim of this study has been to investigate the role of the different TMPRSS2-ERG rearrangement variants in PCa. Design: RNA was extracted in $64 \mathrm{PrCa}$ and 3 non-carcinomatous prostate tissues (Parc de Salut MAR Biobank, Barcelona, Spain). TMPRSS2-ERG and ERG mRNA expression was analyzed by qRT-PCR (Applied Biosystems, Foster City, CA, USA). The cDNA from the quantitatively positive TMPRSS2-ERG cases was, in addition, amplified by RT-PCR with a forward primer located in TMPRSS2 exon 1, and a reverse located in $E R G$ exon 5. PCR products were purified and sequenced with the Big Dye Terminator Kit v.3.1 (ABIPRISM 377, PerkinElmer Applied Biosystems).

Results: TMPSS2-ERG and ERG mRNA were overexpressed in 38 and 40 prostate tumors, respectively. The 38 cases positive for the TMPRSS2-ERG rearrangement were sequenced. In $60.5 \%$ of these cases, only variant III was detected, and in $39.5 \%$ of cases two variants were detected: variant III+VI in $36.8 \%$ and variant III+I (T1/E2) in $2.6 \%$. Quantitative expression of TMPRSS2-ERG was higher in tumors with variants III+VI than in tumors with only variant III (Pearson Chi-Square $\mathrm{p}=0.021$ ). Also levels of $E R G$ mRNA were higher in tumors with variants III+VI or I than in tumors with only variant III, but this difference was not statistically significant. Variant III alone was more prevalent in prostate tumors with Gleason sum $\leq 7$, and variant III+VI was more prevalent in prostate tumors with Gleason sum $\geq 8$. However this difference was not statistically significant.

Conclusions: TMPRSS2-ERG variant III+VI is associated with higher TMPRSS2-ERG mRNA expression, but not with higher $E R G$ mRNA expression, than variant III alone. Variant VI is more prevalent in high grade prostate tumors.

Supported by FIS/ Instituto Carlos III/ FEDER PI12-01426, Spanish Ministry of Health.

964 High Levels of TMPRSS2-ERG and ERG mRNA, and Loss of Expression of PTEN Are More Frequent in High Gleason Score Prostate Tumors

$S$ Hernandez, S de Muga, A Font, L Agell, N Juanpere, M Lorenzo, JA Lorente, $S$ Serrano, J Lloreta. Pompeu Fabra University, Barcelona, Spain; Hospital del Mar, Barcelona, Spain; Hospital del Mar-IMIM, Barcelona, Spain; Autonomous University of Barcelona, Barcelona, Spain.

Background: TMPRSS2-ERG fusion is the most common alteration leading to $E R G$ mRNA overexpression and ERG protein acumulation in prostate cancer (PCa). There is controversy on its prognostic implications. ERG genetic rearrangements and PTEN loss are reported as frequent, often concomitant events that may have a cooperative role in PCa progression. The aim of this study is to investigate the quantitative mRNA levels of TMPRSS2-ERG, ERG, and PTEN in PCa and its relationship with ERG protein histoscore and combined Gleason score.

Design: RNA was extracted in $64 \mathrm{PCa}$ and 3 non-neoplastic prostate tissues (Parc de Salut MAR Biobank, Barcelona, Spain). TMPRSS2-ERG (Hs03063375_ft), ERG (Hs01554629_m1) and PTEN (Hs02621230_s1) mRNA expression was analyzed by qRT-PCR (Applied Biosystems, Foster City, CA, USA). GADPH (4310884E) was the internal control. ERG immunostaining (EPR3864, Epitomics, Burlingame, CA, USA) was done in $58 \mathrm{PCa}$, and quantitated with a combined intensity and percentage histoscore.

Results: Thirty-eight (59.3\%) and 40 (62\%) PCa overexpressed TMPSS2-ERG and $E R G$ mRNA, respectively. Quantitative levels of TMPRSS2-ERG and $E R G$ showed a statistical correlation (Spearman's rho coefficient correlation $=0.821, \mathrm{p}<0.0001$ ). There is a very good concordance between positive/negative TMPRSS2-ERG and ERG mRNA overexpression with ERG immunostaining (Pearson Chi-Square $<0.0001$ ). However, the different levels of quantitative TMPRSS2-ERG and ERG mRNA, and ERG IHQ histoscore were not related. Twenty-one PCa (40.4\%) had loss of PTEN expression. Decreased PTEN expression is more frequent in PCa prostate tumors with TMPRSS2$E R G$ (Pearson Chi-Square, $\mathrm{p}=0.006$ ) and $E R G$ (Pearson Chi-Square, $\mathrm{p}=0.003$ ) mRNA overexpression. There is also decreased PTEN expression related to ERG positive immunostaining (Fisher's Exact Test, $\mathrm{p}=0.006$ ). Loss of PTEN mRNA expression, alone (Pearson Chi-Square $=0.032$ ) or concurrent with TMPRSS2-ERG (Fisher's Exact Test, $\mathrm{p}=0.036$ ) and $E R G$ (Fisher's Exact Test, $\mathrm{p}=0.031$ ) mRNA overexpression are associated with Gleason $\operatorname{sum} \geq 7$.

Conclusions: Decreased PTEN expression alone or concurrent with TMPRSS2-ERG and $E R G$ mRNA overexpression are associated with high grade PCa and could be molecular markers of prostate tumor progression. More studies are needed to clarify their exact role in prostatic carcinogenesis.

Supported by FIS/ Instituto Carlos III/ FEDER PI12-01426, Spanish Ministry of Health. 


\section{mRNA Overexpression}

S Hernandez, A Font, N Juanpere, S de Muga, LAgell, MLorenzo, JA Lorente, S Serrano, J Lloreta. Universitat Pompeu Fabra, Barcelona, Spain; Hospital del Mar-Parc de Salut Mar-IMIM, Barcelona, Spain; Autonomous University of Barcelona, Barcelona, Spain. Background: Complex intra- and inter- chromosomal rearrangements in TMPRSS2 and $E R G$ are a common and specific feature in prostate cancer $(\mathrm{PCa})$. In addition to $E R G$, TMPRSS 2 is less often fused to other ETS members such as ETV1, ETV4 and ETV5. Other less frequent 5' partners such as $S L C 45 A 3$ and $N D R G$ have also been identified. The aim of the present study has been to investigate the effect of the SLC45A3-ERG rearrangement on the quantitative $E R G$ expression.

Design: RNA was extracted in $64 \mathrm{PCa}$ and 3 non-neoplastic prostate samples (Parc de Salut MAR Biobank, Barcelona, Spain). ERG mRNA expression was analyzed by quantitative RT-PCR (Hs01554629_m1) (Applied Biosystems, Foster City, CA, USA). GADPH (4310884E) was used as internal control. SLC45A3-ERG rearrangement mRNA expression was analyzed by RT-PCR with a forward primer located in SLC45A3 exon 1 "AACCAGCCTGCACGCGCTGG" and a reverse located in ERG exon 5 "GGCGGGAAGATGGTGGGCAG". PCR products were separated by electrophoresis and visualized with ethidium bromide. Sequencing analysis was performed with the Big Dye Terminator Kit v.3.1 (ABIPRISM 377, PerkinElmer Applied Biosystems). Results: The mean basal $E R G$ expression level in the non-neoplastic prostate samples was 0.029 . Levels $\geq 0.13\left(2^{\wedge}(-\mathrm{DCt})\right)$ were considered overexpression. A total of 40 tumors $(62 \%)$ overexpressed $E R G$ mRNA: low overexpression ( +1$)$ from 0.13 to 0.53 $(\mathrm{n}=11)$, intermediate $(+2) 0.54$ to $0.97(\mathrm{n}=12)$, and strong $(+3) \geq 1(\mathrm{n}=17)$. SLC45A3$E R G$ rearrangement expression was considered positive when we detected a visible band in the agarose gel. Fifteen of 63 (24\%) PCa expressed SLC45A3-ERG. Six of them had low levels $(+1), 7$ intermediate levels $(+2)$, and only in 2 PCa high levels of $S L C 45 A 3-E R G$ mRNA (+3) were found. There was no relationship of this rearrangement (presence or absence; quantitative levels) with quantitative $E R G$ mRNA expression. The 15 cases with $S L C 45 A-E R G$ rearrangement were sequenced. All presented the same type of rearrangement between exon 1 of SLC45A3 and exon 4 of $E R G$

Conclusions: There is no relationship between presence or absence of $S L C 45 A-E R G$ rearrangement, nor between the different levels of this fusion, and quantitative $E R G$ mRNA expression. The 5' partner $S L C 45 A 3$ does not seem to be as powerful as $T M P R S S 2$ in the activation of $E R G$ transcription.

Supported by FIS/ Instituto Carlos III/ FEDER PI12-01426, Spanish Ministry of Health.

\section{Choriogonadotropin Positive Seminomas-Clinicopathological and} Molecular Genetic Analysis of $\mathbf{1 5}$ Cases

O Hes, K Pivovarcikova, J Stehlik, $M$ Hora, M Michal. Charles University, Medical Faculty and Charles University Hospital, Plzen, Czech Republic.

Background: Human choriogonadotropin (hCG) positive syncitiotrophoblastic and/ or large cells (STC) present within classic seminoma (CS) are well documented. A finding of CS with extensive hCG positive areas without STC is rare. In this study, we present 15 such cases.

Design: 168 CSs were retrieved from Plzen Tumor registry. The study excludes mixed germ cell tumors and CSs with STC. Cases with a completely embedded tumorous mass were selected for further study and stained with hCG. Positive cases were further analyzed by RT PCR

Results: 2 groups of hCG positive CSs were identified. Group 1: 10 cases, mean patient age was 37.7 years, mean tumor size was $4.96 \mathrm{~cm}$. 8 cases were pT1 (TMN 2009), 2 cases pT3a. Blood level of hCG was elevated in 6/10 patients preoperatively. In 2 patients blood level was not tested. Mean follow up period was 6.1 years. No metastatic behavior was noted. All tumors were extensively immunoreactive for hCG in more than $60 \%$ of tumorous tissue. Expression of hCG beta subunit (CGB) in tumor tissue was also observed at mRNA level. Group 2: 5 cases, mean age was 34 years, mean size was $4.7 \mathrm{~cm}$. 4 cases were pT1, 1 case was pT2 stage. Mean follow up period was 2.5 years. No metastatic behavior was noted. Preoperative blood level of hCG was elevated in $1 / 5$ patient. Strong hCG positivity was limited to scattered single tumorous cells distributed through the entire tumor. Only weak expression of CGB mRNA was detected in tumor tissue.

Conclusions: 1 . Immunohistochemical hCG positivity in CSs is not limited to the syncitiotrophoblastic or large mono-multinucleated cells.

2. 2 basic immunohistochemical patterns could be seen in hCG positive CSs: a) extensive positivity of tumorous tissue. B) scattered single strongly hCG positive cells present within tumor.

967 Distinctive Renal Cell Tumor Simulating Atrophic Kidney with Two Types of Microcalcifications. Report of Three Cases

O Hes, TG de Souza, L Straka, M Svajdler, M Hora, M Michal. Charles University Hospital and Medical Faculty, Plzen, Czech Republic; Hospital Alianca, Sao Paulo, Brazil; Klinicka Patologia, Presov, Slovakia (Slovak Republic); Pasteur University Hospital, Kosice, Slovakia (Slovak Republic).

Background: We report 3 cases of primary renal cell tumor simulating atrophic kidney with distinct gross, morphologic, immunohistochemical, and molecular genetic features. Design: The tumors were retrieved out of more than 17000 renal tumors from the Plzen Tumor Registry. Tissues for light microscopy had been fixed, embedded and stained with hematoxylin and eosin using routine procedures. The tumors were further analyzed using immunohistochemistry, arrayCGH, and HUMARA. Analyses of $V H L$ gene and LOH3p were also performed.

Results: The patients were 2 females and 1 male, with age range 29-35 years (mean 31.3 years). Grossly, the neoplasms were encapsulated, round with largest diameter $3.5 \mathrm{~cm}$ (mean $3.2 \mathrm{~cm}$ ). Follow up available for all patients ranged from 2 to 14 years (mean 8 years). No aggressive behavior was noted. Histologically, akin atrophic (postpyelonephritic) kidney parenchyma, the tumors were composed of follicles of varying sizes that were filled by eosinophilic secretion. Rare areas contained collapsed follicles. Each follicle was endowed with a small capillary. The stroma was loose, inconspicuous and focally fibrotic. Two types of calcifications were noted: typical psammoma bodies and amorphous dark blue stained calcified deposits. Immunohistochemically, tumors were strongly positive for cytokeratins (OSCAR), CD10 and vimentin, with weak immunopositivity for CAM5.2 and AE1-AE3. WT1 and cathepsin $\mathrm{K}$ were weakly to moderately focally to diffusely positive. Tumors were negative for CK 20, carbonic anhydrase IX, parvalbumin, HMB45, TTF1, TFE3, chromogranin A, thyroglobulin, PAX8, and ALK. Only 1 case was suitable for molecular genetic analyses. No mutations were found in the $V H L$ gene; no methylation of $V H L$ promoter was noted. No numerical aberrations were found by aCGH analysis. LOH for chromosome $3 p$ was not detected. Analysis of clonality (HUMARA) revealed monoclonal nature of the tumor.

Conclusions: We describe unknown tumor of the kidney which: 1) resembles renal atrophic kidney or nodular goiter of thyroidal gland; 2) contains a leiomyomatous capsule and two types of calcifications; 3 ) lacks mitoses, atypias, necroses, hemorrhages; nearly lack Ki-67 positivity; 4) so far showed benign biological behavior.

\section{Major Histocompatibility Complex Class I Expression in Clear Cell} Renal Cell Carcinoma: Correlation with Clinical Outcome

SA Holzman, BP Pollack, VA Master, AO Osunkoya. Emory University School of Medicine, Atlanta, GA.

Background: Majority of patients with clear cell renal cell carcinoma (CCRCC) initially present with localized disease with the potential for curative nephrectomy, however one-third subsequently develop metastatic disease. Current predictive models of CCRCC recurrence are suboptimal, thus there is a need to develop better predictors of metastasis. CCRCC is an immunogenic malignancy, suggesting that the immune system plays a role in tumor progression. In this study, we analyzed major histocompatibility complex class I (MHCI) expression in CCRCC with emphasis on correlation with clinical outcome. Design: A search was made through our files for patients with CCRCC that had radical nephrectomy as monotherapy, and also had at least four years of follow-up. Patients with pT4 disease or metastasis at initial presentation were excluded. All the slides were re-reviewed by a Urologic Pathologist, and a block with tumor was selected from each case. Immunohistochemical staining for MHCI was performed. We utilized whole slide scanning and automated image analysis; representative areas of tumor and normal kidney were selected and averaged using Aperio image analysis software (positive pixel count version 9). Unpaired t-test and one-way ANOVA were performed with GraphPad Prism. Results: In this pilot study, 34 patients that met our criteria were selected for analysis. Mean patient age was 64 years (range: $48-89$ years). Mean tumor size was $7.2 \mathrm{~cm}$ (range: $2.5-15.2 \mathrm{~cm}$ ). Fuhrman nuclear grades (FNG) were as follows; FNG2 10/34 patients (29\%), FNG 3 20/34 (59\%) patients and FNG 4 4/34 (12\%) patients. Although there was no statistically significant correlation with Fuhrman nuclear grade (ANOVA, $\mathrm{p}=0.800$ ), patients who were alive at follow up had increased MHCI expression $(80.1 \%$ average positivity score) than those who died of disease (53\% average positivity score; t-test, $\mathrm{p}<0.0001)$. In addition, patients who were alive with recurrence had increased MHCI expression ( $81.3 \%$ positivity score) compared to those who succumbed to their disease recurrence $(53.2 \%$ positivity score; $t$-test, $\mathrm{p}<0.0001)$.

Conclusions: This study demonstrates that MHCI may be an important prognostic factor in CCRCC for both recurrence free survival and for patients with recurrence. This is also the first study to demonstrate that increased MHCI expression is a favorable prognostic indicator in patients with metastatic CCRCC. Additionally, our results suggest that MHCI plays an important role in tumor-host immune system interaction in patients with CCRCC, and deserves further investigation

969 Many Postchemotherapy Sarcomatous Tumors in Patients with Testicular Germ Cell Tumors (TGCT) Are Sarcomatoid Yolk Sac Tumors (SYST): A Study of $\mathbf{3 6}$ Cases

BE Howitt, M Magers, K Rice, C Cole, TM Ulbright. Brigham and Women's Hospital, Boston, MA; Indiana University School of Medicine, Indianapolis, IN.

Background: Sarcomatoid neoplasms in patients with TGCTs may show diverse lineages and are usually attributed to "transformation" of teratoma, although origin from YST has also been suggested. We studied sarcomatoid tumors with non-specific morphology (NOS) in TGCT patients that had immunohistochemical (IHC) properties of YST.

Design: 36 sarcomatoid tumors NOS from 23 TGCT patients were reviewed, immunostained and correlated with clinical data. Evaluated features were: atypia (mild, moderate, severe), cellularity, tumor necrosis, mitotic rate, stromal vascularity, cell profile (spindle or epithelioid) and stroma (myxoid and/or fibrous). IHC stains were: AE1/AE3, SALL4, glypican3 (GPC3), AFP, p63, GFAP, CD34, MUC4, SMA, desmin, caldesmon, and myogenin. Staining intensity $(0=$ negative, $1=$ weak, $2=$ moderate, $3=$ strong $)$ and extent $(0=<1 \%, 1=1-10 \%, 2=10-50 \%, 3=>50 \%)$ were scored. Tumors with at least moderate intensity and $>10 \%(+)$ cells for both AE1/AE3 and GPC3 were considered to be SYST. Tumors were graded based on the French sarcoma grading system, with grades 2-3 considered high grade (HG)

Results: Of the 36 cases, 22 (14 patients, age 18-38 y, mean, 27) met the IHC criteria for SYST, all occurring post-chemotherapy (mean $71 \mathrm{~m}$ after TGCT diagnosis; range $3-132 \mathrm{~m})$. SYST had spindled (100\%; 3 focal) and epithelioid cells $(77 \% ; 1$ focal and 3 predominant) in myxoid to fibrous stroma. 13/22 exhibited at least focally severe nuclear atypia. Distinctive tumor "ringlets" and parietal YST differentiation were common. All, by definition, were (+) for AE1/AE3 and GPC3. Additionally, 15/22 cases were SALL4(+); 10/22 had at least focal CD34 (+); and 2/22 showed focal p63 $(+) .50 \%$ exhibited smooth muscle differentiation as evidenced by desmin $(8 / 19)$, 
caldesmon (2/4), and/or SMA (4/6). AFP, MUC4, GFAP, and myogenin were (-) in all cases. On followup, 8/14 died of disease (DOD; mean $58 \mathrm{~m}$; range, 7 to $217 \mathrm{~m}$ after initial SYST diagnosis) while $5 / 14$ were alive and had no evidence of disease at last followup (ANED; mean $83 \mathrm{~m}$; range 1 - $259 \mathrm{~m}$ ). One patient died of unrelated causes (DOC) $39 \mathrm{~m}$ after SYST diagnosis. Of 11 patients with HG tumors, 8 DOD, 1 DOC, and 2 ANED, and 3 patients with grade 1 tumors were ANED.

Conclusions: A high proportion of sarcomatoid tumors in postchemotherapy resections of TGCT patients are SYSTs. These typically occur several years after diagnosis, often show myogenic differentiation and behave aggressively when $\mathrm{HG}$.

970 Validating AGR2 Protein as a Potential Prostate Cancer Biomarker $R$ Hu, S Drew, W Huang. University of Wisconsin-Madison, Madison, WI. Background: AGR2 protein, a human orthologue of the secreted Xenopus Laevis anterior gradient protein XAG-2, is overexpressed in multiple human carcinomas. Overexpression of AGR2 was observed in prostate cancer cells, and AGR2 has been explored as a potential blood based biomarker for metastatic prostate cancer. However, the association between AGR2 expression and various prostate cancer clinicopathological parameters in the limited studies was controversial. This is the first quantitative study using a high density tissue microarray (TMA) and automated morphometric technology.

Design: AGR2 monoclonal antibody was provided by Dr. Jun Luo (Johns Hopkins University). A prostate tissue microarray (pTMA) consisting of 384 duplicate cores from prostate cancers of different stages and Gleason scores was previously constructed. The pTMA was double-stained with E-cadherin, AGR2 antibodies and visualized with 3,3'-Diaminobenzidine (DAB) and Wrap Red respectively. E-cadherin staining was used to define the epithelial compartment for better tissue segmentation. Hemotoxylin counterstain was used to define nuclei. The pTMA slide was scanned using Vectra platform. Epithelial cytoplasmic AGR2 staining intensity was analyzed using Nuance3.0.2 and inForm1.4.0 software. IBM SPSS Statistics 19 was used for data analysis.

Results: 362 total cores (94\%) were included for analysis. AGR2 expression was significantly elevated in prostatic adenocarcinomas compared to benign prostatic tissues. The highest expression was observed in clinically localized prostatic adenocarcinoma. Although there was a slight trend of higher AGR2 expression correlating with higher pathology stages and Gleason scores, the differences were not statistically significant. Compared to the clinically localized prostatic adenocarcinomas, AGR2 expression in the metastases, similar to that in high-grade intraepithelial neoplasia, was significantly lower $(\mathrm{p}<0.005)$.

Conclusions: AGR2 is overexpressed in prostatic adenocarcinomas and can be used as a potential tissue and blood based biomarker for detecting prostatic adenocarcinoma or recurrence. There are no significant associations between AGR2 expression and Gleason scores or pathological stages.

971 ERG Expression Is Associated with Increased Risk of Biochemical Relapse Following Radical Prostatectomy in Early Onset Prostate Cancer K-C Huang, SA Hegazy, M Dolph, B Donnelly, TA Bismar. University of Calgary and Calgary Laboratory Services, Calgary, AB, Canada; University of Calgary, Calgary, AB, Canada; Southern Alberta Cancer Institute and Tom Baker Cancer Center, Calgary, AB, Canada; The Prostate Cancer Center, Calgary, AB, Canada

Background: ERG expression has been identified in about $50 \%$ of clinically significant prostate cancer (PCA). Although ERG significance in PCA is debatable, it has been proposed to signify molecular subtype of PCA defined by specific progression pathways. The aim of this study is to assess the prognostic significance of ERG expression in cohort of early-onset PCA.

Design: ERG protein expression was investigated in a cohort of 121 men diagnosed at less than 50 years of age with localized PCA with an average follow-up time of 65.7 months. ERG expression was correlated with patients' clinical outcome and several clinical-pathological parameters.

Results: ERG expression was detected in 76/118 (64.4\%) analyzable patients' samples and showed interfocal heterogeneity (differences between foci) in $17 / 118(14.4 \%)$ patients. There was significant association between ERG expression and Gleason score $(p=0.022)$. However, ERG expression was not significantly associated with any other clinical-pathologic parameters, including pre-surgical PSA levels (mean and density), mean tumor volume, pathological stage, surgical margin status and lymph-vascular invasion. Investigating the prognostic significance of ERG, there was significant difference in the rate of biochemical relapse following radical prostatectomy, with ERG positive patients showing higher relapse rates compared to ERG negative patients $(\mathrm{p}=0.007)$. However, considering time till biochemical relapse post-radical prostatectomy, ERG expression showed positive insignificant trends with earlier biochemical relapse $(\mathrm{p}=0.071)$. Notably, and of great significant, in this cohort of early onset disease, none of the ERG negative PCA patients exhibited biochemical relapse. Conclusions: The study results suggests that ERG expression may be of added prognostic value in localized prostate cancer in patients with early onset PCA. However, the issue of interfocal heterogeneity observed in ERG expression may hamper its clinical utilization requiring evaluation of several tumor foci. Further larger studies are needed to confirm any prognostic significance of ERG expression in similar population.
972 Micropapillary Urothelial Carcinoma of the Bladder: Clinicopathologic Analysis of Patients with T1 Disease

H Huang, M Spaliviero, G Dalbagni, BH Bochner, JP Sfakianos, SM Donat, HW Herr, Y-B Chen, A Gopalan, SW Fine, SK Tickoo, VE Reuter, HA Al-Ahmadie. Memorial Sloan-Kettering Cancer Center, New York, NY; Montefiore Medical Center, Bronx, NY. Background: Controversies exist about the best management strategies for micropapillary carcinoma (MPC) of the bladder, with some advocating radical cystectomy even for low stage disease (pT1) citing rapid disease progression as a justification for this radical approach. Others have managed MPC similar to other highgrade urothelial carcinomas with aberrant differentiation. We report our experience of a well annotated cohort of MPC of the bladder, pathologically confirmed to be $\leq \mathrm{pT} 1$ disease.

Design: We identified 36 patients with MPC who underwent restaging transurethral resection of bladder tumor (TURBT) at our institution within 3 months of initial clinical stage T1 (cT1) cancer diagnosis. All H\&E slides were re-reviewed to confirm the diagnosis and to evaluate the amount of MPC component. Early cystectomy (within 3-month after restaging TURBT), or conservative management (intravesical Bacillus Calmette-Guérin, surveillance, or deferred cystectomy) was offered taking into account additional features at restaging (residual tumor volume, multifocality, presence of carcinoma in situ, lymphovascular invasion), surgical risk assessment, or patient's preference.

Results: Our cohort included 27 (75\%) men and 9 (25\%) women. Median age was 68 $(\mathrm{IQR} 63,77)$ years. At restaging, all patients had $\leq$ pT1 disease. Fifteen $(42 \%)$ patients underwent early cystectomy; 21 (58\%) were managed conservatively. Median follow-up time for cancer-specific mortality (CSM) and metastasis-free survival was 3.1 (IQR 1.1, 5.9 ) years and 3.1 (IQR 1.8,5.9) years, respectively. The 5-year cumulative incidence of CSM was $17 \%$ in the early cystectomy and $25 \%$ in the conservative management group $(\mathrm{p}=0.8)$. The 5 -year cumulative incidence of metastasis was $21 \%$ and $34 \%$, respectively $(\mathrm{p}=0.9)$. The extent of the MPC component was not significantly associated with CSM $(\mathrm{p}=0.5)$ or metastasis $(\mathrm{p}=0.9)$.

Conclusions: Using proper selection criteria, including clinical and pathologic factors, certain cT1 micropapillary urothelial carcinoma managed conservatively may have outcomes similar to those who have conventional urothelial carcinoma. The extent of the micropapillary component is not significantly associated with cancer-specific mortality or metastasis. The micropapillary component should be used in the context of other pathologic and clinical features to determine the proper management approach to these patients.

973 Evidence-Based Approach to Answering the Question: Is One Slide Enough to Make an Accurate Diagnosis of Prostate Carcinoma on Core Biopsy?

IF Hughes, K Hutchens, $H$ Chen, E Wojcik. Loyola University Medical Center, Maywood, IL.

Background: A new healthcare system is coming and in the changing environment it brings we must be prepared to provide for a greater population with the same or fewer resources. Many labs use multiple levels for the evaluation of prostate core biopsies, but are they necessary? While this kind of practice is widespread, it has no clear roots in evidence-based medicine. If prostate core biopsy specimens could be adequately evaluated using a single level while maintaining diagnostic accuracy, it would allow for more effective utilization of resources and shorter turn-around time. Decreased processing leads to lower costs both in terms of money and technician time. This study aims to provide an evidence-based approach to determining whether the diagnosis of prostatic adenocarcinoma by prostate core biopsy can accurately be made on a single slide.

Design: Slides from consecutive prostate core biopsy blocks from August 1, 2012 to August 7, 2013 with a diagnosis of adenocarcinoma were examined. Blocks were processed routinely to produce five levels. Hematoxylin and eosin-stained levels 1, 3, and 5 were each examined to determine if diagnostic material in agreement with the Gleason score and percent involvement of the final diagnosis was present.

Results: Out of 310 blocks with adenocarcinoma examined, five cases (2\%) displayed discrepancies in diagnosis between levels. In two of these cases the block was not properly faced before sectioning and tissue of interest was absent from the first level. Two other blocks were each part of a case in which other blocks showed carcinoma on all levels. In all five discordant cases, carcinoma was diagnosed as Gleason $3+4$ or lower involving $5 \%$ or less of the cores. Blocks with $\geq 10 \%$ carcinoma or Gleason score greater than $3+4$ showed no discrepancy.

\begin{tabular}{|c|c|c|c|c|c|c|c|}
\hline Case & $\begin{array}{l}\text { Final } \\
\text { Diagnosis } \\
\text { (Gleason, } \\
\%)\end{array}$ & Sections/Slide & \begin{tabular}{|l} 
Block \\
Faced?
\end{tabular} & $\begin{array}{l}\text { Other Blocks } \\
\text { in Case with } \\
\text { Carcinoma? }\end{array}$ & $\begin{array}{l}\text { PSA at } \\
\text { Biopsy }\end{array}$ & $\begin{array}{l}\text { Patient } \\
\text { Age }\end{array}$ & Treatment \\
\hline 1 & $3+3,5 \%$ & 2 & |Yes & Yes & 4.07 & | 70 & $\begin{array}{l}\text { Radical } \\
\text { Prostatectomy }\end{array}$ \\
\hline 2 & $3+4,<5 \%$ & 2 & No & No & 10.4 & 70 & \begin{tabular}{|l} 
Radical \\
Prostatectomy
\end{tabular} \\
\hline 3 & $3+3,<5 \%$ & 2 & Yes & Yes & 8.9 & 57 & Surveillance \\
\hline 4 & $3+3,5 \%$ & 2 & No & No & 5.59 & 59 & Surveillance \\
\hline 5 & $3+3,5 \%$ & 2 & Yes & No & 4.28 & 55 & Surveillance \\
\hline
\end{tabular}

Conclusions: If these blocks had been evaluated with a single level per block, one case with Gleason $3+3$ carcinoma in $5 \%$ of a core biopsy may have been missed. The patient opted for clinical followup with serial PSA tests only. Reduced processing would have saved $\$ 6,820$ for the examined cases alone and approximately $\$ 33,000$ for all prostate cores during this time ( $\$ 7$ per H\&E slide, $\$ 4$ per unstained slide). 


\section{Reduced Glucocorticoid Receptor Expression Predicts Bladder} Tumor Recurrence and Progression

H Ishiguro, T Kawahara, GJ Netto, H Miyamoto. Johns Hopkins Medical Institutions, Baltimore, MD; University of Rochester Medical Center, Rochester, NY.

Background: Preclinical evidence suggests that glucocorticoid-mediated glucocorticoid receptor (GR) signals play an inhibitory role in the growth of bladder cancer. However, the status of GR expression in bladder tumor and its prognostic significance remain largely unknown.

Design: We immunohistochemically stained for GR in 149 bladder tumor and matched 94 non-neoplastic bladder tissue specimens for which the expression levels of androgen receptor (AR), estrogen receptor (ER)- $\alpha$, and ER $\beta$ had been assessed. We then analyzed the association between GR expression and clinicopathologic features available for our patient cohort.

Results: GR was positive in 129 [87\%; 42 (28\%) weak, 51 (34\%) moderate, and 36 (24\%) strong] of 149 bladder tumors, which was significantly $(P=0.026)$ lower than in non-neoplastic urothelium [90 (96\%) of $94 ; 32(34 \%)$ weak, $33(35 \%)$ moderate, and $25(27 \%)$ strong]. There was no significant difference in the expression pattern between male versus female tumors. Fifty-one $(96 \%)$ of 53 lower grade tumors versus $78(81 \%)$ of 96 high-grade carcinomas $(P=0.011)$ and $81(96 \%)$ of 84 non-muscle-invasive (NMI) tumors versus $48(74 \%)$ of 65 muscle-invasive (MI) carcinomas $(P<0.001)$ were immunoreactive for GR. Kaplan-Meier and log-rank tests revealed that loss or weak positivity of GR significantly or marginally correlated with recurrence of NMI tumors $(P=0.025)$, progression of MI tumors $(P=0.082)$, and cancer-specific death in patients with MI tumor $(P=0.067)$. In male patients, the differences in survival rates were more significant [recurrence of NMI tumors $(P=0.019)$, progression of MI tumors $(P=0.029)$, and cancer-specific survival of MI tumors $(P=0.029)]$. Multivariate analysis further identified low GR expression as a strong predictor for recurrence of NMI tumors $(\mathrm{HR}=0.444,95 \% \mathrm{CI}=0.210-0.939, P=0.034)$. There were no significant correlation between GR versus AR $(P=0.230)$, a positive correlation between GR versus ER $\alpha$ $(P=0.004)$, and a negative correlation between GR versus ER $\beta(P=0.007)$.

Conclusions: GR expression was down-regulated in bladder tumors, compared with non-neoplastic bladders, and in high-grade/MI tumors, compared with lower grade/ NMI tumors. Decreased expression of GR predicted recurrence of NMI tumors, as an independent prognosticator, and progression of MI tumors, particularly in male patients. These results support experimental evidence indicating inhibitory effects of GR activation on bladder cancer progression and suggest potential interactions between GR and ER signals in bladder cancer cells.

975 Miniaturized Multiphoton Probe: A Potential "Virtual Histology" Tool for Targeted Biopsy of Prostate

M Jain, DM Huland, BD Robinson, MM Shevchuk, DG Ouzounov, JB Singh, P Singhal, $J$ Li, R Leung, CXu, AK Tewari. Weill Cornell Medical College, New York City, NY; Cornell University, Ithaca, NY; Weill Cornell Medical College, New York, NY.

Background: One of the major challenges in prostate cancer diagnosis and management is to reliably distinguish patients with indolent disease from those who have aggressive tumors. So far Gleason score is the best predictor of aggressiveness and mortality risk assessment. However, as the biopsy is done in a blinded manner and only samples $1 / 1000^{\text {th }}$ of the entire prostate gland, this leads to under- or over-estimation of the true cancer grade with concomitant (and potentially avoidable) side effects. Multiphoton microscopy (MPM) is an optical imaging technique capable of imaging tissue in realtime. Here we explore the potential of a miniaturized MPM probe-comparable in size to a biopsy needle-as a "virtual histology" tool for evaluation of the prostate.

Design: Benign and malignant areas were imaged with the miniaturized GRIN lensbased MPM probe in sections obtained from 13 fresh ex-vivo radical prostatectomy specimens. The MPM images were correlated with H\&E sections.

Results: In the benign areas, we could identify large glands with infolded epithelium and occasional concretions in the lumen. In the tumor areas, we recognized tumor morphologies, ranging from clusters of tiny glands with sharp rounded luminal borders (Figure 1) to areas with irregularly shaped/ poorly formed glands and nests of cells.

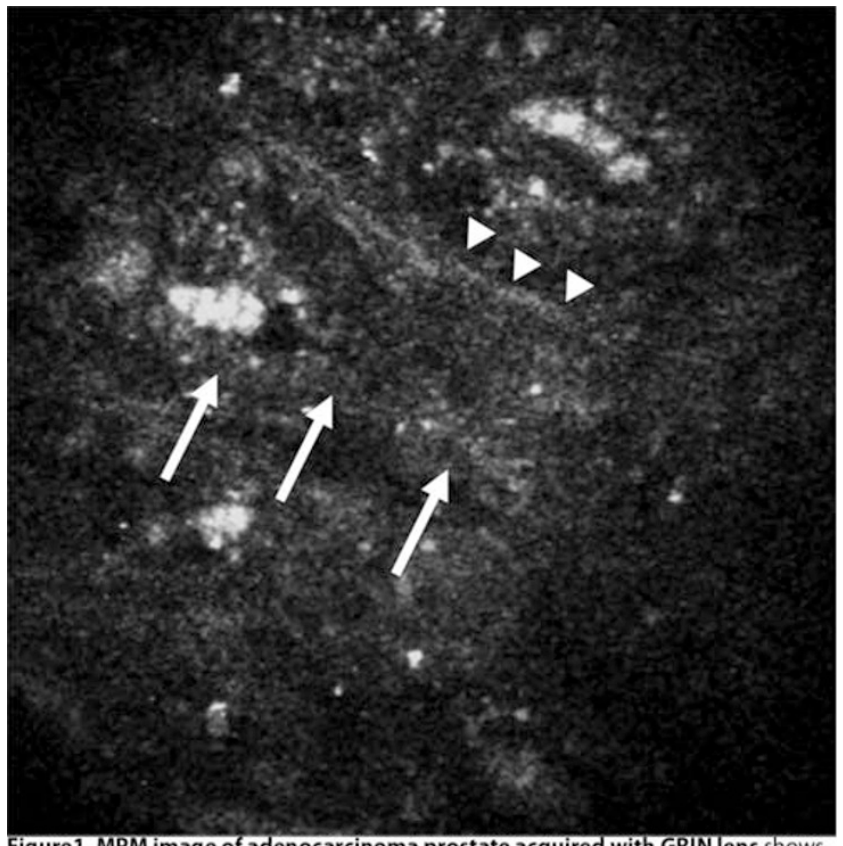

Figure 1. MPM image of adenocarcinoma prostate acquired with GRIN lens shows glands (cellular autofluorescence-color coded green; arrows) and stroma (second harmonic signals-color coded red; arrowheads). Total magnification $=1000 \mathrm{X}$.

The former pattern was confirmed to be low grade (Gleason6) tumor on final pathology, and the latter morphology corresponded to higher grade tumors (Gleason7+) on final histopathology.

Conclusions: GRIN lens-based MPM probe can be a valuable "virtual histology" tool for visualization of the suspicious areas of the prostate. This technology may not only decrease the sampling error by being able to visualize more areas of the prostate without extracting tissue, but also help target the most significant tumor. Both of these would allow for improved management of prostate cancer patients.

976 Tubulocystic Renal Cell Carcinoma Is an Entity Immunohistochemically and Genetically Distinct from Papillary Renal Cell Carcinoma

CL Jones, SR Williamson, JN Eble, DJ Grignon, $S$ Zhang, $M$ Wang, LA Baldrige, $L$ Wang, R Montironi, M Scarpelli, P-H Tan, E Comparat, L Cheng. Indiana University, Indianapolis, IN; Henry Ford Health System, Detroit, MI; Polytechnic University of the Marche Region and United Hospitals, Ancona, Italy; Singapore General Hospital, Singapore, Singapore; Groupe Hospitalier Pitié-Salpêtrière, Paris, France.

Background: Tubulocystic renal cell carcinoma (TCRCC) is a rare renal neoplasm that was originally considered as a low grade collecting duct carcinoma. Tumors are composed of cysts and tubules lined by cells with eosinophilic cytoplasm, often showing a hobnail-like configuration and prominent nucleoli. Some studies have suggested that TCRCC is related to papillary renal cell carcinoma based on similar cytogenetic and immunohistochemical profiles. We sought to compare and contrast molecular and immunohistochemical profiles of TCRCC to those of papillary renal cell carcinoma.

Design: Eight cases of tubulocystic renal cell carcinoma were identified. Clinical histories and hematoxylin and eosin slides of each case were reviewed to confirm the diagnosis. Formalin-fixed, paraffin embedded sections were subjected to a fluorescence in situ hybridization (FISH) assay containing probes for chromosomes 7 and 17. All cases were immunohistochemically stained with antibodies to AMACR (P504S), p63, cytokeratin 7, cytokeratin 20 and carbonic anhydrase IX.

Results: None of the 8 tumors exhibited gains of chromosomes 7 or 17 by FISH. Immunohistochemistry revealed all tumors to be nonreactive with antibodies to p63, cytokeratin 20 and AMACR. Only two tumors showed focal labeling with antibody to carbonic anhydrase IX $(25 \%)$ and only one showed a positive reaction with antibody to cytokeratin $7(12.5 \%)$.

Conclusions: The immunohistochemical profiles of the TCRCC included in this study are distinct from those of papillary renal cell carcinoma, particularly in the near uniform absence of reactivity with antibody to cytokeratin 7 ( 1 of 8 ) and AMACR ( 0 of 8). The absence of trisomies of chromosomes 7 or 17 provides additional support to the classification of TCRCC as a unique entity, distinct from papillary renal cell carcinoma.

977 Molecular Characterization of Basal Cell Carcinoma of the Prostate

CL Jones, GT MacLennan, R Montironi, SR Williamson, AO Osunkoya, $M$ Wang, $S$ Zhang, DJ Grignon, JN Eble, LA Baldrige, L Cheng. Indiana University, Indianapolis, IN; Case Western Reserve University, Cleveland, OH; Polytechnic University of the Marche Region and United Hospitals, Ancona, Italy; Henry Ford Health System, Detroit, MI; Emory University, Atlanta, GA.

Background: Basal cell carcinoma of the prostate is a rare tumor with a generally indolent clinical course. However, a small subset of these tumors behaves aggressively with local recurrence, extraprostatic extension and distant metastasis. This study was 
designed to investigate epidermal growth factor receptor $(E G F R)$ amplification and TMPRSS2-ERG gene rearrangement by fluorescence in situ hybridization (FISH). Additionally, protein expressions of EGFR, Her-2/neu, c-kit and androgen receptor were examined.

Design: Nine cases of basal cell carcinoma of the prostate were identified. Hematoxylin and eosin slides of each case were reviewed to confirm the diagnosis. Formalin-fixed, paraffin embedded sections were subjected to FISH with DNA probes for the EGFR gene and TMPRSS2-ERG rearrangement. All cases were additionally stained with antibodies to EGFR and Her-2/neu and were scored by evaluating the percentage of tumor cells staining positively and the intensity of staining $(0-3+$ scale $)$. Sections were also stained with antibodies to c-kit and androgen receptor.

Results: EGFR expression was present by immunohistochemistry in 6 of the $9(66 \%)$ cases, of which all strongly overexpressed EGFR (2+ to $3+$ ) in $40-90 \%$ of cells. FISH analysis revealed no EGFR gene amplification or TMPRSS2-ERG rearrangement in any tumor. Her-2/neu expression was not demonstrated in any of the cases. C-kit and androgen receptor expression were detected very focally and weakly in 4 cases (44\%) and 2 cases $(22 \%)$, respectively.

Conclusions: Our study shows that basal cell carcinomas of the prostate commonly express EGFR protein strongly; this phenomenon occurs in the absence of EGFR gene amplification. TMPRSS2-ERG rearrangement was not identified in the tumors we studied, confirming that the molecular pathogenesis differs from that of prostatic acinar adenocarcinoma. Additionally, Her-2/neu, c-kit and androgen receptor expression were either absent or showed only weak and focal reactivity. These findings suggest that anti-EGFR therapy may be potentially useful for the management of patients with aggressive basal cell carcinomas of the prostate that express EGFR.

\section{$978 \quad$ Nomogram for Predicting Resistance to Sunitinib in Patients} with Metastatic Clear Cell Renal Carcinoma

S-F Kammerer-Jacquet, M-A Belaud-Rotureau, E Oger, G Verhoest, A Lespagnol, J Edeline, S Jaillard, B Laguerre, E Vauleon, J Mosser, K Bensalah, N Rioux-Leclercq. CHU, Rennes, France; CRLCC, Rennes, France.

Background: Clear cell renal carcinoma (CCRC) is the most common histologic subtype of kidney cancer. $20 \%$ of CCRC patients present metastasis at diagnosis and $30 \%$ develop metastasis. Recent and better understanding of CRCC molecular pathways had permitted advances in its treatment with the development of antiangiogenic therapies. Among them, sunitinib, a multitargeted receptor tyrosine kinase inhibitor and particularly the receptor of vascular endothelial growth factor (VEGF) is the firstline therapy in metastatic CCRC (mCCRC). However, $30 \%$ of patients are refractory or acquire early resistance within first months of treatment. The aim of this work was to characterize mCCRC of patients resistant to sunitinib by comparing to mCCRC of long-term responders in order to find predictors of resistance.

Design: $14 \mathrm{mCCRC}$ patients treated by sunitinib were retrospectively included to define a group of 7 resistant patients and a group of 7 long-term responders according to response evaluation criteria in solid tumors and progression-free survival. 14 corresponding formalin fixed paraffin embedded CCRC were analysed morphologically and by immunohistochemistry (IHC). Array comparative genomic hybridization (CGH-array) and complete VHL gene status were achieved on 14 corresponding frozen CCRC. Univariate analysis used exact Fisher test, bilateral $\mathrm{p}$ values $<0,05$ were considered significant. Variables with a $\mathrm{p} \leq 0,10$ in the univariate analysis were selected for multivariate analysis using a logistic regression model.

Results: Intratumoral overexpression ( $>60 \%$, IHC) of VEGF and chemokine receptor CXCR4 were significantly associated with resistance to sunitinib ( $\mathrm{p}=0,03$ and $\mathrm{p}<0,01$ respectively). The addition of complete loss of $14 \mathrm{q}$ (CGH-array) associated with resistance $(\mathrm{p}=0,10)$ built a nomogram predicting resistance to sunitinib in $\mathrm{mCCRC}$ patients. If two or more variables were present, all patients were resistant. On the contrary, if one or less variable was present, all patients were long-term responders to sunitinib.

Conclusions: For the first time, a nomogram stratifying mCCRC patients according to VEGFA and CXCR4 high expression and loss of 14q is proposed. Given the lack of biomarkers to predict response to sunitinib, this nomogram, if validated by a larger prospective study, will offer opportunity to greatly improve strategy in mCCRC treatment.

979 A Comparative Immunohistochemical Study of Gonadoblastomas (GB), Sertoli Cell Nodules with Intratubular Germ Cell Neoplasia (SCN with IGCNU) and Hamartomas of the Androgen Insensitivity Syndrome (AIS)

C-S Kao, TM Ulbright, MT Idrees. Indiana University, Indianapolis, IN.

Background: GBs and hamartomas of AIS are distinctive lesions of disorders of sex development (intersex syndromes). Some have interpreted SCNs with IGCNU in the testes lacking dysgenetic features as GBs. FOXL2 is essential for ovarian differentiation whereas SOX9 is required for testicular organogenesis. SF1, in addition to regulating steroidogenesis in the gonads and adrenal gland, up-regulates SOX9 transcription. Prior studies demonstrated that the sex cord-stromal cells of GB are positive for FOLX2 and negative for SOX9. The expression of SOX9 and FOXL2 in SCNs with IGCNU and hamartomas of AIS have not been studied. We investigated the SOX9, FOXL2, and SF 1 reactivity in these entities.

Design: We identified $10 \mathrm{GBs}$ (all phenotypic females with karyotyping showing Y chromosomes), $11 \mathrm{SCNs}$ with IGCNU in males with germ cell tumors, and 8 hamartomas in AIS patients. Immunohistochemical stains were performed. Positivity was defined as $>10 \%$ of the sex cord tumor cells showing at least moderately intense reactivity. Results: The results (\# of positive cases/\# of cases stained) are listed in the table. GBs showed $100 \%$ reactivity with FOXL2, in addition to frequent SOX9 (80\%) and SF1
$(90 \%)$ expression. The sex cord-stromal cells in SCNs with IGCNU were uniformly positive for SOX9 and SF1 while negative for FOXL2; this identical pattern was also found in the hamartomas in AIS.

\begin{tabular}{|l|l|l|l||}
\hline Lesion & SOX9 & FOXL2 & SF1 \\
\hline Gonadoblastoma & $8 / 10$ & $10 / 10$ & $9 / 10$ \\
\hline SCN with IGCNU & $11 / 11$ & $0 / 10$ & $8 / 8$ \\
\hline Hamartoma in AIS & $8 / 8$ & $0 / 8$ & $8 / 8$ \\
\hline
\end{tabular}

Conclusions: Our study demonstrates coexpression of SOX9 and FOXL2 in the sex cord-stromal cells of indeterminate differentiation in GBs. This may be evidence that these morphologically undifferentiated sex cord cells show divergent differentiation and is in accordance with older studies demonstrating Charcot-BÖttcher filaments (a Sertoli cell specific structure) in these cells on ultrastructural examination as well as recent studies showing FOXL2 expression. The absence of FOXL2 reactivity in the SCNs with IGNCU serves to distinguish them from true GBs. The Sertoli cell nature of the immature sex cord cells in the hamartomas of AIS is supported by their SOX9 positivity and FOXL2 negativity.

980 The Utility of Immunostaining for NUT, GAGE7, and NY-ESO-1 in the Diagnosis of Spermatocytic Seminoma

C-S Kao, S Badve, TM Ulbright. Indiana University School of Medicine, Indianapolis, IN.

Background: Spermatocytic seminoma (SS) may be misinterpreted as other types of germ cell tumor. The distinction is important since the treatments and prognoses are different. In cases with atypical presentations or lacking classic morphology, immunostains may be reassuring; however, few are reactive for SS. A monoclonal antibody directed against nuclear protein in testis (NUT) was recently developed and shown to be positive in some seminomas/dysgerminomas, but its reactivity for SSs has not been studied. Cancer/testis antigens have been shown to be expressed in SSs, but a comprehensive study including a greater number of cases and other germ cell tumors is lacking. Our goal was to evaluate the reactivity of NUT, GAGE7, and NY-ESO-1 antibodies in SSs compared to that in its potential mimics.

Design: 18 SSs (2 with sarcomatous transformation), 28 usual seminomas, 26 embryonal carcinomas, and 21 solid yolk sac tumors (7 hepatoid and 14 non-hepatoid) were selected. Immunostains on whole sections of formalin-fixed, paraffin-embedded tumors were conducted against NUT, GAGE7, and NY-ESO-1. Only nuclear reactivity was considered positive. The results were scored by adding extent $(E)$ and intensity (I) of the staining reactions. Extent of $<1 \%=0, \geq 1 \%$ but $<10 \%=1, \geq 10 \%$ but $<50 \%$ $=2$, and $\geq 50 \%=3$. Intensity was compared to the positive internal controls of normal spermatogenic cells: same $=2$, less $=1$, and negative/equivocal $=0$. Positive reactivity was defined as $E+I \geq 4$. All negative cases without working internal controls were deemed non-informative and excluded from data tabulation.

Results: The results of tumor positivity are summarized in the table. The sensitivity and specificity of the three antibodies, respectively, for SS using the $\mathrm{E}+\mathrm{I} \geq 4$ cutoff were as follows: NUT- $69 \%$ and $92 \%$, GAGE7- $64 \%$ and $99 \%$, and NY-ESO- $1-81 \%$ and $94 \%$. In the sarcoma component of 2 SSs no reactivity occurred for any of the three antigens. Positive Reactivity of Testicular Germ Cell Tumors for NUT, GAGE7, and NY-ESO-1

\begin{tabular}{|l|l|l|l||}
\hline Diagnosis & NUT & GAGE7 & NY-ESO-1 \\
\hline SS & $69 \%$ & $64 \%$ & $81 \%$ \\
\hline Seminoma & $19 \%$ & $4 \%$ & $13 \%$ \\
\hline EC & $0 \%$ & $0 \%$ & $0 \%$ \\
\hline Solid YST, hepatoid & $14 \%$ & $0 \%$ & $14 \%$ \\
\hline Solid YST, non-hepatoid & $0 \%$ & $0 \%$ & $0 \%$ \\
\hline
\end{tabular}

$\mathrm{SS}=$ spermatocytic seminoma; $\mathrm{EC}=$ embryonal carcinoma; $\mathrm{YST}=$ yolk sac tumor

Conclusions: We conclude that all three stains are variably positive in SS and have good specificity for SS when there is strong and at least multifocal nuclear reactivity. Although seminomas and hepatoid solid yolk sac tumors may show reactivity, it is usually limited and weak. Embryonal carcinomas and non-hepatoid solid yolk sac tumors are consistently negative.

981 "Somatic-Type" Malignancies Arising from Testicular Germ Cell Tumors (TGCTs): A Clinicopathologic Study with Emphasis on Glandular Tumors Supporting Frequent Yolk Sac Tumor (YST) Origin

C-S Kao, MJ Magers, KR Rice, TM Ulbright. Indiana University, Indianapolis, IN. Background: Somatic-type malignancies (SM) in patients with TGCTs are rare. We investigated their morphologic spectrum, immunohistochemical (IHC) profiles, survival outcomes and relationship to YST.

Design: We identified 122 cases of SM of TGCT origin from 107 patients from 19852012. PNET was excluded due to distinct management. The H\&E slides were reviewed, IHC performed and followup information obtained.

Results: Patients were 15-68 years old (median, 30). Tumors ranged from $0.7-30 \mathrm{~cm}$ (mean, 7.6$)$ and involved retroperitoneum (65\%), abdomen/pelvis (10\%), lung (8\%), mediastinum (4\%), and testis $(4 \%)$. Most initial diagnoses were sarcomas $(n=66)$ or carcinomas $(\mathrm{CA})(\mathrm{n}=50)$. On review and IHC study (Table 1), 7 were reclassified as glandular YSTs (GYST) based on GPC3 and/or AFP positivity and scant/absent reactivity for EMA \& CK7. GYSTs frequently (57\%) had sub and/or supranuclear vacuoles (endometrioid-like) whereas adenoCAs were more frequently intestinal $(18 \%)$ or mucinous (18\%) than endometrioid-like (9\%). GYSTs expressed CDX2 more frequently $(83 \%)$ than adenoCAs $(63 \%)$ while the latter more frequently expressed MUC proteins 2, 4, 5 \& 6 (6-36\% compared to $0-20 \%)$. Both were often positive for SALL4, BerEp4 \& MOC31, and all negative for TTF1. Followup (range 1-300 mo; mean, 76) showed $50 \%$ and $33 \%$ with GYST and adenoCA died of disease, respectively. 27 spindle cell cases were reclassified as sarcomatoid YSTs (SYST) based on AE1/3 and GPC3 reactivity. SYSTs were often (56\%) SALL4 positive while sarcomas were 
all negative. Followup (range 1-327 mo; mean, 56) showed 50\% and 28\% with SYST and sarcoma died of disease, respectively; most were high grade.

Table 1. Glandular SM

\begin{tabular}{|l||l||l||l||l||l||l|}
\hline Tumor (\# of cases) & SALL4 & GPC3 & AFP & CK7 & EMA & CDX2 \\
\hline
\end{tabular}

Tumor (\# of cases)

\begin{tabular}{|l|l|l|l|l|l|l|l|l|l|l|l|l|l|l|}
\hline $5 / 7$ & $6 / 7$ & $5 / 7$ & $1 / 7$ & $5 / 7$ & $5 / 6$ \\
\hline
\end{tabular}

\begin{tabular}{|l||l|l|l|l|l|l|l|l|}
\hline Indeterminate (GYST or AdenoCA) (3)* & $2 / 3$ & $1 / 3$ & $1 / 3$ & $0 / 3$ & $1 / 3$ & $2 / 2$ \\
\hline \hline
\end{tabular}

AdenoCA(2 NOS, 6 madinous, 3 achar cen

type, 3 papillary/villoglandular, 1 signet-ring cell, 1 clear cell)

\begin{tabular}{|l|l|l|l|l|l}
$10 / 28$ & $2 / 28$ & $1 / 28$ & $12 / 28$ & $22 / 27$ & $17 / 27$ \\
\hline
\end{tabular}

*Originally diagnosed as adenoCA

Conclusions: SMs of TGCT origin show overlapping morphology, IHC features, and aggressive behavior with special types of YST. Many apparent SMs are unusual, chemoresistant forms of YST. The indeterminate glandular tumors may represent those in transition between GYST and adenocarcinoma. Tumor grade is prognostically important in spindle cell tumors, and approximately one-third are SYSTs. SALL4 is useful in recognizing glandular tumors and SYSTs of TGCT origin but not sarcomas.

982 Profilin-1 Expression Is Associated with High Grade and Stage and Decreased Progression-Free Survival in Renal Cell Carcinoma

JR Karamchandani, NMA White, A Scorilas, MY Gabril, E Filter, GM Yousef. University of Toronto, Toronto, ON, Canada; Mount Sinai Hospital, Toronto, ON, Canada; University of Athens, Athens, Greece; London Health Sciences, London, ON, Canada. Background: Renal cell carcinoma(RCC) is the most common kidney tumor in adults and is associated with significant morbidity and mortality world-wide. Profilin-1(Pfn1) particpates in cytoskeletal remodling by regulating actin polymerizaiton. $\mathrm{Pfn} 1$ has been identified as over-expressed in RCC tissues and cell lines. Recent proteomic studies have also identified compatively increased Pfn 1 expression in cases of RCC with metastatic behavior. We hypothesized that increased Pfn1 expression would be associated with higher tumor grade(GR) and stage(STG).

Design: We built a tissue microarray(TMA) containing duplicate cores from 346 cases of primary RCC. We assesed the tumor GR and STG from chart review, as well any record of metastasis(indicating progression / end of disease-free survival(DFS)). 5- $\mu \mathrm{m}$ sections were stained for Pfn1 by immunohistochemistry. Immunoexpression of Pfn1 was evaluated by a pathologist and semi-quantitatively classified as absent, moderate, or strong and assessed for both cytoplasmic(CYT) and nuclear(NUC) expression. Differences beween low(1-2) and high(3-4) GR and STG tumors was calculated by either Fisher's exact test or Pearson Chi-square analysis.

Results: Both strong CYT and NUC expression of Pfn1 were associated with higher tumor grade and stage.

Table 1: CYT Pfn1 vs GR and STG

\begin{tabular}{|c|c|c|c|c|}
\hline & $\mathrm{N}$ & Moderate $(\%)$ & Strong $(\%)$ & p-val \\
\hline \multicolumn{5}{|l|}{ GR } \\
\hline $1-2$ & 206 & $30(14.6)$ & $176(85.4)$ & \\
\hline $3-4$ & 140 & $4(2.8)$ & $136(97.1)$ & $.001^{*}$ \\
\hline \multicolumn{5}{|l|}{ STG } \\
\hline $1-2$ & 211 & $27(12.8)$ & $184(87.2)$ & \\
\hline $3-4$ & 91 & $4(4.4)$ & $87(95.6)$ & $0.018^{*}$ \\
\hline
\end{tabular}

$* \mathrm{p}<.05$ indicates statistical significance

Table 2: NUC Pfn1 vs GR and STC

\begin{tabular}{|c|c|c|c|c|c|}
\hline & $\mathrm{N}$ & Absent $(\%)$ & Moderate $(\%)$ & Strong $(\%)$ & p-val \\
\hline \multicolumn{6}{|l|}{ GR } \\
\hline $1-2$ & 214 & $8(3.7)$ & $82(38.3)$ & $124(57.9)$ & \\
\hline $3-4$ & 140 & $7(5)$ & $63(45)$ & $70(50)$ & $0.043^{*}$ \\
\hline \multicolumn{6}{|l|}{ STG } \\
\hline $1-2$ & 219 & $11(5)$ & $81(37)$ & $127(58)$ & \\
\hline $3-4$ & 93 & $3(3.2)$ & $50(53.8)$ & $40(43)$ & $0.023^{*}$ \\
\hline
\end{tabular}

$* \mathrm{p}<.05$ indicates statistical significance

High CYT Pfn1 expression was also associated with decreased DFS.

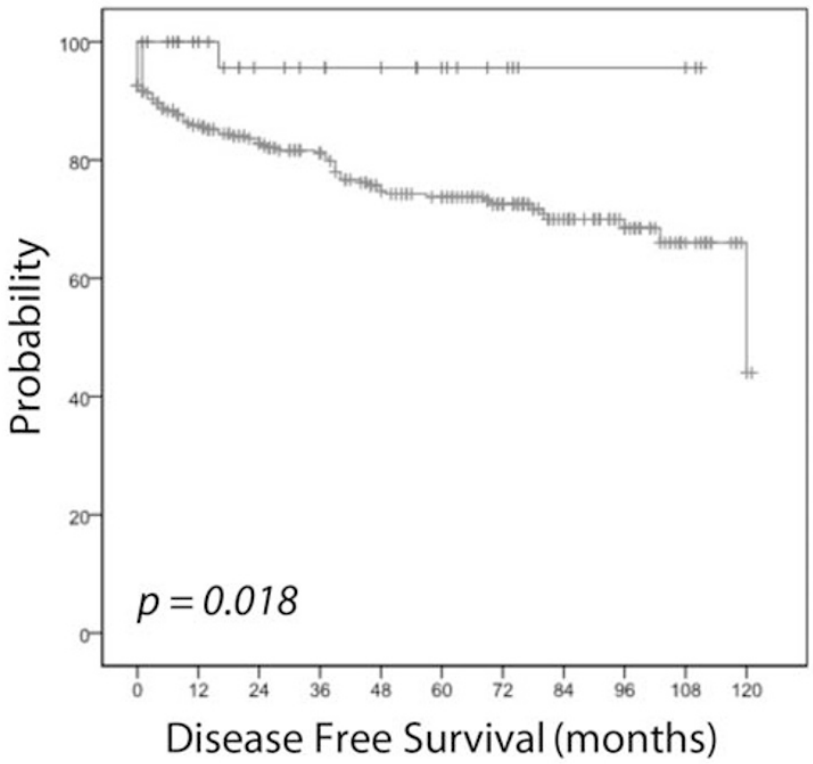

Conclusions: Pfn1 is a useful biomarker in RCC that shows increased cytoplasmic and nuclear expression in tumors of higher grade and stage. Pfn 1 expression is also associated with faster disease progression.

$983 \quad$ NFATc1 Expression Is Elevated in Prostate Cancer and Is an Independent Predictor of Biochemical Recurrence after Radical Prostatectomy

T Kawahara, H Ishiguro, Y Li, H Miyamoto. Johns Hopkins Medical Institutions, Baltimore, MD; University of Rochester Medical Center, Rochester, NY.

Background: Recent studies have suggested that some isoforms of nuclear factor of activated T-cells (NFAT), originally identified in immune cells as a transcription factor, promote cell survival, invasion, and angiogenesis. However, the status of NFAT expression in prostate cancer and its prognostic significance remain uncertain

Design: We immunohistochemically stained for NFATc1 in 225 radical prostatectomy specimens. We then evaluated the association between NFATc1 expression and clinicopathologic profile available for our patient cohort.

Results: Positive signals were detected predominantly in cytoplasm of benign and malignant epithelial cells. NFATc1 was positive in $91[40 \% ; 80(36 \%)$ weak and $11(5 \%)$ moderate] of 225 benign, 172 [82\%; 102 (48\%) weak, 67 (32\%) moderate, and $3(1 \%)$ strong] of 211 high-grade prostatic intraepithelial neoplasia (PIN), and 196 [87\%; 54 (24\%) weak, $114(51 \%)$ moderate, and $28(12 \%)$ strong] of 225 carcinoma tissues. Thus, the levels of NFATc1 expression were significantly higher in carcinoma (positivity and score, $P<0.001$ ) or PIN (positivity and score, $P<0.001$ ) than in benign and in carcinoma than in PIN (positivity, $P=0.115$; score, $P<0.001$ ). NFATc1 score was also higher in Gleason score $>7$ tumors than in Gleason score $\leq 6$ tumors $(P=0.012)$. There were no statistically significant correlations between NFATc1 stain and other clinicopathologic profile, such as pathologic stage $(\mathrm{pT} / \mathrm{pN})$ or preoperative prostate-specific antigen (PSA) level. Kaplan-Meier and log-rank tests revealed that patients with NFATc1-positive cancer $(P=0.057)$ or moderately/strongly NFATc1-positive cancer $(P=0.004)$ had a higher risk for biochemical/PSA recurrence. Multivariate Cox model further identified NFATc1 positivity (hazard ratio $=2.000 ; 95 \%$ confidence interval $=1.085-3.686 ; P=$ 0.026 ) or high NFATc1 expression (hazard ratio $=6.120 ; 95 \%$ confidence interval $=$ $1.367-27.404 ; P=0.018$ ) as a strong predictor for recurrence.

Conclusions: NFATc1 expression was up-regulated in PIN and prostate cancer, compared with non-neoplastic prostatic epithelium, and in cancer, compared with PIN. These results imply the involvement of NFATc1 in prostate cancer development and progression. Moreover, NFATc1 expression could be a reliable prognosticator in men who undergo radical prostatectomy.

984 Metastatic Prostate Carcinoma in Pelvic Lymph Nodes Following Neoadjuvant Chemotherapy: The Eyes Have It!

EL Kehr, P Masry, R Lis, M Loda, MS Hirsch. Brigham and Women's Hospital, Boston, MA.

Background: The use of neoadjuvant (NA) chemotherapy prior to radical prostatectomy (RP) is being considered for a subset of patients with high risk prostatic adenocarcinoma. Morphology after NA therapy includes inconspicuous cytology and single cells characterized by pale cytoplasm and small nuclei. Such treated tumor cells can be difficult to identify in lymph nodes (LNs), raising concern for under staging patients. The purpose of this study was to evaluate the role of immunohistochemistry (IHC) in identifying LN metastases in the setting of NA treated prostate cancer.

Design: A retrospective review identified 30 patients who were treated with NA chemotherapy (Abiaterone $n=22$; MDV3100 $n=8$ ) prior to RP. All available pelvic LNs blocks were stained with AE1/AE3, prostate specific antigen (PrSA), prostate specific acid phosphatase (PrAP), and androgen receptor (AR). The IHC slides were scored 'blind' and then retrospectively compared to H\&E slides and pathology reports. Clinical records were reviewed for demographical data, clinical follow-up including non-LN metastases, and mortality.

Results: The median age was 57 (range 47-71), and all patients were enrolled in a clinical trial. In total, $151 \mathrm{LN}$ blocks from 30 patients were immunostained. Metastatic carcinoma was found by IHC in 4 LNs from 2 patients. All 4 metastases were positive for AR, 3 of 4 were positive for AE1/AE3, 2 of 4 were positive for PrSA, and PrAP was negative in all metastases. All 4 LNs metastases were identified by H\&E at the time of RP. All remaining LNs were negative for the 4 biomarkers. The median time since RP was 26.4 months. Distant non-LN metastases were subsequently found in 2 men, 1 of whom had a positive LN at RP. Isolated biochemical recurrence with unknown site of recurrence has been found in 2 additional patients, who had negative LNs at RP. One patient died of disease and his LNs were negative.

Conclusions: Prostatic adenocarcinoma treated with NA chemotherapy is often associated with inconspicuous morphologic features which can be difficult to identify in resected LNs. Despite this, all LN metastases in this series were identified by H\&E, and no additional LN metastases were found by IHC. These findings suggest that routine use of IHC on LNs from NA treated prostate carcinomas is not necessary. Nevertheless, for suspicious small foci of atypical cells in a LN, AR and AE1/AE3 have greater sensitivity over PrSA and PrAP for confirming a metastatic deposit. Additionally, the absence of $\mathrm{LN}$ metastasis at the time of RP can still be associated with poor clinical outcome.

985 Polyomavirus in Renal Allografts

DJ Kenan, HH Singh, V Nickeleit. University of North Carolina, Chapel Hill, NC.

Background: BK polyomavirus (BKV) is nearly ubiquitous in human populations, establishing latency after primary infection. BKV is associated with polyomavirus allograft nephropathy in $2-4 \%$ of kidney transplant recipients. Rarely, BKV has 
been associated with aggressive forms of urothelial carcinoma in the setting of renal transplantation. Although increasingly recognized, the mechanistic association between BKV and allograft tumors is not understood.

Design: Two transplant nephrectomies with high grade invasive transitional cell and collecting duct-like carcinoma were analyzed. Laser capture microdissection on formalin fixed normal and tumor tissue was performed and DNA extracted. Tissue sections were analyzed by immunohistochemistry (targeting SV-40 T and VP capsid antigens), in-situ hybridization and electron microscopy. Molecular studies were based on quantitative PCR assays and targeted genomic DNA sequencing. Western blot analysis was employed to further assess viral production.

Results: Both study tumors strongly expressed T-antigen (a BKV proliferation marker) in tumor cell nuclei but not in adjacent normal tubules/parenchyma or urothelium. No signals were seen for VP capsid antigens (a marker for mature virions), by in-situ hybridization, western blotting, or electron microscopy in any tissue compartment. A TaqMan PCR assay showed BKV DNA sequences (encoding T and VP) in relatively low copy numbers in the laser dissected tumor components but not in the adjacent normal parenchyma. No PCR amplification products were detected with primers specific for JC-polyomavirus. Conventional PCR further demonstrated the presence of the full length BKV genome. Sequencing of the PCR fragments enabled determination of the BKV genotypes.

Conclusions: These new findings demonstrate that BK-polyomavirus is closely associated with high grade renal and urothelial carcinomas occurring in kidney transplant recipients. The presence of BKV is restricted to tumor cells, and it is not associated with BKV replication or a productive infection. A number of mechanisms can account for possible oncogenesis by BKV, and these are being further evaluated.

\section{PTEN Loss in Upper Tract Urothelial Carcinoma Is Associated with Biologically Aggressive Disease}

F Khani, DJ Lee, E Xylinas, MS Rieken, AM De Marzo, DS Scherr, SF Shariat, BD Robinson. Weill Cornell Medical College, New York, NY; University of Vienna, Vienna, Austria.

Background: Several previous studies have shown that PTEN loss in urothelial carcinomas of the bladder (UCB) is associated with a more aggressive phenotype and worse prognosis. While most tumors of the upper urinary tract-like most tumors of the bladder-are urothelial carcinomas, clinical, pathologic, and molecular differences do exist between upper tract urothelial carcinoma (UTUC) and UCB. To our knowledge, only one previous study, which included 99 patients, has evaluated PTEN loss in UTUC, and in that cohort the authors demonstrated an association of PTEN loss with worse outcomes, although the finding did not achieve statistical significance. The aim of our study was to evaluate PTEN loss in a larger cohort of UTUC patients and its association with clinical outcomes.

Design: Immunohistochemistry for PTEN was performed on TMAs constructed from 753 consecutive patients treated with radical nephroureterectomy between 1991 and 2008. Uni- and multivariable Cox-regression analyses evaluated the association of PTEN loss with disease recurrence, cancer-specific mortality, and all-cause mortality. Results: PTEN loss was noted in 45 (7.4\%) of the 753 cases. Loss of PTEN correlated with higher tumor stage, higher tumor grade, lymphovascular invasion, and the presence of nodal metastasis ( $<<0.05$ for all). With a median follow-up of 27 months (IQR 12-53), 191 patients (25\%) experienced disease recurrence, and $165(22 \%)$ died of the disease. Patients whose tumors showed PTEN loss had significantly worse recurrence-free survival ( $39 \%$ vs. $70 \%$, p $<0.01$ ), cancer-specific survival ( $44 \%$ vs. $70 \%$, $\mathrm{p}<0.01)$, and overall survival $(23 \%$ vs. $61 \%, \mathrm{p}<0.01)$ at five years compared to patients whose tumors retained PTEN expression. However, in multivariable Cox regression analyses, which adjusted for the effects of standard clinicopathologic features, PTEN loss was not an independent predictor of disease recurrence, cancer-specific mortality, or all-cause mortality.

Conclusions: Compared to previous studies evaluating PTEN in UCB, PTEN loss appears to be less frequent in UTUC. While PTEN loss was not an independent predictor of survival in our cohort, it was associated with higher tumor stage, grade, and the presence of lymph node metastasis. Thus, evaluation of tumors for PTEN loss in the pre-operative setting may help clinicians identify patients with potentially more aggressive disease.

987 Insulin-Like Growth Factor mRNA-Binding Protein 3 (IMP3) Expression Helps Prognostication in Patients with Upper Tract Urothelial Carcinoma

F Khani, DJ Lee, E Xylinas, MS Rieken, DS Scherr, SF Shariat, BD Robinson. Weill Cornell Medical College, New York, NY; Medical University of Vienna, Vienna, Austria. Background: Upper tract urothelial carcinoma (UTUC) is a clinically heterogeneous disease that lacks high quality trials that provide definitive prognostic criteria. Insulinlike growth factor mRNA binding protein 3 (IMP3) has been associated with outcomes in urothelial carcinoma of the bladder, but it has not been studied yet in UTUC. In the present study, we sought to evaluate the association of the oncofetal protein IMP3 with oncologic outcomes in patients with UTUC treated with radical nephroureterectomy (RNU).

Design: We investigated the expression of IMP3 by immunohistochemistry and its association with clinical outcomes using tissue microarrays constructed from 753 consecutive patients treated with RNU at 7 international institutions between 1991 and 2008. Uni- and multivariable Cox-regression analyses evaluated the association of IMP3 protein expression with disease recurrence, cancer-specific mortality, and all-cause mortality.

Results: IMP3 was expressed in $12.2 \%$ of UTUC ( $\mathrm{N}=76$ ). Expression was tumor-specific and correlated with higher stages/grades. Within a median follow-up of 27 months (IQR $12-53), 191$ patients $(25.4 \%)$ experienced disease recurrence and $165(21.9 \%)$ died of the disease. Patients whose tumors showed positive IMP3 expression had significantly worse recurrence-free survival $(27.4 \%$ vs. $75.1 \%, p<0.01)$, cancer-specific survival $(34.5 \%$ vs. $78.9 \%, \mathrm{p}<0.01)$, and overall survival $(15.6 \%$ vs. $64.8 \%, \mathrm{p}<0.01)$ at five years compared to patients with IMP3-negative tumors. In multivariable Cox regression analyses, which adjusted for the effects of standard clinicopathologic features, IMP3 expression was independently associated with disease recurrence (HR $1.85, \mathrm{p}<0.01$ ), cancer-specific mortality (HR 2.11, $\mathrm{p}<0.01$ ), and all-cause mortality (HR 2.09, $\mathrm{p}<0.01$ ). Conclusions: IMP3 expression was independently associated with disease recurrence, cancer-specific mortality, and all-cause mortality. IMP3 expression may help riskstratify patients who would benefit from adjuvant therapy or closer surveillance after RNU. Further studies are needed to evaluate the clinical value of IMP3 as a prognostic biomarker and potential target for therapy in UTUC.

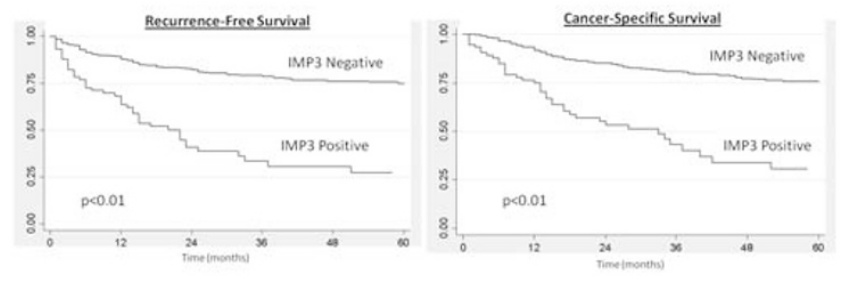

988 The Aberrant Expression of Common Genitourinary Immunohistochemical Stains in Diffuse Large B Cell Lymphoma

P Khattar, J Sarungbam, HK Islam, F Shakil, M Zhong. Westchester Medical Center, Valhalla, NY.

Background: Diffuse large B-cell lymphoma (DLBCL) is the most common type of non-Hodgkin lymphoma. It can arise in virtually any part of the body and may mimic carcinomas, morphologically. In addition to histopathological features, the knowledge of immunohistochemical (IHC) stain pitfall in diffuse large B cell lymphoma is also critical for pathologist to avoid misdiagnosis. The expression of common genitourinary (GU) IHC stains has not been systemically studied yet. Hence, in this study, we evaluated the expression of common GU IHC stains in DLBCL.

Design: A tissue microarray (TMA) with 46 cases of DLBCL was constructed. These included 13/46 (28\%) germinal centre B-cell-like (GBC) type, 16/46 cases (35\%) activated B-cell-like (ABC) type, and 17/46 cases (37\%) unclassified. The TMA slides were stained with the following antibodies: PAX8, p63, p40, GATA3, Desmoglein 3, PAX5 and AMACR. The expression and intensity of stains were evaluated and compared with subgroups of DLBCL origin.

Results: P63, common urothelial marker showed positivity in $46 \%, 31 \%$ and $59 \%$ of $\mathrm{GCB}, \mathrm{ABC}$ and unclassified type of DLBCL, respectively. PAX8 and PAX5 showed strong diffuse positivity in all 46 cases DLBCL. P40, GATA3, AMACR and Desmoglein3 were completely negative in all the cases.

Conclusions: 1 . P40 is more specific marker than p63 for differentiating urothelial carcinoma vs. DLBCL

2. GATA3, a new urothelial marker, was found to be negative in DLBCL but the background $\mathrm{T}$ cell lymphocytes showed strong positivity.

3. PAX8 has high expression in DLBCL due to cross- reaction with PAX5.

4. There was no significant difference among different DLBCL subtype in terms of Genitourinary IHC stains.

$989 \quad$ Examination of a Recently Proposed Grading System for Clear Cell Renal Cell Carcinoma (CCRCC)

LY Khor, JP Reynolds, CG Przybycin. Cleveland Clinic, Cleveland, $\mathrm{OH}$.

Background: The Fuhrman grade is the most widely used system for grading CCRCC. A novel grading system based on nucleolar prominence was recently applied to a large series of RCC with incorporation of tumor necrosis, lending additional predictive power. We attempted to validate this newly described grading approach.

Design: We reviewed 215 consecutive CCRCC specimens obtained from 1985-1997. Grade was assigned as follows: grade 1: absent or inconspicuous nucleoli at 40X magnification; grade 2: nucleoli conspicuous at $40 \mathrm{X}$ but inconspicuous or invisible at 10X; grade 3: nucleoli conspicuous at 10X; grade 4: extreme nuclear pleomorphism and/or rhabdoid or sarcomatoid component. Presence or absence of necrosis was also noted, and stage was confirmed. Follow-up information was obtained.

Results: Of the 215 cases, 116 were stage pT1, 25 pT2, and 74 pT3. Tumor size ranged from 0.6 to $17 \mathrm{~cm}$ (mean $5.7 \mathrm{~cm}$ ). Tumors were divided into groups based on nucleolar grade and presence of necrosis and correlated with follow-up status, as shown in the table below.

Survival by Grade Groups

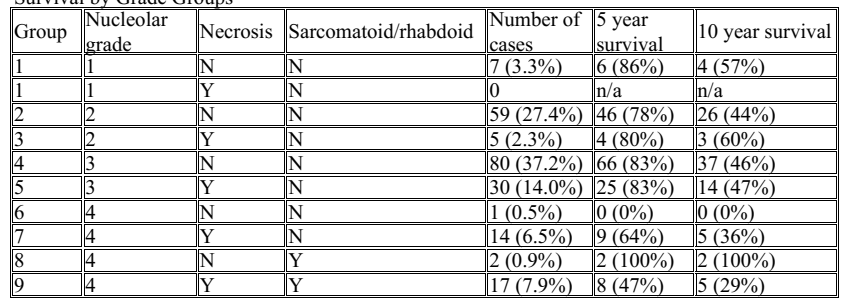

Conclusions: We were not able to validate the findings of the previous study by incorporating tumor necrosis into grade using a smaller set of cases. 
990 Aggressive Prostatic Adenocarcinoma Cancer (PAC) in African American Males (AAM): Molecular Characterization by miRNA Expression Profiling May Explain Aggressive Behavior

$R$ Kolhe, P Vasa, A Rahardja, S Kavuri, J Lee, A Rojiani, V Kota. Georgia Regents Univeristy, Augusta, GA; Georgia Regents University, Augusta, GA.

Background: AAM have a higher incidence \& mortality from PAC than Caucasian Men (CM), but the biological basis for these differences are poorly understood. To better understand the molecular mechanisms of this aggressive PAC in AAM, we analyzed 34 PAC resection specimens (17 each).Recently, a class of noncoding RNAs called miRNAs has emerged as critical gene regulators in cell growth, disease, and development. The goal of the current study was to evaluate the similarities and differences in PAC among AAM and CM.

Design: Archival blocks with slides were retrieved, reviewed and clinical information was obtained. Ten $10 \mu \mathrm{m}$ sections of each case were used with $>50 \%$ lesion. The miRNA expression profile of PAC ( $\mathrm{AAM}=3$ and $\mathrm{CM}=3$ ), was evaluated using the Affeymertrix miRNA microarray platform on GeneChip miRNA 3.0 array in paraffin-embedded samples. Following hybridization and data acquisition, we used Partek Genomics Suite software for RMA normalization and to determine statistically significant differences in miRNA expression between experimental groups by ANOVA and pairwise comparisons (two-sided $=0.05$ ). Based on the array data and published PAC onco-miRNAome a 38 miRNA panel was designed to be run on HTG's Super Capella on all 34 specimens. Results: The Affeymertrix 3.0 miRNA expression profile of PAC (AAM vs CM) showed significant $(>1.5$ times, $\mathrm{p}<0.05)$ upregulation of a set of 11 miRNAs, and down regulation of 10 miRNAs.
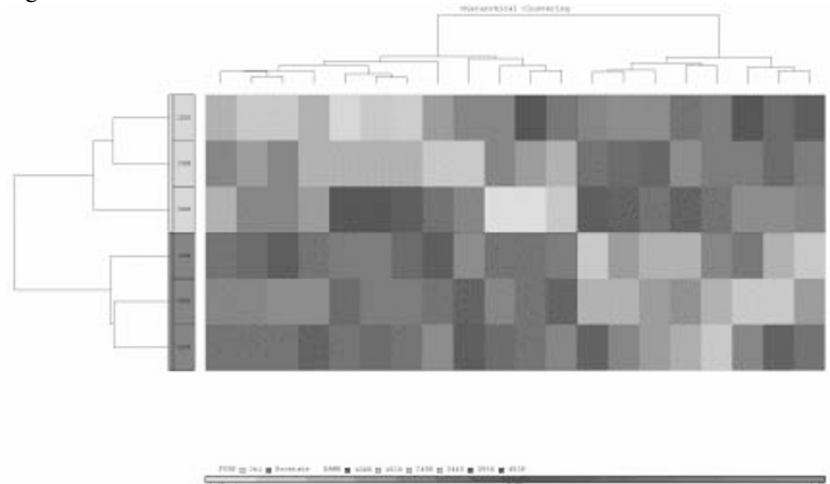

The extended HTG array showed a statically significant clinically relevant set of tumor suppressor miRNA's (mir-let7c, mir-26a and mir-145) decreased in AAM vs CM. Conclusions: We know by target prediction methods that 3' untranslated region of the ERG mRNA is a potential target of miR-145. Recent studies shows that downregulation of miR-145 may contribute to the increased expression of most ERG splice variants and hence aggressive disease. Also, loss or reduction of let- $7 \mathrm{c}$ also leads to Ras overexpression, thus, promoting cellular growth and contributing to tumor genesis We propose that this down regulation of the tumor suppressor miRNA's in AAM may explain some of the very aggressive behavior.

991 Predicting Radical Prostatectomy Outcome: Cell Cycle Progression (CCP) Score Outperforms Primary Gleason Grade among Men with Clinical Gleason $<7$ Who Are Upgraded to Gleason 7

KA Kolquist, M Cooperberg, SJ Freedland, T Schlomm, JE Reid, S Stone, MK Brawer. Myriad Genetic Laboratories, Inc, Salt Lake City, UT; UCSF Helen Diller Family Comprehensive Cancer Center, San Francisco, CA; Durham VA Medical Center and Duke University Medical Center, Durham, NC; University Medical Center HamburgEppendorf, Hamburg, Germany; Myriad Genetics, Inc, Salt Lake City, UT.

Background: Improved prognostic markers for prostate cancer are an important part of addressing the issue of over- and under-treatment in prostate cancer. Gleason score (GS), PSA and clinical stage work well for population risk assessment but lack precision for individual patients. Concern over undergrading on biopsy is felt to be a major barrier to increasing use of active surveillance. Molecular analysis can further refine risk assessment as demonstrated by the cell cycle progression (CCP) score (Prolaris ${ }^{\mathrm{TM}}$ ) which has been shown to predict prostate cancer aggressiveness in eight separate cohorts. In these studies, CCP scores typically ranged from -2 to +3 with each one-unit increase in CCP score corresponding to approximately a doubling of the risk of the studied event (recurrence or death from prostate cancer). In the present investigation, we sought to assess how upgrading compared with CCP in predicting failure after radical prostatectomy (RP)

Design: In this study, we examined men from three cohorts (UCSF, Martini Clinic, and Durham VAMC) with clinical GS (cGS) $<7$ who had pathologic GS (pGS) $3+4$ or $4+3$. The CCP assay was performed using RP specimen from UCSF and biopsy material from the other cohorts. We compared the rates of biochemical recurrence (BCR) as predicted by pGS, CAPRA or CCP score alone versus CAPRA combined with CCP score using a Cox proportional hazards model stratified by cohort.

Results: Among the 230 men with $\mathrm{cGS}<7$ and $\mathrm{pGS}=7$ included in this analysis, $207 \mathrm{had}$ pGS $3+4$ and 57 had BCR; 23 had pGS $4+3$ and 8 had BCR. There was no difference in $\mathrm{BCR}$ based on $\mathrm{GS}(\mathrm{p}=0.8)$ or CAPRA $(\mathrm{p}=0.4)$. In contrast, CCP score alone and a predefined score combining the CCP score and CAPRA were prognostic of BCR (HR=1.82 ( $95 \%$ CI 1.32-2.52; $\mathrm{p}<0.001) ; \mathrm{HR}=1.84$ (95\% CI 1.10-3.05; $\mathrm{p}=0.021)$ respectively).
Conclusions: These data indicate that the risk of BCR is indistinguishable in men with cGS $<7$ who have pGS $3+4$ or $4+3$. However, the CCP assay does provide stratification of this risk. Tests that can predict BCR on biopsy will aid in initial therapeutic decision making.

992 mTOR Pathway Activation Characterizes a Subset of Unclassified Renal Cell Carcinoma: Immunohistochemical, Molecular and Histologic Analysis

MX Kong, ARR Brannon, $H$ Won, A Gandhi, HA Al-Ahmadie, SW Fine, A Gopalan, $P$ Russo, MF Berger, SK Tickoo, VE Reuter, Y-B Chen. Memorial Sloan-Kettering Cancer Center, New York, NY.

Background: Targeted agents inhibiting the mammalian target of rapamycin (mTOR) have emerged as powerful tools in treating advanced renal cell carcinoma (RCC), particularly clear cell RCC. Limited data have also suggested efficacy of mTOR inhibitors in some non-clear cell RCC. However, predictive markers for using these agents in RCC are still lacking. High-grade unclassified RCC is a heterogenous group of aggressive non-clear cell tumors, for which there is no current standard of care. To better stratify these tumors for available agents and future clinical trials, we examined the mTOR and related pathways in a cohort of high-grade unclassified RCC.

Design: We performed immunohistochemistry (IHC) of mTOR downstream targets p-S6 and p-4EBP1 in tissue microarrays constructed from a cohort of 87 highgrade unclassified RCCs resected at our institution between 1989 and 2011. The immunoreactivity of p-S6 and p-4EBP1 was assessed using H-score [intensity $(0-3+) \mathrm{x} \%$ of positive cells]. A cut-off score of $>140$ was used to represent diffuse and moderate $(2+)$ to strong $(3+)$ staining. DNA from frozen or formalin-fixed-paraffin-embedded tissues of tumor and matched normal was analyzed in 72 cases by a targeted next-generation exome sequencing platform that evaluates mutations and copy number alterations in over 270 cancer related genes.

Results: Among 87 tumors evaluated by IHC, 27(31\%) demonstrated diffuse staining (H score $>140$ ) for $\mathrm{p}-4 \mathrm{EBP} 1$ and/or p-S6. The majority (78\%) of cases in this subset showed concordant staining for both. The remaining 60 tumors either showed weak/ patchy staining $(37 \%)$ or were negative $(32 \%)$. Targeted sequencing revealed 19 of $72(26 \%)$ tumors with PI3K/AKT/mTOR pathway mutations that involved TSC1/2 (7 cases), MTOR (5), PTEN (4), RICTOR (2) and PI3KRI (1) genes in a mutually exclusive fashion. Of the 20 tumors with strong p-4EBP $1 / \mathrm{p}-\mathrm{S} 6$ staining and available sequencing data, $11(55 \%)$ harbored one of these mutations. The remaining 9 had mutations of other genes and/or copy number alterations. Histologically, tumors with strong p-4EBP1/p-S6 staining were often high-grade with eosinophilic cytology and compact nests, tubules, solid and papillary architecture.

Conclusions: Our study suggests mutations of the PI3K/AKT/mTOR pathway are likely driving events leading to mTOR pathway activation in a subset of high grade unclassified RCCs. Other mechanisms of inducing this pathway may also exist. Activation of mTOR pathway in some unclassified RCCs provides a rationale for clinical trials evaluating therapeutic agents that target mTOR pathway.

\section{Morphologic Spectrum of So-Called Clinically Neuroendocrine} Prostate Carcinoma

M Kossai, J Fontugne, K Park, B Robinson, H Beltran, MA Rubin, JM Mosquera. Weill Medical College of Cornell University, New York, NY; Weill Medical College of Cornell University and New York Presbyterian Hospital, New York, NY.

Background: A highly aggressive variant of advanced castrate resistant prostate cancer (CRPC) with neuroendocrine differentiation is clinically referred to as neuroendocrine prostate cancer (NEPC), anaplastic prostate cancer or small cell carcinoma. With the introduction of new potent anti-androgen agents, its incidence is rising. This variant is under-recognized, notably because of lack of biopsy diagnosis for advanced stages of prostate cancer $(\mathrm{PCa})$. Given the significant therapeutic implications (hormonal vs. chemotherapy) of diagnosis of NEPC, pathologists are called to recognize the morphologic spectrum and its clinical context.

Design: We sought to characterize the histomorphology of 68 tumors from 67 patients: 46 prostate tumors (TURP and salvage prostatectomy) and 22 metastases. All patients had clinical phenotype of NEPC, i.e. rapid progression with visceral metastases and/or lytic bone lesions, occurring at variable intervals after anti-androgen therapy, associated with undetectable or low PSA. We performed histological examination, IHC and FISH and correlated results with clinical data. Cases of de novo NEPC were not included. Results: Histopathology of NEPC comprised 19 pure neuroendocrine carcinomas (12 small cell carcinoma (SCC) and 7 large cell neuroendocrine carcinoma), 3 mixed SCC/adenocarcinoma, 29 poorly differentiated carcinomas with neuroendocrine differentiation (NED) and 17 without NED. Mean age at diagnosis was 60.6 years and mean time interval to progression to castrate resistance was 5.8 years. With exception of one patient who is alive after 13 months of diagnosis of NEPC, overall survival was 12 months. $4 \%$ of poorly differentiated carcinomas tested co-expressed both AR and NE markers. $36 \%$ cases had PTEN deletion and $47 \%$ cases harbored ERG rearrangement. Conclusions: The histologic spectrum of so-called NEPC in the clinic may be the morphologic portrayal of transdifferentiation. Large cell neuroendocrine carcinoma morphology is not uncommon in the setting of clinical NEPC. There are ongoing efforts to reach consensus classification of this clinically relevant variant of aggressive treated $\mathrm{PCa}$. Unbiased next generation sequencing will provide a deeper understanding to further catalogue NEPC. 


\section{Biopsy Criteria for Determining Appropriateness for Active} Surveillance in the Modern Era

ON Kryvenko, JI Epstein. University of Miami Miller School of Medicine, Miami, FL; The Johns Hopkins Medical Institutions, Baltimore, MD.

Background: In 1994, Epstein et al, first reported criteria for identifying potentially biologically insignificant tumors that might be managed by active surveillance (AS). Criteria included: 1) PSA density (PSAD) $\leq 0.15 ; 2$ ) biopsy Gleason score $\leq 6 ; 3$ ) $<3$ positive cores: and 4 ) $\leq 50 \%$ involvement in any single core. In this study, the median number of cores sampled was 5 and $>95 \%$ of men were Caucasians. The current study assesses the Original and variations of Epstein Criteria in biopsy specimens obtained by current extended sampling techniques, using the updated Gleason grading system, and examing the criteria in African American (AA) men as well as a larger number of Caucasians.

Design: 510 (410 Caucasian, 100 AA) men with PSA $<10$ ng/dl, T1c, 12-core biopsy, \& Gleason $3+3=6$ were categorized into training and validation sets. 8 biopsy algorithms were tested for predicting insignificant $(0.5 \mathrm{cc}$, organ confined, Gleason score $<6)$ cancer at RP. Cancers incorrectly predicted to be insignificant were ranked into 4 groups of increasing aggressiveness.

Results: Original and Modified Epstein Criteria (Gleason score $<6$, PSAD $<0.15,<3$ positive cores, unilateral cancer) had the highest negative predictive values - correct classification of insignificant cancer. Increasing the number of positive cores to $>2$ led to a sizeable increase in the number of significant cancers being misclassified. Among cancers predicted to be insignificant in Caucasians, $29.9 \%$ cases using the Original and $27 \%$ cases using the Modified Epstein Criteria, had significant cancer at RP. However, more adverse findings at RP were misclassified as insignificant in only $3.5 \%$ and $2.2 \%$ of cases using the Original and Modified Epstein Criteria respectively. Of cancers predicted insignificant in AA men, $54.1 \%$ cases using the Original and $51.6 \%$ cases using the Modified Epstein Criteria were misclassified as insignificant. Dominant anterior tumors were seen in $28.5 \%$ (117/410) of Caucasians and $44 \%(44 / 100)$ of AA men.

Conclusions: The Epstein Criteria maintain their accuracy in the modern era with extended biopsy sampling. The negative predictive values are lower in AA men, in part due to higher frequency of anterior tumors. Advantages to using bilateral cancer are: 1) measuring \% core involvement can vary amongst pathologists depending on whether intervening benign prostate tissue is included in the measurement; 2) one cannot accurately quantify \% core involvement in a fragmented specimen; and 3 ) the $\%$ core involvement may vary with different core lengths.

995 Hemangiomas in Kidney with End-Stage Renal Disease - A Novel Clinicopathological Association

ON Kryvenko, SL Haley, SC Smith, SS Shen, S Paluru, NS Gupta, M Jorda, JI Epstein, MB Amin, LD Truong. University of Miami, Miami, FL; The Houston Methodist Hospital, Houston, TX; Cedars-Sinai Medical Center, Los Angeles, CA; Henry Ford Hospital, Detroit, MI; The Johns Hopkins Medical Institutions, Baltimore, MD.

Background: End stage renal disease (ESRD), with or without acquired cystic kidney disease (ACKD), is known to bear an increased risk of renal cell carcinoma. Herein, we report on a novel and distinctive association of renal hemangioma with ESRD.

Design: We studied hemangiomas in 18 kidneys with ESRD from 15 patients ( 9 months to 68 years old) from five large tertiary care institutions.

Results: ESRD was due to hypertension (4), lupus nephritis (3), focal segmental glomerulosclerosis (3), diabetes (1), IgA nephropathy (1), hereditary nephritis (1), congenital nephrotic syndrome (1), and unknown cause (1). Fourteen patients were on dialysis (4 months to 30 years). Hemangiomas were detected by surveillance imaging (6), incidentally (4), hematuria (2), flank pain (2), unknown (1). Imaging revealed heterogeneously enhancing masses. Bilateral hemangiomas were noted in 3 patients. Tumors measured 0.2 to $3.5 \mathrm{~cm}$. Twenty-nine hemangiomas were studied which appeared as a single mass (15), 2 masses (1), 4 masses (1), and 8 masses (1) per kidney. Five hemangiomas were in the renal sinus fat. Other tumors were in the medulla and often abutted on renal sinus fat. All except one of the tumors were anastomosing hemangioma showing isolated or interconnected sinusoidal capillary-sized vascular channels lined by a single layer of benign cuboidal CD34+, CD31+, D2-40- endothelial cells, separated by loose stroma with spindle cells. One tumor was cellular capillary hemangioma. Intravascular growth was seen in 7 specimens. None demonstrated necrosis or increased mitoses. All hemangiomas had extramedullary hematopoiesis. The tumors were circumscribed, varied from unencapsulated to completely encapsulated, and did not show invasion into perirenal fat. Renal sinus fat invasion and entrapment of renal tubules were noted in some cases. ACKD was seen in 10 kidneys (8 patients), renal cell carcinoma (RCC) in 4, ACKD-associated RCC precursor lesions in 3, Wilms' tumor in 1 , and papillary adenomas in 2 .

Conclusions: Anastomosing hemangioma appears as a distinctive clinicopathologic entity which develops in kidneys with ESRD, with or without ACKD. This association thus should be recognized and call for a thorough diagnostic work up of a newly diagnosed renal mass in a patient with a chronic kidney disease awaiting renal transplantation.

996 Levels of MYC mRNA and Protein Correlate in Prostate Cancer: A CISH - IHC Comparison Study

I Kulac, J Hicks, $Q$ Zheng, H Fedor, AM De Marzo. Johns Hopkins University, Baltimore, MD.

Background: Correlating levels of a given mRNA and its encoded protein has been difficult to carry out in tissues due the laborious and non-standarized nature of chromogenic in situ hybridization assays. While MYC mRNA and protein have both been shown to be overexpressed in human prostate cancer, it is not known whether MYC mRNA and protein correlate with each other. This is of particular interest given that there are a number of mechanisms to regulate MYC protein post-translationally and the mechanisms responsible for MYC overexpression in prostate cancer have not been determined.

Design: We constructed two tissue microarrays from a total 35 cases from RRPs from the Johns Hopkins Hospital. Gleason score ranged from 6-9 and pathological stage from $\mathrm{T} 2$ organ confined to T3bN1 disease. We used the ACD RNAScope 2.0 technology to perform in situ hybridization for MYC mRNA and a previously validated IHC assay to determine MYC protein levels (Mod Pathol. 2008 Sep;21(9):1156-67) on adjacent TMA sections. There were a total of 130 cores of adenocarcinoma, 170 cores of normal epithelium and 10 cores of prostate atrophy evaluated. TMAs were scanned, imported into the TMAJ database and image analysis was performed with FRIDA. Brown chromagens were used for both mRNA and protein staining and we calculated a staining score by summing the intensity of brown staining and dividing by the areas of brown staining + blue staining (hematoxylin counterstained nuclei) and determined whether mRNA and protein levels correlated.

Results: Expression of MYC at the mRNA and protein levels was higher in carcinoma than normal tissue (ttest, $\mathrm{p}<0.0001$ for RNA and 0.007 for protein). In carcinoma, there was a significant correlation between MYC mRNA and protein scores (correlation coefficient $=0.35, \mathrm{p}<0.0001)$. In atrophy, there was also a significant correlation between mRNA and protein (correlation coefficient $=0.92, \mathrm{P}=0.0002$ ). Interestingly, while MYC mRNA and protein levels were readily detected in normal epithelium, there was no significant correlation.

Conclusions: MYC mRNA and protein correlate at the tissue level in prostatic adenocarcinoma, suggesting that a primary mechanism of MYC regulation in prostate cancer occurs at the mRNA level. The overall correlation coefficient was moderate, implying that there is regulation of MYC protein levels independent of mechanisms regulating MYC mRNA levels. The combined use of ACD RNAScope for mRNA detection and protein detection by IHC using TMAs is an excellent method to determine the overall correlation of expression of mRNAs and proteins in routine pathology specimens.

\section{TERT Promoter Mutations in Urothelial Carcinoma and Potential} Clinical Applications

B Kurtis, P Zhuge, DY Zhang, C Ojaimi, JT Fallon, $M$ Zhong. Westchester Medical Center, Valhalla, NY; New York Medical College, Valhalla, NY; Mount Sinai School of Medicine, New York, NY.

Background: It is well accepted that telomeres protecting the ends of chromosomes are centrally involved in the capability for unlimited proliferation, a hallmark of cancer. Increased telomerase activity is associated with almost all types of advanced human cancer with unknown mechanism(s). Two recurrent point mutations in the promoter region of telomerase reverse transcriptase (TERT) - the key subunit of telomerase have recently been identified in melanoma as well as in a small sample set of bladder cancer cell lines. However, the incidence and clinical-pathological significance of these mutations in urothelial carcinoma have not been established yet.

Design: We collected 57 specimens of urothelial carcinoma from the upper and lower urinary tract comprising high grade and low grade; invasive and non-invasive; and primary and metastatic. We also collected matched benign tissue and 10 samples of non-urothelial carcinoma lesions. Additionally, we collected urine samples from patients with biopsy-confirmed low grade urothelial carcinoma and negative urine cytology as well as blood samples from patients whose cystectomy showed histologic evidence of extensive lymphvascular invasion. All specimens were subject to PCR amplification and bidirectional Sanger sequencing for the TERT promoter mutations: C228T and C250T. Results: 1. Carcinoma samples harbored one of the two TERT promoter mutations in $42 / 57$ samples $(74 \%$; C228T $n=35, \mathrm{C} 250 \mathrm{~T} n=7)$; the mutations were mutually exclusive of one another.

2. Mutation prevalence was roughly equal regardless of histologic grade, invasion status, or site of origin.

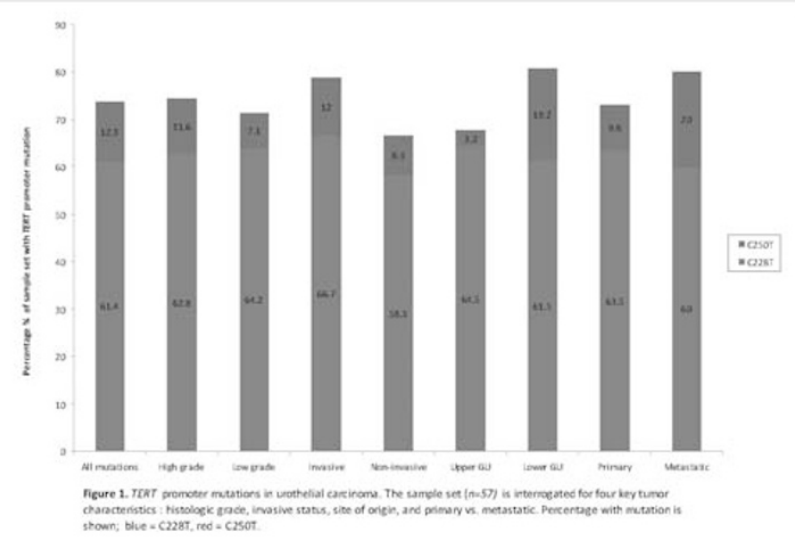

3. All matched benign and non-urothelial carcinoma samples showed wild-type sequence.

4. These mutations can be detected in urine and blood samples.

Conclusions: 1 . These TERT promoter mutations are the most common genetic alterations in urothelial carcinoma.

2. TERT promoter mutations in urothelial carcinoma are not associated with tumor location, grade or invasiveness. 
3. Detecting TERT promoter mutations in urine and blood samples may have important clinical applications in terms of patient management.

\section{IHC Helps to Identified Renal Tumors Associated with HLRCC Syndrome}

K Lara Otero, MW Linehan, MJ Merino. National Cancer Institute, Bethesda, MD; NCI, Bethesda, MD.

Background: In last years our knowledge and understanding of renal cancerpredisposing syndromes has improved significantly, and many of the genes associated with specific tumors have been discovered. Many of the tumors associated with hereditary syndromes have characteristic morphology, however, there are no specific IHC stains to assist in the identification of each tumor type. Mutations in the Fumarate Hydratase gene are associated with aggressive forms of kidney cancer. We investigated if IHC staining for the FH protein in renal cell cancer can assist in identifying renal tumors and patients part of the HLRCC syndrome.

Design: One hundred and twelve renal tumors representing the wide histologic spectrum of RCC were included in our study: 27 HLRCC tumors, 45 clear cell ( 25 with hx of VHL), 7 chromophobe, 5 Oncocytomas, 5 hybrid tumors, 3 SDHB, 2 medullary ca, 4 TFE3, 10 papillary type I, 2 Wilms and 2 TSC cancer. To evaluate FH protein expression, immunohistochemistry was performed using a mouse monoclonal antibody against human FH protein (Clone J-13, Santa Cruz Biotechnology)

Results: Eighty five (85/85) renal tumors of different subtypes (oncocytomas, ccRCC, medullary, chromophobe, SDHB, Wilms, hybrid, TSC and papillary type) were positive with cells showing strong cytoplasmic staining, indicating presence of the enzyme. Normal renal tissue, predominantly tubules also stained with marked intensity and was used as a internal control. Twenty five of 27 HLRCC related cancers showed negative staining in the tumor cells but positive adjacent renal parenchyma. Early or incipient cystic HLRCC lesions were also negative. Two cases showed equivocal results with focal areas of positivity. These 27 cases showed morphologic features consistent with HLRCC tumors.

Conclusions: Ninety three $\%$ of renal cancers part of the HLRCC syndrome, stained negative for FH by IHC meanwhile all the different types of RCC, were positive. Thus, staining for FH expression by IHC seems to be a valuable tool to identify renal tumors that may be associated with FH mutation and HLRCC syndromes. Confirmation of the syndrome by genetic testing should be performed in all cases.

\section{Array CGH Analysis of 64 Penile Squamous Cell Carcinomas:} Correlation with Pathological and Aetiological Factors

$S$ La-Touche, C Lemetre, E Stankiewicz, CK Ng, B Weigelt, $M$ Lambros, R Rajab, B Tinwell, C Corbishley, $N$ Watkin, JS Reis-Filho, DM Berney. Bart's Cancer Institute, London, United Kingdom; Memorial Sloan-Kettering Cancer Center, New York, NY; Institute of Cancer Research, London, United Kingdom; St. George's Hospital, London, United Kingdom.

Background: Penile squamous cell carcinoma (PSCC) is a rare disease and little is known about its molecular genetics. We sought to investigate the patterns and type of gene copy number aberrations in a large series PSCCs to investigate their correlations with type, and grade of tumor and also any differences between HPV positive and negative tumors.

Design: We identified 70 formalin fixed paraffin embedded PSCCs. The cases were reviewed by a panel of specialist Penile Histopathologists for grade, stage and subtype. DNA was extracted using phenol-chloroform methods from samples microdissected under a stereomicroscope. The presence of HPV DNA was assessed by broad spectrum HPV PCR using SPF10 primers. Cases were genotyped for HPV using INNO-LiPA line probe assay (Innogenetics NV,Ghent, Belgium). Gene copy number aberrations were defined using a $32 \mathrm{~K}$ BAC array platform. Copy number aberrations were described as gains, losses, amplifications or deletions. Low-level gain was defined as a cbs-smoothed Log2 ratio of between 0.08 and 0.45 and gene amplification was defined as having a Log2 ratio $>0.45$

Results: Of the 70 PSCC cases, 64 had suitable profiles for analysis. Histopathological subtypes included 50 usual type, 7 basaloid and 7 verrucous. The majority of cases were grade 3 (41), followed by grade 2 (17) and grade 1 (6). 35 Cases were HPV DNA positive and 29 HPV DNA negative. PSCCs were shown to display heterogeneous patterns of gene copy number aberrations. Gains were observed in chromosomes 1p13.3-q44 (88\%), 3p12.3-q29 (86\%), 5p15.33-p11 (67\%), 8p12-q24.3(84\%) and 11p12.3-q14.1 (39\%). Amplification of 5p15.33-p11 and 11p14.1-p12 was found in seven (11\%) and four $(6 \%)$ cases, respectively. Losses were observed in chromosomes $2 \mathrm{q} 33-\mathrm{q} 37.3(86 \%)$, 3 p26.3-q11.1 (83\%) and 11q12.2-q25 (81\%). No homozygous deletions were noted on any chromosomes. Potential candidate driver genes were identified in areas of gain (For example, PIK3CA maps to the region 3p12.3-q29, SKP2 to 5p15.33-p11 and CPT1A to 11q12.2-q25) and in areas of loss. No significant correlation was found between copy number aberration and HPV status, subtype, grade, stage or lymph node involvement. Conclusions: Although previous studies have eluded to HPV and non HPV pathways responsible in the pathogenesis of PSCC, our data demonstrates its heterogeneity, suggesting a final common molecular pathway in the evolution of all PSCCs.

1000 Characterization of Flat Urothelial Lesions with Emphasis on Interobserver Concordance and Utility of Immunohistochemical Profiling ME Lawless, MS Tretiakova, LD True, F Vakar-Lopez. University of Washington, Seattle, WA.

Background: Although criteria of urothelial neoplasms were defined by the ISUP/ WHO in 2004 publications, there is overlap in the features of the spectrum of flat intraurothelial lesions. A panel of immunohistochemical markers (CK20, p53, and CD44) can be used to more accurately characterize flat intraurothelial lesions. The aim of this study is to assess interobserver agreement (IA) in diagnoses between general pathologists and specialized genitourinary (GU) pathologists, and to compare these diagnoses with those based on IHC phenotyping.

Design: Bladder biopsies of 125 patients with flat intraurothelial lesions with atypia were classified into one of four categories: reactive atypia (RA), atypia of uncertain significance (AUS), low-grade dysplasia (LGD), and carcinoma in-situ (CIS), based on initial diagnoses by general surgical pathologists. The H\&E-stained slides were blindly reviewed by two GU pathologists. Forty-three cases, with roughly equal representation in each initial diagnostic category, were analyzed by IHC (CK20, p53, and CD44) and were blindly scored by two GU pathologists. The degree of agreement was determined using kappa statistics.

Results: Mean IA by H\&E of 125 cases with the four diagnostic categories was $63 \%$ $($ kappa $=0.47)$. IA between the two GU pathologists was $62 \%($ kappa $=0.46)$ and was similar to the mean IA and agreements of GU pathologists and general pathologists. The concordance between the GU and general pathologists was best in the RA and CIS categories and was poor in either AUS or LGD. In the 43 cases with IHC, H\&E diagnoses made by GU pathologists were more likely to be concordant with final diagnoses after IHC than those initially made by general surgical pathologists (62\% vs. $49 \%)$. Four diagnoses of CIS were rendered after interpretation of IHC that were underdiagnosed by general surgical pathologists; 3 of these cases were diagnosed as CIS by at least one GU pathologist on H\&E review. Both GU pathologist review and immunophenotyping confirmed the interpretation of all lesions initially diagnosed as CIS.

Conclusions: IA is moderate, at best, in distinguishing among the four diagnostic categories of flat intraurothelial lesions with atypia by H\&E examination alone. Concordance is similar among GU pathologists compared to general surgical pathologists. However, review by a GU pathologist and additional IHC may identify cases of carcinoma in-situ that were originally underdiagnosed and help to categorize AUS and LGD into more specific diagnostic categories.

\section{Survival Analysis in Patients with Papillary Renal Cell Carcinoma} (PRCC): Assessing the Utility of Type 1 and Type 2 Tumor Subtyping

RA Ledezma, E Negron, Z Steinberg, GP Paner, SE Eggener. University of Chicago, Chicago, IL.

Background: The prognostic factors and oncological outcomes of PRCC have not been extensively studied and the value of PRCC histologic subtyping as a predictor of outcome is somewhat controversial. Further, the recent ISUP Vancouver modification of WHO (2004) classification of kidney tumors has expanded the RCC categories and some of the newer entities may exhibit tubulo \&/or papillary architectures; tumors that may be categorized previously as PRCC. Our aim was to investigate the overall mortality in PRCC and to determine the prognostic factors for recurrence and mortality in a large surgical cohort.

Design: After obtaining IRB approval, we queried our nephrectomy database to identify patients who underwent radical or partial nephrectomy between January 2002 and December 2012. Pathologic confirmation as PRCC was performed by 1 of the authors. The primary end point (PE) of the study was overall mortality. The secondary end point (SE) was recurrence-free survival (RFS). The evaluation of PE and SE was conducted using Kaplan-Meier estimates. Cox proportional hazard regression models were used to assess predictors of mortality and recurrence.

Results: We identified 106 patients with PRCC among 1,310 patients with renal neoplasms who underwent surgery. Median follow-up was 22 months (ICR: 7.1-43.6). Eleven patients died $(10.4 \%)$ and six experienced recurrence $(5.7 \%)$. Eighty percent of our patients were pT1 and $92.4 \%$ had organ-confined disease (pT2b or lower). The 5 -year RFS was $89.5 \%(95 \% \mathrm{CI}=75.3-95.7)$. The 5 -year overall survival (OS) estimate was $86.9 \%(95 \% \mathrm{CI}=73.3-93.8 \%)$. The estimated OS and RFS was not different when stratified by type (Log rank test; $p>0.05$ ). In univariate analysis, PRCC subtype was not predictor of OS or of RFS. TNM stage group was the strongest predictor of recurrence $(\mathrm{HR}=40, \mathrm{p}<0.001)$

\section{Kaplan-Meier overall survival by PRCC type}

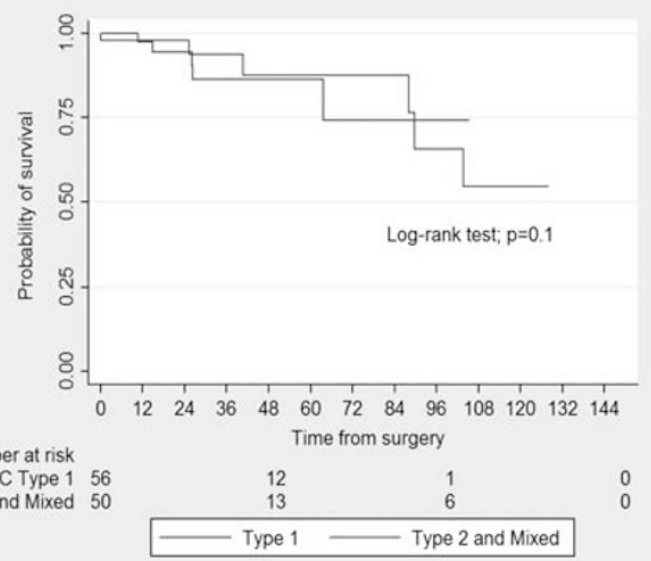

Conclusions: In concordance with prior studies, patients with PRCC have low risk of recurrence and mortality after surgery. However, we did not find differences in OS and PFR between PRCC type 1 and type 2 in our cohort. 
1002 Analysis of SPOP Mutation and Its Relationship with TMPRSS2-ERG Fusion in Prostate Cancer

B Lee, $N$ Yoon, Y-L Choi. Samsung Medical Center, Sungkyunkwan University School of Medicine, Seoul, Republic of Korea.

Background: Speckle-type POZ protein, SPOP, mediates $S R C-3$ oncogene ubiquitination and proteolysis in prostate cancer. $E R G$ is a key oncogene and approximately half of the localized prostate cancer cases harbor the TMPRSS2-ERG fusion. We performed $S P O P$ mutation analysis and investigated the relationship between $S P O P$ mutation and TMPRSS2-ERG fusion.

Design: We selected 109 cases of surgically excised prostate cancer at Samsung Medical Center in Seoul, Korea from 1995 to 2006. We performed ERG immunohistochemistry and fluorescence in situ hybridization on the tissue microarray blocks and quantitative real-time polymerase chain reaction on paraffin-embedded tissue for detection of TMPRSS2-ERG fusion and ERG expression. We divided samples into two groups: 1) 59 cases with neither TMPRSS2-ERG fusion nor ERG overexpression and 2) 50 cases with TMPRSS2-ERG fusion or ERG overexpression. All cases were sequenced by Sanger sequencing to analyze $S P O P$ somatic mutations in paraffin-embedded tissue. Results: Three missense mutations, p.Phe102Cys in one case and p.Phe125Val and p.Glu145 Lys in another case, were identified in the absence of TMPRSS2-ERG fusion and ERG overexpression $(3.39 \%, 1.83 \%$ in total). The two cases with SPOP mutations did not show distinctive clinicopathologic characteristics compared to the cases without SPOP mutation.

Conclusions: We found three missense mutations in SPOP; p.Phe102Cys and p.Phe125Val have been previously reported in the literatures $</$ del $>$; however $<$ ins datetime="2013-09-18T14:47" cite="mailto:katiem" >,</ins $>$ p.Glu145 Lys was newly discovered in this study. The mutation rate of this study was lower than that in the published data (6-13\%). The SPOP mutation was identified in only two cases, neither of which had TMPRSS2-ERG fusion or ERG overexpression. There were no cases of $S P O P$ mutation in the group with TMPRSS2-ERG fusion or ERG overexpression. This result supports a mutually exclusive relationship between SPOP and TMPRSS2-ERG fusion, as previously described.

\section{Uroplakin II Is More Sensitive Than Uroplakin III in Urothelial} Carcinoma and Its Variants

WLi, YL Liang, MT Deavers, B Czerniak, CC Guo. UT MD Anderson Cancer Center, Houston, TX

Background: Uroplakins are protein components of urothelial plaque, the hallmark of terminal urothelial differentiation. Uroplakin II (UII) and uroplakin III (UIII) are highly specific markers for urothelial differentiation. Here we evaluated the sensitivity of UII and UIII immunohistochemical staining in conventional and variant urothelial carcinomas.

Design: Immunohistochemical staining of UII and UIII was performed on tissue microarray slides, including 124 conventional bladder urothelial carcinoma (BUC), 147 variant BUC (47 micropapillary, 26 small cell carcinoma, 19 plasmacytoid, and 55 sarcomatoid variants), and 98 upper urinary tract urothelial carcinoma (UUTUC). Tumors in which $>5 \%$ of cells stained positive for UII or UIII were recorded as positive. Fisher's exact test with a two-tailed p value was used for the statistical analysis.

Results: UII expression was significantly higher than UIII expression in conventional BUC $(\mathrm{p}<0.001)$ and UUTUC $(\mathrm{p}=0.045)$.

Table 1 Uroplakin II and III expression in conventional urothelial carcinomas

\begin{tabular}{|c|c|c|c|c|c|}
\hline UC location & UII positive & UII negative & UIII positive & UIII negative & Total \\
\hline BUC & $60(48 \%)$ & $64(52 \%)$ & $23(19 \%)$ & $101(81 \%)$ & 124 \\
\hline UUTUC & $60(61 \%)$ & $38(39 \%)$ & $45(46 \%)$ & $53(54 \%)$ & 98 \\
\hline
\end{tabular}

While UII expression did not significantly differ between UUTUC and BUC $(\mathrm{p}=0.0594)$, UIII expression was significantly higher in UUTUC than in BUC $(p<0.001)$. In UC variants, UII expression was significantly higher than UIII expression in micropapillary $(\mathrm{p}<0.001)$, plasmacytoid $(\mathrm{p}<0.001)$, and sarcomatoid $(\mathrm{p}=0.032)$ variants.

Table 2 Uroplakin II and III expression in bladder urothelial carcinoma variants

\begin{tabular}{|l||l||l||l||l|l|}
\hline \hline UC variant & UII positive & UII negative & UIII positive & UIII negative & Total \\
\hline Conventional & $60(48 \%)$ & $64(52 \%)$ & $23(19 \%)$ & $101(81 \%)$ & 124 \\
\hline Micropapillary & $43(91 \%)$ & $4(9 \%)$ & $11(25 \%)$ & $36(75 \%)$ & 47 \\
\hline Sarcomatoid & $13(24 \%)$ & $42(76 \%)$ & $4(7 \%)$ & $51(93 \%)$ & 55 \\
\hline Small cell carcinoma & $1(4 \%)$ & $25(96 \%)$ & $3(12 \%)$ & $23(88 \%)$ & 26 \\
\hline Plasmacytoid & $11(58 \%)$ & $8(42 \%)$ & $1(5 \%)$ & $18(95 \%)$ & 19 \\
\hline
\end{tabular}

In the small cell carcinoma variant, only rare cases had focal UII and UIII expression, which was insufficient for analysis. Compared with conventional UC, the sarcomatoid variant had significantly lower UII expression, and the micropapillary variant had significantly higher UII expression $(\mathrm{p}<0.001)$.

Conclusions: UII is a more sensitive urothelial marker than UIII in urothelial carcinoma. The sensitivity of UII is also superior to UIII in micropapillary, plasmacytoid, and sarcomatoid variants. Although both UII and UIII are highly specific for urothelial differentiation, UII is more valuable than UIII in immunohistochemical analysis for confirming the urothelial origin.

1004 piR-32052, PiR-39894 and PiR-43607, Three Novel DicerIndependent Piwi-Interacting RNAs Derived from the Same PiRNA Cluster, Are Up-Regulated in Metastatic Renal Cell Carcinoma

$Y \mathrm{Li}, \mathrm{X} W u$, H Gao, J Jin, A Li, Y Kim, F Wang, J Wang, R Nelson, B Mu, C Guo, $J$ Wang, $W$ Chen, L Weiss, $H W u$. City of Hope, Duarte, CA.

Background: Piwi-interacting RNAs (piRNAs) are a distinct class of novel small non-coding RNAs (smRNAs) of 24-32 nucleotides in length. Forming piRNA-induced silencing complex (piRISC) with Piwi family proteins, piRNAs silence transposable elements (TEs) to protect genome integrity in the germ line across animal species. Due to their limited expression in gonads and sequence diversity, piRNAs remain the most mysterious class of smRNAs. Limited studies have shown piRNAs to be present in somatic tissues and some human cancers, however, their functional significance is still not clear. Here, we report piR-32052, piR-39894 and piR-43607 are associated with clear cell renal cell carcinoma (ccRCC) metastasis.

Design: (1) We established two separate ccRCC tissue cohorts. The 24-sample frozen tissue cohort included benign kidney tissues $(n=6)$, localized (pT1, n=6), locally extensive (pT3, n=6) and metastatic (M1, n=6) ccRCCs. The 68-formalin-fixed paraffinembedded (FFPE) tissue cohort included ccRCCs of stage I ( $n=19)$, II ( $n=5)$, III ( $n=13$ ) and IV $(n=31)$. (2) We performed smRNA sequencing (smR-seq) of the 24-specimen frozen tissue cohort and profiled the piRNA expression. (3) We examined the aberrant expression of 3 selected piRNAs in the 68-sample FFPE tissue cohort by qPCR. (4) We explored the expression of the 3 piRNAs in RCC cell lines with and without introducing antisense siRNAs of Dicer, DGCR8, Ago1, Ago2 and Ago4 genes.

Results: (1) We have shown 26,991 types of piRNAs in benign and tumorous kidney tissues by smR-seq, in which 19 piRNAs are dysregulated in tumors ( 2 up-regulated and 17 down-regulated) and 46 piRNAs are aberrantly expressed in metastatic ccRCCs (44 up-regulated and 2 down-regulated) ( $\log 2$ ratio $\geq 1, F D R \leq 0.1)$. (2) Among the piRNAs associated with metastasis, we have found piR-32052, piR-39894 and piR-43607 to be derived from the same piRNA cluster at chromosome 17. (3) We have demonstrated the expression of the 3 piRNAs not to be significantly affected when the genes of Dicer, DGCR8, Ago1, Ago2 and Ago4 were knocked down, respectively. (4) We have further validated that the overexpression of piR-32052, piR-39894 and piR-43607 are highly associated the ccRCC metastasis in the 68 -sample tissue cohort.

Conclusions: We are the first to show that piRNAs are present and differentially expressed in benign kidney tissue, localized and metastatic ccRCCs. We demonstrated that piR-32052, piR-39894 and piR-43607, originated from the same piRNA cluster and Dicer independent, to be highly associated with ccRCC metastasis.

1005 Chromophobe RCC with and without Sarcomatoid Differentiation: A Study of miRNAs in Tumor Heterogeneity and Metastasis

A Li, A Ghosh, M Alimchandani, MW Linehan, MJ Merino. National Cancer Institute, Bethesda, MD.

Background: Sarcomatoid renal cell carcinoma (sRCC) may arise from dedifferentiation in a variety of RCC subtypes including chromophobe RCC, which accounts for $5 \%$ of all renal tumors. Dysregulated miRNAs have been implicated in tumor development and metastasis and are showing significant potential in tumor detection and possible therapy. We investigated miRNA alterations in Chromophobe renal cell carcinomas with and without sarcomatoid differenciation and compared with distant metastasis to investigate to evaluate intratumor heterogeneity and a possible role in therapy.

Design: Eight cases of pure Chromophobe RCC, three with sarcomatoid dedifferentiation, as well as the spindle cell metastasis from two cases, were reviewed and the tissues of each component as well as normal kidney parenchyma were mannually microdissected. MiRNAs level was quantified using nCounter Human v2 miRNA Expression Assay.

Results: We primarily identified 15 (up reg 6 / down reg 9), 17 (up reg 10 / down reg 7 ), and 76 (up reg 55 / down reg 21) miRNAs differentially expressed in carcinoma, sarcomatoid (fold change $\geq 2$ or $\leq-2 ; \mathrm{p}<0.05$ ) and metastatic components (fold change $\geq 2$ or $\leq-2$ ) relative to normal controls in chRCC respectively. We further identified 13 aberrantly expressed miRNAs (up reg 8 / down reg 5) (fold change $\geq 2$ or $\leq-2 ; p<0.05$ ) in the sarcomatoid component relative to normal controls being able to discriminate sarcomatoid from chromophobe components. Twelve miRNAs (up reg 10 / down reg 2) were found universally deregulated in metastasis relative to carcinoma, sarcomatoid tumor, and normal controls (fold change $\geq 2$ or $\leq-2$ ). MiR-204-5p is the most differentially expressed gene and commonly deregulated in carcinoma (fold change $=-29.0 ; p=0.007$ ), sarcomatoid (fold change $=-37.0 ; p=0.024$ ), and metastatic components (fold change $=-38.3$ ), indicating this miRNA might play a critical role in all three heterogeneous components in chRCC.

Conclusions: Our study demonstrates that miRNA deregulation participates in the development of chromophobe RCC and the evolution from chromophobe carcinoma to sarcomatoid differentiation and metastasis. miRNAs may have potential as markers for diagnosis, prognosis and future therapies of Chromophobe RCC.

1006 Differential Expression of GATA-3 in Urothelial Carcinoma Variants Y Liang, J Heitzman, B Czerniak, CC Guo. University of Texas MD Anderson Cancer Center, Houston, TX.

Background: Urothelial carcinoma (UC) variants demonstrate a variety of histologic features that are distinct from conventional UC, which may present a difficulty in recognizing the urothelial origin of these variants. As GATA-3 is a new specific marker for UC, we studied its expression in different $\mathrm{UC}$ variants and compared its expression between bladder UC (BUC) and upper urinary tract UC (UUTUC).

Design: Immunohistochemical staining for GATA-3 was performed on tissue microarray slides, including 103 conventional BUC, 46 micropapillary variant, 55 sarcomatoid variant, 26 small cell carcinoma variant, 16 plasmacytoid variant, 15 squamous cell carcinoma, and 82 conventional UUTUC. Tumors were considered positive if more than $5 \%$ of tumor cells had positive nuclear staining. Fisher's exact test with a two-tailed $\mathrm{p}$ value was used for the statistical analysis.

Results: GATA-3 was expressed in 70\% (72 of 103) of conventional UC. The GATA3 expression in micropapillary ( $57 \%$ [26 of 46]) and plasmacytoid (44\% [7 of 16]) variants did not significantly differ from that in conventional UC. However, GATA-3 expression in sarcomatoid (13\% of [ 7 of 55]) and small cell carcinoma (4\% [1 of 26]) variants was significantly lower than that in conventional UC ( $<<0.001)$. Only $7 \%$ (1 of 15) of squamous cell carcinomas expressed GATA-3, significantly lower than UCs $(\mathrm{p}<0.001)$. GATA-3 expression was observed in $32 \%$ (26 of 82$)$ of UUTUCs, which was significantly less than that in bladder UCs $(\mathfrak{p}<0.0001)$. Among UUTUCs, GATA-3 
expression in the pelvis ( 7 of $26[20 \%])$ was lower than that in the ureter $(13$ of $26[45 \%])$ and ureteropelvic junction (6 of 26 [33\%]). GATA-3 expression was not significantly associated with tumor grade, tumor stage, or clinical outcome in our patient cohorts Conclusions: GATA- 3 expression differed in UC variants, was more common in micropapillary and plasmacytoid variants, and was uncommon in small cell carcinoma and sarcomatoid variants. GATA-3 immunostaining may be valuable for confirming the urothelial origin of micropapillary and plasmacytoid variants but not of sarcomatoid or small cell carcinoma variants. Because of low GATA-3 expression in UUTUC, GATA-3 immunostaining results should be interpreted with caution when used to confirm the urothelial origin of UUTUC.

1007 SLC14A1 Down-Regulation Is Associated with Poor Clinical Outcome in Patients with Urothelial Carcinoma of Upper Urinary Tracts and Urinary Bladder

$P-I$ Liang, C-F Li. Kaohsiung Medical University Hospital, Kaohsiung Medical University, Kaohsiung, Taiwan; Chi Mei Foundation Medical Center, Tainan, Taiwan; Southern Taiwan University of Science and Technology, Tainan, Taiwan; National Health Research Institutes, Tainan, Taiwan.

Background: Urothelial carcinoma (UC) is a common cancer in industrialized country. UC of urinary bladder (UCUB) and of upper urinary tract (UCUT) may share similar tumorigenesis. Previously, genome-wide association studies identified that genetic variation of solute carrier family 14 transporters, member 1 (SLC14A1) is associated with bladder cancer development. By using public domain datasets and our wellcharacterized cohort, we intended to analyze the association of SLC14A1 expression in UCUB and UCUT.

Design: Two datasets from GEO (GSE31684 and GSE32894) were selected and analyzed to examine the significance of $S L C 14 A 1$ transcript expression in relation to primary tumor (pT) status. SLC14A1 transcript levels were further validated by using Quantigene assay in 35 and 40 UBUC and UTUC cases respectively, and were further correlated with various clincopathological factors. SLC14A1 immunostain was performed on 340 cases of UCUT and 295 cases of UBUC and interpreted by using $\mathrm{H}$-score. The immunoexpression was then correlated with clinicopathological features, disease-specific survival (DSS), and metastasis-free survival (MeFS).

Results: Validation of $S L C 14 A 1$ transcript in public domain datasets showed a stepwise down-regulation during UCUB progression, which is also confirm by using Quantigene assay in our cohort. In the immunohistochemical study, loss of SLC14A1 expression in both groups of UCs significantly associated with advanced pT stage (both $p<0.001$ ), lymph node metastasis (both $p<0.001$ ), high histological grade (both $p<0.001$ ), vascular invasion (both $p<0.001$ ), perineurial invasion (UCUT, $p=0.002$; UCUB, $p=0.021$ ), and frequent mitosis (both $p=0.003$ ). Loss of SLC14A1 expression also independently predicted poor DSS (UCUT $p=0.033$; UCUB $p=0.005$ ) and MeFS (UCUT $p=0.012$; UCUB $p=0.031$ ) in both groups of patients.

Conclusions: SLC14A1 down-regulation is associated with advanced tumor stage and conferred poorer clinical outcome, justifying that SLC14A1 is a potential prognostic biomarker of UC.

1008 miRNAs Are Involved in the Formation and Maintenance of SelfRenewing Kidney Cancer Spheres with Stem Cell Properties

Z Lichner, C Saleh, V Subramaniam, A Seivright, G Prud'homme, GM Yousef. Keenan Research Centre in the Li Ka Shing Knowledge Institute, St. Michael's Hospital, Toronto, ON, Canada; University of Toronto, Toronto, ON, Canada.

Background: Cancer cells may acquire stem cell (CSC) properties by the activated TGF $\beta$-epithelial-mesenchymal transition (EMT) axis resulting in the formation of cancer stem cells. miRNAs are involved in CSC formation and maintenance in solid tumors, but theire role has not been investigated in renal cell carcinoma (RCC). Renal cell carcinoma (RCC) is an aggressive disease, with $35 \%$ chance of progressing to metastasis. Design: mRNA and miRNA expression was quantified by RT-qPCR. miRNA expression profiling was carried out on TaqMan Low Density Arrays. Cell lines were transfected with miRNA precursors or with LNA-based inhibitors. RCC cells were subcutaneously injected for the xenograft studies.

Results: We isolated RCC spheres from ACHN and CAKI-1 cell lines and showed that they exhibit cancer stem cell-like properties including formation of self-renewing spheres, high clonogenicity, high tumorigenic capacity and increased expression of epithelial-mesenchymal transition markers and stem cell-specific transcription factors. RCC sphere xenografts recapitulated kidney cancer histological characteristics. qRTPCR screening identified differentially expressed miRNAs in the RCC spheres compared to their parental cell lines. Target prediction analysis pointed out TGF $\beta$ pathway activation as a possible mechanism to drive RCC sphere formation. Our findings show that inhibition of miR-17 results in rapid formation of RCC spheres. We also validated (mRNA)TGFBR2 as a direct and biologically relevant target of miR-17 during RCC sphere formation. Further, we demonstrate that TGF-betal induces RCC sphere formation and this is hindered by miR-17 transfection or siRNA silencing of TGFBR 2 . Conclusions: Our results indicate that TGF-beta/epithelial-mesenchymal transition axis is likely a significant contributor to RCC sphere formation and that anti-miR-17 contribute to this process by releasing inhibition of $T G F B R 2$.
1009 A Novel Metric to Assess Tumor Load (TL) in the Neoadjuvant Setting: Data from a Randomized, Phase 2 Trial in Patients (pts) with Localized, High-Risk Prostate Cancer (LHRPC)

RT Lis, $H$ Ye, LD True, M Loda, $W$ Peng, T Kheoh, $N$ Tran, A Molina, PW Kantoff, $M-E$ Taplin, $P$ Troncoso. Dana-Farber Cancer Institute, Boston, MA; Beth Israel Deaconess Medical Center, Boston, MA; University of Washington, Seattle, WA; Janssen Research \& Development, Los Angeles, CA; The University of Texas MD Anderson Cancer Center, Houston, TX

Background: TL is difficult to assess in radical prostatectomy specimens (RPS), as prostate cancer (PC) infiltrates between benign glands and stroma and is present in multiple cross sections. This is especially problematic in RPS after new generation androgen deprivation therapy (ADT), as intense ADT produces tumor regression with sparse residual tumor cells scattered in a large paucicellular tumor bed. In those cases, tumor volume (TV) estimation based on tumor dimensions in the histologic sections may overestimate residual TL. Here we assessed (for the first time in PC) residual cancer burden (RCB), a novel metric defined as TV adjusted for tumor cellularity (TC), in a randomized, phase 2 neoadjuvant trial of LHRPC pts.

Design: Pts with biopsy proven LHRPC $(\mathrm{n}=56)$ were randomized to leuprolide acetate (LHRHa) or LHRHa + abiraterone acetate and prednisone on two arms, followed by RP. TC of residual tumors was assessed by blinded, central review among five pathologists. Median TC was used for calculations. TL was calculated by using 1) the largest crosssectional (CS) dimension of tumor in reconstructed 'whole CS slices', with minimal residual disease (MRD) defined as $\leq 0.5 \mathrm{~cm} ; 2$ ) RCB $=$ TV x TC, with TV calculated by the 3D volume estimation method based on the largest CS dimension of tumor and the number of CS involved by tumor, corrected for TC, with MRD defined as RCB $\leq$ $0.25 \mathrm{cc}(\mathrm{TV} \leq 0.5 \mathrm{cc} \times \mathrm{TC} \leq 50 \%)$

Results: Intense ADT resulted in paucicellular tumors in cases with good responses. TL was redistributed after using RCB as measurement tool. The number of MRD cases increased from $8(14 \%)$ by size to $31(55 \%)$ by RCB (Figures A\&B). Correlation of RCB with androgen levels and androgen pathway gene expression, as well as the predictive value of RCB, are being investigated.

Conclusions: RCB provides an alternative assessment of TL in the neoadjuvant setting. We propose that RCB be used for neoadjuvant ADT treated PC.

A

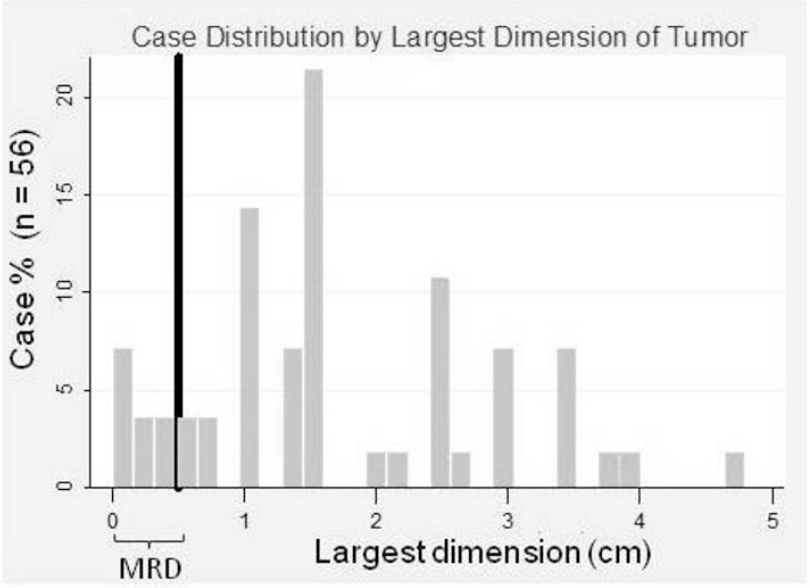

B

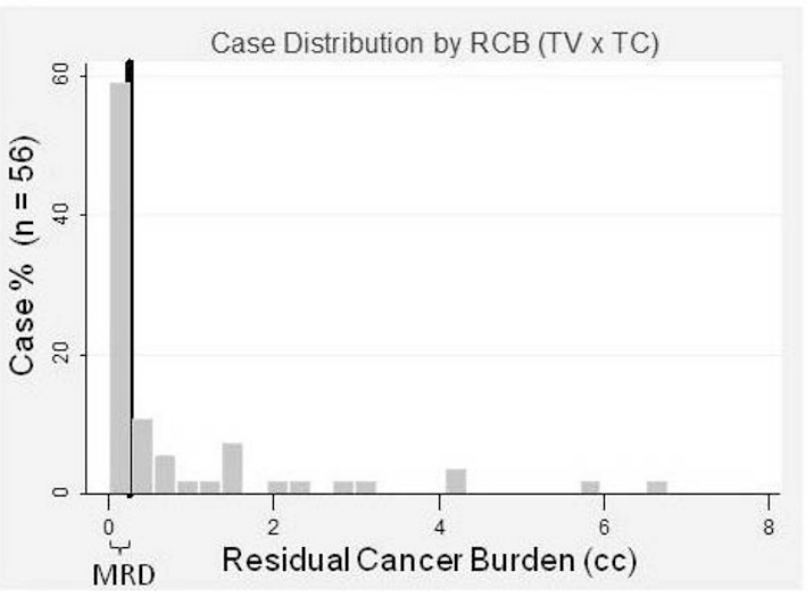


1010

Positive Margins in Partial Nephrectomy Specimens Do Not Predict Recurrence

C Liu, A Heimann, W Waltzer, S Wu, M Zawin, S Hwang, J Liu. Stony Brook Medicine, Stony Brook, NY.

Background: For selected patients with renal cell neoplasms, partial nephrectomy is the standard of care. There have been limited studies reporting the recurrence rates ranging from $0 \%$ to $27 \%$. We evaluated recurrence rates following partial nephrectomies (PN) at our institution.

Design: Retrospective data were collected from patients with renal tumors who underwent PN from January 2000 to April 2013 at a single academic medical center. Patients with less than 6 months of follow-up were excluded from this study. We evaluated the status of resection margins and tumor types in the PN specimens, as well as re-excision specimens. Urologists frequently resect tissue from the tumor base as additional margin. In this study, we analyzed the status of both margins.

Results: A total of 261 PN were performed during the time period. 208 cases are malignant renal cell neoplasms ( $80 \%)$ and 53 cases are benign renal neoplasms $(20 \%)$. Of the 261 cases, 8 had tumor in the final resection margin at the initial PN procedure ( 5 malignant and 3 benign neoplasms). There were $5(1.9 \%)$ recurrences, including 3 clear cell renal cell carcinoma and 2 papillary renal cell carcinoma. All 5 recurrent tumors have negative final margins in the initial PN procedure and are of the same tumor type as in the initial resections. There were no recurrences of the 8 tumors with positive final margins at the initial partial nephrectomies. The margin status and tumor types of the 5 recurrent cases are listed in Table 1. The interval between initial surgeries and recurrences ranged from 2.5 to 11 years ( 2.5 years, 3.5 years, 4.5 years, 5 years and 11 years).

\begin{tabular}{|c|c|c|c|c|}
\hline \\
\hline \begin{tabular}{|l|} 
Initial \\
diagnosis
\end{tabular} & $\begin{array}{l}\text { Recurrent } \\
\text { Diagnosis }\end{array}$ & $\begin{array}{l}\text { Tumor } \\
\text { margin }\end{array}$ & $\begin{array}{l}\text { Additional } \\
\text { margins }\end{array}$ & Recurrence Interval (years) \\
\hline Clear Cell RCC & Clear Cell RCC & Negative & Negative & 4.5 \\
\hline Clear Cell RCC & Clear Cell RCC & Negative & Negative & 3.5 \\
\hline Clear Cell RCC & Clear Cell RCC & Positive & Negative & 11 \\
\hline Papillary RCC & Papillary RCC & Positive & Negative & 2.5 \\
\hline Papillary RCC & Papillary RCC & Negative & Negative & 5 \\
\hline
\end{tabular}

Conclusions: Our data indicates that approximately $1.9 \%$ of patients undergoing partial nephrectomy had recurrence, despite having negative final margins at initial resection. Conversely, we found 8 cases with tumor in the final margins at initial resection, and none have recurred. Our data indicates that positive margins in partial nephrectomies do not predict recurrence and negative margins do not preclude future recurrence.

1011 ERG and PTEN Status of HOXB13 G84E-Related Prostate Cancers: An Analysis of 106 Cases

TL Lotan, M Zhang, H Fedor, J Hicks, CM Ewing, SD Isaacs, AM De Marzo, WB Isaacs. Johns Hopkins Medical Institutions, Baltimore, MD.

Background: A recurrent germline mutation (G84E) in the $H O X B 13$ gene is associated with early onset familial prostate cancer in patients of European descent, occurring in up to $5 \%$ of prostate cancer families. However, the clinical-pathologic and molecular features of prostate tumors occurring in $\mathrm{HOXB13}$ G84E carriers have not been studied in a large cohort of patients.

Design: We identified 106 heterozygous carriers of G84E who underwent radical prostatectomy for prostate cancer between 1985 and 2011. Tissue microarrays (TMAs) were prepared using four-fold redundancy of sampling of the index (highest grade/ largest size) tumor. TMAs were immunostained and scored for PTEN loss and ERG expression using genetically validated and previously published assays.

Results: Of patients with the G84E mutation, 99\% (105/106) were of European descent. Mean age at radical prostatectomy was 55 years (38-70) and median pre-operative PSA was $6.1 \mathrm{ng} / \mathrm{ml}(1-50.6) .51 \%$ (45/88) of patients did not have a family history of prostate cancer, $34 \%(30 / 88)$ had one first degree relative with prostate cancer and $15 \%(13 / 88)$ had two or more relatives (at least one first degree) with prostate cancer. At radical prostatectomy, $57.5 \%(61 / 106)$ of cases had Gleason score (GS) $3+3=6$ tumors, $24.5 \%$ (26/106) had GS $3+4=7$ tumors, $9.4 \%(10 / 106)$ had GS $4+3=7$ tumors, and $8.5 \%(9 / 106)$ had GS 8-10 tumors. 70.7\% (75/106) were pT2, 23.6\% (25/106) were pT3A, $2.8 \%$ $(3 / 106)$ were pT3B and $2.8 \%(3 / 106)$ had involvement of pelvic lymph nodes (N1). $33 \%(26 / 78)$ of patients had anterior dominant tumors. ERG was positive in at least one tissue core from the index tumor in $34.6 \%(36 / 104)$ of G84E carriers, a significantly smaller fraction than seen in genetically unselected racially diverse cohorts from our institution $(49.3 \%$ or $534 / 1083$, Fisher's exact $p=0.005)$ or genetically unselected whites $(60.8 \%$ or $45 / 74$, Fisher's exact $p=0.0007$; see Kulac et al, USCAP 2014). PTEN was lost in at least one core of the index tumor in $11.5 \%(12 / 104)$ of patients and PTEN loss was significantly more common in ERG positive tumors $(26 \%$ or $9 / 35)$ vs. ERG negative tumors $(3.0 \%$ of $2 / 67$; Fisher's exact $\mathrm{p}=0.0009)$.

Conclusions: $E R G$ rearrangement, as assessed by ERG expression, is significantly less common in surgically-treated $H O X B 13$ G84E-related prostate tumors than in genetically unselected white cohorts. PTEN loss is infrequent overall in G84E-related tumors, and occurs almost exclusively in ERG-positive tumors in this cohort.

1012 PTEN Loss Predicts Upgrading of Prostate Cancer from Biopsy to Radical Prostatectomy

TL Lotan, FL Carvalho, SB Peskoe, JL Hicks, J Good, H Fedor, E Humphreys, M Han, EA Platz, JA Squire, AM De Marzo, DM Berman. Johns Hopkins Medical Institutions, Baltimore, MD; Johns Hopkins Bloomberg School of Public Health, Baltimore, MD; Queen's University, Kingston, ON, Canada.

Background: Active surveillance is increasingly recommended for men with low risk Gleason Score $3+3=6$ (GS 6) prostate cancer, yet, approximately one third of patients with GS 6 cancer on biopsy are upgraded to higher GS at radical prostatectomy (RP).
Clinical-pathologic parameters (age, PSA, prostate volume, extent of disease) are only weak predictors of upgrading in patients with GS 6 biopsies. Here, we investigated the utility of PTEN as a molecular marker to predict upgrading in GS 6 biopsies.

Design: 71 biopsies containing GS 6 tumors that were upgraded to GS 7 or higher cancer at RP (cases) were compared to 103 GS 6 biopsies that were not upgraded at $\mathrm{RP}$ (controls). The most extensively involved needle core biopsy from each patient was evaluated for PTEN protein loss using a previously validated immunohistochemistry (IHC) assay. Fluorescence in situ hybridization (FISH) was used to assess for PTEN gene deletion in biopsies with PTEN protein loss. The correlation of upgrading with PTEN loss and with clinical-pathologic variables was assessed by logistic regression. Results: Upgraded patients were older than controls (61.8 vs. 59.3 years), had higher mean pre-operative PSA levels ( $6.53 \mathrm{vs.} 5.26 \mathrm{ng} / \mathrm{mL}$ ), and a higher fraction of biopsy cores involved by tumor $(0.42 \mathrm{vs}, 0.36)$. PTEN protein loss by IHC was found in $18.3 \%(13 / 71)$ of upgraded cases compared to $6.8 \%(7 / 103)$ of controls $(\mathrm{p}=0.02)$. In the biopsies with PTEN protein loss, FISH demonstrated homozygous PTEN deletion in $90 \%(9 / 10)$ of upgraded tumors compared to $67 \%(4 / 6)$ of interpretable controls that were not upgraded. On multivariate analyses, tumors with PTEN protein loss were significantly more likely to be upgraded at RP compared to those without PTEN loss, even after adjusting for age, preoperative PSA, clinical stage and race with odds ratio $(\mathrm{OR})=3.04(1.08-8.55 ; \mathrm{p}=0.035)$.

Conclusions: In prostate needle biopsies, PTEN IHC may help distinguish men with low risk cancer from men with intermediate or higher risk cancers and improve patient selection for active surveillance protocols.

1013 Discontinuous and Unilateral Involvement of 12 Part Core Biopsies by Adenocarcinoma Predicts Bilateral Involvement of Subsequent Radical Prostatectomy

BM Lowenthal, F Wen, N Bagherzadeh, SMahooti. The University of California at San Diego and VA San Diego, La Jolla, CA

Background: Bilateral (BI) involvement of the prostate by adenocarcinoma (AdenoCA) confers higher pathological and clinical stage disease. Screening for AdenoCA is done on core biopsies (PBX) with left and/or right sided involvement, Gleason Score (GS), and percentage of tumor (\% TUM) as the main considerations for subsequent prostatectomy and management. \%TUM in PBX is measured in two ways. One way records malignant glands in aggregate (AGG). The other way measures discontinuous involvement (DIS) of PBX by AdenoCA, taking into account the intervening stroma and benign glands between the malignant glands. This study compares the two methods as a way to predict BI involvement in subsequent radical prostatectomy (RP) specimen. Design: PBX and subsequent RP performed at our institution from 2009 to 2012 were reviewed and separated into those with unilateral (UNI) and BI involvement by AdenoCA. Sixteen cases were found to be UNI on PBX with UNI involvement on RP, while fifty-nine cases were found to be UNI on PBX with BI involvement on RP. Each case was evaluated for GS, number of cores involved, and DIS versus AGG involvement by AdenoCA independently by three pathologists. A t-test was used to determine if any of these measured variables correlated with BI involvement of the $\mathrm{RP}$ in a statistically significant manner.

Results: In UNI PBX and subsequent UNI RP, there is no statistical significance between \%TUM determination using either the AGG or the DIS method ( $p=0.4)$. In UNI PBX with subsequent $\mathrm{BI}$ involved RP there is a highly statistically significant difference $(\mathrm{p}=0.002)$ between $\%$ TUM determination using the AGG versus DIS method. There is no statistically significant difference between UNI PBX/UNI RP versus UNI PBX/BI $\mathrm{RP}$ when measuring GS ( $\mathrm{p}=0.88$ ) or number of cores involved by AdenoCA $(\mathrm{p}=0.27)$. Conclusions: Measuring \%TUM using the DIS method predicts BI AdenoCA involvement and a higher clinical pathological stage. DIS PBX involvement takes into account multifocality and may be a better predictor of BI involvement of the whole prostate even if the PBX's are only UNI positive for AdenoCA.

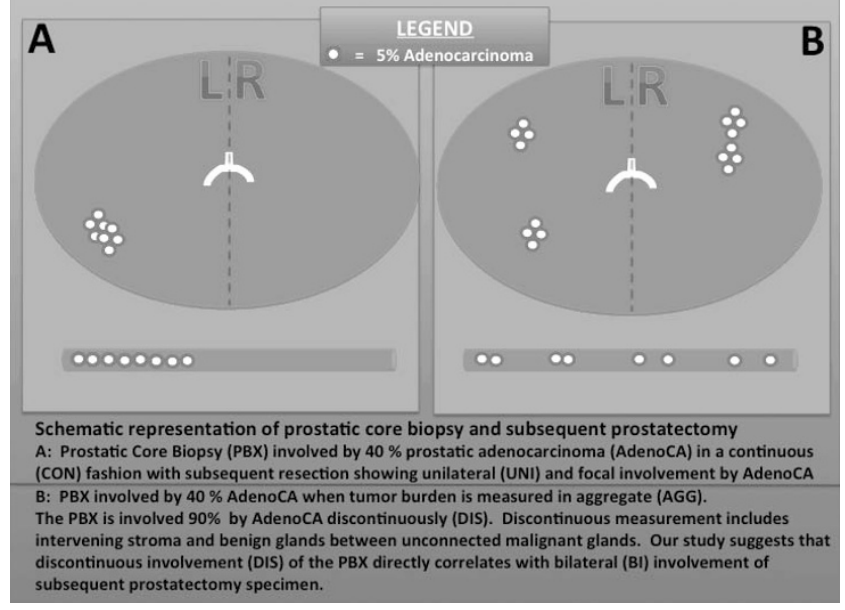


1014

\section{from Its Mimics}

Claudins 4 and 8 Help to Distinguish Metanephric Adenoma

$S$ Lu, D Treaba, S Mangray, R Monahan, C Magi-Galluzzi, M Resnick, E Yakirevich. Alpert Medical School of Brown University, Providence, RI; Cleveland Clinic, Cleveland, $\mathrm{OH}$

Background: Claudins are members of a large family of tight junction proteins, which regulate cellular adhesion, polarity, and glandular differentiation. Dysregulation of claudin protein expression has been described in a number of malignancies, including renal cell carcinoma and oncocytoma; however, expression of claudins in metanephric adenoma (MA) and its mimics including the epithelial predominant Wilms tumor (eWT) and solid variant of papillary carcinoma (s-PRCC) has not been addressed. Our goal was to investigate the differential protein expression patterns of claudins $1,3,4$, 7 , and 8 in MA, e-WT, and s-PRCC.

Design: Paraffin embedded tissue microarrays containing 17 e-WTs, 15 s-PRCCs, and 4MAs, and whole tissue sections of 14 MAs were analyzed for IHC expression of claudins $1,3,4,7$, and 8 . The immunoreactivity was assessed based on a combined score of the extent and intensity on a scale of $0-3+$.

Results: All claudins demonstrated a membranous staining pattern in both the nonneoplastic renal tissue and tumors. Nephrogenic rests were immunoreactive for claudins 1,3 , and 4 , and negative for claudins 7 and 8 . All s-PRCCs and the majority $(82 \%)$ of e-WTs stained moderately to strong $(2-3+)$ for claudin 4 , while all MAs were negative $(\mathrm{p}<0.0001$, table 1$)$. The immunoreactivity of claudin 8 was similar to that of claudin 4 with slightly lower sensitivity for s-PRCC and e-WT $(\mathrm{P}<0.0001)$. Claudins 1 and 3 were moderate to strongly positive $(2-3+)$ in all MAs and the majority of e-WTs. Within the s-PRCC group claudin 3 expression was more frequent as opposed to claudin $1(80 \%$ and $33 \%$, respectively). Expression of claudin 7 was not statistically different between the study groups.

\begin{tabular}{|c|c|c|c|c|}
\hline & MA (n=18) & s-PRCC $(\mathrm{n}=15)$ & $e-W T(n=17)$ & P value \\
\hline Claudin 1 & $18(100 \%)$ & $5(33 \%)$ & $15(88 \%)$ & $<0.000$ \\
\hline Claudin 3 & $18(100 \%)$ & $12(80 \%)$ & $16(93 \%)$ & 0.0055 \\
\hline Claudin 4 & 0 & $15(100 \%)$ & $14(82 \%)$ & $<0.0001$ \\
\hline Claudin 7 & $3(17 \%)$ & $6(40 \%)$ & $5(29 \%)$ & 0.1643 \\
\hline Claudin 8 & 0 & $13(87 \%)$ & $13(77 \%)$ & $<0.000$ \\
\hline
\end{tabular}

Conclusions: MAs characteristically express claudins 1 and 3 and are negative for claudins 4 and 8 . Claudins 4 and 8 are highly sensitive and specific markers for distinguishing MA from s-PRCC and e-WT and should be considered in the IHC panel to more accurately subtype these tumors.

\section{Renal Leiomyoma: A Multi-Institutional Study of 9 Cases}

C Magi-Galluzzi, JK McKenney, K Trpkov, L Rosen, P Gattuso. Cleveland Clinic, Cleveland, OH; University of Calgary and Calgary Laboratory Services, Calgary, AB, Canada; Rush University Medical Center, Chicago, IL.

Background: Renal leiomyoma is an uncommon benign mesenchymal tumor of the kidney, predominantly located in the renal capsule ("capsuloma") and pelvis, or in proximity of those structures. Due to their low incidence, nonspecific clinical symptoms and lack of well-defined imaging features, leiomyomas are rarely included in the differential diagnosis of solid renal masses and often represent a diagnostic challenge. Herein we report 9 cases of renal leiomyomas diagnosed at three institutions.

Design: We queried the Cleveland Clinic, Rush University Medical Center, and University of Calgary surgical pathology files for patients diagnosed with renal leiomyoma or capsuloma. Patient's age, sex, specimen type, tumor location, size and gross characteristics were collected in an IRB approved database. All slides were reviewed to confirm the diagnosis. A representative paraffin block was stained with antibodies against desmin, HMB-45, estrogen (ER) and progesterone (PR) receptors. Results: Nine renal leiomyomas were identified in 8 female patients with a mean age of 64 years (range 53 to 81 ). Tumor size ranged from $0.3 \mathrm{~cm}$ to $7.0 \mathrm{~cm}$. All lesions were distinct, white-tan, solid nodules without cystic changes. Seven tumors were solitary; in one patient with end stage renal disease, two distinct neoplasms were noted $(0.3$ and $0.4 \mathrm{~cm}$ ). Seven tumors originated from the renal capsule, one from a vessel within the renal sinus, and one was found in the proximity of the renal pelvis. Right and left kidneys were equally involved. Radical and partial nephrectomy were performed in 4 patients each, respectively. One post-transplant patient who underwent bilateral nephrectomy had a leiomyoma in the left and a clear cell renal cell carcinoma in the right kidney. Tumors were composed of tight fascicles of fusiform cells with densely eosinophilic cytoplasm, within a hyalinized stromal component. All tumors were diffusely positive for desmin and negative for HMB-45. Seven (78\%) lesions showed diffuse immunoreactivity for both ER and PR; one showed focal ER positivity. All tumors behaved in an indolent fashion.

Conclusions: Although uncommon, renal leiomyoma should be included in the differential diagnosis of solid renal masses in postmenopausal women. Since most of the cases express ER and PR, this study provides supporting evidence that sex steroid hormones may play a role in the pathogenesis of renal leiomyoma.

1016 Evaluation of a Decision Tree in the Diagnosis of Renal Neoplasms Based on Genomic Aberrations Detected by Array-CGH

C Magi-Galluzzi, CG Przybycin, JK McKenney, P Carver, K Streator Smith, E Klein, B Rini, S Campbell, C Ma, B Gowrishankar, J Houldsworth. Cleveland Clinic, Cleveland, $\mathrm{OH}$; Cancer Genetics Inc, Rutherford, NJ.

Background: Tumor genomes are unstable with gains and losses of specific chromosomal regions which may have pathologic and clinical significance. Microarraybased comparative genomic hybridization (array-CGH) has been developed to evaluate copy number alterations (CNA) in the genome. The aim of our study was to evaluate if a decision tree based on specific chromosomal regions detected by array-CGH and combinations thereof can assist in the diagnosis of different common subtypes of renal cortical neoplasms.

Design: DNA extracted from 5x10 micron sections of 190 FFPE renal tumors collected with IRB approval, was submitted to array-CGH with a sex-matched reference DNA but blinded to morphology, using a custom array representing genomic regions commonly altered in urogenital neoplasms. Gain/loss/no change information relative to the reference DNA obtained for each represented region for each specimen was then used to classify tumor subtype according to a decision tree previously optimized on CNA information obtained from TCGA, prior FISH studies, and published literature. Results: Tumors evaluated included: 63 clear cell renal cell carcinomas (CCRCC), 57 papillary RCC (PRCC), 34 chromophobe RCC (ChRCC) and 36 oncocytomas (ONC); 62 patients were female and 128 male; mean age was 61 years. 53/63 (84\%) CCRCC were accurately classified; 3 were classified as benign (no aberrations) or ONC and 4 as PRCC (one of which showed features of clear cell-papillary RCC [CC-PRCC] on review); in 3 cases tissue quality was poor. 50/57 (88\%) PRCC were accurately classified; 1 was classified as benign, 4 as CCRCC (one of which showed features of CC-PRCC on review) and 2 as not-classifiable (NC)(aberrations detected were not consistent with any subtype). 31/34 (91\%) ChRCC were correctly classified (multiple chromosomal aberrations except gain of $17 \mathrm{q}) ; 2$ cases were $\mathrm{NC}$; in one case tissue quality was poor. $29 / 36(80 \%)$ ONC were classified as benign or ONC: 12 showed no genomic imbalance and 17 had partial or complete loss of chr.1; 1 was classified as CCRCC; 6 were NC (cases had borderline nuclear features on review).

Conclusions: The decision tree applied in this study was able to accurately diagnose $91 \%$ of ChRCC, $88 \%$ of PRCC, $84 \%$ of CCRCC and $80 \%$ of ONC. After review of the tree, better typing of not-classifiable specimens may be possible and the addition of the mutation status of $V H L$ to the tree may lead to improved classification of CCRCC.

1017 Quantitative Proteomic Analysis Reveals Potential Diagnostic Markers and Pathways Involved in Pathogenesis of Renal Cell Carcinoma O Masui, NMA White, LV DeSouza, S Metias, AD Romaschin, RJ Honey, R Stewart, K Pace, J Lee, MAS Jewett, GA Bjarnason, KWM Siu, GM Yousef. York University, Toronto, ON, Canada; St. Michael's Hospital, Toronto, ON, Canada; University of Toronto, Toronto, ON, Canada; Princess Margaret Hospital, University Health Network; University of Toronto, Toronto, ON, Canada; Sunnybrook Health Sciences, Toronto, ON, Canada.

Background: There are no serum biomarkers for the accurate diagnosis of clear cell renal cell carcinoma (ccRCC). Diagnosisand the decision of nephrectomyrely on imaging which is not always accurate.Non-invasive diagnostic biomarkers are urgently required.

Design: In this study, we preformed quantitative proteomics analysis on a total of 199 patients including 30 matched pairs of normal kidney and ccRCCusing isobaric tags for relative and absolute quantitation (iTRAQ) labeling and LC-MS/MS analysis to identify differentially expressed proteins.

Results: We found 55 proteins that were significantly dysregulated in ccRCC compared to normal kidney tissue. Of these, 54 were previously reported to play a role in carcinogenesis, and 39 are secreted proteins. Dysregulation of alpha-enolase (ENO1), L-lactate dehydrogenase A chain(LDHA), heat shock protein beta-1(HSPB1/ Hsp27), and $10 \mathrm{kDa}$ heat shock protein, mitochondrial(HSPE1) was confirmed in two independent sets of patients using western blot and immunohistochemistry. Pathway analysis showed that glucose metabolism is altered in ccRCC compared to normal kidney tissue. In addition, we examined the utility of $\mathrm{Hsp} 27$ as biomarker in the serum and urine. Hsp27 had significantly higher expression in the urine of ccRCC patients $(\mathrm{p}<0.05)$ compared to normal individuals. High serum Hsp27 was also associated with ccRCC $(\mathrm{p}=0.008)$ compared to normal and with high grade (Grade 3-4) when compared to low grade (grade 1-2) tumors $(\mathrm{p}=0.013)$.

Conclusions: These data together identified potential diagnostic biomarkers forccRCC and shed new light on the molecular mechanisms that are dysregulated and contribute to the pathogenesis of ccRCC. Hsp27 is a promising diagnostic marker for ccRCC patients. Also, molecular profiling can help pave the road to the discovery of new therapies that improve patient survival.

\section{Smooth Muscle Tumors of the Scrotum: A Comparative Histologic} Study of 18 Cases

A Matoso, S Chen, JA Plaza, JI Epstein. Johns Hopkins Hospital, Baltimore, MD; Rhode Island Hospital/Brown University, Providence, RI; Medical College of Wisconsin, Milwaukee, WI.

Background: Smooth muscle tumors of the scrotum are very uncommon and those with degenerative-appearing atypia, variably designated as "atypical", "symplastic", or "bizarre" leiomyomas are extremely rare with only 11 cases in the literature. Given their rarity, diagnostic criteria and the prognosis of symplastic leiomyomas are not well established.

Design: We describe 9 cases of symplastic leiomyomas of the scrotum, and compare their histopathologic characteristics to 8 usual leiomyomas and 1 leiomyosarcoma (LS) of the scrotum. All symplastic leiomyomas and the LS were consults to the senior author. Results: The preoperative diagnosis was scrotal tumor or cyst in all cases. The mean age was 40 years (32-59) for usual and 60 (37-79) for symplastic leiomyomas. The patient with LS was 49 . Submitting diagnoses for symplastic leiomyomas were: atypical spindle cell lesion $(n=3)$; probably LS $(n=1)$; LS $(n=1)$; and none given $(n=4)$. Symplastic leiomyomas were diagnosed when there was moderate-severe cytological atypia, yet was degenerative-appearing with multinucleation or smudged chromatin in the setting of low N/C ratio, low cellularity, and virtually no mitotic activity. The mean size was $1.0 \mathrm{~cm}$ and $0.8 \mathrm{~cm}$ for usual and symplastic leiomyomas, respectively. The LS measured $0.8 \mathrm{~cm}$ and had high N/C ratio, high cellularity, nuclear pleomorphism and 
hyperchromasia. 5/8 usual and 4/9 symplastic leiomyomas showed at least one ill-defined border simulating infiltrative growth. Resection margins were positive in $4 / 8$ usual and $3 / 9$ symplastic leiomyomas, and in the LS. Ki67 labeling in usual leiomyomas was on average $2.5 \%(1-5)$ and $4.6 \%(2-10)$ in symplastic leiomyomas. Mitoses were absent in all cases of usual and symplastic leiomyomas, while it reached 7 per HPF in the LS. None of the symplastic leiomyomas recurred after a median follow-up of 30 months. The patient with LS had no metastasis at presentation with no additional follow-up. Conclusions: Clinical presentation and size of the lesion was similar for all groups. Scrotal symplastic leiomyomas may have an ill-defined infiltrative border, mimicking malignancy and resulting in a positive margin, yet is no different than usual leiomyomas. Ki67 was low in symplastic leiomyomas which along with their favorable follow-up and experience in other organs justifies a benign diagnosis. High cellularity and high mitotic activity are the most reliable features for the diagnosis of scrotal LS.

\section{Squamous Cell Carcinoma (SCC) of the Scrotum: A Series of 26 Cases}

A Matoso, $H$ Ross, J Allbritton, JI Epstein. Johns Hopkins Hospital, Baltimore, MD; Miraca Life Sciences, Lutherville-Timonium, MD.

Background: SCC of the scrotum is a rare neoplasm and the first malignancy linked to occupational exposure, initially known as chimney sweepers' cancer. The current epidemiology and clinicopathologic features are largely unknown due to its low incidence.

Design: We describe the histopathologic features of 26 patients with diagnosis of SCC of the scrotum from 10 institutions. 9 cases were consults.

Results: The mean age at presentation was 56 years (range: $39-74)$. White to black ratio was 2.3:1. There was no predominant occupation with the majority being white-collar professionals. 2 patients worked in building maintenance, 1 in car repair and 1 was a veteran. Clinical history of genital warts or condylomas was present in 5 patients, and 7 patients had history of multiple skin cancers including melanoma, basal cell carcinoma and other SCCs. One patient had invasive SCC of the anus and one had SCC in-situ of the penis. Other comorbidities included 2 patients with HIV, one patient had hidradenitis, one had chronic history of scrotal infections with abscess, one case appeared to arise in an epidermal inclusion cyst and one patient had history of lichen planus. One patient had long history of tanning bed use. Morphologically, the majority was usual type ( $\mathrm{n}=17)$ followed by basaloid $(\mathrm{n}=5)$ and warty $(\mathrm{n}=4) .18$ cases were in-situ and 8 were invasive $(31 \%)$. P16 was strongly and diffusely positive in $8(30 \%)$ cases; HPV high risk was confirmed in 4 cases and HPV 6/11 in one case. The average Ki67 was $40 \%$ for the basaloid type, $27 \%$ for the usual type and $18 \%$ for the warty type. Strong and diffuse positive 533 was seen in only 1 case (also 16 positive). All patients were treated with local excision only, and $11(42 \%)$ had positive margins after first excision, requiring re-excision. 3 patients were treated with imiquimod after local excision. The median follow-up was 30 months (available in 20). 3 patients recurred and were treated with re-excision; one patient received radiotherapy. None of the patients had metastases or died of the disease.

Conclusions: SCC of the scrotum in the U.S. currently affects primarily white-collar professionals. The majority present with in-situ lesions and the high rate of positive margins at first excision suggest that they are clinically ill-defined lesions. Positive p16 and HPV in-situ hybridization indicate that a proportion of cases are associated with HPV infection. Other apparent risk factors include HIV infection, chronic scrotal inflammatory conditions, and possibly history of tanning bed use.

\section{Molecular Analysis of Inverted Urothlelial Papillomas Reveals} Frequent HRAS Q61R Mutations and Confirms Lack of Association with HPV Infection

A McDaniel, Y Zhai, K Cho, J Montgomery, G Palapattu, J Siddiqui, T Morgan, A Alva, A Weizer, C Lee, A Chinnaiyan, S Tomlins, R Mehra. University of Michigan, Ann Arbor, MI

Background: Inverted urothelial papilloma (IUP) is an uncommon neoplasm with distinct morphologic features. Studies regarding the role of human papillomavirus (HPV) in the etiology of IUP have provided conflicting data, with some indicating a high incidence of HPV infection and others the opposite. Additionally, little is known regarding the molecular alterations present in IUP. Here, we evaluated for the presence of HPV and for other mutations at genomic "hot spot" regions for human cancer within a cohort of IUP and other inverted urothelial neoplasms.

Design: A cohort of 7 IUP along with 5 inverted low-grade urothelial carcinomas (UCA) and 5 inverted high-grade UCA were included. All diagnoses were confirmed according to current WHO criteria. Immunohistochemistry (IHC) for p16 and in situ hybridization (ISH) for HPV 16 and 18 were performed on all cases. DNA was extracted from formalin-fixed, paraffin-embedded (FFPE) sections and PCR was performed using the GP5/6 and My09/11 consensus primer sets to detect and type HPV on all cases. Additional DNA from 5 IUP and all inverted UCA cases was subjected to multiplex PCR to generate libraries of 207 amplicons from frequently mutated genomic regions

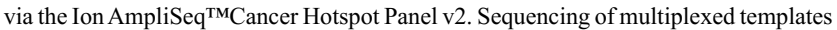
was performed using the IonTorrent Personal Genome Machine. Sequence analysis was performed in Torrent Suite 3.6, with alignment by TMAP and variant calling using the Torrent Variant Caller plugin.

Results: HPV was not detected in any case of IUP or inverted UCA by either ISH or by PCR. p16 expression was less frequent in IUP compared to inverted UCA, but was inconsistent across all groups. Sequencing data showed recurrent mutations in HRAS (Q61R) in 3 of 5 IUP, and one IUP with a mutation in FGFR3 (S249C). Interestingly, one case of inverted low grade UCA with morphology notable for IUP-like growth in some areas also carried a HRAS Q61R mutation. Additional mutations of Ras pathway members were detected including KRAS G12R in one case of inverted low grade UCA and BRAF G649A in one case of inverted high grade UCA.
Conclusions: We describe for the first time the presence of HRAS Q61R mutations at a high frequency in IUP. The presence of other Ras pathway member mutations at a rate higher than expected for typical UCA indicates this pathway may contribute to pathogenesis of inverted urothelial neoplasms. Additionally, we did not find evidence supporting a role for HPV in the etiology of IUP.

1021 Pathology Reporting v. Research Review for Prostate Cancer Gleason Scoring: Insights from Tumor Mapping and Volumetric Studies $V$ Mehta, J Chang, K Matsumoto, HA Al-Ahmadie, Y-B Chen, A Gopalan, SK Tickoo, $V E$ Reuter, SW Fine. Memorial Sloan-Kettering Cancer Center, New York, NY.

Background: Prior literature has compared pathology reports for prostate cancer with centralized review by experts, often finding significant discrepancy in Gleason score (GS). Some of these differences may be explained by the evolution in the application of Gleason grading as well as when and how to assign tertiary patterns. Research-level review with tumor mapping and volumetric studies of Gleason patterns has been seldom reported or compared with clinical reports. Using a large cohort of GS 6-7 cases, we compared our clinical diagnoses with the results of tumor volume studies, specifically focused on percentage of Gleason pattern (GP) 4, performed on the same specimens. Design: Tumor maps with delineation of GP 3 and 4 were created for 1620 wholemounted and entirely-submitted radical prostatectomy specimens with GS 6-7, diagnosed from 2006 to 2012 . $288(18 \%)$ were reported as GS $3+3=6,90(5 \%)$ as $3+3=6$ with tertiary GP $4,1065(66 \%)$ as $3+4=7$ and $177(11 \%)$ as $4+3=7$. Maps were digitally scanned and volumetric studies were performed to obtain \% GP 3 and 4. Pathology-reported GS (pGS) was compared with research-reviewed GS (rGS) influenced by volumetric data.

Results: pGS 3+3=6 [n=288]: rGS - 67/288 (23\%) cases had some \% GP 4 ( mean $=0.95 \%$; median $=2.1 \%$; range $=0$ to $31 \%$ ). Of those, $\%$ GP 4 was: $<5 \%, 5-10 \%$ and $>10 \%$ in 50,11 and 6 cases, respectively.

pGS 3+3=6 with tertiarv GP 4 [n=90]: rGS - $\%$ GP 4 ranged from 0 to $18 \%$ (mean $=0.47 \%) . \%$ GP 4 was: $0 \%$ (rGS $3+3=6),<5 \%, 5-10 \%$ and $>10 \%$ in $29(32 \%)$, $52(58 \%), 6(7 \%)$ and $3(3 \%)$ cases, respectively.

pGS 3+4=7 [n=1065]: rGS - \% GP 4 ranged from 0 to $95 \%$ (mean=12\%). $\%$ GP 4 was: $0 \%(\mathrm{rGS} 3+3=6),<5 \%, 5-10 \%, 10-50 \%$ and $>50 \%(\mathrm{rGS}>3+4=7)$ in $68(6 \%)$, $376(35 \%), 225(21 \%), 343(32 \%)$ and $53(5 \%)$ cases, respectively.

pGS 4+3=7 [n=177]: rGS - $\%$ GP 4 percentage ranged from 3.3 to $100 \%$ (mean $=62 \%$ ). $\%$ GP 4 was: $<10 \%, 10-50 \%(\mathrm{rGS}<4+3=7)$ and $>50 \%$ in $2(1 \%), 57(32 \%)$ and 118 $(67 \%)$ cases, respectively.

Conclusions: Research review with volumetric studies reveals variability in assigning Gleason pattern 4 in cases with GS 6 to 7 . Specifically, the data highlights interpretive differences in cases with $<5 \%$ GP 4 . Possible explanations include: 1) varying interpretation of particular morphologies and their impact on GS assignment over time (mucin/mucinous fibroplasia, small glomerulations, poorly formed glands [especially relevant to pGS $3+3=6$ and 6 with tertiary GP 4], intraductal carcinoma [especially relevant to pGS 7]);2) assignment of GS to dominant nodule v. overall GS [especially relevant to $\mathrm{pGS} 7]$.

\section{The Stimulating Effect of Interleukin-6 in TMPRSS2-ERG Positive} Prostate Cancer

$C$ Merz, M Nowak, $W$ Vogel, MA Svensson, J Carlsson, O Andren, $N$ Wernert, $G$ Kristiansen, S Perner. Department of Prostate Cancer Research, Bonn, NordrheinWestfalen, Germany; University Hospital of Bonn, Bonn, Nordrhein-Westfalen, Germany; University Hospital of Örebro, Örebro, Sweden.

Background: Prostate cancer is the most frequently diagnosed cancer in men in the western world. About $50 \%$ of all cases are associated with the TMPRSS2-ERG gene fusion resulting in an overexpression of the ERG transcription factor. The clinical significance of the gene fusion remains still unclear. The pleiotropic cytokine interleukin-6 (IL-6) however is frequently overexpressed with advanced and metastatic prostate cancer. In this study we addressed the role of ERG in IL-6 production. Moreover, we aimed at understanding interactions between signaling pathways downstream of the IL-6 receptor (CD126) and the androgen receptor (AR).

Design: Using prostatectomy in a tissue microarray format of 116 patients $(n=90$, prostate cancer; $n=26$, benign prostate tissue) from Cohort of Swedish Men we analyzed the co-expression of ERG and IL-6 using immunohistochemical double staining.

Results: Expression of ERG is strongly correlated with IL-6 expression $\left(r^{2}=0.7656 ; p<\right.$ 0.0001). Forced expression of ERG in the prostate tumor cell lines PC-3 and LNCaP resulted in significantly increased secretion of IL-6. Antibody-mediated blockade of CD126 in PC-3 cells significantly reduced the production of IL-6, suggesting a feedforward mechanism driving the IL-6 production. Treatment with dihydrotestosterone (DHT) in hormone-sensitive LNCaP cells increased the secretion of IL-6, conversely treatment with IL-6 exhibited a translocation of the AR to the nucleus. These data point to a cross-talk between the signaling pathways elicited by IL-6 and DHT.

Conclusions: In summary, we determined the strong co-expression of ERG and IL-6 in a large patient cohort and demonstrated at which point the IL-6 and AR receptor pathway interact.

1023 Heterogeneous Clinicopathologic Features of Intraductal Carcinoma of the Prostate (IDC-P): A Comparison between "PrecursorLike" and Regular Type Lesions

KMiyai, MK Divatia, SS Shen, AG Ayala, JY Ro. Houston Methodist Hospital, Houston, TX; Weill Cornell Medical College of Cornell University, New York, NY. Background: Intraductal carcinoma of the prostate (IDC-P) has been currently described as a lesion associated with aggressive disease and considered as intraductal spread of invasive carcinoma, rather than a precursor condition. Although it is rare, we 
have found several cases of IDC-P without identifiable invasive cancer and cases with predominant IDC-P component showing a morphological spectrum from high grade prostatic intraepithelial neoplasia (HPIN) to typical IDC-P, indicating that IDC-P may not always represent intraductal spread of pre-existing invasive cancer. We aimed to compare the clinicopathologic features between the morphologically "regular type" and "precursor-like" IDC-Ps.

Design: We evaluated 901 prostate cancer patients treated with radical prostatectomy between 2006 and 2012 in our hospital and reviewed all whole-mount slides. We defined precursor-like IDC-P cases as 1) cases of IDC-P without coexisting identifiable invasive adenocarcinoma or 2 ) cases of predominant ( $>80 \%$ of total tumor volume) IDC-P which showed clear morphological transition from HPIN to IDC-P. Data on age, Gleason score (GS, $\geq 8$ or not), surgical margin, pT stage (pT2 or more), extraprostatic extension (EPE), lymphovascular invasion, seminal vesicle (SV) invasion, and lymph node metastasis were analyzed.

Results: Among 901 patients, 141 (15.7\%) regular type IDC-P cases and $14(1.6 \%)$ precursor-like IDC-P cases ( 3 cases of IDC-P without coexisting invasive cancer; 11 cases of predominant IDC-P) were identified. In the former 3 cases, invasive carcinoma components which were distant from IDC-P were identified. The median age of the patients was 63 years in both subsets. Regular type IDC-P cases showed higher GS $(P=0.027)$, more frequent EPE $(P=0.040)$ and $S V$ invasion $(P=0.010)$, and more advanced $\mathrm{pT}$ stage $(\mathrm{P}=0.00073)$ than precursor-like IDC-P cases.

Conclusions: Our data suggest that IDC-P may be heterogenous with variable clinicopathologic features. Although further molecular genetic studies are needed to confirm this theory, we suggest that IDC-P does not always represent intraductal spread of pre-existing invasive cancer and have aggressive behavior; at least a subset of IDC-P may be a precursor lesion of invasive carcinoma, akin to HPIN.

1024 Clinicopathologic Analysis of Intraductal Cribriform Lesions of Prostate: Intraductal Carcinoma of Prostate (IDC-P), High-Grade Prostatic Intraepithelial Neoplasia (HPIN), and Atypical Cribriform Lesion (ACL)

KMiyai, MK Divatia, SS Shen, AG Ayala, JY Ro. Houston Methodist Hospital, Houston, TX; Weill Cornell Medical College of Cornell University, New York, NY

Background: IDC-P has been recently shown as a predictive marker of clinically aggressive prostate cancer. While it is important to distinguish IDC-P from HPIN, both are intraductal cribriform lesions, however, it appears that they exist along a morphologic spectrum. A group of similar lesions that does not fit well with either IDC-P or HPIN are termed "atypical cribriform lesions (ACLs)". The clinicopathological significance of these ACLs has not been well elucidated. We aimed to evaluate the relationship between these three types of intraductal cribriform lesions and clinicopathologic parameters.

Design: We evaluated 901 consecutive radical prostatectomy whole mount specimens for prostate cancer operated by a single surgeon between 2006 and 2012. Presence of intraductal cribriform lesions (IDC-P, HPIN, and ACL) were recorded and correlated with patient's age, other important tumor pathologic parameters including Gleason score (GS $\geq 8$ or not), tumor volume, surgical margin (SM), pT stage (pT2 or more), extraprostatic extension (EPE), lymphovascular invasion (LVI), seminal vesicle invasion (SVI), and lymph node metastasis (LN). IDC-P was defined as follows; 1) solid or dense cribriform lesions or 2) loose cribriform or micropapillary lesions with prominent nuclear pleomorphism (nuclear size greater than $6 \mathrm{x}$ normal) $+/$-necrosis. ACL was defined as loose cribriform lesion without nuclear pleomorphism or necrosis. Results: Among 901 patients, IDC-P, ACL, and HPIN were identified in 155, 22, and 436 cases respectively. Patients with IDC-P showed higher GS, higher tumor volume, more frequent LVI, higher rate of positive SM, and more advanced stage (EPE, SVI) and more frequent nodal metastasis than those patients with $\operatorname{HPIN}(\mathrm{P}<0.0001)$. Patients with ACL also had higher GS $(\mathrm{P}=0.00016)$, higher tumor volume $(\mathrm{P}=0.025)$, and more advanced stage $(\mathrm{P}=0.023)$ than patients with HPIN. However, there was no statistically significant difference between patients with IDC-P and ACL.

Conclusions: We confirmed that the presence of IDC-P is strongly associated with aggressive clinicopathological behavior. We also showed that ACL should be distinguished from HPIN as an intermediate risk category and consideration should be given to have more active surveillance or biopsy than HPIN.

1025 Intramucosal Involvement of Seminal Vesicle by Prostate Carcinoma Is More Likely Direct Extension from Seminal Vesicle Wall Invasion Rather Than Extension from Invaginated Extraprostatic Space/ Ejaculatory Duct System

K Miyai, S Pina Oviedo, MK Divatia, SS Shen, AG Ayala, JY Ro. Houston Methodist Hospital, Houston, TX; Weill Cornell Medical College of Cornell University, New York, NY.

Background: We have shown the presence of seminal vesicle (SV) intraepithelial involvement of prostate cancer in cases with SV wall invasion of prostate cancer (Mod Pathol.26:241A, 2013). With regard to the manner of SV intramucosal involvement, there could be two putative mechanisms; direct mucosal invasion from SV muscular wall involved by prostate cancer or intramucosal involvement of cancer to SV directly from the invaginated extraprostatic space (IES)/ejaculatory duct system, akin to the phenomenon of intraductal spread of prostate carcinoma. We aimed to evaluate the relationship between SV intramucosal involvement and IES/ejaculatory duct system involvement and to clarify the manner of SV intramucosal involvement.

Design: We evaluated 30 radical prostatectomy whole mount specimens for prostate cancer with SV wall invasion (pT3b) between 2009 and 2012. SV invasion was divided into 2 types based on the absence (type 1) or presence (type 2) of intramucosal involvement besides SV muscular invasion. The presence of IES involvement and ejaculatory duct mucosal involvement of prostate cancer were also recorded and these findings were correlated with clinicopathologic data including age, Gleason score (GS, $\geq 8$ vs. $<8$ ), surgical margin, lymphovascular invasion (LVI) and lymph node metastasis
Results: Among 30 prostates with SV invasion, $22(73 \%)$ and $8(27 \%)$ cases showed types 1 and type $2 \mathrm{SV}$ involvement, respectively. IES involvement was observed in 13 cases; 9 in type 1 cases and 4 in type 2 cases. However, none of these cases showed ejaculatory duct mucosal involvement of prostate cancer. There was also no correlation between presence of IES involvement and types of seminal vesicle invasion $(\mathrm{P}=0.48)$. No significant differences were found between the types of SV invasion and other parameters such as age, Gleason score, surgical margins status, LVI and lymph node metastasis $(\mathrm{P}>0.05)$

Conclusions: We have shown that the presence of IES involvement of prostate cancer does not predict SV intramucosal involvement. Furthermore, our data indicates that SV intramucosal involvement is more likely due to direct mucosal involvement from the prostate cancer in SV wall rather than the extension of cancer from the IES/ejaculatory duct system. It appears that SV wall invasion with intramucosal involvement (type 2) does not infer a worse prognosis than SV wall invasion alone (type 1).

\section{Expression of Androgen Receptor Splice Variant AR3 in Benign} and Malignant Prostate Tissue

ZMo, JSquires, Y Qiu, J Huang. University of California, Los Angeles, CA; University of Maryland School of Medicine, Baltimore, MD.

Background: Androgen receptor (AR) signaling is required for the growth and survival of prostate cancer (PCa), and hormonal therapy is the treatment of choice for patients with advanced and metastatic PCa. Although hormonal therapy is effective initially, the disease eventually recurs as castration resistant PCa (CRPC). A novel hypothesis to explain the therapeutic failure is that hormonal therapy, while inhibiting the function of the wild-type, full-length AR, promotes the expression of AR splice variants that lack the ligand-binding domain. Without the normally inhibitory ligand binding domain, these AR variants are androgen-independent and constitutively active in the androgendeprived environment, which may contribute to the progression of the disease to the castration resistant stage. Some patients with PCa recur with small cell neuroendocrine carcinoma (SCNC) after hormonal therapy, which has become more common after therapy with newer drugs such as Zytiga and MDV3100. The mechanism is unclear and the role of AR splicing variants in SCNC has not been studied.

Design: We used a monoclonal antibody against AR3, the most common AR splice variant, to stain tissue microarrays containing paired benign prostate and hormonenaive PCa (HNPC, never treated with hormonal therapy, $\mathrm{n}=650)$, CRPC $(\mathrm{n}=30)$, and $\mathrm{SCNC}(\mathrm{n}=15)$ by immunohistochemistry. The staining was dichotomized as positive (nuclear staining) or negative (no nuclear staining). Statistical analysis was performed by Chi suqare test.

Results: All benign prostate cores were negative for AR3. AR3 staining was positive in $15 \%$ of HNPC . In contrast, there is significant increase AR3 expression in CRPC $(30 \%, \mathrm{P}=0.028)$, and none of the SCNC were positive for AR3.

\section{The expression of $\mathrm{AR} 3$ in benign and neoplastic prostate tissue}

\begin{tabular}{|c|c|c|}
\hline & AR3 positive rate & case number \\
\hline Benign & $0 \%$ & 150 \\
\hline HNPC & $15 \%$ & 650 \\
\hline CRPC & $30 \%$ & 30 \\
\hline SCNC & $0 \%$ & 15 \\
\hline
\end{tabular}

Conclusions: 1). AR3 expression is a specific marker of $\mathrm{PCa}$; 2). AR3 is expressed in a subset of HNPC, suggesting that it may contribute to the carcinogenesis in such cases, and it is important to determine if AR3 positive cases are poor responders to hormonal therapy; 3). Compared with hormone naive PCa, twice as many CRPC cases express AR3, suggesting that hormonal therapy-induced AR3 expression may be a mechanism of therapy resistance in some patients. 4). SCNC is always negative for AR3, indicating that this disease may be independent of AR or its splice variant, and probably develops through a different mechanism.

1027 Evaluation of the Mid/Hindgut Differentiation Marker SATB2 in Glandular Lesions of the Urinary Bladder

SK Mohanty, SC Smith, E Kim, F Chung, DE Hansel, MN dePeralta-Venturina, DJ Luthringer, $S$ Wang, MB Amin. Cedars-Sinai Medical Center, Los Angeles, CA; University of California, San Diego, San Diego, CA.

Background: The biologic relationship between urachal carcinomas, non-urachal adenocarcinomas, and glandular metaplastic processes of the urothelium remains obscure. The DNA binding protein, Special AT-rich sequence-binding protein 2 (SATB2) has shown promise as a marker of intestinal differentiation in anatomic structures arising from the the mid- and hindgut, with some studies finding specificity exceeding CDX2. SATB2 has not been studied in glandular lesions, neoplastic or metaplastic, of the urinary bladder, where classification of urachal vs non-urachal processes remains dependent primarily on clinical context and where a specific immunoprofile for urachal lesions would have diagnostic utility.

Design: Immunohistochemical (IHC) stain for SATB2 was applied to evaluate whole sections of non-urachal glandular lesions of the bladder, including adenomas, non-urachal adenocarcinomas, urothelial carcinomas with glandular differentiation, and cystitides cystica et glandularis, including intestinalis $(\mathrm{n}=37)$, and urachal adenocarcinomas $(\mathrm{n}=10)$. SATB2 stain was evaluated by intensity $(0-3)$ and proportion $(0,<5 \% ; 1+, 5-25 \% ; 2+, 26-50 \%$; or $3+,>50 \%)$ and compared among lesions by Fisher's Exact Test.

Results: SATB2 nuclear expression was intense and widespread in 14/14 lesions with intestinal differentiation, both neoplastic and metaplastic. Cystitides cystica et glandularis without intestinal differentiation were uniformly negative $(n=24)$, though several lesions showed foci of or individual goblet cells staining positively for SATB2 in otherwise conventional cystitides glandularis $(n=5)$. In contrast, urachal adenocarcinomas with mucinous morphology showed low intensity/patchy stain among a subset (3/9), with the remainder negative $(\mathrm{p}<0.0001)$. 
Conclusions: SATB2 expression is exquisitely sensitive for intestinal differentiation in glandular lesions of the bladder, including expression in very focal or incipient metaplastic lesions. In contrast, mucinous urachal adenocarcinomas show markedly attenuated expression of SATB2. Combinatorial testing of SATB2 with CDX2 and beta-catenin is underway, to the end of defining a useful urachal-specific immunoprofile.

1028 Biomarkers Differentially Expressed in Prostatic Adenocarcinoma Gleason Score $3+4=7$ vs Gleason Score $4+3=7$ Identified by Global Transcriptome Sequencing of Formalin-Fixed Paraffin Embedded Tissue CS Moreno, Q Long, J Xu, S Sannigrahi, BA Johnson, W Zhou, T Gillespie, JY Park, RK Nam, L Sugar, A Stanimirovic, AK Seth, JA Petros, AO Osunkoya. Emory University School of Medicine, Atlanta, GA; Veterans Affairs Medical Center, Atlanta, GA; Moffitt Cancer Center, Tampa, FL; Sunnybrook Health Sciences Centre, Toronto, Canada. Background: The Gleason grading system for prostate cancer is unique in that the final Gleason score is typically calculated by adding the most prevalent pattern (primary pattern) to the second most prevalent pattern (secondary pattern). The only exception to this rule is in needle core biopsies (when there are three patterns) in which the most prevalent pattern is added to the highest pattern to obtain the Gleason Score. It has been suggested that primary Gleason 4 pattern and Gleason 3 pattern tumors represent different disease states (i.e. $3+4=7$ vs $4+3=7$ ), and several studies have supported the concept that the primary Gleason pattern of patients with a Gleason score of 7 is predictive of outcome. We investigated the differential gene expression profile between patients stratified into these two groups.

Design: To investigate the differences in gene expression, we performed RNAseq analysis and DEseq differential gene expression analysis comparing 43 samples with Gleason $3+4=7$ (primary pattern 3 ) to 22 samples with Gleason $4+3=7$ (primary pattern 4). Samples were obtained from formalin-fixed paraffin-embedded (FFPE) radical prostatectomy specimens.

Results: The mean patient age was 61 years (range $47-74$ years). We observed several genes differentially expressed relevant to prostate cancer, including upregulated (miR10A, Twist, HOXC6, AR) and downregulated (ERG, EGF, SOX9, WIF1, WNT5A, SHH) genes in cases with Gleason score $4+3=7$ compared to Gleason score $3+4=7$. Ingenuity pathway analysis also determined that the top biological functions associated with the differentially expressed genes were abnormal bone morphology, cell differentiation, genital tumors, and prostatic bud formation.

Conclusions: We have identified genes that are differentially expressed in cases with Gleason score $4+3=7$ compared to those with Gleason score $3+4=7$. In addition, the fact that the most significant biological annotation was abnormal bone morphology suggests that higher Gleason primary pattern tumors intrinsically express genes that may facilitate metastasis to the bone. Future studies will be needed to determine the functional role of these genes associated with bone morphology in prostate cancer progression and metastasis.

1029 Global Transcriptome Sequencing of Formalin Fixed Paraffin Embedded Patient Samples Identifies Biomarkers That Are Differentially Expressed between Caucasian and African American Men with Prostate Cancer

CS Moreno, J Xu, Q Long, S Sannigrahi, BA Johnson, W Zhou, T Gillespie, JY Park, RK Nam, L Sugar, A Stanimirovic, AK Seth, JA Petros, AO Osunkoya. Emory University School of Medicine, Atlanta, GA; Veterans Affairs Medical Center, Atlanta, GA; Moffitt Cancer Center, Tampa, FL; Sunnybrook Health Sciences Centre, Toronto, Canada. Background: Prostate cancer ( $\mathrm{PCa}$ ) is responsible for one of the greatest racial health disparities, with African-American (AA) men having a $60 \%$ greater incidence rate than Caucasian (CA) men and suffering from 2.9 times greater the number of years of potential life lost before the age of 75 . The underlying etiology of these disparities has not been fully elucidated, but have persisted for decades, including in equal access-equal care settings, suggesting underlying biological differences.

Design: We performed global RNA-sequencing on formalin fixed paraffin embedded (FFPE) radical prostatectomy samples from AA and CA patients at our institutions. We performed comparative differential gene expression analysis using DESeq to identify genes differentially expressed between $\mathrm{PCa}$ in this cohort of AA and CA patients.

Results: A total of 70 patients with available tissue blocks were selected. Forty eight patients $(69 \%)$ were CA and 22 patients (31\%) were AA. Mean patient age was 61 years (range: 47-78 years). We observed a number of genes relevant to $\mathrm{PCa}$ biology including ETV5, ZEB1, ZEB2, B2M, FYN, and miR-183 that were differentially expressed between AA and CA patients by DESeq analysis. The depth of coverage obtained for mitochondrial RNA sequences was high enough (much greater than 100x) that we were able to identify recurrent single nucleotide polymorphisms (SNPs) in the mtDNA of the patient samples. Four of these SNPs (T4216C, C15452A, A14769G, and C8932T) were of particular interest. All of these SNPs result in non-synonymous amino-acid substitutions of mitochondrial protein-coding genes. The T4216C and C15452A SNPs almost always co-occur (p-value $=1.57 \mathrm{e}-13$ ), and are mutually exclusive of the strongly co-occurring A14769G and C8932T SNPs. Moreover, the T4216C/C15452A are not only more frequent in $\mathrm{PCa}$ patients, but also are more frequent in patients with $\mathrm{BCR}$, and were not observed in any AA patients. The A14769G/C8932T SNPs were more frequent in patients without $\mathrm{BCR}$ and were observed only in AA patients.

Conclusions: We have identified genes that are differentially expressed between AfricanAmerican and Caucasian PCa patients, and mitochondrial SNPs that are associated with both race and outcome. These findings suggest that there may be inherited biological mechanisms that partially account for racial disparities in PCa.
1030 GATA3 Expression in Clear Cell Papillary Renal Cell Carcinoma and Renal Cell Carcinoma with Prominent Leiomyomatous Proliferation Is a Further Evidence of the Relationship between These Two Entities

E Munari, M Brunelli, D Segala, S Gobbo, GJ Netto, L Cheng, JN Eble, B Delahunt, MG Raspollini, R Tardanico, G Martignoni. University of Verona, Verona, Italy; Pederzoli Hospital, Peschiera del Garda, Italy; Johns Hopkins University, Baltimore, MD; Indiana University, Indianapolis, IN; Wellington University, Wellington, New Zealand; University of Firenze, Firenze, Italy; University of Brescia, Brescia, Italy. Background: Among newly recognized renal neoplasms characterized by cells with clear cytoplasm, two low grade tumors named clear cell papillary renal cell carcinoma (CCPRCC) and renal cell carcinoma with prominent leiomyomatous proliferation (RCCPL) have been described. These tumors are both characterized by cytokeratin 7 (CK7) immunoexpression and show overlapping morphological features with the most common conventional clear cell renal cell carcinoma (CCRCC). GATA3, a protein essential for regulating Th2 development and function, is expressed during human kidney embryogenesis and is necessary for nephric duct morphogenesis in the pro/ mesonephric kidney. A few data regarding the expression of GATA 3 in the different morphotypes of renal cell carcinoma are available. In this study we aimed at evaluating the immunoexpression of GATA3 in a set of CCPRCC and RCCPL cases and in a large consecutive series of CCRCC.

Design: Archival formalin fixed paraffin embedded (FFPE) tissue from 13 CCPRCC and 6 RCCPL cases were selected. FFPE slides were stained for GATA3 (clone L50-823, BD Pharmingen, San Diego, CA). Expression status of GATA3 was evaluated also in a series of 180 CCRCC using whole sections. Western-Blot (WB) was used to confirm the expression of GATA3 in CCPRCC and RCCPL.

Results: GATA3 expression was present in 7/13 of CCPRCC and 3/6 RCCPL cases that we studied with a mean of $40 \%$ of positive neoplastic cells for both type of tumor. All CCRCC cases showed complete negativity for GATA3. WB analysis confirmed GATA3 expression in cases that stained positive for the marker.

Conclusions: 1) GATA3 expression in clear cell papillary renal cell carcinoma and renal cell carcinoma with prominent leiomyomatous proliferation is a further evidence of the relationship between these two entities and an additional element of distinction from conventional clear cell renal cell carcinoma. 2) GATA3 can be a useful marker in the differential diagnosis with conventional clear cell renal cell carcinoma, especially in those cases that show overlapping morphological and immunohistochemical features like CK7 expression.

1031 Clear Cell Papillary Renal Cell Carcinoma: microRNA Expression Profiling and Comparison with Clear Cell Renal Cell Carcinoma and Papillary Renal Cell Carcinoma

E Munari, L Marchionni, A Chitre, M Hayashi, G Martignoni, M Brunelli, S Gobbo, P Argani, M Allaf, MO Hoque, GJ Netto. Johns Hopkins University, Baltimore, MD; University of Verona, Verona, Italy.

Background: Clear cell papillary renal cell carcinoma (CCPRCC) is a low grade renal neoplasm with morphological characteristics mimicking both clear cell renal cell carcinoma (CCRCC) and papillary renal cell carcinoma (PRCC). However, despite some overlapping features, their morphologic, immunohistochemical and molecular profiles are distinct. MicroRNAs (miRNAs) are small non-coding RNAs that play a crucial role in regulating gene expression and are involved in various biological processes, including cancer development. In order to better understand the biology of this tumor, we aimed to analyze the miRNA expression profile of a set of CCPRCC using microarray and quantitative RT-PCR.

Design: A total of 15 cases diagnosed as CCPRCC were used in this study. Among the most differentially expressed miRNA in CCPRCC, we found miR-210, miR-122, miR-34a, miR-21, miR-34b* and miR-489 to be upregulated while miR-4284, miR1202, miR-135a, miR-1973 and miR-204 were downregulated compared to normal renal parenchyma. In order to identify consensus of differentially regulated miRNA between CCPRCC, CCRCC and PRCC, we additionally determined differential miRNA expression using two publically available microarray datasets from the NCBI Gene Expression Omnibus (GEO) database (GSE41282 and GSE3798).

Results: This comparison revealed that the miRNA expression profile of CCPRCC shows some overlapping characteristics between CCRCC and PRCC. Moreover, CCPRCC lacks dysregulation of important miRNAs typically associated with aggressive behavior.

Conclusions: In summary, we describe the miRNA expression profile of a relatively infrequent type of renal cancer. Our results may help in understanding the molecular underpinning of this newly recognized entity.

1032 Development of a Reproducible Grading System for Estimating Residual Disease and Pathologic Responses to Neoadjuvant Treatment in Prostate Cancer

C Murphy, F Vakar-Lopez, L True, J Xia, R Gulati, B Montgomery, $M$ Tretiakova. University of Washington, Seattle, WA; Fred Hutchinson Cancer Research Center, Seattle, WA.

Background: Evidence-based pathologic variables that correlate with response to neoadjuvant therapy are well characterized for breast cancer, but not for prostate cancer (PC). This study evaluates interobserver agreement (IA) in characterizing the pathologic response and residual cancer burden in neoadjuvant treated prostatectomies from multiple clinical trials to identify the most reproducible variables for further correlation with treatment resistance and patient outcome.

Design: H\&E sections from 37 patients treated with combinations of lupron, dutasteride, casodex, ketoconazole, estrogen, abiraterone and enzalutamide, and 5 untreated patients were evaluated. IA between 4 pathologists was assessed for max tumor area, 
tumor volume by point-counting, tumor cellularity - \% area comprised of tumor cells, histopathologic grouping (ABC) of tumor response (PMID: 19853370), and 11 characteristics of therapy-induced changes (PMID: 17128417). Qualitative parameters were analyzed as absent, focal, and diffuse. The intra-class correlation coefficient and Cohen's kappa were used to measure IA for the continuous and qualitative tumor characteristics. Leave-one-out cross-validation was used to correlate with years of experience and identify outlier pathologist(s).

Results: IA was excellent for tumor area, tumor cellularity, and volume estimation $(\kappa=$ $0.90-0.94)$ across all treatment groups. IA for the 11 morphologic parameters was poor ( $\kappa=0.02-0.55$, see graph). IA for ABC grouping was modest $(\kappa=0.55)$. Agreement statistics did not correlate with years of experience $(\rho=0.19)$, and no pathologist was an outlier (range across change in agreement statistics $0.20-0.31$ ).

Conclusions: Previously validated tumor characteristics - tumor area, cellularity, and volume - appear to be highly reproducible measurements in PC. Poor IA for qualitative histologic characteristics of PC may be due to variable interpretation of the categories, thus suggesting a need for consensus guidelines when assessing these characteristics.

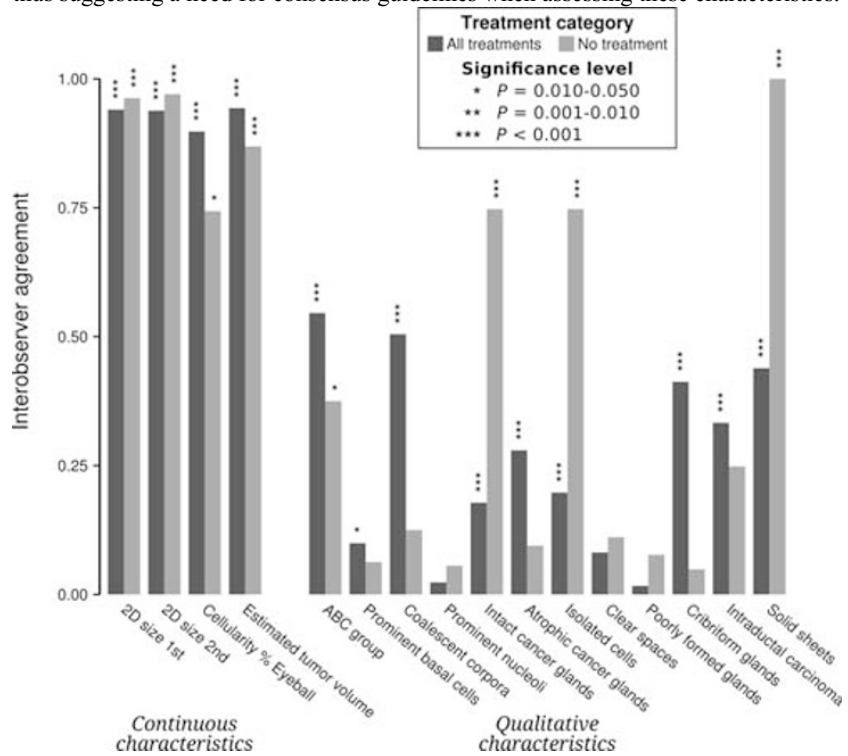

1033 Rhabdoid RCC Is Molecularly Distinct from Fuhrman Grade 4 RCC P Murugan, S-Y Yoo, T Majewski, L Patel, H Voicu, K Wani, P Tamboli, K Baggerly, B Czerniak, $K$ Aldape, $K$ Sircar. UT MD Anderson Cancer Center, Houston, TX; Baylor College of Medicine, Houston, TX.

Background: Rhabdoid differentiation is a histologic feature of of renal cell carcinomas (RCC) that is associated with a very aggressive clinical course and that is categorized as Fuhrman grade 4 . However, it is poorly understood and without adequate molecular characterization. Thus, we sought to study rhabdoid transformation in clear cell RCC at the genome wide transcript level using next generation sequencing and microarray based hybridization approaches. Specifically, our aims were to: a) study the contrast between the paired epithelioid and rhabdoid components of rhabdoid RCC; and b) examine the contrast between rhabdoid RCC and Fuhrman grade 4 RCC lacking rhabdoid/sarcomatoid areas.

Design: The rhabdoid (R) and epithelioid (E) areas were macrodissected from FFPE tissues belonging to 8 patients with rhabdoid clear cell RCC (stage 4, n=5; stage 3, $\mathrm{n}=3$ ). The transcriptome was assessed by RNA-seq (Illumina HiSeq) on 4 R/E pairs and by gene expression microarray (Illumina HumanRef-8) on another 4 R/E pairs. As controls, we used Fuhrman grade 4 clear cell RCC without any rhabdoid or sarcomatoid areas: 6 patients (stage $4, n=5$; stage $3, n=1$ ) were assessed by RNA-seq and 8 patients (stage $4, n=6$; stage $3, n=2$ ) by gene expression microarray.

Results: Bioinformatics analysis of RNA-seq data did not show any spontaneous clustering among the epithelioid (E) and rhabdoid (R) components of rhabdoid RCC with respect to genome wide expression or expression of prognostic genes $(\mathrm{ccA} / \mathrm{ccB})$. When comparing rhabdoid RCC (E/R) to non-rhabdoid Fuhrman grade 4 RCC, however, we saw many genes that were differentially expressed $(480$ genes, FDR $<0.05)$. No chimeric or gene-fusion transcripts were identified in E or R samples. Expression microarray data on independent samples validated the sequencing results in that the $\mathrm{E}$ versus $\mathrm{R}$ contrast was not significant compared to that between rhabdoid RCC (E/R) and Fuhrman grade 4 RCC (945 genes, FDR < 0.05).

Conclusions: Rhabdoid RCC is transcriptomically distinct from Fuhrman grade 4 RCC without rhabdoid or sarcomatoid features. Taken together with its more aggressive clinical behavior, our results suggest that rhabdoid RCC should be classified separately from other Fuhrman grade 4 RCC. The gene expression program of the putatively dedifferentiated rhabdoid phenotype appears to be ingrained within its better differentiated epithelioid component with no changes in expression of prognostic genes and no expression of chimeric transcripts that would molecularly stratify the two components.
1034 Type 1 Papillary Renal Cell Carcinoma: A Contemporary Study Addressing Classification, Clinicopathologic Features and Treatment Outcome

P Murugan, Y Chen, HA Al-Ahmadie, A Gopalan, SW Fine, A Gandhi, SK Tickoo, VE Reuter. Memorial Sloan-Kettering Cancer Center, New York, NY.

Background: Papillary renal cell carcinomas (PRCCs) may have variable histologies. Although controversial, some authors have suggested dividing them into type 1 and type 2 categories. In this study we aim to investigate the clinicopathological features, including disease free and overall survival, of PRCCs with any type 1 components.

Design: Eighty one cases of PRCCs with type 1 features, as described in WHO classification, diagnosed at our institution from 1995 to 2003, were reviewed. Cases with type 2 features were included as long as any clearly identifiable type 1 areas were present in the tumor. The type 2 areas were quantified in tumors with mixed histology. Multiple gross and microscopic features, including tumor size, pT stage (AJCC 2010), capsular invasion, necrosis, Fuhrman nuclear grade, nucleolar grade and mitotic rate were evaluated. Clinical features and follow up information were obtained from an IRB approved annotated clinical database. Chi-square tests were performed to evaluate the significance of the pathological parameters in relation to the outcome.

Results: The male to female ratio was 4:1. The mean age of presentation was 62 (range: 34-84) years. Tumor sizes ranged from 1.2-15 (median 3.5) $\mathrm{cm}$. Overall, there were 70 pT 1,6 pT2 and 5 pT 3 cases. 18 cases $(22 \%)$ were classified as having mixed histology, of which 4 had $>50 \%$ of type 2 areas. At median follow up of 121 (range 1-214) months, 58 patients had no evidence of disease, 7 died of other malignancies while 13 died of unknown causes. No recurrences were reported. Three patients died of disease (Table 1). Compared to $2 \%$ of cases in the remaining cohort, two of these three patients had a mitotic count of $>4 / 10$ HPF. Tumor size was the only major feature that was significantly associated with overall survival $\left(\mathrm{X}^{2}=9.27, \mathrm{P}=0.02\right)$. No significant association was noted with pT stage $(P=0.28)$, nuclear grade $(P=0.49)$, nucleolar grade $(P=0.73)$, invasion of pseudocapsule $(\mathrm{P}=0.38)$, tumor necrosis $(\mathrm{P}=0.07)$, presence of type 2 areas $(\mathrm{P}=0.64)$ or its quantity $(\mathrm{P}=0.73)$.

\begin{tabular}{|c|c|c|c|c|c|c|c|}
\hline $\begin{array}{l}\text { Case } \\
\text { No. }\end{array}$ & Type & $\begin{array}{l}\mathrm{PT} \\
(2010)\end{array}$ & $\begin{array}{l}\text { Size } \\
(\mathrm{cm})\end{array}$ & $\begin{array}{l}\text { Necrosis } \\
(\%)\end{array}$ & $\begin{array}{l}\text { Nuclear/Nucleolar } \\
\text { grade }\end{array}$ & $\begin{array}{l}\text { Mitoses/10 } \\
\text { HPF }\end{array}$ & $\begin{array}{l}\text { Follow up } \\
\text { (months) }\end{array}$ \\
\hline 1 & 1 & $1 \mathrm{~A}$ & 3.5 & 30 & $1 / 1$ & 7 & 26 \\
\hline 2 & $\begin{array}{l}\text { Mixed } \\
(40 \% \\
\mathrm{T} 2) \\
\end{array}$ & $2 \mathrm{~A}$ & 8.5 & 20 & $2 / 3$ & 0 & 49 \\
\hline 3 & $\begin{array}{l}\text { Mixed } \\
(5 \% \mathrm{~T} 2)\end{array}$ & $1 \mathrm{~A}(\mathrm{~N} 1)$ & 3.5 & 30 & $4 / 4$ & 24 & 3 \\
\hline
\end{tabular}

Conclusions: Our data indicates that PRCCs with any type 1 morphology, regardless of the presence of type 2 features have an excellent long term disease free and overall survival, independent of other features such as nuclear and nucleolar grade, stage and necrosis.

1035 Tumor Volume as a Predictor of Biochemical Recurrence in pT3 Prostate Cancer Following Robotic-Assisted Laparoscopic Prostatectomy (RALP)

E Negron, JA Cohn, $R$ Ledezma, P Dangle, SE Eggener, GP Paner, GP Zagaja. University of Chicago, Chicago, IL.

Background: Biochemical recurrence (BCR) has been reported to occur in up to $30 \%$ of patients after 5 yrs following RALP. Our objective is to determine the impact of tumor volume with the incidence of BCR in patients with non-organ confined disease with undetectable PSA after 12 months of follow up.

Design: We identified 3,063 patients who underwent RALP from March 2003 to January 2012. Follow up has been maintained in a database every 6 months for 2 years, and yearly thereafter. Patients with pathologically confirmed pT3 disease who had a postoperative undetectable PSA, who did not receive adjuvant therapy, with at least 12 months of follow-up, and who received the standard bilateral PLND were included. Tumor volume was calculated using the $\%$ of tumor involvement, multiplied by the prostatic volume, which in turn was calculated using the ellipsoid formula. Kaplan-Meier analysis was used to estimate BCR freedom probability and multivariate logistic Cox hazard regression models were used to assess predictors of BCR. Predictive accuracy of the models was quantified with Harrell's concordance index.

Results: 248 patients met the inclusion criteria. Median time to follow up was 25 months (IQR 14.6-38). Mean age at diagnosis was 61.5 years. $96.77 \%$ of patients had a GS $\geq 7$ (240). 202 patients were pT3a (81.5\%) and 42 were pT3b (18.5\%). 21 patients $(8.5 \%)$ had positive lymph nodes and 227 did not $(91.5 \%) .75$ patients $(30.2 \%)$ had positive surgical margins. BCR was observed in 49 patients $(19.8 \%)$ with a median time for recurrence of 17.03 months (IQR 12.57-25.03). Mean tumor volume was $13.7 \mathrm{ml}$. Positive node status $(\mathrm{p}=<0.01)$, tumor volume $(\mathrm{p}=<0.01)$ and Gleason $8(\mathrm{p}=0.05)$ were independently associated with the risk of having BCR in our multivariate analysis model. Having a tumor volume $>5 \mathrm{ml}$ showed a higher risk of BCR $(\mathrm{p}=0.02)$. The predictive accuracy of positive nodes was significantly improved by tumor volume, when tumor volume was considered (Harrell index; 59\% vs. 65.1\%). 


\section{KM estimated BCR freedom in PT3 patients by tumor volume}

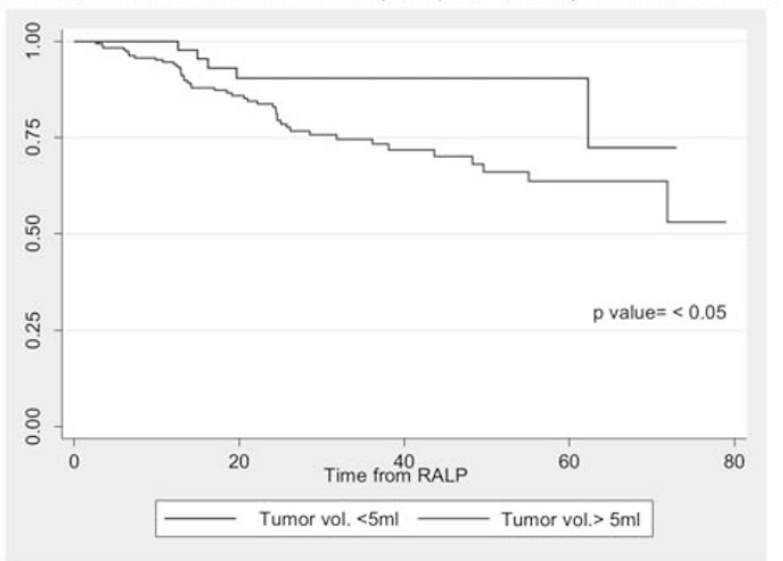

Conclusions: In patients with non-organ confined prostate cancer with undetectable PSA in postoperative period, the node positive status and tumor volume has shown to be significant predictors of BCR. Gleason 8 has also shown statistical significance as a $\mathrm{BCR}$ predictor in this cohort of patients.

\section{Low ARID1A Immunoexpression in Penile Squamous Cell} Carcinoma

GJ Netto, SM Bezerra, N Gonzalez-Roibon, A Chaux, E Munari, M Ball, I-M Shih, AL Burnett, SF Faraj. Johns Hopkins Medical Institutions, Baltimore, MD; Universidad del Norte, Asunción, Paraguay.

Background: AT-rich interactive domain 1A (ARID1A) is a tumor suppressor gene that has been described to be inactivated in several neoplasms, including epithelial ovarian and endometrial carcinomas and has been correlated with prognosis. Recently, we found a significant trend of association between low ARID1A expression and lower histologic grades in penile squamous cell carcinoma (SCC) in a cohort from Paraguay. The current study assesses ARID1A expression and its association with outcome in penile SSC from Johns Hopkins Hospital.

Design: Two tissue microarrays were constructed from 59 penile SCC at Johns Hopkins Hospital (1985 to 2013). Immunohistochemistry for ARID1A was performed (HPA005456, Sigma-Aldrich, St Louis, MO) and nuclear expression was evaluated. An $\mathrm{H}$-score was calculated as the sum of the products of intensity (0-3) multiplied by extent of expression (0 to $100 \%)$. Average $\mathrm{H}$-score per case was used for statistical analysis. Results: Median H-score was 226.7 (range: 53-284.5). A low ARID1 A expression was seen is $74.1 \%$ ( 43 of 58 ) of penile SCC. There was a significant difference between low ARID1A expression in penile $\mathrm{SCC}$ and paired benign squamous epithelium $(\mathrm{p}=0.04)$. ARID1A expression was not associated with any clinicopathologic parameter such as race $(\mathrm{p}=0.67)$, grade $(\mathrm{p}=0.08)$, chemotherapy $(\mathrm{p}=0.66)$, radiotherapy $(\mathrm{p}=1), \mathrm{pT}$ category $(\mathrm{p}=0.55)$, lymph node metastasis $(\mathrm{p}=0.49)$, visceral metastasis $(\mathrm{p}=1)$, histologic type $(\mathrm{p}=0.87)$, location $(\mathrm{p}=0.96)$, thickness $(0.097)$, lymphovascular invasion $(\mathrm{p}=0.73)$, perineural invasion $(\mathrm{p}=0.47)$. There was no correlation between low ARID1 A expression and tumor progression-free survival (HR: $1.10, \mathrm{p}=0.86$ ), overall survival (HR: 0.52, $\mathrm{p}=0.29$ ) or disease-specific survival (HR: $1.69, \mathrm{p}=0.55$ ).

Conclusions: We found a high rate of low ARID1A expression in penile SCC. In contrast to our previous study, we did not find ARID1A expression to be associated with any of the clinicopathologic parameters evaluated. In addition, we found no correlation between ARID1A expression and outcome.

\section{Keeping an Open Mind: Anorectal Disease in Prostate Biopsies} and Prostate Cancer in Rectal Biopsies

J Niakan, D Beyda, P Unger, G-O Xiao. Mount Sinai Medical Center, New York, NY; SUNY Downstate at LICH, Brooklyn, NY.

Background: Transrectal prostatic core needle biopsies pick up anorectal tissue in up to $15 \%$ of cases. While the anorectal tissue is not the focus of the procedure and may easily be overlooked, this incidental sampling may bear significant pathologic findings. On the other hand, clinically unrecognized advanced prostate cancer may extend to the anorectum and present as an anorectal lesion mimicking a primary anorectal malignancy, particularly in a limited biopsy material.

Design: A search of the Mount Sinai Medical Center pathology database was conducted for transrectal prostatic core biopsies, as well as anorectal biopsies, performed between January 1994 and August 2013. Incidental significant anorectal pathologic diseases found in prostate needle biopsies and prostate cancer identified in anorectal biopsies were included. Demographic and clinical information was also collected from these patients. Results: The search yielded over 7,000 prostate core needle biopsies performed over the past seven years with significant anorectal pathologic findings identified in nine cases. These cases included: one case of radiation proctitis (with paneth cell metaplasia from a patient with external beam radiation treatment history), five cases of active proctitis (one with a clinical diagnosis of ulcerative colitis), one rectal tubular adenoma, one anal squamous dysplasia and one anal intraepithelial neoplasia 3 (AIN 3). Prostatic adenocarcinoma was reported in six of the cases with Gleason scores of 6 to 7 . Over 20,000 rectal biopsies were searched and yielded six cases of prostatic adenocarcinoma. The clinical information provided included rectal mass, rectal mass with obstruction, anal mass with bleeding and anal stricture with bleeding. When available, the cases were reported as poorly differentiated and the diagnosis was supported by imunohistochemistry in three of the cases.

Conclusions: Although quite rare, significant anorectal pathologic diseases may be incidentally found in routine prostate biopsies. To our knowledge, this is the first reporting of anal squamous lesions presenting in prostate biopsies. This study highlights the importance of analyzing the anorectal tissue and identifying and reporting the pathologic findings in order to help direct appropriate and timely patient management. Additionally, the findings also demonstrated that advanced prostate carcinoma may present as an anorectal lesion and should be included in the differential diagnosis.

1038 The Utility of CDX2, GATA-3 and DOG1 in the Diagnosis of Testicular Neoplasms: An Immunohistochemical Study of 95 Cases

$H$ Osman, RE Alexander, L Cheng, TM Ulbright, MT Idrees. Indiana University School of Medicine, Indianapolis, IN.

Background: CDX2 is useful in supporting gastrointestinal origin of tumors in metastatic settings. Similarly DOG-1 and GATA-3 are nuclear markers that are often positive in GISTs and tumors of urothelial and breast origin, respectively. Their expression, however, has not been thoroughly examined in testicular germ cell tumors. Design: We identified 95 testicular tumors (pure and mixed germ cell tumors and sex cord-stromal tumors), including (by predominant neoplastic type): 24 classic seminomas, 21 embryonal carcinomas, 25 yolk sac tumors, 8 choriocarcinomas, 2 primitive neuroectodermal tumors (PNET), 8 Leydig cell tumors and 7 Sertoli cell tumors. Additionally 13 teratomatous areas in foci adjacent to other tumors were also analyzed. Immunohistochemical staining for CDX2, DOG-1 and GATA-3 was conducted, with the cohort limited to 84 cases for DOG1. The distribution, percentage and intensity of positivity were assessed in each case. We also identified the morphological features that were associated with positivity.

Results: CDX-2 was uniformly and strongly positive in all pure yolk sac tumors and yolk sac tumor components of mixed tumors (sensitivity: $100 \%$, specificity: $97 \%$ [teratomas not included]). The positivity appeared to fade with "maturation" within the yolk sac tumor areas. CDX-2 also stained glandular components within teratomas but was negative in all seminomas, embryonal carcinomas, choriocarcinomas \& sex cord-stromal tumors. Interestingly, 6 cases previously diagnosed as pure embryonal carcinomas revealed inconspicuous positive areas for CDX-2 that corresponded to foci with yolk sac tumor morphology on retrospective examination of the H\&E slides. GATA-3 was positive in all choriocarcinomas, staining both mono and multinucleated forms (sensitivity: $100 \%$ ). It also stained scattered syncytiotrophoblast cells seen within other tumors and was patchy positive in the two PNET cases. DOG-1 was negative in all the tumors; however, it was strongly positive in spermatocytes within the adjacent seminiferous tubules.

Conclusions: CDX-2 is a sensitive and, with the exception of teratomatous glands, specific marker for yolk sac tumor. Due to its sensitivity, it may serve as a useful tool to screen for yolk sac tumor components that may be overlooked on H\&E stained slides. GATA-3 is helpful in the recognition of trophoblast cells, especially of intermediate type. DOG-1 appears to be a sensitive marker for spermatocytes and needs to be further studied for its diagnostic utility in the testis.

1039 Translocation Carcinomas of the Kidney Developing within a Short Latency Period after Cytotoxic Chemotherapy for Pediatric Cancer

J Parikh, F Wahid, B McCarvile, A Pappo, A Bahrami. St. Jude Children's Research Hospital, Memphis, TN.

Background: Translocation carcinomas of the kidney (TRCC) are an uncommon category of renal carcinomas often affecting younger individuals. These tumors are genetically defined by translocations involving the MITF/TFE family genes. Most cases develop de novo but a subset may arise after exposure to cytotoxic chemotherapy. From prior reports, the median time to develop TRCC following chemotherapy is 6 years (2.5-13 years).

Design: We searched our institutional files for the diagnosis of TRCC among pediatric cancer survivors to identify potential risk factors and the time latency to develop carcinoma after receiving chemotherapy.

Results: Four cases of TRCC occurred as a second malignancy in pediatric cancer patients. All 4 tumors were detected incidentally; in 2 patients on annual CT bone densitometry and in 2 patients on surveillance abdominal CT scans. All patients received multiple chemotherapy agents, including anthracyclines (4 of 4 ) and platinum-based agents ( 3 of 4). Two patients received radiation (Table 1 ) and 3 patients underwent bone marrow transplantation. All TRCCs developed within 5 years after the completion of chemotherapy (median, 2.3 years). All 4 tumors were $<4 \mathrm{~cm}$ and localized to the kidney (Stage I). On imaging studies, two were sharply defined and two were infiltrative. Histologically, 3 tumors had a predominantly solid and one had a papillary growth pattern. The diagnosis was confirmed by immunohistochemistry for TFE 3 in 3 tumors, of which only one was positive for ASPL-TFE3 fusion transcript by RT-PCR. One tumor was reactive to the TFEB antibody and melanocytic markers.

Conclusions: Chemotherapy-induced translocation renal carcinomas are morphologically and genetically heterogeneous and can develop within a short interval ( $<5$ years) following cytotoxic chemotherapy. 


\begin{tabular}{|c|c|c|c|c|c|c|c|}
\hline Case & $\begin{array}{l}\text { Primary } \\
\text { malignancy }\end{array}$ & $\begin{array}{l}\text { Age at } \\
\text { primary } \\
\text { diagnosis }\end{array}$ & $\begin{array}{l}\text { Interval between } \\
\text { therapy for initial } \\
\text { malignancy and } \\
\text { TRCC }\end{array}$ & $\begin{array}{l}\text { Renal } \\
\text { mass } \\
\text { (size) }\end{array}$ & Prior RT & BMT & TRCC type \\
\hline 1 & ALL & $3 \mathrm{~d}$ & $5-5.5 \mathrm{yr}$ & $1.8 \mathrm{~cm}$ & No & Yes & TFE3 \\
\hline 2 & Neuroblastoma & 1 yo & $11 \mathrm{mo}-2.2 \mathrm{yr}$ & $0.8 \mathrm{~cm}$ & $\begin{array}{l}\text { Yes } \\
\text { (tumor } \\
\text { bed) }\end{array}$ & Yes & TFE3 \\
\hline 3 & Hepatoblastoma & $23 \mathrm{mo}$ & $1.6-2 \mathrm{yr}$ & $2.4 \mathrm{~cm}$ & No & No & TFEB \\
\hline 4 & ALL & 15 yo & $3-3.5 \mathrm{yr}$ & $3.1 \mathrm{~cm}$ & TBI & Yes & ASPL-TFE \\
\hline
\end{tabular}

ALL, acute lymphoblastic leukemia; RT, radiation therapy; TBI, total body irradiation; BMT, bone marrow transplantation

1040 Clinicopathologic Features of Paraganglioma of Urinary Bladde with Emphasis on Malignant Ones: Korean Mulit-Institutional Study

S Park, GY Kwon, SK Kim, YS Chae, JY Kim, KC Moon, YW Park, WY Park, J-Y Sung W Lee, JI Lee, TJ Lee, YM Cho, HY Cho, EJ Cha, G Choi, YJ Choi, JY Ro. Ewha Womans University Mok-dong Hospital, Seoul, Republic of Korea; Other Hospitals, Republic of Korea; The Methodist Hospital, Houston, TX.

Background: Paraganglioma (PG) of the urinary bladder (UB) is a rare neuroendocrine tumor $(<0.05 \%$ of all UB tumors). Only about 180 cases have been reported. Design: To define clinicopathologic features of PG in the UB, a nationwide multicenter study was performed from 17 major institutions in South Korea.

Results: Total 44 cases (M:F=23:21) were assessed. A mean was 56.9 years (range, 22 - 78). Most cases were discovered incidentally (27 cases, 61.4\%) during routine radiographic imaging and the remaining cases presented with hematuria ( 7 cases), voiding difficulty (4), abdominal pain (4), or paroxysmal symptoms with headache, palpitation, tinnitus and excessive sweating after micturition (2). Among 40 of which cystoscopic findings available, the tumor was nodular (22 cases), polypoid (7), submucosal (6), papillary (3), huge mass (1) and unremarkable (1) and measured from 0.5 to $8 \mathrm{~cm}$ (mean, $2.4 \mathrm{~cm}$ ). The tumor was located in the dome (12 cases), lateral (11), posterior (9) and anterior (4) walls, ureterovesical junction (3) and bladder neck (2), and 1 each in anterolateral, posteroinferior wall and trigone. In one case, tumor was located at the perivesical area. Histologic examination showed either nested, zellballen pattern with delicate fibrovascular stroma or solid and diffuse growth pattern. Invasion of muscle proper (MPI) was very common and seen in 31 cases $(70.5 \%)$ with tumor necrosis in 2 and lymphovascular tumor invasion (LVI) in 4 . Mitoses were rare to a few $(<5 / 10 \mathrm{HPFs})$. Immunohistochemistry was performed on 36 cases and all had the characteristic two-cell populations: synaptophysin and chromogranin A positive chief cells and S-100 protein positive sustentacular cells. During 33.3 months of mean followup period (range, 1-166), 3 cases (6.8\%) developed metastatic disease but none of them died of the disease. Compared to the cases with localized to the UB, the metastatic PGs were characterized by a large tumor size (mean, $6.2 \mathrm{~cm}$ vs. $2.0 \mathrm{~cm}$ ) and the presence of necrosis $(33.3 \%$ vs. $2.4 \%)$ and LVI ( $100 \%$ vs. $2.4 \%)$. Among 3 cases with metastasis, metastatic sites were 1 each in paraaortic lymph node (LN), regional $\mathrm{LN}$ and multiple bones. Location, number of mitoses, MPI, clinical symptoms and gross findings were not prognostically significant.

Conclusions: $\mathrm{PG}$ is a rare neoplasm of the UB with a malignant behavior of $6.8 \%$ in our study. Large tumor size, presence of tumor necrosis and LVI are important parameters to predict malignant behavior.

1041 Insulin-Like Growth Factor II mRNA-Binding Protein 3 Expression Promotes Cell Migration through NF-kB Pathway and Predicts Poor Prognosis in Localized Clear Cell Renal Cell Carcinoma

$X$ Pei, MLi, J Zhan, M Zhou, H Zhang, H He. Peking Iniversity, Health Science Center, Beijing, China; Cleveland Clinic, Cleveland, OH.

Background: Insulin like growth factor 2 mRNA binding protein 3 (IMP3) is a novel oncoprotein expressed in embryonic tissues and many cancers. In renal cell carcinoma (RCC) the expression of IMP3 was increased in metastatic RCC and a subset of primary RCC that subsequently developed metastases. Patients with localized RCC that express IMP3 had a shorter survival than those without IMP3 expression. However, the role of IMP3 in RCC progression was poorly understood. In this study, we aim to validate the prognostic significance of IMP-3 of patients with localized CCRCC (clear cell RCC) in a large cohort and explore the mechanism of IMP3 regulating the cell migration. Design: A tissue microarray including 469 clinically localized CCRCC treated surgically between 1990 and 2003 in Cleveland Clinic, US was constructed. The IMP3 expression was analyzed by immunohistochemistry and correlated with the pathologic parameters and survival. The stable overexpression IMP3-Caki-1 and knockdown Achn Sh-IMP3 cell lines were established. Their migration and invasion abilities were measured by transwell assay. RNA sequencing was used to identify different expressing genes in IMP3-Caki-1 cell and the regulating function of NF-KB signaling pathway in cell migration was investigated.

Results: IMP3 was expressed in 73 (16\%) of 469 tumors. Expression was associated with higher nuclear grade, higher T stage, necrosis and sarcomatoid differentiation $(P<$ 0.001). IMP3-positive tumors were associated with shorter recurrence-free survival and overall survival. Multivariable analysis validated IMP-3 as an independent prognostic factor. IMP3 could promote cell motility, migration and invasion in Caki-1 cells. In comparison, knockdown of IMP3 by RNA interference in Achn cells could inhibit cell migration. Overexpression of IMP3 significantly increased the expression of genes involved in NF-kB pathway, such as P50 and P52. Furthermore, overexpression of IMP3 can increase the secretion of IL-8, which is an important downsteam molecule in NF-KB pathway. The ability of IMP3 improving migration of RCC cells was inhibited

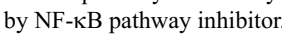

Conclusions: We validate that IMP3 was an independent prognostic marker for localized CCRCC. IMP3 can promote the migration of RCC cells and may function through NF-kB pathway.

1042 Genetic Alterations in Renal Cell Carcinoma with Rhabdoid Differentiation

CM Perrino, V Hucthagowder, S Kulkarni, PA Humphrey. Washington University, Saint Louis, MO.

Background: Rhabdoid renal cell carcinoma is an aggressive variant of kidney cancer. Renal cell carcinoma with rhabdoid differentiation (RD) in adult patients has no known specific genetic alteration. Our aim was to use high density single nucleotide polymorphism (SNP) array to identify genetic aberrations in RD in adults.

Design: A search of the pathology database from 1989 to 2013 identified 56 cases of RD in adult patients. All slides were re-reviewed, and 20 representative cases were selected which displayed both clear cell (CC) and rhabdoid morphology. DNA was extracted from formalin-fixed paraffin-embedded tissue (CC and RD area from each case) and subjected to Affymetrix CytoScan HD array assay. Data was processed and analyzed using Affymetrix Chromosome Analysis Suite v2.0 (2.0.0.195/r5758), BioDiscovery Nexus v7, and Partek Genomics Suite. Genetic aberrations present in $40 \%$ of samples were considered significant.

Results: SNP array was performed on 40 samples ( 20 CC, 20 RD). 30 of these samples passed all quality control measures and were used for data analysis (14 CC and $16 \mathrm{RD}$, 14 of which were pairs from same tumor). Among all CC samples 649 genes had copy number variations (CNV), of which 586 were gains and 66 were losses. Among all RD samples 1,122 genes had CNVs, of which 1,065 were gains and 57 were losses. No chromosomes were exclusively altered in the RD versus the $\mathrm{CC}$ samples. Deletion of chromosome $3 p$ was the only genetic aberration identified in $100 \%$ of $\mathrm{RD}$ and CC components. The following $\mathrm{CNV}$ gains were present in both $\mathrm{CC}$ and RD samples: chromosome 5q (RD 43.8\%, CC 50\%), chromosome 7 (RD 56.3\%, CC 50\%), and chromosome 8q (RB 56.3\%, CC 50\%). The following CNV losses were present in both CC and RD samples: chromosome $3 \mathrm{p}$ (RD 100\%, CC 100\%), chromosome $8 \mathrm{p}$ (RD 68.8\%, CC 42.9\%), and chromosome 14 (RD 62.5\%, CC 57.1\%). RD cases had a higher number of CNVs, with an additional gain of chromosome 1q in RD vs. CC ( $50 \%$ vs. $29 \%$ ) and losses of chromosomes $9(69 \%$ vs. $43 \%)$ and $18(75 \%$ vs. $43 \%)$. Conclusions: Rhabdoid renal cell carcinoma cells share many chromosomal abnormalities with co-existing clear cell carcinoma, including uniform deletion of $3 p$. Overall, rhabdoid cells exhibit a greater number of copy number variations, with several specific chromosomes showing enhanced gain or loss. The greater number of genetic alterations in rhabdoid cells compared to co-existing clear cells, with a shared genetic background of aberrations, suggests genetic evolution of the rhabdoid cells from the clear cells that correlates with the histomorphological progression.

\section{Immunohistochemical Staining for BRAF V600E Supports the} Diagnosis of Metanephric Adenoma

A Pinto, MS Hirsch, S Signoretti, JA Barletta. Brigham and Women's Hospital, Boston, MA.

Background: Metanephric adenoma (MA) is a benign renal tumor that shows histologic overlap with other renal epithelial neoplasms (RENs), such as papillary renal cell carcinoma (PRCC). It was recently demonstrated that the majority ( $90 \%)$ of MAs harbor the BRAF V600E mutation. A BRAF V600E mutation-specific monoclonal antibody (clone VE1) is now commercially available. The aim of this study was to evaluate whether immunohistochemistry (IHC) for BRAF V600E can be used to differentiate MAs from other RENs.

Design: Our cohort included 6 MAs and 146 other RENs, including 109 clear cell renal cell carcinomas, 20 PRCCs, 10 chromophobe renal cell carcinomas, and 7 oncocytomas. IHC for BRAF V600E was performed on whole sections of MAs and on tissue microarrays with all of the other RENs. Moderate to strong granular cytoplasmic staining was scored as positive. No staining (or a weak cytoplasmic or nuclear blush) was scored as negative. $B R A F$ mutational analysis by pyrosequencing had been previously performed on 5 of the 6 MAs. All tested cases had a BRAF V600E mutation.

Results: All 6 MAs $(100 \%)$ demonstrated moderate to strong cytoplasmic staining for BRAF V600E. One PRCC ( $5 \%$ of PRCCs) was positive for BRAF by IHC. All other RENs were negative. $B R A F$ mutational analysis of the one PRCC that was positive for BRAF by IHC demonstrated an underlying $B R A F$ V600E mutation. Cytogenetic analysis of this tumor demonstrated trisomy of chromosomes 7 and 17, confirming the diagnosis of PRCC.

Conclusions: $100 \%$ of MAs in our cohort were positive for BRAF VE1. In contrast, only one PRCC, amounting to $<1 \%$ of all other RENs, was positive for BRAF. Our findings suggest that IHC for BRAF V600E can be used to aid in the diagnosis of MA.

\section{4}

Lichen Sclerosus, a Common Finding in Urethral Strictures in

Males

SV Prabhu, J Liu, CM Gonzalez, XJ Yang. Northwestern University Feinberg School of Medicine, Chicago, IL.

Background: Lichen sclerosus (LS) of the male urethra may be a cause of stricture as well as a potential premalignant condition. Advanced LS requires significant surgical intervention, while early LS can be treated conservatively with topical steroids and other agents. Therefore, early accurate diagnosis is of paramount importance.

Design: Consecutive cases of urethral stricture in males in our institution over a 4.5 year period were retrospectively re-evaluated using 5 histologic features commonly found in LS: (1) hyperkeratosis, (2) thinning or thickening of the epidermis or squamous epithelium, (3) attenuation or vacuolar degeneration of the basal cell layer, (4) subepithelial hyalinization/dermal collagen homogenization, and (5) lichenoid 
lymphoplasmacytic infiltrate. There are currently no standard methods to systematically evaluate LS. Therefore, a new diagnostic scheme was created in which cases with 0 or 1 feature was deemed negative; 2 features, suggestive; and 3 or more features, diagnostic for LS. Clinical follow-up results are being collected.

Results: There were 161 total male urethral stricture specimens. The mean patient age was 48 (range 18-77). Of 121 cases with epithelium available for examination, 20 had 0 criteria of LS (17\%), 40 had 1 (33\%), 27 had $2(22 \%), 16$ had $3(13 \%), 12$ had 4 $(11 \%)$, and 6 had all $5(5 \%)$. Using our new scheme, 60 cases were considered negative $(50 \%), 22$ suggestive $(18 \%)$, and 34 diagnostic $(28 \%)$ for LS. The most predictive histologic criterion for LS was basal layer degeneration ( $70 \%$ of cases with this feature were diagnostic for LS, 23/33), followed by hyperkeratosis $(65 \%, 26 / 40)$, lichenoid inflammation $(61 \%, 28 / 46)$, thickening of the epithelium $(52 \%, 16 / 31)$, subepithelial hyalinization $(50 \%, 22 / 44)$, and thinning of the epithelium $(42 \%, 11 / 26)$. Upon review of the original histopathologic diagnoses, only 25 cases $(21 \%)$ made mention of LS (17 diagnosed, 8 suggestive).

Conclusions: Lichen sclerosus is a common finding in urethral strictures in males with $50 \%$ of cases in this study with evaluable epithelium having at least 2 histologic features, and $28 \%$ with at least 3 . Only about half of these had an original diagnosis that mentioned LS. There are currently no objective methods for pathologic diagnosis of LS. Using a new scheme with more detailed histological characteristics will facilitate better recognition and documentation of LS. Particularly, loss of basal cells and hyperkeratosis are the most predictive criteria for early LS. Additionally, LS may be a pathologically under-recognized entity. We believe our clinical follow-up will validate our pathologic evaluation.

\section{Prostatectomy Findings in Men with "Insignificant" Prostate} Cancer on Biopsy

SV Prabhu, SS Rangarajan, E Gersbach, WJ Catalona, XJ Yang. Northwestern University Feinberg School of Medicine, Chicago, IL.

Background: Over the last 20 years, increased utilization of PSA screening has led to more men being diagnosed with early-stage prostate cancer. Most men with a new prostate cancer diagnosis have localized disease, which has a high curable rate. Some cases may be managed by "active surveillance," which can be undertaken in patients after risk stratification is performed to determine which patients' tumors are least likely to progress. These patients are typically followed by regular PSA level measurement and digital rectal exam with periodic repeat biopsies. We present analysis of the histological characteristics of prostatectomy specimens from 255 patients who met criteria for active surveillance but opted for radical prostatectomy instead. We hope a systematic evaluation of these prostatectomies will lead to a better understanding of the limitations of active surveillance selection criteria.

Design: All outside prostate biopsy cases to our institution during a 4.5 year period were reviewed. Cases eligible for active surveillance were those that fulfilled all of the following criteria by the AUA guideline: PSA of $10 \mathrm{ng} / \mathrm{mL}$ or less, no more than 2 cores positive, no core showing greater than $50 \%$ involvement, and Gleason score $3+3=6$ or less. If a prostatectomy was performed within 6 months, it was evaluated for the following high-risk histologic characteristics: tumor volume $10 \%$ or greater, presence of Gleason score 7 or higher, positive margin, extraprostatic extension, seminal vesicle involvement, and lymph node metastasis.

Results: 1467 outside biopsies were submitted to our institution for presurgical evaluation. Of these, 255 cases contained prostatic adenocarcinoma meeting the criteria for active surveillance, and had subsequent prostatectomy performed at our institution within 6 months. This subset of patients had a mean age of 59 (range 29-75), PSA value of $4.7 \mathrm{ng} / \mathrm{mL}$ (range 0.2-9.4), and prostatic weight of $55 \mathrm{~g}$ (range 15.8-160). Of the 255 prostatectomies, Gleason score 7 or greater was present in 104 cases $(41 \%)$, tumor volume of $10 \%$ or greater in 55 cases $(22 \%)$, positive margin in 20 cases $(8 \%)$, extraprostatic extension in 9 cases $(4 \%)$, seminal vesicle involvement in 2 cases (1\%), and no lymph node metastases. At least one of these high-risk criteria was present in 115 cases $(45 \%)$.

Conclusions: $45 \%$ of prostatectomies contained high-risk histologic features despite eligibility for active surveillance based on the histologic characteristics of the biopsy and PSA value. The clinical significance of these findings in this subset of patients awaits follow-up studies of this patient cohort.

1046 Pathologic Prognostic Variables Determining Disease Specific Outcome in Patients with Metastatic Penile Carcinoma to the Lymph Nodes. A Single Institution Study of 54 Patients

P Rao, PS Fox, P Tamboli, LC Pagliaro, CA Pettaway. MD Anderson Cancer Center, Houston, TX.

Background: Lymph node (LN) involvement in penile cancer has emerged as one of the most powerful prognostic factors of disease outcome over the last few years. The aim of the study was to determine pathologic parameters \& prognostic variables associated with disease specific survival (DSS) in a cohort of LN positive penile carcinoma from a single institution.

Design: We retrospectively reviewed our electronic medical records from 1985-2011 \& identified 244 patients with primary squamous cell carcinoma of the penis. We limited our analysis to LN positive cases for which the lymphadenectomy was performed in house $(n=54)$. Cox proportional hazards regression was performed to assess the association between several covariates \& DSS, which was calculated in months from the initial date of presentation to date of death.

Results: Mean age at presentation was 58 yrs. Stage at time of resection was as follows: pT1: $n=8$, pT2: $n=38$; pT3: $n=7$, pT4 n=1. 38 patients (pts) $(70.4 \%)$ had documented lymphovascular invasion in the primary resection specimen. 21 pts $(38.9 \%)$ had bilateral $\mathrm{LN}$ involvement at the time of resection. Of all the variables examined, the number of positive LNs, LN density \& bilateral LN involvement were significant univariate predictors of DSS. For each increase in the number of positive LNs, the risk of disease specific death increased by $7.4 \%(\mathrm{HR}=1.074, \mathrm{p}=0.0074)$. Metastasis in $>2$ lymph nodes was a predictor of poor DSS. Patients with bilateral nodal disease had a substantial increase in the risk of disease specific death $(\mathrm{HR}=4.12, \mathrm{p}=0.0005)$. In the setting of LN involvement, T stage was not a significant predictor of outcome (overall $\mathrm{p}$ value $=0.39$ ). The median DSS was 35 months \& the 5-year (60 month) DSS rate was $43.2 \%$ (95\% CI: $28.2-57.3 \%)$

Conclusions: Our findings confirm that extent of LN involvement influences outcome in penile carcinoma. Our study suggests that bilateral LN involvement, LN density \& bilateral LN involvement are significant univariate predictors of DSS. In the multivariate model, only LN density remained a significant predictor of worse DSS ( $\mathrm{p}=0.0012)$. In addition, there was some evidence that bilateral $\mathrm{LN}$ involvement was still a significant predictor $(\mathrm{p}<0.10)$. Current analysis is underway to determine the relationship of TNM stage with disease outcome.

\section{Gleason Score 6 Prostate Carcinoma and Presence of Pathological}

\section{Stage T3 Disease}

AA Razmaria, E Negron, GP Zagaja, AL Shalhav, SE Eggener, GP Paner. University of Chicago, Chicago, IL.

Background: Recent studies reassessing large series of prostatectomies with re-review of pathologic specimens substantiate the notion that Gleason pattern 6 graded according to the 2004 International Society of Urological Pathology (ISUP) modified Gleason grading lacks the ability to form metastasis. In this study, we aimed at identifying patients with Gleason score 6 and stage pT3 disease in a large contemporary series at a single institution and carrying out a pathological reevaluation.

Design: From April 2003 to June 2012, 2,659 patients underwent robotic-assisted laparoscopic radical prostatectomy at the authors' institution. Out of this cohort, 37 (1.4\%) cases were identified meeting the inclusion criteria of Gleason score 6 with either extraprostatic extension (34) or seminal vesicle invasion (3). All available pathologic slides of this cohort were re-evaluated by a single subspecialty trained genitourinary pathologist for Gleason grade, pathological stage, extent of extraprostatic extension (EPE, focal or established), and Gleason grade at the site of EPE. Two cases could not be retrieved from the archives ( 1 pT3a and 1 pT3b). The 2004 ISUP modified Gleason grading criteria was applied.

Results: The reviewed cases were from the years 2003 (4), 2004 (9), 2005 (8), 2006 (8), 2007 (2), 2008 (1), 2010 (1), and 2011 (2). Out of the 35 reviewed cases, 27 (77\%) were upgraded to $3+4(25)$ or $4+3$ (2). The majority of cases had only minor components of pattern $4(\leq 5 \%)$ which only under the 2004 ISUP modified Gleason grading system was emphasized to be included as a secondary grade despite the small volume. Three cases $(9 \%)$ were down-staged to pT2 because of only "capsular" incision being present or tumor invading into the "capsule" but not beyond it. Among the cohort, only 5 cases (14\%) with purely Gleason score 6 and pT3a stage were noted. In all these cases, the EPE was focal. Among the re-examined cohort, no cases with purely Gleason score 6 and stage pT3b were observed. In the majority of cases, Gleason grade at the site of EPE was Gleason grade 3 even if grade 4 was present within the prostate. EPE was either via nerve or by direct carcinoma gland extension. However, whenever established EPE was noted concomitant grade 4 was observed at the site of EPE was observed as well. Conclusions: In our large cohort of 2,659 patients, we did not find evidence for presence of seminal vesicle invasion (stage pT3b) with truly Gleason score 6 prostate cancers. Focal EPE in Gleason score 6 cancers is however possible, albeit rare.

\section{Intraoperative Frozen Section Evaluation of Margins in Patients} with Bladder Cancer: Is It Worth It?

NP Reder, SP Maxwell, GA Barkan. Loyola University Stritch School of Medicine, Maywood, IL.

Background: Frozen sections (FS) are used to determine resection margins during radical cystectomy for bladder cancer. Their utility has been questioned, and previous retrospective studies using survival data have demonstrated conflicting results.

Design: A retropective study of 400 patients who underwent radical cystectomy for bladder cancer. Our aims were: 1) to analyze multiple definitions of a "positive" diagnosis for sensitivity, specificity, and positive likelihood ratio (+LR) and 2) to determine the effect of a positive surgical margin (SM) and frozen/permanent section discordance on overall and disease-free survival using clinical follow-up data.

Results: The study cohort had an average age of 67.5 years and was $74.7 \%$ male. There were 189 deaths during the follow-up period and 96 patients had recurrence of disease. The median follow-up time was 27 months. There was a positive correlation between + LR and severity of dysplasia used as a cutoff for a positive diagnosis, with "Carcinoma" displaying the highest +LR (Table 1).

Table 1: Sensitivity, Specificity, and Positive Likelihood Ratio of Frozen Sections

\begin{tabular}{|c|c|c|c|}
\hline \multicolumn{4}{|l|}{$\sqrt{\text { Ureter }(\mathrm{N}=867)}$} \\
\hline Diagnosis & Sensitivity & Specificity & $+\mathrm{LR}$ \\
\hline Mild Dysplasia & 0.85 & 0.96 & 20.79 \\
\hline Moderate Dysplasia & 0.82 & 0.98 & 34.72 \\
\hline Severe Dysplasia / CIS & 0.82 & 0.99 & 63.32 \\
\hline Carcinoma & 0.89 & 0.99 & 122.82 \\
\hline \multicolumn{4}{|l|}{ Urethra $(\mathrm{N}=168)$} \\
\hline Diagnosis & Sensitivity & Specificity & $+\mathrm{LR}$ \\
\hline Mild Dysplasia & 0.75 & 0.93 & 11.10 \\
\hline Moderate Dysplasia & 0.72 & 0.97 & 27.08 \\
\hline Severe Dysplasia / CIS & 0.67 & 0.97 & 25.00 \\
\hline Carcinoma & 0.67 & 0.99 & 106.00 \\
\hline
\end{tabular}

A positive SM $(\mathrm{p}=0.42)$ was not significantly associated with overall survival, nor was frozen/permanent discordance $(\mathrm{p}=0.66)$ when tested in multivariate Cox proportional hazards models (Table 2). Similarly, a positive SM $(p=0.40)$ was not associated with disease-free survival, nor was frozen/permanent discordance $(\mathrm{p}=0.25)$. 
Table 2: Multivariate Cox Proportional Hazards Modeling

\begin{tabular}{|l|l|l||l||}
\hline Overall Survival & HR & $95 \% \mathrm{CI}$ & p-value \\
\hline Variable & 1.12 & $(0.67,1.90)$ & 0.66 \\
\hline Frozen/Permanent Discordance & 1.27 & $(0.71,2.26)$ & 0.42 \\
\hline Positive Surgical Margin & HR & $95 \% \mathrm{CI}$ & p-value \\
\hline Disease-Free Survival & 1.49 & $(0.76,2.94)$ & 0.25 \\
\hline Variable & 0.68 & $(0.28,1.67)$ & 0.40 \\
\hline Frozen/Permanent Discordance &
\end{tabular}

Positive Surgical Margin

involvement.

Conclusions: The results of this study, the first to evaluate the effect of frozen/permanent discordance and the use of different cutoffs for a positive diagnosis, show that FS do not affect patient outcomes in spite of their high sensitivity and specificity. These findings do not support the use of FS during radical cystectomy for bladder cancer.

1049 Urothelial Carcinoma with ERBB2 Mutations: Molecular, Immunohistochemical and Morphologic Analysis

$Q$ Ren, PH Kim, JP Sfakianos, S Scott, G Iyer, AJ Hanrahan, SK Tickoo, SW Fine, A Gopalan, Y-B Chen, JE Rosenberg, DF Bajorin, BH Bochner, VE Reuter, MF Berger, DB Solit, HA Al-Ahmadie. Memorial Sloan-Kettering Cancer Center, NY.

Background: Somatic activating mutations in $E R B B 2$ have been recently identified in a number of tumor types. Aberrations in ERBB2 (amplification and mutations) represent a novel therapeutic avenue for HER2-targeted therapy. HER2 overexpression by immunohistochemistry $(\mathrm{IHC})$ has been used as a reliable surrogate marker for gene amplification in breast and gastric cancers. A minority of urothelial carcinomas (UC) are known to be associated with $E R B B 2$ amplification but little is known about activating mutations in this disease. We identified a cohort of UC with ERBB2 mutations using a next generation sequencing (NGS) platform and performed HER2 IHC for correlation with overexpression.

Design: A total of $138 \mathrm{UC}$ cases were studied. Frozen tumor tissue and matching germline DNA from peripheral blood were analyzed using a targeted, deep-sequencing assay designed to identify point mutations, indels, and copy number alterations in 300 cancer-associated genes, including $E R B B 2$. HER $2 \mathrm{IHC}$ was performed on the 13 cases with confirmed $E R B B 2$ mutations and on 14 randomly selected cases from the cohort that were without $E R B B 2$ mutations or amplification. We compared the staining pattern in these cases with 4 previously identified $U C$ cases known to harbor $E R B B 2$ amplification. Results: $E R B B 2$ somatic mutations were identified in 13 cases. There were 12 missense mutations and one frameshift mutation. Two HER2 mutant tumors exhibited ERBB2 amplification. All 13 cases were of high grade morphology with predominantly solid growth or nodular patterns and associated with a non-invasive papillary component. Detailed results of HER2 IHC are listed in the table.

\begin{tabular}{|l|l|l||l||}
\hline Score & $3+$ & $2+$ & $0-1+$ \\
\hline HER2 mutant UC $(\mathrm{n}=13)$ & $2^{*}$ & 3 & 8 \\
\hline HER2 wild-type UC $(\mathrm{n}=14)$ & 0 & 4 & 10 \\
\hline HER2 amplification UC $(\mathrm{n}=4)$ & 4 & 0 & 0 \\
\hline
\end{tabular}

HER2 amplific

* Tumors containing both HER2 mutations and amplification

Overall, the majority of the HER 2 mutant tumors did not exhibit HER 2 overexpression as determined by intense and diffuse membranous staining by IHC. The only 2 mutant cases with $3+$ staining were associated with HER2 amplification. A significant number of wild type HER2 tumors expressed HER2 at variable intensity but none as $3+$. In comparison, all the cases with known ERBB2 amplification were 3+ by IHC.

Conclusions: A significant minority of urothelial carcinomas has ERBB2 mutations, which may represent targets for anti-HER2 treatment. In rare cases, both mutations and amplification co-occur in the same tumor. IHC is not a sensitive method for identifying tumors with HER2 mutations but remains a sensitive marker for ERBB2 amplification.

1050 Comprehensive Diagnostic and Immunohistochemistry Utilization Data of Prostate Needle Biopsies: A National Pathology Laboratory Experience

AK Riba, AT Savera. PLUS Diagnostics, Union, NJ.

Background: Immunohistochemistry (IHC) is utilized routinely for interpretation of prostate biopsies, particularly for evaluation of 'Atypical Glands Suspicious for Cancer' (ATYP), and in many laboratories PIN4 cocktail is the marker of choice. However, data does not exist regarding utilization trends and diagnostic implications of PIN4 IHC, particularly in the commercial laboratory arena which handles bulk of the prostate biopsies.

Design: We retrieved retrospective data of all the prostate biopsy cases reported during a 12-month period (2012). H\&E and unstained slides (PIN4) were prepared according to our unique sectioning protocols. We performed a comprehensive analysis and also identified all the specimens (sp) for which PIN4 was done and assessed its utilization, utility and diagnostic \& turn-around time (TAT) implications. On a routine basis all the cases with a preliminary diagnosis of ATYP (after work up) had been reviewed in the intradepartmental conference (IC) by a prostate pathologist (PP) and both preliminary and final diagnoses had been recorded.

Results: A total of 7742 prostate biopsy cases $(81,072$ sp) were reported: $43.8 \%$ Benign, $6.5 \%$ HGPIN, $2.8 \%$ ATYP and $46.9 \%$ PC; and GS distribution was: $3+3(33.6 \%), 3+4$ $(30.6 \%), 4+3(11.8 \%), 8(12.6 \%), 9 / 10(11.2 \%)$ and ungraded $(0.2 \%)$. PIN4 IHC was utilized in $2601(33.6 \%)$ cases and $5455(6.7 \%)$ sp - average 2 sp/case. PIN4 was utilized in $19 \%$ Benign, $27 \%$ HGPIN, $97 \%$ ATYP and $42 \%$ of PC cases. PIN4 was performed on $1 \mathrm{sp}$ only in $968(37 \%)$ cases and more than $1 \mathrm{sp}$ in $1633(63 \%)$ cases. PP $(n=2)$ performed PIN4 less frequently as compared to other pathologists $(n=7)$ ( $3.3 \%$ vs $7.2 \% \mathrm{sp}, \mathrm{p}<0.001)$. The diagnosis for individual sp evaluated by PIN4 was: Benign 38\%, HGPIN 9\%, ATYP $8 \%$ and PC $45 \%$. The GS distribution in $2479 \mathrm{PC}$ sp with PIN4 was: $3+3(61 \%), 3+4(21 \%), 4+3(7 \%), 8(7 \%), 9 / 10(3 \%)$, ungraded (1\%).
Of these, $59 \%(1470 / 2479)$ PC were either minimal ( $<5 \%$ core; $43 \% \mathrm{sp})$ or limited $(\leq 10 \%$ core; $16 \% \mathrm{sp})$. Overall, 1155 (6.7\%) cases were reviewed in the IC and 355 of these had a preliminary diagnosis of ATYP which were finally diagnosed as: Benign $(12 \%)$, HGPIN (1\%), ATYP (63\%) and PC (24\%). The mean TAT for cases without PIN4 was 1.75 days vs 2.02 days for cases with PIN4.

Conclusions: PIN4 IHC was employed in $33.6 \%$ of prostate cases with negligible effect on TAT. It was primarily utilized in ATYP cases and in establishing a diagnosis of limited cancer or benign mimicker. The ATYP rate is relatively low (2.8\%), largely attributed to our unique sectioning and intradepartmental review protocols. Morphologic features are paramount in evaluation of ATYP cases; PIN4 is a useful adjunct but has its limitations and pitfalls.

1051 Reclassification of Paratesticular Pseudotumor Based on IgG4 Immunostains

$D W$ Robins, PD Unger, G- $Q$ Xiao. The Icahn School of Medicine at Mount Sinai, New York, NY.

Background: $\operatorname{IgG} 4$ related disease is an incompletely understood process that has a distinct morphology and may involve multiple organ systems. Testicular IgG4 disease, however, has been only scarcely described, with a mere three case reports published (Pubmed search 9/2013). Using the histological features of IgG4 disease as well IgG4 immunostains, we have reclassified cases that were previously diagnosed as paratesticular fibrous lesions or pseudotumors.

Design: A search of our archives for descriptive diagnoses of testicular or paratesticular fibrous/pseudotumor over the past 5 years yielded 6 cases with paraffin-embedded tissue blocks available for further workup. The H\&E slides for each case were reviewed and IgG4 and ALK-1 immunostains were evaluated for each case. Demographic and clinical data were also collected where available.

Results:

\begin{tabular}{|c|c|c|c|c|c|c|c|}
\hline $\begin{array}{l}\text { Case } \\
\#\end{array}$ & $\begin{array}{l}\text { Original } \\
\text { Diagnosis }\end{array}$ & \begin{tabular}{|l} 
Age/Sex \\
and Size of \\
Lesion
\end{tabular} & $\begin{array}{l}\text { Clinical } \\
\text { History }\end{array}$ & \begin{tabular}{|l} 
Storiform \\
Pattern
\end{tabular} & \begin{tabular}{|l} 
IgG4 \\
positive \\
plasma \\
cells \\
per \\
HPF
\end{tabular} & \begin{tabular}{|l} 
ALK-1 \\
IHC
\end{tabular} & \begin{tabular}{|l} 
Revised \\
Diagnosis
\end{tabular} \\
\hline 1 & $\begin{array}{l}\text { Paratesticular } \\
\text { fibrous } \\
\text { pseudotumor }\end{array}$ & $50 / \mathrm{M} 3.0 \mathrm{~cm}$ & $\begin{array}{l}\text { Spermatocele, } \\
\text { Diabetes, BPH }\end{array}$ & Yes & 42 & Negative & $\begin{array}{l}\mathrm{gG} 4 \\
\text { disease } \\
\text { related } \\
\text { lesion }\end{array}$ \\
\hline 2 & $\begin{array}{l}\text { Fibrous } \\
\text { pseudotumor }\end{array}$ & $27 / \mathrm{M} 1.8 \mathrm{~cm}$ & Spermatocele & Yes & $\begin{array}{l}\text { Up to } \\
10^{*}\end{array}$ & Negative & \begin{tabular}{|l} 
gG4 \\
disease \\
related \\
lesion
\end{tabular} \\
\hline 3 & $\begin{array}{l}\text { Inflammatory } \\
\text { fibroxanthomatous } \\
\text { pseudotumor }\end{array}$ & $57 / \mathrm{M} 1.2 \mathrm{~cm}$ & Hydrocele & Yes & $5 *$ & Negative & $\begin{array}{l}\text { IgG4 } \\
\text { disease } \\
\text { related } \\
\text { lesion }\end{array}$ \\
\hline 4 & $\begin{array}{l}\text { Intratesticular } \\
\text { inflammatory } \\
\text { pseudotumor }\end{array}$ & $25 / \mathrm{M} 0.6 \mathrm{~cm}$ & N/A & Yes & 0 & Positive & |---- \\
\hline | 5 & $\begin{array}{l}\text { Paratesticular } \\
\text { hyalized nodule }\end{array}$ & $53 / \mathrm{M} 1.5 \mathrm{~cm}$ & N/A & Yes & 0 & Negative & |----- \\
\hline 6 & $\begin{array}{l}\text { Fibrous } \\
\text { pseudotumor }\end{array}$ & $\begin{array}{l}10 \text { months } / \mathrm{M} \\
0.5 \mathrm{~cm}\end{array}$ & Hydrocele & Yes & 0 & Positive & ----- \\
\hline
\end{tabular}

Note: *Inflammatory cells in Case \#2 and \#3 are almost entirely IgG4 positive plasma cells.

Of the 6 cases with a histological pattern of storiform fibrosis mixed with chronic inflammation, 3 cases stained positively for IgG4 plasma cells (see Table 1). As such, these cases are now reclassified as IgG4 related disease.

Conclusions: Lesions due to IgG4 related disease seem to be rare in the testis / paratesticular region. However, in this case series, half of the previously diagnosed fibrous pseudotumors have been reclassified as IgG4 related disease based on the typical morphology and IgG4 positive plasma cells. Previously it had been thought that paratesticular fibrous pseudotumor resulted from trauma, hydrocele, or spermatocele; the results of this study suggest otherwise. The presence of hydrocele or spermatocele could be secondary to the obstructive mass effect of the fibrous lesion, rather than playing the causative role for the mass.

1052 PCA3 Gene in Prostate Cancer Diagnosis: Improving Biopsy Efficiency and Predicting Tumor Grade and Extension

$N$ Rodon, I Trias, M Verdu, R Roman, A Dominguez, M Calvo, JM Banus, B GarciaPelaez, AM Ballesta, ML Maestro, X Puig. BIOPAT. Biopatología Molecular SL, Barcelona, Spain; Histopat Laboratoris SL, Barcelona, Spain; UB, Barcelona, Spain; ICUN, Barcelona, Spain; Hospital de Barcelona, SCIAS, Grup Assistència, Barcelona, Spain; Analiza. Hospital Moncloa, Madrid, Spain; University Genómica. Hospital Clínico Universitario San Carlos, Madrid, Spain.

Background: PCA3 gene is a molecular biomarker recently incorporated to clinical practice with specificity close to $80 \%$ for prostate cancer (PC) diagnosis. It is currently used as a new diagnostic tool in the clinical management of patients with elevated PSA. PCA3 study in urine collected after prostatic massage significantly reduces the number of prostate biopsies and increases diagnostic yield. Recently it has also granted predictive value of tumor grade and stage. The aim of this study was to analyze the relationship between urine PCA3 score (s-PCA3) with Gleason grade and tumor volume in a series of 131 biopsies performed in patients with elevated PSA and positive s-PCA3.

Design: 750 urine samples collected after prostatic massage, corresponding to 658 patients with elevated PSA, were included. s-PCA3 (ARNmPCA3/ARNmPSAx1000) was determined using Progensa TM kits. Biopsies were performed in patients with positive s-PCA3 $(\geq 35)$. Histopathological and immunohistological studies (AMACR, p63 and 34ß12) were conducted by two pathologists independently, on serial sections of 
paraffin embedded tissues. The average number of studied cylinders was 13 per patient, Gleason scores and percentage of affected cylinders were recorded.

Results: s-PCA3 was positive in $43,3 \%$ of studied samples. In this group 131 biopsies were performed, incidence of PC or atypical small acinar proliferation was $52,7 \%$, reaching to $67,6 \%$ in s-PCA3 $\geq 100$. There is a statistically significant relationship between s-PCA3 and tumor grade $(\mathrm{p}=, 002)$. In cases where positive s-PCA3 was lower than 50 , only $23 \%$ were high grade (Gleason $\geq 7$ ), whereas with s-PCA3 higher than 50 , high grade incidence rose to $75,6 \%$. There was a statistically significant relationship between s-PCA3 and the percentage of affected cylinders $(p=, 023)$. None of the PC with positive s-PCA3 lower than 50 had more than $33 \%$ of the cylinders affected. Both relationships were confirmed by applying the log-linear model that included the three variables.

Conclusions: Including s-PCA3 studies to PC screening allows a significant reduction in the number of biopsies performed $(55,1 \%)$ and an increase of positive biopsies $(52,7 \%)$. s-PCA3 is also an indicator of tumor aggressiveness and provides essential information when making treatment decisions (grade and tumor volume).

\section{Prostatic Involvement by Malignant Lymphoma Is Characterized by Distinct Histologic Features}

FL Rosario-Quinones, PD Unger, GQ Xiao. The Mount Sinai Medical Center, New York, NY.

Background: The incidence of prostatic involvement by malignant lymphoma (ML) has been scarcely described in the literature. When the diagnosis is unknown, lymphoid infiltrates in the prostate can pose a challenge. This is particularly true for low grade lymphomas. We studied the morphologic features that distinguish malignant lymphoid infiltrates from chronic inflammatory processes.

Design: We retrospectively searched the pathology database at our institution from 20002013. Out of 12,880 specimens, 9 cases of prostatic involvement by ML were found. Seven cases were radical prostatectomies (RPs) and 2 were prostate biopsies ( $\mathrm{PBx}$ ). The mean age was 67 years (range, 61-73 years). Three patients had known history of ML, and 6 patients had no known history of hematological malignancies. Five RPs and one chips specimen with findings of chronic prostatitis were used as negative controls. Features evaluated were: cell type, pattern of infiltration, perivascular, perineural and periglandular involvement, and lymphoepithelial (LE) lesions. Clinical information was obtained from the patients' medical records. Diagnosis of all cases was confirmed by immunohistochemical stains.

Results: See Table 1.

\begin{tabular}{|c|c|c|c|c|c|c|c|}
\hline \multirow{2}{*}{\begin{tabular}{|l|} 
\\
Case \\
Num- \\
ber \\
ber
\end{tabular}} & \multirow{2}{*}{\begin{tabular}{|l|} 
Age/Procedure/ \\
Gleason Score
\end{tabular}} & \multirow{2}{*}{$\begin{array}{l}\text { Lymphoid } \\
\text { infiltrate }\end{array}$} & \multicolumn{5}{|c|}{ Histologic Features } \\
\hline & & & \begin{tabular}{|l||} 
Stromal \\
Infiltration
\end{tabular} & \begin{tabular}{|l|} 
Perineural \\
Infiltration
\end{tabular} & \begin{tabular}{|} 
Perivascular \\
Infiltration
\end{tabular} & $\begin{array}{l}\text { Periglandular } \\
\text { Infiltration }\end{array}$ & $\begin{array}{l}\text { LE } \\
\text { Lesions }\end{array}$ \\
\hline & $67 / \mathrm{RP} / 4+3=7$ & SLL/CLL & $\mathrm{Y}$ & $\mathrm{Y}$ & $\mathrm{Y}$ & $\mathrm{Y}$ & Y \\
\hline $2 *$ & $73 / \mathrm{RP} / 4+3=7$ & SLL/CLL & $\mathrm{Y}$ & $\mathrm{Y}$ & $\mathrm{Y}$ & $\mathrm{Y}$ & $\mathrm{Y}$ \\
\hline & $68 / \mathrm{RP} / 3+4=7$ & SLL/CLL & $\mathrm{N}$ & $\mathrm{Y}$ & $\mathrm{Y}$ & $\mathrm{Y}$ & $\mathrm{Y}$ \\
\hline $4^{*}$ & $63 / \mathrm{RP} / 3+4=7$ & SLL/CLL & $\mathrm{Y}$ & $\mathrm{N}$ & $\mathrm{Y}$ & $\mathrm{Y}$ & $\mathrm{Y}$ \\
\hline 5 & $72 / \mathrm{RP} / 3+4=7$ & SLL/CLL & $\mathrm{Y}$ & $\mathrm{Y}$ & $\mathrm{Y}$ & $\mathrm{Y}$ & $\mathrm{Y}$ \\
\hline $6^{*}$ & $63 /$ Prostate bx / & SLL/CLL & $\mathrm{Y}$ & $N$ & Y & $\mathrm{Y}$ & $\mathrm{Y}$ \\
\hline 7 & 68/Prostate bx / & DLBCL & Y & $\mathrm{Y}$ & $\mathrm{Y}$ & $\mathrm{Y}$ & Gland \\
\hline 8 & $61 / \mathrm{RP} / 3+4=7$ & MZL & $Y$ & $N$ & $\bar{Y}$ & $\underline{Y}$ & $\mathrm{Y}$ \\
\hline 9 & $64 / \mathrm{RP} / 3+4=7$ & SLL/CLL & $\mathrm{Y}$ & $N$ & $\mathrm{Y}$ & $Y$ & $\mathrm{Y}$ \\
\hline 10 & $86 /$ Chips/- & $\begin{array}{l}\text { Plasma } \\
\text { cells, L\&H }\end{array}$ & $\mathrm{N}$ & $\mathrm{N}$ & $\mathrm{N}$ & $\mathrm{Y}$ & $\mathrm{N}$ \\
\hline 11 & $62 / \mathrm{RP} / 4+3=7$ & $\mathrm{L \& H}$ & $\mathrm{N}$ & $N$ & $\mathrm{~N}$ & $\mathrm{Y}$ & $\mathrm{N}$ \\
\hline 12 & $49 / \mathrm{RP} / 3+4=7$ & L\&H & $\mathrm{Y}$ & $N$ & $\mathrm{~N}$ & $\mathrm{Y}$ & $N$ \\
\hline 13 & $61 / \mathrm{RP} / 4+3=7$ & $\begin{array}{l}\text { Neutrophils, } \\
\text { L } \mathrm{H}\end{array}$ & $\mathrm{N}$ & $\mathrm{N}$ & Y & $\mathrm{Y}$ & $\mathrm{N}$ \\
\hline 14 & $62 / \mathrm{RP} / 3+4=7$ & L\&H & $\mathrm{N}$ & $\mathrm{N}$ & $\mathrm{N}$ & $\mathrm{Y}$ & $\mathrm{N}$ \\
\hline 15 & $63 / \mathrm{RP} / 3+4=7$ & L\&H & $\mathrm{Y}$ & $\mathrm{N}$ & $\mathrm{N}$ & $\mathrm{Y}$ & $\mathrm{N}$ \\
\hline
\end{tabular}

*previous history of lymphoma was documented. ** $\mathrm{L} \& \mathrm{H}=$ lymphocytes and histiocytes

Conclusions: We described histologic features that distinguish ML from chronic inflammatory infiltrates. ML typically consisted of monotonous lymphocytes with diffuse or patchy distribution, prominent perineural, perivascular, and periglandular involvement with LE lesions. The infiltrating cells in chronic inflammation were a mixture of lymphocytes, plasma cells and histiocytes. Periglandular infiltration without LE lesions was characteristic. ML should be considered in any case where dense lymphocytic infiltrates with these features are identified.

1054 Concurrent Angiomyolipomas and Renal Cell Neoplasms in Patients without Tuberous Sclerosis: A Retrospective Study

LE Rosen, R Ghai, VB Reddy, P Gattuso. Rush University Medical Center, Chicago, IL. Background: Synchronous renal cell neoplasms $(\mathrm{RCN})$ and renal angiomyolipomas (AML) occurring in the same kidney have rarely been reported in the literature. We undertook a retrospective study to investigate the incidence, clinicopathologic features and topographic relationship between renal AML and RCN

Design: Partial and radical nephrectomy specimens with a diagnosis of RCN and AML from 1995 to May 2013 were reviewed. Cases with concurrent AML and RCN were included in this study. The cases were assessed for histologic subtype, topographic relationship between AML and RCN, and clinical characteristics. Melanocytic markers HMB-45 and Melan-A were performed in all concomitant cases of AML and RCN. Results: A total of $565 \mathrm{RCN}$ and 19 AML were identified in our surgical pathology files. The malignant RCN included 331 clear cell, 89 papillary, 24 chromophobe and 5 multilocular renal cell carcinomas (RCC), 5 Wilm's tumors, 2 medullary, 2 collecting duct and 60 urothelial cell carcinomas of the renal pelvis. The benign RCN included 39 oncocytomas, 3 papillary and 3 metanephric adenomas, 1 medullary fibroma, and 1 mesonephroma. Nine of $19(47 \%)$ cases of AML were associated with renal tumors;
8 with RCC and 1 with papillary adenoma. Eight of these patients were female and 1 was male. The age range was 53-87 years with a mean of 66 . The mean age of the 10 patients with AML only was 50 years. Five tumors were located in the right kidney and 4 in the left. The mean size of the AML was $2.2 \mathrm{~cm}$. Histologically, 8/9 AML associated with RCN were triphasic and 1 was smooth muscle predominant. The mean size of the 8 cases of RCC associated with AML was $3.5 \mathrm{~cm}$. Five of 8 cases were clear cell and 3 were papillary carcinomas. The papillary adenoma measured $0.2 \mathrm{~mm}$. All cases of AML associated with RCN occurred in the same kidney but separated from each other. All cases of AML expressed at least one melanocytic marker, Melan-A and or HMB-45. Conclusions: A high percentage of AML (47\%) were associated with RCN. Clear cell carcinoma was the most common malignancy associated with AML. Interestingly, none of the cases of AML were associated with renal oncocytoma. The mean age of patients with concomitant AML and RCN was much higher than the mean age of patients with AML without associated RCN. The majority of AML in non-tuberous sclerosis patients are triphasic tumors and most likely have a benign course. Our study shows that a high number of patients with renal AML ( $42 \%$ ) had concomitant RCC, raising the question if patients with renal AML should have a more aggressive surgical resection.

1055 Next-Generation Sequencing (NGS) Reveals a High Frequency of IHC/FISH Negative Activating Extracellular Domain ERBB2 (HER2) Mutations in Micropapillary Urothelial Carcinoma That Can Respond to Anti-HER2 Targeting Agents

JS Ross, $K$ Wang, LM Gay, RN Al-Rohil, T Nazeer, CE Sheehan, TA Jennings, GA Otto, A Donahue, J He, G Palmer, $S$ Ali, $M$ Nahas, $G$ Young, E LaBrecque, G Frampton, $R$ Erlich, JA Curran, K Brennan, SR Downing, R Yelensky, D Lipson, M Hawryluk, VA Miller, PJ Stephens. Albany Medical College, Albany, NY; Foundation Medicine Inc, Cambridge, MA.

Background: Micropapillary urothelial carcinoma of the urinary bladder (MPUC) is a rare, but highly aggressive form of bladder cancer associated with distant metastases and shortened patient survival. When MPUC recurs and progresses to a systemic disease, there is currently no recognized effective treatment. We conducted genomic analyses (NGS) of MPUC and non-micropapillary urothelial bladder carcinomas (non-MPUC) to characterize the genomic landscape and identify targeted treatment options.

Design: DNA was extracted from 40 microns of FFPE sections from 15 MPUC and 64 non-MPUC tumors. Sequencing (NGS) was performed on hybridization-captured, adaptor ligation based libraries to high coverage for 3,230 exons of 182 cancer-related genes plus 37 introns from 14 genes frequently rearranged in cancer. The MPUC cases were sequenced to at an average depth of 978X. The non-MP UC were sequenced to an average depth of $969 \mathrm{X}$. All samples were evaluated for genomic alterations (GA) including base substitutions, insertions, deletions, copy number alterations (amplifications and homozygous deletions), and select gene fusions/rearrangements. Actionable GA were defined as being linked to commercially available targeted therapies on the market or to targeted therapies being tested registered clinical trials.

Results: Mutations in the extracellular domain of $E R B B 2$ were identified in 6/15 $(40 \%)$ of MPUC: S310F (4 cases), S310Y (1 case) and R157W ( 1 case). All 6 cases of MPUC with $E R B B 2$ mutation were negative for $E R B B 2$ amplification and Erbb2 overexpression. In contrast, 6/64 (9.4\%) non-MPUC harbored an $E R B B 2$ alteration, including base substitution ( 3 cases), amplification ( 2 cases) and gene fusion (1 case), which is higher than the 2/159 (1.3\%) protein-changing ERBB2 mutations reported for urinary tract cancer in COSMIC. The enrichment of ERBB2 alterations in MPUC compared with non-MPUC is significant both between this series $(\mathfrak{p}<0.0084)$ and for all types of urinary tract cancer in COSMIC $(\mathrm{p}<0.001)$. All 9 MPUC cases without $E R B B 2$ mutations harbored at least 1 actionable alteration, including alterations in AKT1, AKT2, CCNDI, EGFR, PIK3CA, PIK3R1 and RAF1.

Conclusions: NGS of MPUC revealed a high incidence of mutation in the extracellular domain of ERBB2 a gene for which there are 5 approved targeted therapies. NGS can identify GA that inform treatment options for the majority of MPUC patients.

1056 Role of SOX2 Amplification, Transcript Level, and Protein Overexpression in Urothelial Carcinoma

S Roy, AM Luvison, KA Fuhrer, KM Cieply, A Karunamurthy, SI Bastacky, R Dhir, AV Parwani. University of Pittsburgh Medical Center, Pittsburgh, PA.

Background: SOX2, a transcription factor, has been implicated in squamous carcinoma (SC) at various sites. More recently, SOX2 protein expression by immunohistochemistry (IHC) and mRNA quantitation by RT-PCR has been shown to be a negative prognostic factor in urothelial carcinoma (UC). We investigated SOX2 gene amplification, in-situ mRNA and protein expression status, correlating with clinicopathologic parameters in UC.

Design: 97 high-grade UCs (HGUC) were retrieved from the case archive after IRB approval and reviewed. , Targets were selected for fluorescence in-situ hybridization (FISH). Dual-color FISH for SOX2 was performed using clone RP11-43F17 (CHORI, Oakland, CA) labeled with Spectrum Orange (Abbott Molecular) and D3Z1 -satellite labeled Spectrum Green (Cytocell aquarius, Cambridge, UK). IHC was performed using rabbit monoclonal antibody against SOX2 (Epitomics, Inc). H-score $>200$ (with $\geq 33 \% 3+$ cells) was a surrogate for protein overexpression. In brief, FFPE slides were baked, deparaffinized, conditioned and run on the Discovery Ultra (Ventana, Tucson, AZ) using the RNAscope probe VS Hs-SOX2 (ACD, Hayward, CA).

Results: Of 97 HGUC, 13 were non-invasive and 84 were invasive $(78.6 \%$ were muscle invasive). Histologic subtypes included NOS, squamous, micropapillary, and neuroendocrine. Amplification of SOX2 was an infrequent (7/97) and low level (mean SNR 2.27). Only 2 of the 7 SOX 2 amplified cases had protein overexpression Overall protein overexpression (mean H-score 253) was seen in $13.1 \%$ cases of invasive UC (at least pT2), $73 \%$ of which were high T stage with nodal disease. No pT1 tumors had SOX2 overexpression or amplification. SOX2 overexpression correlated with 
neuroendocrine histology and perineural invasion only $(\mathfrak{p}<0.0001$ and $\mathfrak{p}=0.01$, Fischer's exact). Ten of 47 cases with UC in-situ with or without invasive UC showed SOX2 overexpression. Preliminary data from RNAScope analysis demonstrated marked increase in SOX2 transcript levels in cases with protein overexpression. Outcome analysis and correlation with SOX2 expression is currently in progress.

Conclusions: SOX2 gene amplification or protein overexpression does not appear to be a major driver of urotheial carcinogenesis with the exception of neuroendocrine variant where protein overexpression was consistently increased. In-situ mRNA analysis suggests that in a subset of cases (in contrast to lung $\mathrm{SC}$ ), SOX2 overexpression is primarily driven by increased SOX2 transcript stability rather than gene amplification. This may implicate potential role of SOX2 transcript regulatory mechanisms in UC.

1057 High-Resolution Single Nucleotide Polymorphism (SNP) Microarray Profiling of Urothelial Carcinoma and Corresponding Metastasis

S Roy, C Deamont, A Geskin, P Petrosko, M Lyons-Weiler, R Dhir, SI Bastacky, GK Michalopoulos, WA LaFramboise, AV Parwani. University of Pittsburgh Medical Center, Pittsburgh, PA.

Background: A subset of high-grade urothelial carcinomas (HGUC) have the propensity for metastatic disease. Currently, no biomarkers are available to predict aggressive HGUC. We investigated genome wide alterations in primary and metastatic HGUC to identify potential prognostic biomarkers

Design: 7 cases of muscle invasive HGUC with nodal metastasis and matched normal (benign pelvic lymph nodes (LNs)) were evaluated. Genomic DNA was purified from formalin-fixed, paraffin embedded (FFPE) primary and LN metastatic HGUC and benign LNs using a modified protocol for the QiaAmp DNA FFPE Tissue Kit (Qiagen, Valencia, CA). Spectrophotometric analysis (NanoDrop, Wilmington, DE) and Agilent Bioanalyzer 12000 DNA chip (50ng/ul) were performed to assess DNA quality and integrity. SNP analysis was performed using a Affymetrix Genome-Wide SNP 6.0 protocol (Affymetrix, Sunnyvale, CA following restriction digestion, ligation, PCR amplification, purification and fragmentation. The arrays were washed, stained and scanned (Affymetrix Gene Chip Fluidics Station) . Partek Genomics Suite software (Partek Inc., St Louis, MO) was used for copy number analysis and to identify regions of copy number variation from normal, using a minimum of 20 genomic markers and $p<0.0005$, with signal to noise set at 0.7 and expected range 1.7 to 2.3 . Human genome assembly hg 19 was used to annotate each segment.

Results: 2620 copy number alterations (CNA) occurred in primary HGUCs (1289 amplifications, 1331 deletions). Specific regions of chromosomes 8 (8q11.1-11.23, $8 \mathrm{q} 22.2-8 \mathrm{q} 22.3,8 \mathrm{q} 24.21)$ and $20(20 \mathrm{q} 13.13-20 \mathrm{q} 13.33)$ showed amplifications in as many as $5 / 7$ HGUC. Several protein and miRNA coding genes map to these regions (c- $M Y C$, PCATI, CEBPD, SALL4, GNAS, mir 1204, mirl205, and mir 1206). Deletions in at least $5 / 7$ cases occurred in 15q13.3 region. The number of CNA and amplifications in LN metastases was significantly lower ( 1200 total, $\mathrm{p}<0.05$; 547 amplifications, $\mathrm{p}<0.05$; paired t-test). Total deletions were lower in metastases, but failed to reach statistical significance (653, $\mathrm{p}=0.09$, paired t-test). Comparative analysis revealed shared CNA between primary and metastatic HGUC in 20q13.12-13.2 (amplifications) and 8p23.123.3 (deletions) regions.

Conclusions: 1) Most frequent CNAs occurred in relatively small regions of $8 \mathrm{q}$ and 20q in HGUC. 2) Global genomic alterations in metastatic HGUC is significantly lower than corresponding primary tumor. 3) Common CNAs present in primary and metastatic HGUC suggest possible biomarkers of metastatic potential.

1058 Tumor Recurrence in Neobladder - Urine Cytology Findings with Long-Term Follow-Up

S Roy, SE Monaco, L Pantanowitz, WE Khalbuss. University of Pittsburgh Medical Center, Pittsburgh, PA.

Background: Urinary diversion by ileal conduits is an important reconstructive surgery in patients undergoing radical cystectomy. These patients are followed up by urine cytology screening due to the risk of recurrent disease in the upper urinary tract. Recurrence in the conduit is rare. We investigated clinical and cytological features of tumor recurrences in neobladder urine specimens at our institution.

Design: A total of 450 consecutive archival neobladder urine specimens were retrieved over a period of 5.5 years. At the time of final interpretation, Papanicolaou-stained, ThinPrep or cytospin slides were reviewed along with H\&E-stained sections from available cell blocks. Demographic parameters, clinical information, cytologic diagnoses, detailed cytomorphological features and available follow-up (subsequent urine cytology or corresponding histopathology) was documented.

Results: For 22/450 cases (4.9\%) neobladder cytology specimens from 14 patients had a positive or indeterminate (atypical/suspicious) diagnosis. All 14 patients had a history of invasive high-grade urothelial carcinoma (HGUC), including 8 (57\%) with muscle invasion. All patients were followed with urine cytology (median follow-up 54 months). 4/14 (29\%) patients with abnormal urine cytology (1 atypical, 3 suspicious) had recurrent HGUC confirmed by histopathology. Cytomorphologic findings in cases with negative cytologic diagnoses showed predominately degenerating intestinal epithelial cells with eosinophilic intracytoplasmic inclusions in a degenerated background. The remaining cases (especially suspicious or positive) had atypical urothelial cells with high N/C ratios, enlarged hyperchromatic nuclei, occasional prominent nucleoli, and irregular nuclear contours in a degenerated background.

Conclusions: Recurrent urothelial carcinoma detected in neobladder urine specimens by cytology in our cohort study was rare. Recurrent tumor was characterized by cytological features of HGUC and a markedly degenerated background. Since management of patients with abnormal neobladder cytology may not include a follow-up biopsy, accurate diagnosis is important and challenging.
1059 Grade Migration of Non-Invasive Papillary Urothelial Carcinomas of the Urinary Bladder Following Implementation of the 2004 ISUP-WHO Classification System. A Single Institution Analysis

$R$ Ruiz-Cordero, S Lokeshwar, MS Soloway, S Umar, M Jorda. University of Miami Hospital / Jackson Memorial Hospital, Miami, FL.

Background: The 1973 WHO grading system of urothelial carcinoma (UCa) is a 3-tier grading system, (grade 1,2 and 3) currently not in use, mainly due to low inter-observer reproducibility. In 2004, the WHO and the international society of urological pathology (ISUP) developed a 2-tier grading system for UCa with the goal of providing more accurate information for patient management. This grading system classifies tumors into low-grade (LG) and high-grade (HG) carcinomas, eliminating the previous grade 2. The aim of this study is to investigate if there is a grade migration of non-invasive $\mathrm{UCa}$ of the urinary bladder (UB) after implementation of 2004 WHO-ISUP grading system. Design: The database of 1,175 UCa of the UB diagnosed in a single institution from 2004 to 2012 was reviewed. Grading and invasion, as well as depth of invasion when present, were noted. Linear regression analysis weighted by number of cases per year was performed to determine the percentage change in frequency of diagnosis of LG and $\mathrm{HG} \mathrm{UCa}$.

Results: The 1,175 cases were classified as CIS (102, $8 \%$ ), Non invasive UCa (Ta) (465, $40 \%$ ), Invasive $\mathrm{UCa}$ to subepithelial connective tissue (T1) $(247,21 \%)$, and Invasive $\mathrm{UCa}$ to muscularis propria or beyond $(\geq \mathrm{T} 2)(361,31 \%)$. Of the $465 \mathrm{Ta} \mathrm{UCa}, 212(45 \%)$ were diagnosed as LG and $253(55 \%)$ as HG. There was an overall decrease of $46 \%$ in the diagnosis of Ta-LG UCa, as well as an overall increase of $57 \%$ in the number of Ta HG $\mathrm{UCa}$ during the 9 -year study period. Weighted linear regression analysis demonstrated an average progressive increase in the frequency of cases diagnosed as Ta-HG UCa of $7 \%$ per year $\left(\mathrm{p}=0.00003, \mathrm{R}^{2}=0.85\right)$. The number of cases per year of $\mathrm{T} 1, \mathrm{CIS}$, and $\geq \mathrm{T} 2$ did not show a significant change throughout the study period.

Conclusions: This retrospective study demonstrates a progressive increase in the diagnosis of Ta-HG tumors. On the other hand, the number of Ta-LG UCa showed a constant decrease. This increase in Ta-HG tumors could be secondary to the implementation of the 2004 ISUP-WHO system, as the majority of prior grade 2 tumors are now categorized into the high-grade bracket. Since current guidelines for the treatment and follow up of LG UCa patients differ from those of patients with HG $\mathrm{UCa}$, this grade migration may have an impact in treatment and patient management.

1060 Influence of Chronic Exposure to Persistent Low Dose Irradiation (CPLDIR) in the Angiogenesis of Renal Clear Cell Carcinoma. An Immunohistochemical and Morphometric Study

A Ruiz-Sauri, G Valencia-Villa, A Romanenko, J Perez, $R$ Garcia, A LlombartBosch. Valencia University Medical School, Valencia, Spain; Universidad del Norte, Barranquilla, Colombia; Institute of Urology, Kiev, Ukraine; Universidad Libre, Baranquilla, Colombia

Background: Renal clear cell carcinoma (RCCC) is the most frequent malignant carcinoma of this organ. Tumor angiogenesis is a crucial factor for growth and metastasis, but the significance of this process remains controversial in RCCC. We have described higher incidence and malignancy of RCCC exposed to chronic persistent low dose irradiation (CPLDIR) in contaminated regions of Ukraine as a late effect after the Chernobyl accident. Herein we communicate the intratumoral and peritumoral microvascular density of tumors and the immunohistochemical expression of endothelial factors and their receptors involved in vascular angiogenesis. For comparative purposes we have included RCCCs originating from two irradiation-free countries: Spain (Valencia) and Colombia (Barranquilla)

Design: TMAs were constructed from paraffin blocks of RCCC and peritumoral tissues of 124 patients ( 25 from Spain, 24 from Colombia, 50 from the contaminated area of Ukraine and 25 from the clean area of Ukraine). 50 renal samples without pathology served as control. Antibodies tested: CD 31, VEGF, FGF-2, HIF-1 $\alpha$, VE-cadherin, PDGFR, VEGFR-1,2, and 3 (DAKO Envision and Santa Cruz Biotechnology) Quantitative morphometric studies: image analysis with two different semiautomatic methods: the value of the MVD with the Image Pro Plus 7.0 (Infaimon, Media Cybernetics) and the expression of the factors involved in angiogenesis by quantification using the densitometric Panoramic Viewer 1.15.

Results: Both vascular density (MVD) as well VEFG expression of RCCC results incremented exponentially in direct relation to the level of persistent chronic exposure to low doses of irradiation in the Ukrainian patients seen up to 25 years after the Chernobyl accident, when compared to the other two groups of renal carcinomas studied, belonging to non irradiated areas (Valencia and Barranquilla).

Conclusions: CPLDIR significantly influences the biology of RCCC by means of increased vasculature, promoting a higher aggressiveness with respect to the size of the tumor and the histological grade.

1061 Correlation of Neuroendocrine Differentiation and Androgen Receptor Status in Prostate Adenocarcinoma

ET Sadimin, GT Lee, MS May, IY Kim. Rutgers Robert Wood Johnson Medical School, New Brunswick, NJ; Rutgers Cancer Institute of New Jersey, New Brunswick, N. Background: It is generally believed that cells with neuroendocrine differentiation in prostate adenocarcinoma are androgen receptor negative and therefore contribute to the insensitivity of the tumor to androgen stimulation. However, recent studies have raised the possibility that a group of neuroendocrine cells in prostate cancer may be able to co-express androgen receptor. In this study, we utilized immunofluorescence dual staining technique to compare the androgen receptor expression of neuroendocrine cells in normal prostate versus those in low grade and high grade prostate adenocarcinoma. Design: Two Androgen Receptor (AR) antibodies were used in the study; Santa Cruz AR Antibody C-19 (SC-815 donkey anti-rabbit polyclonal,1:50) and Santa Cruz AR 
Antibody N-20 (SC-816, donkey anti-goat polyclonal, 1:50). For neuroendocrine marker, we used Ventana Chromogranin A (donkey anti-mouse monoclonal, predilute). The red fluorophores (AlexaFluor 555) for both Santa Cruz AR antibody C-19 (donkey anti-rabbit, 1:300) and Santa Cruz AR antibody N-20 (donkey anti-goat, 1:250) were visualized using TRITC filter. The green fluorophore (AlexaFluor 488) for Ventana Chromogranin A (donkey anti-mouse, 1:250) was visualized using FITC filter.

Results: In cells positive for androgen receptor, both Santa Cruz AR antibodies were expressed within the nucleus, cytoplasm or both nucleus and cytoplasm. The $\mathrm{C}$ terminal antibody showed stronger cytoplasmic staining compared to the $\mathrm{N}$ terminal antibody. In our study, all of the neuroendocrine cells in normal glands did not co-express androgen receptor. While there were few neuroendocrine cells in Gleason grade 3 adenocarcinoma co-expressed androgen receptor, there were even more neuroendocrine cells in Gleason grade 4 adenocarcinoma that co-expressed androgen receptor.

Conclusions: We have shown in this preliminary study that, indeed, some neuroendocrine cells in prostate adenocarcinoma can co-express androgen receptor. These cells were more common in higher grade prostate adenocarcinoma. As the next step of the study, we will utilize tissue microarrays of prostate adenocarcinoma, so that a statistical analysis could be performed on the results obtained from a larger quantity of specimens. This study contributes to further understanding of castration resistant prostate adenocarcinoma.

1062 Histopathologic Review of 310 Radical Cystectomy and Lymph Node Dissection with Emphasis on Histologic Variants of Urothelial Carcinoma

K Salazar, M Cookson, G Giannico, O Hameed, LL Gellert. Vanderbilt University Medical Center, Nashville, TN; University of Oklahoma Health Sciences Center, Oklahoma City, OK.

Background: Urothelial carcinoma has a marked propensity for divergent differentiation. Histologic variants of urothelial carcinoma are clinically significant at diagnostic, therapeutic and prognostic levels. Few studies have described the prevalence and extent of such variants in the bladder and associated metastases. In this study, we systematically characterized the histologic variants in a large series of primary bladder urothelial carcinoma as well as their lymph node (LN) metastases.

Design: 310 consecutive cases of radical cystectomy and $\mathrm{LN}$ dissection over a 4-year period performed at a single institution were reviewed by GU pathologist(s). Strict diagnostic criteria are used for each histologic variant, such as "unequivocal keratin formation and/or intercellular bridges" for squamous differentiation and "multiple small nests in the same lacunar space" for micropapillary features. Clinical data were collected from patient charts.

Results: Of the 310 cases, $32 \%$ (100/310) exhibits variant morphology at least focally in the tumor, including $11 \%$ squamous, $6 \%$ micropapillary (MP), $5 \%$ glandular, $4 \%$ sarcomatoid, $2 \%$ diffuse/plasmacytoid, $2 \%$ neuroendocrine/small cell and $2 \%$ others. $4 \%$ of the cases displayed more than one variant morphology. LN metastases were present in $38 \%(38 / 100)$ of the cases with variant morphology vs. $17 \%(36 / 210)$ of the remainder ( $\mathrm{p}=0.0001$, multivariant analysis pending). Concordant variant morphology is present in LN metastases. 13 of the 20 tumors with MP features had LN metastases, and 9 of them exhibited MP features in LN. Of the 4 cases without variant morphology in LN, 3 cases had MP features in $<5 \%$ of the primary tumor. 13 of the 34 tumors with squamous features had LN metastases, and 9 of them exhibited squamous features in LN. No correlation between the presence of squamous features in LN and the extent of such feature in the primary tumor was identified.

Conclusions: A significant proportion of bladder urothelial carcinoma show at least focal divergent differentiation, which often replicated in their $\mathrm{LN}$ metastases when present. The presence of the variant morphology in LN metastases is associated with the extent of such morphology in primary tumor for MP, but not squamous differentiation. Further clinical followup and multivariant analysis are ongoing to thoroughly characterize the correlation between histologic variants and LN metastases as well as their clinical significance.

\section{Prostate Needle Biopsy Is Predictive of Neurovascular Bundle} Involvement in Radical Prostatectomy

H Samaratunga, J Yaxley, B Delahunt. Aquesta Pathology, Brisbane, Queensland, Australia; Royal Brisbane Hospital, Brisbane, Queensland, Australia; Wellington School of Medicine and Health Sciences, University of Otago - Wellington, Wellington, Otago, New Zealand.

Background: Prior to radical prostatectomy (RP), the risk of neurovascular bundle (NVB) involvement by carcinoma needs to be assessed to enable decisions regarding NVB preservation. The value of needle biopsy findings in predicting NVB involvement has not been adequately studied.

Design: Analysis of clinical and pathological data of 472 consecutive RP specimens revealed 167 patients who had one or both NVBs resected. Of these, 122 patients (176 NVB resected) had prior prostate biopsies in our institution. RP specimens were entirely embedded and evaluated by routine microscopy. Tumors were graded using the ISUP modified Gleason system and staged according to ISUP recommendations. Extraprostatic extension at the NVB was considered NVB involvement.

Results: 51 patients had carcinomatous involvement of one or both NVBs with 57 NVBs positive for carcinoma. 71 patients had no NVB involvement. Of the $176 \mathrm{NVBs}$ resected, 119 were not involved. The mean age of patients with NVB involvement was 62.2 years (range 44-77yrs) versus 63.4years (50-79yrs) for no NVB involvement. The mean preoperative PSA was $6.6 \mathrm{ng} / \mathrm{mL}$ (range $0.8-15$ ) \& $7.4 \mathrm{ng} / \mathrm{mL}$ (range 1.5-33) respectively. Pre-operative clinical stage cT2 was found in $21.5 \%$ of positive NVB group vs $20 \%$ of negative NVB group. No patient had pre-op cT3 disease. In the group with NVB involvement $89.5 \%$ had a Gleason score (GS) of $\geq 4+3$ with $5+4 / 4+5 / 5+5=10$ in $33(57.9 \%) 4+4$ in $5(8.8 \%), 4+3$ in $13(22.8 \%)$ and $3+4$ in $6(10.5 \%)$ In contrast, only $37.7 \%$ of patients without NVB involvement had a GS of $\geq 4+3$ with $5+4 / 4+5$ in $19(15.9 \%) 4+4$ in $8(6.7 \%) 4+3$ in $18(15.1 \%), 3+4$ in $45(37.9 \%), 3+3$ in $13(10.9 \%)$ and no cancer in $16(13.5 \%)$. The fraction of positive cores was $<1 / 3$ in $2(3.5 \%)$ and 54 $(45.4 \%)$ in the NVB positive and negative sides respectively. In 6 patients with NVB involvement and GS $3+4$ cancer, the fraction of positive cores in all patients was $\geq 50 \%$. In this patient cohort, $36 \%$ (19 of 52) with GS $9-10,61.5 \%$ with GS $8(8 / 13)$, and $58 \%$ with GS 4+3 (18/31) had no NVB involvement.

Conclusions: All patients with NVB involvement had needle biopsies positive for cancer, which was high-grade in $89.5 \%$. Patients with GS $<4+3$ prostatic adenocarcinoma on needle biopsy, appear to have a lesser risk of NVB involvement unless $>50 \%$ of the cores are positive. Also, some men with high grade disease may not need resection of their NVB despite having high risk disease.

1064 Non-Invasive Micropapillary Urothelial Carcinoma: A Clinicopathologic Review of 12 Cases

AR Sangoi, RM Cox, JP Higgins, RV Rouse, JK McKenney. El Camino Hospital, Mountain View, CA; University of Arkansas for Medical Sciences, Little Rock, AR; Stanford Medical Center, Stanford, CA; Palo Alto Veterans Hospital, Palo Alto, CA; Cleveland Clinic, Cleveland, $\mathrm{OH}$.

Background: "Micropapillary carcinoma" (MPC) typically connotes an invasive pattern of urothelial carcinoma with well-recognized aggressive behavior and poor outcome. Only one prior study has evaluated non-invasive MPC. We evaluate the clinicopathologic features in an additional series of non-invasive urothelial MPC with an emphasis on clinical outcome.

Design: 12 cases of non-invasive MPC (pTa) were reviewed with percent of noninvasive MPC as well any non-traditional pTa carcinoma patterns scored. MPC was defined as secondary papillae arising from the main papillary stalk with a length 5 times greater than the width. Cases with any degree of invasion, regardless of extent, at the time of TURBT or by previous history were excluded. Clinical follow-up was obtained. Results: All 12 patients were males with ages ranging from 55-86y (mean: 70.5y) and all tumors were from the urinary bladder. Non-invasive MPC comprised from $5-95 \%$ of the tumor (mean 34\%). Non-invasive cribriform pattern was also seen in 8 cases $(5-70 \%$, mean $22 \%)$ while villoglandular pattern was seen in 5 cases $(5-60 \%$, mean $26 \%) .6$ cases presented with hematuria, 1 as an incidental tumor, and 5 unknown. Treatment data available for 11 cases included: surveillance $(\mathrm{n}=8)$, cystoprostatectomy $(\mathrm{n}=1), \mathrm{BCG}+$ mitomycin $(\mathrm{n}=1)$, and $\mathrm{BCG}(\mathrm{n}=1)$. Follow-up data was available for 11 cases (range 3-121 months, mean $=47$ months): no new occurrences to date $(n=8)$, stage progression to $\mathrm{pT} 1$ papillary urothelial carcinoma $(\mathrm{n}=2)$, and died of other disease $(\mathrm{n}=1)$. Conclusions: This study suggests that non-invasive MPC of the urinary bladder is often associated with non-invasive cribriform and villoglandular patterns and is not necessarily a high risk carcinoma that warrants immediate definitive surgical therapy.

\section{Prostate Carcinoma in Renal Transplant Recipients: A Single Centre} Experience

R Santi, D Villari, S Caroassai Grisanti, M Zanazzi, G Nicita, G Nesi. University of Florence, Florence, Italy; Careggi University Hospital, Florence, Italy.

Background: Renal transplant recipients are known to be at a greater risk for de novo urological malignancies than the general population. Several site-specific risk factors have been implicated with prolonged immunosuppressive therapy playing a major role. The incidence and treatment of prostate cancer ( $\mathrm{PCa}$ ) in renal transplant patients have not been extensively evaluated.

Design: We conducted a retrospective review of clinico-pathological data from 535 male patients subjected to kidney transplantation at our institution between 1991 and 2013, in order to evaluate the prevalence, management and outcome of PCa in this population. Results: PCa was diagnosed in 15/535 $(2.8 \%)$ recipients without a prior history of prostate malignancy. Mean age at transplantation was 55.9 years (median: 58 years; range: $40-71$ years). Mean age at $\mathrm{PCa}$ diagnosis was 63.5 years (median: 64 years; range: 50-72 years). Biopsy Gleason score was 6 in 9/15 (60\%) patients, 7 in 4/15 (26.7\%) and 8 in $2 / 15$ (13.3\%). At the time of PCa diagnosis, all patients had functioning grafts. Eleven patients underwent radical prostatectomy, three patients were treated with a combination of androgen ablation and external radiotherapy, whilst one patient was closely monitored using the watchful waiting approach. Surgical Gleason score was 6 in $6 / 11(54.5 \%)$ patients, 7 in 1/11 (9.1\%), 8 in 1/11 (9.1\%), 9 in 1/11 (9.1\%). In two patients $(18.2 \%)$ receiving neoadjuvant hormonotherapy, Gleason score was not assessed. According to the 2010 TNM classification, the pathological disease stage was $p T 2 a$ in $1 / 11(9.1 \%), p T 2 c$ in $7 / 11(63.6 \%), p T 3 a$ in $2(18.2 \%)$ and $p T 3 b$ in $1(9.1 \%)$. Mean follow-up was 51.6 months (median: 32 months; range: $4-170$ months). Overall survival rate was $35 \%$. Three patients died from causes unrelated to $\mathrm{PCa}$, whereas one patient died following PCa progression (cancer specific death rate: $6.6 \%$ ). One patient experienced chronic renal allograft rejection and started dialysis. Four of the 15 PCa patients $(26.7 \%)$ had associated skin cancers (squamous cell carcinoma and basal cell carcinoma)

Conclusions: Our results indicate an incidence of $\mathrm{PCa}$ in renal transplant recipients similar to that reported in the general population. In this clinical setting, radical prostatectomy and radiotherapy were safe and effective treatment modalities without compromise in graft function.

1066 Clinicopathological Analysis of Prostatic Adenocarcinoma at Anterior Location in Japanese Men

S Sato, H Takahashi, B Furusato, M Okayasu, M Ikegami. The Jikei University School of Medicine, Minato-ku, Tokyo-to, Japan.

Background: We have shown striking cancer dominance at transition zone (TZ) in Japanese prostate than American (41.8\% in Japanese vs. $0.6 \%$ in American) at 2013 
USCAP meeting. Based on this, circumstantial clinicopathological analysis was performed about TZ/anterior PCa and compared with $\mathrm{PZ} /$ posterior cancer.

Design: A total of 201 cases of radical prostatectomy were utilized. In this research, more practical way to designate cancer location was adopted: cases were divided with a dominant location as anterior (Ant) or posterior (Post) with more than $2 / 3$ of the tumor existence in the area. Cases not fit in this criterion were evaluated as no dominancy (ND). Same cases were divided as TZ or peripheral zone (PZ) cancers as previously. Gleason score (GS), pT, pN, extraprostatic extension (EPE), lymphovascular invasion (LVI) were compared.

Results: Cases were divided as 84, 71, and 46 of Ant, Post, and ND; 85 and 115 of $\mathrm{TZ}$ and PZ, respectively. The GS distribution was shown in Table 1 and no significant difference was shown between Ant and Post $(\mathrm{p}=0.070)$. The results were similar between $\mathrm{TZ}$ and PZ cancers $(\mathrm{p}=0.42)$. EPE status distribution was shown in Table 2: significant numbers of EPEx were included in Ant cases $(\mathrm{p}=0.0035)$. Associated with this, pT distributed as T2, 3, and $2+$ in $45(53.6 \%), 24(28.6 \%)$, and $15(17.9 \%)$ in Ant; 23 (32.4\%), 46 (64.8\%), and $2(2.8 \%)$ in Post, respectively ( $\mathrm{p}=0.000097)$. The results were similar among $\mathrm{TZ}$ and $\mathrm{PZ}$ cancers, although the significance was more marginal $(\mathrm{p}=0.032 / 0.028)$. The LVI distributed as 0,1 in $76(90.5 \%)$ and $8(9.5 \%)$ in Ant; $44(62.0 \%), 26(36.6 \%)$ in Post, respectively. Ant cancer showed significantly lower frequency of LVI than Post $(\mathrm{p}=0.000044)$, although, there were no significant differences in $\mathrm{pN}$ status.

Gleason score (Ant vs. Post)
\begin{tabular}{|l|l|l|l|}
\hline Gleason score & Ant & Post & ND \\
\hline 6 & $13(15.5 \%)$ & $6(8.5 \%)$ & $6(13.0 \%)$ \\
\hline 7 & $58(69.0 \%)$ & $44(62.0 \%)$ & $31(67.4 \%)$ \\
\hline $8-10$ & $13(15.5 \%)$ & $21(29.6 \%)$ & $9(19.6 \%)$ \\
\hline total & 84 & 71 & 46 \\
\hline
\end{tabular}

\begin{tabular}{|c|c|c|c|}
\hline EPE & Ant & Post & ND \\
\hline 0 & $46(54.8 \%)$ & $24(33.8 \%)$ & $23(50.0 \%)$ \\
\hline 1 & $23(27.4 \%)$ & $45(63.4 \%)$ & $17(37.0 \%)$ \\
\hline $\mathrm{X}$ & $15(17.9 \%)$ & $2(2.8 \%)$ & $6(13.0 \%)$ \\
\hline total & 84 & 71 & 46 \\
\hline
\end{tabular}

Conclusions: Ant cases included a significant numbers of high GS and EPEx/pT2+, suggesting a clinical importance and a difficulty of complete surgical resection at the retro-pubic area. Clinically significant cancers are located in anterior portion of the prostate in Japanese men. Accordingly, different methods of prostate biopsy and surgical procedures may need to be considered for cancer management in Japan. A similar verification will be recommended in other countries because the cancer location may be different among races or regions.

1067 Expert GU Pathology Consultation Data: Concordance Analysis of Cases from In-Office Pathology Labs

AT Savera. PLUS Diagnostics, Union, NJ.

Background: During the last decade Pathology landscape in US has undergone a tectonic shift with the emergence of new arrangements for anatomic pathology services. One such setup, 'In-Office Pathology Lab' (IOP) has been widely adopted by the urology community. In this study we analyze our GU pathology consultation data to assess concordance with cases received from IOP.

Design: Of the 1237 consecutive bladder and prostate biopsy cases sent to us for consultation by various IOP during 2011-2013, 1011 (140 bladder \& 871 prostate) cases that either had an accompanying outside final report (urologist request; 182) or a preliminary report with a diagnosis (pathologist request; 829) were selected. All consultations were diagnosed by a single expert GU pathologist. The overall diagnosis were compared and only the discrepancies that could potentially alter therapy or prognosis were recorded as significant discrepancies (SD). SD due to differences of Gleason score (GS) were congruent with the newly proposed Prognostic Gleason Grade Grouping (PG)

Results: Overall we agreed with $653(65 \%)$ cases. There were SD in $24 \%$ bladder and $37 \%$ prostate cases; with relatively similar distribution in urologist $(33 \%)$ versus pathologist $(36 \%)$ driven cases. However, SD that changed the cases from benign to carcinoma (CA) and vice versa were more frequent when requested by a urologist rather than pathologist $(5.5 \%$ vs $2.2 \%, \mathrm{p}<0.05)$ and more frequent in bladder as compared to prostate $(9 \%$ vs $2 \%, p<0.001)$. Ten $(13 \%)$ benign prostate cases were diagnosed as CA, most $(70 \%)$ of which were requested by urologists. We agreed with $44 \%$ of Atypical (ATYP) prostate cases whereas the other $56 \%$ were diagnosed as either benign $(19 \%)$ or CA (34\%). SD related to GS/PG were identified in 121 (24\%) CA cases (62\% upgraded and $38 \%$ downgraded). Gleason pattern 5 was missed outside in $33 \%$ of the CA cases in which we identified Gleason pattern 5 .

Correlation of Outside (O) and Expert (EX) Diagnoses of Prostate cases

\begin{tabular}{|c|c|c|c|c|}
\hline & O BENIGN (78) & O HGPIN (23) & O ATYP (241) & O CA (519) \\
\hline EX BENIGN (107) & $65 \%$ & $30 \%$ & $19 \%$ & $1 \%$ \\
\hline EX HGPIN (30) & $5 \%$ & $52 \%$ & $3 \%$ & $0 \%$ \\
\hline EX ATYP (141) & $17 \%$ & $13 \%$ & $44 \%$ & $4 \%$ \\
\hline EX CA (588) & $13 \%$ & $5 \%$ & $34 \%$ & $95 \%$ \\
\hline
\end{tabular}

5 cases had other diagnoses

Conclusions: We found high discordance in several categories. As these issues may not be limited to IOP and carry significant clinical implications, their awareness is crucial. The explanation is multifactorial. Pathologist's expertise, inter-observer variability and factors inherent to IOP seem to be the key. These warrant concerted knowledge-transfer activities and a closer look at the IOP, which may require sub-specialty proficiencybased enhancements.
1068 ERG Oncoprotein Expression in Prostate Core Biopsies: Diagnostic Utility and Correlation with the New Proposed Prognostic Gleason Grade Grouping

AT Savera. PLUS Diagnostics, Union, NJ.

Background: Diagnosis of limited prostate cancer (PC) can be challenging due to overlapping features of benign mimickers (BM) of PC. A diagnosis of 'Atypical Glands Suspicious for Cancer' (ATYP) is rendered in cases that fall short of a definitive diagnosis of PC, necessitating a repeat biopsy. The TMPRSS2-ERG gene fusion that leads to ERG overexpression is common in clinically localized PC. The anti-ERG antibody highly correlates with ERG rearrangement and can potentially aid in work up of ATYP cases. However, conflicting data exists regarding ERG's utility in classifying ATYP lesions. Likewise, the relationship between ERG and Gleason score (GS) is unclear. Design: We reviewed 346 prostate core biopsies from 339 patients. Each core was classified as Benign, HGPIN, ATYP or PC and BM were also recorded. Each core with PC was assigned a GS and Prognostic Gleason grade group (PGP). Each core was stained with ERG $(9 \mathrm{FY})$ and positivity was graded as $1=<20 \%, 2=20-60 \%$ and $3=>60 \%$. Results: Results of ERG expression are detailed in the table.

\begin{tabular}{|c|c|c|c|c|c|c|}
\hline Type & Total & ERG - & 1 & 2 & 3 & Total ERG + \\
\hline PC 3+3 (I) & 51 & 21 & 0 & 1 & 29 & $30(59 \%)$ \\
\hline PC 3+4 (II) & 29 & 12 & 1 & 1 & 14 & $16(55 \%)$ \\
\hline PC 4+3 (III) & 19 & 12 & 0 & 0 & 7 & $7(37 \%)$ \\
\hline PC 8 (IV) & 25 & 17 & 0 & 0 & 8 & $8(32 \%)$ \\
\hline $\mathrm{PC} 9 / 10(\mathrm{~V})$ & 17 & 15 & 0 & 0 & 2 & $2(12 \%)$ \\
\hline ATYP & 23 & 11 & 0 & 0 & 12 & $12(52 \%)$ \\
\hline HGPIN & 48 & 43 & 0 & 1 & 4 & $5(10 \%)$ \\
\hline BENIGN & 134 & 133 & $1 *$ & 0 & 0 & $1(0.005 \%)$ \\
\hline
\end{tabular}

I-V represent PGP. * Focal staining in low grade PIN

ERG expression was more frequent in lower GS/PGP PC and significantly correlated with GS/PGP (R2=0.9873, p=0.0017). In the $141 \mathrm{PC}$, adjacent/intermingled Benign, HGPIN and Intraductal PC were noted in 123, 31 and 4 respectively, of which 2 $(0.01 \%)$ Benign (focal low-grade PIN), 12 (39\%) HGPIN and $1(25 \%)$ Intraductal PC were ERG+; the associated PC was also ERG + . No ERG expression was identified in BM - adenosis (7), partial atrophy (42), post atrophic hyperplasia (39), proliferative inflammatory atrophy (19), simple atrophy (44), basal cell hyperplasia (8) and seminal vesicle (12). Overall ERG differential expression in $45 \%$ PC, $52 \%$ ATYP, $21 \%$ HGPIN and $0.006 \%$ Benign was statistically significant $(\mathrm{p}<0.0001)$.

Conclusions: ERG is a highly specific marker of PC. In conjunction with morphology and other immunohistochemical markers, ERG can enable a definitive diagnosis in a subset of ATYP cases. In a proper setting and excluding HGPIN related lesions, ERG expression is diagnostic of PC. ERG expression significantly correlates with GS and PGP. The inverse relationship between ERG expression and GS/PGP mitigates an association between ERG rearrangement and PC aggressiveness.

1069 Automated Gland Ring Morphometry for Prostate Cancer Gleason Scoring in Multispectral Immunofluorescent Images

$R$ Scott, FM Kahn, C Cordon-Cardo, MJ Donovan, G Fernandez. Icahn School of Medicine, New York, NY.

Background: The prostate needle biopsy (PNB) Gleason score (bGS) is the primary measure used to guide treatment for patients newly diagnosed with prostate cancer (PCa). Manual Gleason scoring of H\&E stained tissue is limited by intra and interobserver reproducibility based on subjective interpretation. Automated approaches to characterize glandular ring structures arranged around a central lumen, the cornerstone of Gleason grading, have struggled to yield robust metrics that do not rely on semiautomated segmentation methods.

Design: Utilizing 926 PNB multispectral immunofluorescent images (IF) from 306 patients, we present a new approach for segmenting gland rings (GRs) and demonstrate correlation with bGS. The segmentation algorithm enhances previously published methods for extracting labeled nuclei, cytoplasm and lumen from DAPI and CK18 biomarker images. The algorithm leverages a graph-based approach, clustering the Delaunay triangulation/Voronoi diagram of nuclear coordinates to generate polygonal areas, which are then classified into 4 regions: good rings, glandular fragments, stroma, and low-confidence/under-segmented areas. Our morphologic features are based on the observation that the breakdown of GRs tends to reduce the proportion of gland nuclei adjacent to stroma and lumens. Weighted ratio and mean features are defined over a subset of regions, eg quantifying the proportion of GRs boundaries touching stroma (ST) weighted by epithelial nuclear area (ENA) in good rings. Segmentation accuracy against manually annotated ground truth is evaluated on a randomly selected subset of 266 images, calculating sensitivity, specificity and Dice metrics for good ring areas. Correlation with bGS in the complete 926 image set was done employing the Pearson's correlation coefficient (pcorr) with p-value for significance (p).

Results: GR segmentation accuracy was excellent (sensitivity $88 \%$, specificity $96 \%$, Dice $89 \%$ ). Features had high (negative) correlation with bGS, including a measure of GR ratio area touching lumens and stroma (pcorr $-0.45, \mathrm{p}<0.0001$ ), a measure of GRs ST normalized by ENA (pcorr $-0.47, \mathrm{p}<0.0001$ ) and a ratio of ENA in good rings refined by thresholding on ST and ring diameter (pcorr $-0.48, \mathrm{p}<0.0001$ ).

Conclusions: We have developed an accurate and robust automated system for segmentation and Gleason scoring of PNB IF images. Quantitative metrics from the system may assist in PCa prognosis. 
1070 Diagnostic Criteria for Ductal Adenocarcinoma of the Prostate. Interobserver Variability among 20 Expert Uropathologists

AH Seipel, B Delahunt, H Samaratunga, M Amin, J Barton, DM Berney, A Billis, L Cheng, E Comperat, A Evans, SW Fine, D Grignon, PA Humphrey, C Magi-Galluzzi, $R$ Montironi, I Sesterhenn, JR Srigley, K Trpkov, T van der Kwast, M Varma, M Zhou, A Ahmad, S Moss, L Egevad. Karolinska Institutet, Stockholm, Sweden; University of Otago, Wellington, New Zealand; Aquesta Pathology, Brisbane, Australia; Cedars-Sinai Medical Center, Los Angeles, CA; Joint Pathology Center, Silver Spring, MD; Queen Mary University of London, London, United Kingdom; State University of Campinas, Campinas, Brazil; Indiana University School of Medicine, Indianapolis, IN; Hopital La Pitié-Salpetrière, Paris, France; University of Toronto, Toronto, Canada; Memorial Sloan-Kettering Cancer Center, New York, NY; Washington University School of Medicine, St Louis, MO; Western Reserve University, Cleveland, OH; Polytechnic University of the Marche Region, Ancona, Italy; McMaster University, Hamilton, Canada; University of Calgary, Alberta, Canada; University Hospital of Wales, Cardiff, United Kingdom; New York University Langone Medical Center, New York, NY. Background: The reported incidence of ductal adenocarcinoma of the prostate (DAC) varies widely $(0.13 \%-12.7 \%)$ in the literature. The aim of this study was to investigate the interobserver variability of the diagnosis of DAC among twenty experts in uropathology and utilize the findings to define diagnostic criteria for this tumor type.

Design: Photomicrographs of 21 carcinomas with ductal morphology were distributed in a web-based questionnaire to 20 genitourinary pathologists from 8 countries. Results: In $11(52 \%)$ cases a $2 / 3$ consensus was reached for a diagnosis of DAC and in $5(24 \%)$ there was consensus against this diagnosis. In cases of DAC the respondents reported papillary architecture $(86 \%)$, stratification of nuclei $(90 \%)$, high-grade nuclear features $(54 \%)$, tall columnar epithelium $(53 \%)$, elongated nuclei $(52 \%)$, cribriform architecture (40\%) and necrosis (7\%). The most important diagnostic feature reported for DAC was papillary architecture $(59 \%)$, while nuclear and cellular features were considered most important in only $2 \%$ to $11 \%$ of cases. The most common differential diagnoses were intraductal prostate cancer (52\%), high-grade PIN (37\%) and acinar adenocarcinoma (17\%). Reported reasons for not diagnosing DAC were lack of typical architecture $(33 \%)$, cellular morphology $(18 \%)$ or a combination of both $(9 \%)$.

Conclusions: This study has shown that papillary architecture is the most useful diagnostic feature of DAC, while nuclear and cellular features were considered less important. DAC was less likely to be diagnosed in the absence of papillary architecture and nuclear stratification.

1071 Heterogeneity of PTEN and ERG Biomarkers Expression in Prostate Cancer Needle Biopsies with More Than One Core Positive: Implications for Biomarkers Sampling Strategies

RB Shah, A DeMarzo. Miraca Life Sciences Research Institute, Irving, TX; Johns Hopkins Hospital, Baltimore, MD.

Background: ERG and PTEN biomarkers are increasingly analyzed in prostate needle biopsies (NBXs); ERG as a marker of clonality and number of tumor foci and PTEN due to its prognostic relevance.

Design: We evaluated inter- (between different tumor sites/cores) and intra-focal (within same tumor core) for ERG overexpression (C-terminus, EPR 3864, Epitomics) and PTEN loss (rabbit monoclonal antibody, cell signaling) in 194 NBX PCa cases containing $>1$ cancer cores to evaluate the pattern of immunohistochemical staining heterogeneity and its implications for sampling strategies. The Gleason score ranged from 6 to 9 and the number of cores positive ranged from 2 to 11 . All cores positive were evaluated.

Results: Of 194 PCa cases, ERG overexpression and PTEN loss in at least one cancer core was present in $111(57 \%)$ and $69(36 \%)$ cases respectively. Inter-core heterogeneity for ERG occurred in 47 (42\%), suggesting the presence of at least two separate clones in the prostate. Intra-core heterogeneity for ERG was present in $41(37 \%)$ indicating multiple clones can be present even within the same core needle biopsy/site. Intercore heterogeneity for PTEN was present in $47(68 \%)$ and intra-focal heterogeneity was $47(68 \%)$. For cases with differing Gleason score between separate tumor sites $(49 \%)$, the cancer core with the highest Gleason score was representative of ERG overexpression and PTEN loss in $84 \%$ and $92 \%$ of cases and the core with the highest tumor volume represented ERG overexpression and PTEN loss abnormality in $96 \%$ and $80 \%$ of cases respectively. Measurement of both highest Gleason score and tumor volume cores represented ERG overexpression and PTEN loss abnormality in 92\% and $98 \%$ of cases. For cases with similar Gleason score across tumor sites $(51 \%)$, the cancer core with highest tumor volume represented ERG overexpression and PTEN loss abnormality in $90 \%$ and $76 \%$ of cases. Addition of second highest tumor volume improved representation of ERG overexpression and PTEN loss in $94 \%$ and $84 \%$ of cases.

Conclusions: When multiple cores are involved by PCa, selection of at least two cores representing highest Gleason score and tumor volume provide cost-effective yet optimal representation of underlying molecular abnormality. Nevertheless, due to significant inter- and intra-focal tumor expression heterogeneity, complete assessments of the status of these markers for all positive cores may be appropriate in cases where both selected cores lack molecular abnormality.

\section{Uroplakin II Is a Useful Immunomarker for Identification of} Urothelial Carcinomas

A Shahab, H Liu, F Lin. Geisinger Medical Center, Danville, PA.

Background: Uroplakins are a group of transmembrane proteins which are specific products of urothelial cells. Among uroplakins, uroplakin III (UP III) has been demonstrated to be a useful marker in identifying urothelial tumors. Although UP III is quite specific, the sensitivity is very low (around $30 \%$ ). On the contrary, one recent study reported that uroplakin II (UP II), a recently described immunomarker of the uroplakin family, had a superior sensitivity of $78 \%$ in identification of urothelial tumors of all grades combined when compared to UP III. However, the diagnostic specificity of UP II has not been extensively tested. In this study, we further investigated the diagnostic sensitivity and specificity of UP II in a large series of urothelial neoplasms, non-urothelial tumors and normal tissues.

Design: Immunohistochemical evaluation of UP II (BC21, Biocare) expression was performed on 123 urothelial neoplasms, 1845 non-urothelial tumors, and 310 normal tissues from various organs on tissue microarray sections. The staining intensity was graded as weak or strong. The staining distribution was interpreted as negative $(<5 \%$ tumor cells stained), $1+(5-25 \%), 2+(26-50 \%), 3+(51-75 \%)$, and $4+(>75 \%)$

Results: The staining results for urothelial neoplasms are summarized in Table 1. Overall, 86 of $123(69.9 \%)$ urothelial neoplasms were positive for UP II, with a diagnostic sensitivity of $88.5 \%, 80.8 \%$, and $41.4 \%$ in low-grade (LG) non-invasive urothelial carcinoma (UC), high-grade (HG) non-invasive UC, and invasive UC, respectively. All non-urothelial tumors and normal tissues were negative for UP II.

\begin{tabular}{|c|c|c|c|c|c|c|}
\hline \begin{tabular}{|l|} 
Urothelial \\
Neoplasms \\
\end{tabular} & Negative & $1+$ & $2+$ & $3+$ & $4+$ & $\begin{array}{l}\text { Total Positive Case } \\
(\%)\end{array}$ \\
\hline $\begin{array}{l}\text { LG Non-invasive } \\
\text { UC }(\mathrm{N}=35\end{array}$ & $\| 4(11.4 \%)$ & $9(35.7 \%)$ & $9(35.7 \%)$ & $7(30 \%)$ & $6(17.1 \%)$ & $31(88.5 \%)$ \\
\hline $\begin{array}{l}\text { HG Non-invasive } \\
\text { UC (N=47) }\end{array}$ & $9(19.1 \%)$ & $\begin{array}{l}17 \\
(36.1 \%)\end{array}$ & $6(12.7 \%)$ & $7(14.8 \%)$ & $8(17 \%)$ & $38(80.8 \%)$ \\
\hline Invasive UC $(\mathrm{N}=41)$ & $24(58.5 \%)$ & $4(9.7 \%)$ & $3(7.3 \%)$ & $2(4.8 \%)$ & $8(19.5 \%)$ & $17(41.4 \%)$ \\
\hline
\end{tabular}

Conclusions: Our study demonstrated that UP II is a highly specific and relatively sensitive immunomarker for the identification of urothelial origin when working on a tumor of uncertain origin. Additionally, UP II may play a crucial role in regulating tumor progression because of the marked reduction of UP II expression from LG noninvasive UC to invasive UC.

\section{Clinicopathologic Features of Renal Cell Carcinoma in a} Contemporary Partial Nephrectomy Series

SS Shen, LD Truong, JY Ro, AG Ayala. Houston Methodist Hospital, Houston, TX. Background: Nephron sparing partial nephrectomy is increasingly used for treatment of solitary renal masses during last decade due to advances of surgical technique and realization of the importance of preservation of renal function for long term survival. The indications for this procedure continue to expand over the recent years. We reviewed the clinicopathologic features of a large series of partial nephrectomy highlighting high risk features.

Design: A total of 560 patients with partial nephrectomy specimens for renal mass from a single institution (2003 to 2013) were reviewed and those with renal cell carcinoma (RCC) were selected for further study. Evaluated clinicopathologic features included patients' age, gender, and laterality, and tumor characteristics including tumor size, grade, stage, vascular invasion and margin status. The margin status was correlated with other pathologic features.

Results: Of the 560 patients underwent partial nephrectomies, 439 (78.6\%) had renal cell carcinoma (RCC). The average patient's age was 58.9 years (range 17.7-92.5). The male to female ratio was $1.5: 1$. The average tumor size was $3.1 \mathrm{~cm}$ (range 0.2 to 8.5 $\mathrm{cm})$. Three hundred and forty seven $(79 \%), 87(19.8 \%)$, and $5(1.1 \%)$ tumors were $\leq 4$ $\mathrm{cm},>4$ but $\leq 7 \mathrm{~cm}$, and $>7 \mathrm{~cm}$, respectively. The most common histologic types were clear cell RCC $(\mathrm{n}=319,72.5 \%)$, papillary RCC $(\mathrm{n}=84,19.1 \%)$, chromophobe RCC $(n=25,5.7 \%)$, clear cell papillary RCC $(n=7,1.6 \%)$. In addition, there were 2 cases of tubulocystic RCC and 1 case each of mucinous tubular spindle cell RCC and unclassified RCC. Three hundred and forty $(77.3 \%)$ and $81(18.4 \%)$ were pT1a and pT1b tumors, respectively. Two were pT2 tumors, and 13 cases $(3 \%)$ had perirenal fat invasion (pT3a). One hundred forty five (33\%) of the tumor was Fuhrman nuclear grade 3 or 4 and 2 cases of clear cell RCC had sarcomatoid changes. Four cases had lymphovascular invasion. Sixteen cases had positive parenchymal margins. The positive margin was not correlated with tumor size, pT stage, histologic type or histologic grade of tumor. Conclusions: In this contemporary series of partial nephrectomy, we have shown that there is significant number of tumors which have high risk features such as large tumor size, perirenal fat invasion, high Fuhrman nuclear grade and positive surgical margins. Long-term clinical follow up is necessary to further stratify the risk group and appropriate selection for partial nephrectomy.

1074 Not All Gleason Pattern 4 Prostate Cancers Are Created Equal: A Study of Two Autopsy Series

F Siadat, D Pushkar, S Egawa, NE Fleshner, N Aldaoud, AR Zlotta, $T$ Van der Kwast. University Health Network, Toronto, ON, Canada; University of Moscow, Moscow, Russian Federation; Jikei University School of Medicine, Tokyo, Japan; Jordan University of Science and Technology, Irbid, Jordan.

Background: In prostate cancers (PC) Gleason pattern 4 (G4) has considerable morphological variability and some studies suggest that different G4 patterns have different clinical outcome. We hypothesized that the prevalence of clinically significant G4 patterns in autopsy prostates should not exceed the $4 \%$ life-time risk of prostate cancer death. We studied the prevalence of G4 patterns in two populations, not exposed to PSA testing, to examine their correlation with known poor prognostic factors.

Design: Between 2008-2011, we collected prospectively 320 autopsy prostate specimens, including 100 Asian men from Japan (ASI) and 220 Caucasian from Russia (CAU). None of the men had been diagnosed with PC before their death. Prostates were removed en-bloc with seminal vesicles within $24 \mathrm{~h}$ after death. They were sectioned and embedded in toto. H\&E slides were examined at Toronto General Hospital and in those with PC, Gleason score (GS), various G4 patterns, including intraductal carcinoma (IDC), tumour volume and extra-prostatic extension (EPE) were recorded. The likelihood ratio (LR) was calculated for G4 patterns against EPE. 
Results: Mean age was 64.4 y (ASI: 68.5 , CAU: 62.6 ), $36.6 \%$ had PC (ASI: $35 \%$, CAU: $37.3 \%$ ) with mean tumour volume of $0.30 \mathrm{~cm}^{3}$ (ASI: $0.42 \mathrm{~cm}^{3}$, CAU: $0.25 \mathrm{~cm}^{3}$ ). $25 \%$ of autopsy prostates had PC GS6 or lower. $11.6 \%$ had a GS 7 or higher. GS $\geq 7$ PC was more prevalent in the ASI group (p: 0.02). G4 patterns included: small fused glands ( $\mathrm{SFG}, 8.4 \%$ ), poorly formed glands ( $\mathrm{PFG}, 4.4 \%$ ), small cribriform (SC, $2.2 \%$ ), intraductal carcinoma (IDC, $2.2 \%$ ), large cribriform (LC, $1.9 \%$ ), and glomeruloid pattern (GP, $0.6 \%$ ). Tumour volume was larger in presence of IDC or LC as compared to other $\mathrm{G} 4$ patterns $\left(1.28 \mathrm{vs} 0.22 \mathrm{~cm}^{3}, \mathrm{p}<0.001\right)$. EPE was observed in $4.4 \%$ of cases (no difference between ASI and CAU). LC and IDC were strongly correlated with EPE (LR: 17.8 and 15, $\mathrm{p}<0.001$, respectively) followed by SC pattern (LR: 9.1, p: 0.003). In contrast, LR for EPE was 3.31 (p: 0.07) in PFG and 1.31 (p: 0.25) in SFG.

Conclusions: $\mathrm{LC}$ and IDC are strongly correlated with poor outcome features and have a lower prevalence than the expected mortality from PC. These patterns may behave more aggressively than the other $\mathrm{G} 4$ patterns.

1075 The Epithelioid and Sarcomatoid Components of Sarcomatoid RCC Are Molecularly Similar to Each Other but Distinct from Fuhrman Grade 4 Non-Sarcomatoid RCC

$K$ Sircar, S-Y Yoo, T Majewski, L Patel, H Voicu, K Wani, P Tamboli, K Baggerly, $K$ Aldape, B Czerniak. UT MD Anderson Cancer Center, Houston, TX; Baylor College of Medicine, Houston, TX

Background: Sarcomatoid clear cell RCC (sRCC), though categorized as grade 4 using the Fuhrman grading system, shows a worse cancer specific mortality compared to non-sarcomatoid grade 4 RCC. A molecular understanding of sRCC has been limited due, in part, to the absence of genome wide copy number and transcript examination. Hence, we sought to molecularly characterize sarcomatoid change in clear cell RCC at the copy number and transcript levels and to compare our results with advanced non-sarcomatoid clear cell RCC (nsRCC).

Design: DNA copy number was assessed using the Illumina HumanOmniExFFPE platform for which FFPE samples from 9 patients with $\mathrm{sRCC}$ (stage 4, $\mathrm{n}=7$; stage 3, $\mathrm{n}=2$ ) were macrodissected for sarcomatoid (S) and epithelioid (E) areas. As controls, we used TCGA copy number data from 158 patients with nsRCC of advanced stage (stage 4, $n=57$; stage 3, n=101) and Fuhrman grade 4 nsRCC (stage 4, n=15; stage 3, n=4). The transcriptome was examined by expression microarray (Illumina HumanRef-8) using FFPE samples from 17 patients with $\operatorname{sRCC}$ (stage $4, n=14$; stage $3, n=2$; stage $2, n=1$ ) and from 8 patients with grade 4 nsRCC (stage $4, n=6$; stage $3, n=2$ ). Subsequently, RNA-Seq (Illumina HiSeq) was performed on independent patient samples: 7 sRCC (stage $4, n=6$, stage $3, n=1$ ) and 6 grade 4 nsRCC ( stage $4, n=5$; stage $3, n=1$ )

Results: Multiple copy number and transcriptomic aberrations were seen in sRCC, including known poor prognostic changes ( $9 p / 14 q$ loss, ccB expression profile). Copy number and transcriptomic differences between the E and S components of sRCC were negligible. When comparing sRCC $(\mathrm{E} / \mathrm{S})$ to nsRCC, however, many genes were significantly associated with copy number variations $(1079$ genes, FDR $<0.05$ ) Subset analysis of Fuhrman grade $4 \mathrm{nsRCC}$ also showed significant differences when compared to sRCC, both in terms of copy number $(347$ genes, FDR $<0.05)$ and transcript abundance $(1396$ genes, FDR $<0.05)$. RNA-seq confirmed the transcriptomic similarity of $\mathrm{E}$ and $\mathrm{S}$ components and their distinctiveness from Fuhrman grade 4 nsRCC using independent samples $(\mathrm{n}=659$ genes, $\mathrm{FDR}<0.05$ ).

Conclusions: The discrete DNA copy number and gene expression signatures of sarcomatoid RCC suggests that it should be categorized separately from non-sarcomatoid Fuhrman grade 4 RCC. Despite stark morphologic differences, the E and S components of sarcomatoid RCC are molecularly similar suggesting that its genetic programming is largely embedded in its epithelioid component.

1076 Metanephric Adenoma: The Utility of Immunohistochemical and Cytogenetic Analyses in Differential Diagnosis

SN Slemp, O Hes, SR Williamson, JN Eble, DJ Grignon, M Wang, S Zhang, LA Baldrige G Martignoni, M Brunelli, L Wang, E Comperat, GT MacLennan, L Cheng. Indiana University School of Medicine, Indianapolis, IN; Charles University Hospital Plzen, Pilsen, Czech Republic; Henry Ford Health System, Detroit, MI; Universitá di Verona, Verona, Italy; Groupe Hospitalier Pitié-Salpêtrière, Paris, France; Case Western Reserve University, Cleveland, $\mathrm{OH}$

Background: Metanephric adenoma is a benign renal neoplasm that overlaps in morphology with papillary renal cell carcinoma and epithelial-predominant nephroblastoma. To aid in resolving this differential diagnosis, we investigated the utility of immunohistochemical (IHC) and molecular analyses in distinguishing between these entities.

Design: We analyzed 36 tumors originally diagnosed as metanephric adenoma using a combination of IHC and fluorescence in situ hybridization (FISH). IHC staining was performed for cytokeratin 7 (CK7), $\alpha$-methylacyl-CoA-racemase (AMACR), WT1, and CD57. Staining pattern, intensity, and extent were evaluated using a four-tiered (0-3) system. The combination of CK7-, AMACR-, WT1+, CD57+ was considered characteristic of metanephric adenoma. FISH was performed on a subgroup of cases that deviated from this staining pattern or showed unusual histologic features to identify abnormalities of chromosomes 7, 17, and Y.

Results: Most tumors showed the expected staining pattern of metanephric adenoma: CK7- (34/36), AMACR- (33/36), WT1+ (31/36), and CD57+ (35/36). Of tumors that deviated from this staining pattern, one yielded the opposite results (CK7+, AMACR+, WT1-, CD57-) and another was positive for CK7, AMACR and CD57 but negative for WT1. A third tumor was positive for WT1, CD57, and AMACR and negative for CK7. Two tumors showed membranous rather than cytoplasmic positivity for CD57. Focal, limited reactivity for AMACR and CK 7 was present in 3 tumors and 4 tumors, respectively, interpreted as negative. Of the tumors with discordant IHC staining patterns, the first (CK7+, AMACR+, WT1-, CD57-) exhibited trisomy of chromosomes
7 and 17 and loss of Y by FISH. The second (CK7+, AMACR+, CD57+, WT1-) demonstrated loss of Y only. Based on these IHC and molecular profiles, these two tumors were reclassified as papillary renal cell carcinomas. The remaining tumors with equivocal IHC features demonstrated disomy of chromosomes 7, 17, and Y, supporting the original classification of metanephric adenoma

Conclusions: Our results support the clinical application of immunohistochemical staining and FISH in establishing the diagnosis of metanephric adenoma, particularly in cases with borderline morphologic or immunohistochemical features.

\section{Smooth Muscle and Renal Tubulopapillary Epithelial Tumor} (SMARTPET): A Distinctive Biphasic Renal Neoplasm

NE Smith, GJ Netto, PB Illei, JI Epstein, AV Parwani, ME Allaf, P Argani. The Johns Hopkins Medical Institutions, Baltimore, MD; University of Pittsburgh Medical Center, Pittsburgh, PA

Background: A number of renal neoplasms feature smooth muscle stroma and an epithelial component. We have encountered 5 cases of a distinct biphasic renal neoplasm featuring smooth muscle stroma and nodules of tubulopapillary epithelium which do not fit any accepted category.

Design: Three cases were consults to our institution and two were resected at our hospital. The clinical, immunohistochemical, and molecular features of these neoplasms were assessed.

Results: The cases included 4 females and 1 male (ages 27-70 years). Two of the cases were initially classified as mixed epithelial stromal tumor (MEST), although none had a history of exogenous hormone exposure (including the male). All four neoplasms with available history were discovered incidentally on imaging studies with sizes ranging from 4-20 cm. Histologically, they featured smooth muscle stroma alternating with looser, edematous areas, and a complex epithelial component featuring tubulopapillary nodules, branching tubules, clefts, and large cysts. None of the cases demonstrated ovarian-like stroma. The epithelium was cytologically bland and typically cuboidal and basophilic with focal areas of clear or eosinophilic cytoplasm. In 4 of 5 cases, the epithelium focally formed areas resembling nephrogenic adenoma, and only one case focally demonstrated subnuclear vacuoles. The stroma of all cases labeled diffusely for desmin. Estrogen receptor labeling was absent in 3 cases with focal $(<10 \%)$ weak labeling in the remaining two. The epithelial component of each case labeled diffusely for CK 7 and was patchy for racemase. Carbonic anhydrase IX, HMB45, and inhibin were negative in all cases. Despite the resemblance of the nodular epithelial component to low grade papillary renal cell carcinoma, none the 4 tested cases demonstrated trisomies of chromosome 7 or 17 by FISH. One case sent for cytogenetics demonstrated a complex karyotype with multiple chromosome translocations. Two patients with significant follow-up are disease free at 18.5 and 2.5 years.

Conclusions: SMARTPET could potentially represent a variant of MEST with smooth muscle stroma and tubulopapillary epithelial proliferation. However, the absence of clinical history of hormone exposure, minimal to no labeling for estrogen receptor, lack of ovarian stroma, and extent of epithelial proliferation suggests a distinct entity. Separation of SMARTPET from MEST may make the latter a more homogeneous entity. We suggest close follow-up for these lesions as their biologic behavior is not clear.

1078 Neuroendocrine Differentiation (NED) in Prostatic Adenocarcinoma (PCa): A Dual-Institutional Review with Respects to a Newly Proposed Classification System

SC Smith, SK Mohanty, AS McDaniel, MN dePeralta-Venturina, DJ Luthringer, SA Tomlins, MB Amin. Cedars-Sinai Medical Center, Los Angeles, CA; University of Michigan, Ann Arbor, MI.

Background: Recent advances in urologic oncology have resulted in increased saliency of recognition of NED in $\mathrm{PCa}$, prompting development of a proposed classification system sponsored by the Prostate Cancer Foundation (PCF) to standardize diagnostic terminology. To better understand the spectrum, prevalence, and clinical features of lesions encountered, we reviewed the experience of two academic centers with respects to this new classification.

Design: From one institution we reviewed all lesions diagnosed as PCa with focal or greater NED as supported by prospective immunohistochemistry (IHC) from 1994-present $(n=34)$; consultation cases were included $(n=7)$. From the second institution, additional neuroendocrine cases including metastatic lesions $(n=10)$ and rapid autopsy cases $(n=3)$ were reviewed to subtend a full range of lesions with NED. Lesions were classified by the proposed PCF system criteria. Immunostains were performed for IHC markers of NED and prostatic differentiation.

Results: Of all primary cases reviewed $(n=46)$, we observed that $14 \%$ were usual $\mathrm{PCa}$ with NED, $5 \%$ were PCa with Paneth Cell-like NED, $42 \%$ were small cell ( $26 \%$ pure, $16 \%$ mixed with usual PCa), and $2 \%$ were large cell neuroendocrine carcinoma. Two additional groups of $\mathrm{PCa}$ were identified. One group showing hybrid features and corresponding to a provisional group in the PCF system termed "PCa with overlap features of small cell and usual $\mathrm{PCa}$ " included up to $28 \%$ of cases. Four other lesions were $\mathrm{PCa}$ with a striking carcinoid-tumor like morphology and immunoprofile mixed with PCa (thus not meeting PCF carcinoid tumor criteria). Three cases demonstrated "small cell-like change". NED marker prevalence was synaptophysin (93\%), chromogranin (69\%), and CD56 (63\%). CD44, a putative NE and stem cell marker, was positive in $60 \%$ of cases, including both small cell and non-small cell cases. Histogenesis markers positive in the NE component included NKX3.1 (50\%), p501S (41\%), and PSMA (53\%), with negative cases typically small cell carcinoma. ERG was positive in $21 \%$ of cases. 
Conclusions: NED exhibits a broad morphologic spectrum in PCa. The proposed PCF classification was successfully applied in this review. Infrequent subsets of cases show features overlapping between these categories or PCa with foci of carcinoid-like morphology; these require further study to ascertain their biologic potential and place in the classification system.

1079 Uroplakin II Outperforms Uroplakin III in Diagnostically Challenging Settings

SC Smith, SMohanty, LP Kunju, E Chang, F Chung, JC Carvalho, GP Paner, DE Hansel, $D J$ Luthringer, MN dePeralta-Ventrurina, MB Amin. Cedars-Sinai Medical Center, Los Angeles, CA; University of Michigan, Ann Arbor, MI; University of Chicago, Chicago, IL; University of California, San Diego, San Diego, CA.

Background: Biomarkers for distinguishing high grade urothelial carcinoma (UC) from other poorly differentiated carcinomas suffer from lack of specificity (p63, HMWCK, CK7/20, etc), including novel urothelial-associated markers (GATA3, S100P). Uroplakin III (UP3), a 47kDa transmembrane constitutent of urothelial plaques, is known to be the most specific marker, though it lacks sensitivity. A newly developed antibody against uroplakin II (UP2), a different 15kDa plaque protein, appears to have superior sensitivity and similar specificity to UP3 in studies of primary tumors. Direct comparisons have not been made in the diagnostically challenging settings of 1) high grade UC $v s$ prostate $(\mathrm{CaP})$ in the bladder neck, 2) high grade UC vs renal cell carcinoma variants in the upper tract, and 3) metastases, where accurate classification implies therapeutic ramifications. Design: Whole sections of high grade bladder neck lesions $(\mathrm{n}=35, \mathrm{UC}$ and $\mathrm{CaP})$ and metastatic UC $(n=30)$ and a tissue microarray (TMA) of upper tract urothelial $(n=19)$ and high grade renal $(\mathrm{n}=51)$ carcinomas were stained by automated immunohistochemistry with UP3 (clone BC17) and new UP2 (clone BC21). Additional TMAs of a wide variety of other cancers $(n=98)$ were also stained. Expression was scored from 0-3 based on intensity and proportion of cells positive, with analysis by Wilcoxon matched pairs tests and McNemar tests.

Results: UP3 showed membranous "plaque-like" stain, while UP2 showed both membranous and cytoplasmic positivity. Significantly greater intensity $(p=0.003)$ and proportion $(\mathrm{p}=0.03$ ) of positive staining was noted for UP2 as compared to UP3 in bladder neck lesions, consistent with this test showing greater sensitivity $(63 \% \mathrm{vs}$ $30 \%)$ and similar specificity $(95 \%$ vs $100 \%)$ for UC, $\mathrm{p}=0.004$. Among upper tract lesions, UP2 showed greater intensity and proportion than UP3, (both, $\mathrm{p}=0.02$ ), consistent with a trend toward greater sensitivity $(47 \%$ vs $14 \%)$ and equal specificity (both $100 \%$ ) for UC, $\mathrm{p}=0.06$. Among UC metastases, UP2 showed greater intensity and proportion than UP3, (both $\mathrm{p}<0.001$ ), consistent with higher sensitivity $(73 \% \mathrm{vs}$ $37 \%$, respectively, $\mathrm{p}=0.001$ ). Of 98 additional cancer cases tested, no non-urothelial cases stained positive for either UP.

Conclusions: The UP2 antibody monotonically outperforms UP3. UP2 is a useful addition to the diagnostic armamentarium to prove urothelial histogenesis in the workup of poorly differentiated carcinomas.

1080 Pseudohyperplastic Perineural Invasion (PNI) in Prostatic Adenocarcinoma (PCa.): A Mimicker of Benign Hyperplastic Glands and HGPIN

JS So, JI Epstein. St. Luke's Medical Center, Quezon City, Philippines; The Johns Hopkins Hospital, Baltimore, MD.

Background: PNI on biopsy has both diagnostic and prognostic implications in PCa. On biopsy, PNI is one of four pathognomonic features of PCa. and is associated with an increase risk of extra-prostatic extension, where its finding can affect therapy.

Design: From 1/1/13-6/30/13, 3,120 cases of PCa. were seen by our Genitourinary Pathology Consultation Service.

Results: Of these 3,120 cases, $418(13.4 \%)$ had PNI. During this interval, we prospectively identified an unusual pattern of PNI which we have termed "pseudohyperplastic PNI" consisting of PCa. with a "gland-within-gland" morphology, where the centrally located gland wrapped around a nerve. Pseudohyperplastic PNI was found in $9(2.1 \%)$ cases with an additional 3 cores of from 2 patients biopsied at our institution with this finding also included. Of the 12 cores with pseudohyperplastic PNI, the Gleason scores were 6 in 11 cores and $4+3=7$ in the remaining core. In 6 cases, the only focus of PNI in the entire case was pseudohyperplastic. In 7 of the 12 foci, the central gland wrapping around the nerve appeared to "float" unattached within the surrounding gland closely resembling a benign hyperplastic gland or HGPIN. In the remaining 5 foci, the central PNI was focally attached to the outer gland. In 11 of 12 foci, there was no to mild cytological atypia. One focus of pseudohyperplastic PNI had prominent nucleoli in a large gland with tufting architecture and foamy cytoplasm. Of the 9 consults cases, pseudohyperplastic PNI was missed in 5 and in all 5 cases PNI was initially not diagnosed in the entire case. In 2 of these cases with missed pseudohyperplastic, PCa. was not diagnosed at the outside institution. In one of the cases biopsied at our institution, pseudohyperplastic PNI was misdiagnosed as HGPIN. Conclusions: In addition to pseudohyperplastic PNI's architecture resembling a benign gland or HGPIN, other features that contributed to making it difficult to recognize as cancer were: 1) lack of adjacent cancer in about one-half of the foci; 2) larger glands than typical cancer surrounding the PNI in a minority of cases; 3 ) tufting of the gland surrounding the PNI in a few cases; 4) atrophic or foamy gland features in some cases; and 5) most cases lacked prominent cytological atypia. While this pattern of PNI that mimics either a benign hyperplastic gland or HGPIN is uncommon, accurately recognizing it as carcinoma can have both diagnostic and prognostic implications.
1081 Prostatic Adenocarcinoma (PCa.) with Paneth Cell-Like Neuroendocrine Differentiation: Pauci-Granular Cases Potentially Misdiagnosed as Gleason Pattern 5 Cancer

JS So, JI Epstein. St. Luke's Medical Center, Quezon City, Philippines; Johns Hopkins Hospital, Baltimore, MD.

Background: Paneth cell-like change in PCa. typically has numerous eosinophilic cytoplasmic granules and is easy to diagnose.

Design: 82 cases of PCa. with Paneth cell-like granules were identified from our consultation service (2002-2013). 12 needle biopsy cases had sparse to no Paneth celllike granules and using the Gleason grading system for the Paneth cell-like component would have resulted in an assignment of Gleason pattern 5 as either the primary or secondary pattern or both.

Results: In 3 cases, there was history of prior irradiation. 11/12 cases were entirely composed of the Paneth cell-like component while the exception showed solid nests of cells with Paneth cell-like features occupying $30 \%$ of the cancer with the remaining $70 \%$ of the cancer consisting of usual Gleason score $3+3=6$ without Paneth cell-like granules. In $10 / 12$ cases, tumor occupied $\leq 10 \%$ of the core. Architectural patterns identified included: $n$ est and cords $(n=4,33 \%)$; nests only $(n=7,58 \%)$, and cords only $(n=1,9 \%)$. All 12 cases had amphophilic cytoplasm. Among the 12 cases, 7 had rare granules, 1 case had $10 \%$ of the cells with granules, and 4 cases had no granules. Within the Paneth cell-like component, rare nucleolar prominence was seen in $4 / 12(33 \%)$ cases. Of the 4 cases without any granules, all were composed of nests of cells with amphophilic cytoplasm with either no prominent nucleoli in 3 cases or rare visible nucleoli in 1 case; they would have been assigned a Gleason score 10 in 3 cases and Gleason score $5+4=9$ in the 4 th case. 8 of the cases were diffusely positive for chromogranin and synaptophysin, 2 for chromogranin only and 1 for synaptophysin only. In two cases, ki67 was performed with both showing a very low rate of $<5 \%$.

Conclusions: The key to recognizing these cases is first to note the architectural pattern of nests and cords in a small focus. Secondly, these tumors have deeply amphophilic cytoplasm with careful search in most cases revealing rare Paneth cell-like eosinophilic granules. Finally, the above finding in combination with either no prominent nucleoli or rare visible nucleoli should prompt immunohistochemical staining for neuroendocrine markers. Although further study on their prognosis is needed, currently we are not assigning a Gleason score to these cases. Their limited nature, bland cytology, and in the fews cases studied low ki67, suggest that they may not behave like Gleason pattern 5 cancer.

\section{Combination of Multiple Markers Predicts Prostate Cancer Outcome}

MA Svensson, M Nowak, W Vogel, J Carlsson, O Andren, S Perner. University Hospital of Örebro, Örebro, Sweden; University of Örebro, Örebro, Sweden; Institute of Pathology, University Hospital Bonn, Bonn, Germany.

Background: Today we are facing a large problem of overtreatment in men with prostate cancer $(\mathrm{PCa})$ because there are no good prognostic biomarkers that can help distinguish indolent from aggressive disease. Since the introduction of prostate specific antigen (PSA) testing, the lifetime risk of being diagnosed with PCa increased almost two-fold between the years 1985-2007. A large portion of PCa identified through PSA testing will never progress into a clinically relevant $\mathrm{PCa}$. Hence, there is an urgent need for reliable prognostic markers that can be used at time of diagnosis. The aim of this study is to analyze the protein expression of three genes; ERG, AR and EZH2, and the deletion status of the PTEN gene. We aim to investigate if any of these genes, alone or in combination, correlate and can predict clinical outcome.

Design: The cohort consists of 220 men that have undergone radical prostatectomy at the University Hospital of Örebro in Sweden between the years 1989-2005. Immunohistochemistry was used to detect AR, ERG and EZH2 antigen and PTEN deletion was assessd using chromogenic in situ hybridization on the DISCOVERY XT automated platform (Ventana). Protein expression was graded as negative, weak, moderate or strong. PTEN deletion was assessed as absent or present by homo- or heterozygous deletion.

Results: Our preliminary results show that ERG expression was significantly associated with time to castration resistant PCa (CRPC) and PSA-relapse, HR: 1.955 (95\% CI; 1.092-3.500) and HR: 1.454 (95\% CI; 1.073-1.970), respectively. PTEN deletion was significantly associated with time to CRPC and to PCa specific death, HR: 2.369 (95\% CI; 1.138-4.933) and HR: 3.297 (95\% CI; 1.426-7.625), respectively. There was a significant association between the combined ERG and EZH2 expression and PTEN deletion with PCa specific death. This significant association was also seen in the group of cases that harbored both ERG expression and PTEN deletion. The overall frequency showed AR, ERG and EZH2 expression in $97 \%, 44 \%$, and $73 \%$ respectively. PTEN deletion was seen in $29 \%$ of the cases, where homozygous and heterozygous deletion was present in $13 \%$ and $16 \%$, respectively.

Conclusions: The preliminary results of this study show the potential benefit of using multiple markers to predict PCa outcome.

1083 Does Clear Cell Papillary Renal Cell Carcinoma (CCPRCC) Deserve a Carcinoma Designation? A Study of 45 Patients with CCPRCC with Follow Up

V Swamy, SR Williamson, NS Gupta. Henry Ford Hospital, Detroit, MI.

Background: CCPRCC is a recently recognized distinct neoplasm with low grade morphology and unique immunohistochemical features. The aim of our study was to assess the setting in which these neoplasms arise, their possible precursor lesions, relationships to other neoplasms, and tumor behavior.

Design: All cases of renal cell carcinoma from the past 8 years were retrieved ( 2006 to 2013) to identify CCPRCC. Clinical information regarding age, sex, maximum tumor 
dimension, tumor stage, resection type and end-stage renal disease (ESRD) status were recorded. Morphologic parameters including percentages of cystic, tubular and papillary components were documented. Prognostic parameters included extrarenal extension, vascular invasion, recurrence and metastasis.

Results: During this period, a total of 980 kidney tumor specimens were diagnosed at our institution, of which 46 were CCPRCC (incidence of 4.7\%). 30/46 tumors occurred in males $(65 \%) .20$ patients had tumors in the setting of ESRD, 7 of which were multifocal. 26 patients had tumors without ESRD, 9 of which were multifocal. $26 / 46$ were partial nephrectomies (57\%). Tumor size ranged from $0.5-12.8 \mathrm{~cm} .41 / 46$ tumors $(89 \%)$ were pT1a, $4 / 46$ were pT1b and 1 tumor was pT $2.46 \%$ of tumors were predominantly cystic. All tumors showed some degree of tubular architecture. Branching tubules with intervening stroma (contrasting to back-to-back tubules) and smaller cysts with broad-based papillae were the most diagnostic features noted in CCPRCC. CK7 was positive and racemase (AMACR) was negative in all cases. No vascular invasion, extrarenal spread, recurrence or metastasis was seen in any case. Fuhrman nuclear grade was 1 in $46 \%$ and 2 in $54 \%$ of the tumors.

Conclusions: CCPRCC are indolent, low grade neoplasms that present at low stage and show predominantly cystic and branching tubular architecture. None of the tumors in this study showed aggressive features, raising the possibility that they are benign or at worst have a low malignant potential.

1084 Incidence and Clinicopathological Characteristics of Gleason Pattern 5 Prostate Cancer in Contemporary Needle Biopsies Series: A Prospective Study

$Y$ Tadros, RB Shah. Miraca Life Sciences Research Institute, Irving, TX.

Background: The presence of a Gleason pattern 5 (GP5) prostate adenocarcinoma (PCa) is associated with a worse outcome. Incidence, pattern distribution, and pathological variables associated with GP5 PCa in needle biopsies (NBXs) setting, especially after introduction of 2005 International Society of Urologic Pathologists (ISUP) modified Gleason grading system has not been addressed systematically.

Design: We evaluated 1557 consecutive and prospectively collected NBX cases to determine incidence and clinicopathological presentations of GP5 PCas. All GP5 cases were prospectively re-reviewed in the departmental consensus conference by study authors.

Results: Of 1557 NBXs, GP5 was present in $94(6 \%)$ cases. Of 664 PCa cases, GP5 represented $14 \%$ of cases. The mean age was 71 years (range: $47-92$ ), and the mean and median serum PSA values were 39 and 63 (range: $2.5-1469 \mathrm{ng} / \mathrm{ml}$ ). Mean values for percent positive cores and the core with highest tumor volume were $68 \%$ (range: $6-100 \%$ ) and $60 \%$ (range: $5-100 \%$ ) respectively. Perineural invasion and extraprostatic extension were noted in $65(69 \%)$ and $12(13 \%)$ of cases. The GP5 distribution pattern was as follows: primary $(n=2,2 \%)$, secondary $(n=30,32 \%)$, and tertiary $(n=62,66 \%)$. The architectural patterns of pattern 5 were as follows: single cells $(\mathrm{n}=71,76 \%)$, cords $(n=90,96 \%)$, sheets $(n=27,29 \%)$, and comedonecrosis $(n=2,2 \%)$. Mixture of architectural patterns specifically single cells and cords was common (78\%). Intraductal spread of invasive PCa was demonstrated in $29(31 \%)$ of GP5 cases.

Conclusions: Our results indicate GP5 is a relatively common presentation in contemporary NBX practice setting and is associated with several high risk clinicopathological features. Due to the important prognostic and therapeutic implications of GP5, pathologists must be aware of its varied patterns, clinicopathological spectrum and to the fact that the majority of GP5 cases represent a tertiary or secondary component of carcinoma.

1085 Prognostic Significance of KDM6A Protein Expression in Prostatic Adenocarcinomas (PACs)

AJ Tarasen, JV Rand, BVS Kallakury, GM Sheehan, CE Sheehan, HA Fisher, RP Kaufman, TX Nazeer, JS Ross. Albany Medical College, Albany, NY; Georgetown University Hospital, Washington, DC

Background: KDM6A/UTX is a member of a family of demethylating enzymes that remove methyl groups from nucleic acids and is thought to be a tumor suppressor by virtue of its epigenetic regulation of transcription. The KDM6A gene has been found to be mutated in a variety of solid tumors including $2 \%$ of PAC (COSMIC). In this study we investigate the expression of KDM6A in both benign prostate tissue and PAC by immunohistochemistry to assess its potential prognostic significance.

Design: Formalin-fixed paraffin-embedded tissue sections from 125 PACs were immunostained by a manual method (DAKO LSAB+ System-HRP) using rabbit polyclonal KDM6A (Thermo Pierce, Waltham, MA). Cytoplasmic and/or nuclear immunoreactivity was scored based on intensity and percentage of positive cells in both the tumor $(\mathrm{T})$ and adjacent benign (B) epithelium in each case. Cases were assessed as tumor $>$ benign $(\mathrm{T}>\mathrm{B})$, tumor=benign $(\mathrm{T}=\mathrm{B})$, tumor $<$ benign $(\mathrm{T}<\mathrm{B})$, and negative $(\mathrm{N})$. Results were correlated with clinicopathologic variables.

Results: Variable cytoplasmic KDM6A (cKDM6A) immunoreactivity was noted for both the tumor and adjacent benign glands in all cases as follows: $\mathrm{T}>\mathrm{B} 58 \%, \mathrm{~T}=\mathrm{B} 37 \%$, $\mathrm{T}<\mathrm{B} 5 \%$; and correlated with high ( $\mathrm{HG}>=$ Gleason 7 ) vs low ( $\mathrm{LG}<=$ Gleason 6 ) tumor grade [T $>$ B $79 \%$ HG vs $43 \%$ LG, T $=$ B $21 \%$ HG vs $49 \%$ LG, T $<$ B $0 \%$ HG vs $8 \%$ LG; $\mathrm{p}<0.0001]$ and advanced (stage III,IV) vs early (stage I, II) tumor stage [T>B $80 \%$ adv vs $41 \%$ early, $\mathrm{T}=\mathrm{B} 18 \%$ adv vs $52 \%$ early, $\mathrm{T}<\mathrm{B} 2 \%$ adv vs $7 \%$ early; $\mathrm{p}<0.0001]$. Variable nuclear KDM6A (nKDM6A) immunoreactivity was noted for both the tumor and adjacent benign glands in 33/125 (26\%) cases as follows: $\mathrm{T}>\mathrm{B} 24 \%, \mathrm{~T}<\mathrm{B} 2 \%, \mathrm{~N}$ $74 \%$, and correlated with tumor grade [T>B $38 \%$ HG vs $14 \% \mathrm{LG}, \mathrm{T}=\mathrm{B} 4 \% \mathrm{HG}$ vs $1 \%$ LG, N 58\% HG vs $85 \%$ LG; $p=0.004$ ] and tumor stage [T>B $38 \%$ adv vs $13 \%$ early, $\mathrm{T}=\mathrm{B} 4 \%$ adv vs $1 \%$ early, $\mathrm{N} 58 \%$ adv vs $86 \%$ early; $\mathrm{p}=0.002]$. On multivariate analysis, advanced stage independently predicted biochemical disease recurrence $(\mathrm{p}=0.015)$. Conclusions: Cytoplasmic and nuclear KDM6A expression portends poor prognosis in PACs correlating with high tumor grade and advanced stage. These findings support the role of KDM6A as a potential therapeutic target in aggressive prostate cancers warranting further study.

\section{Prognostic Value of ERG Expression in Prostate Cancer}

$M$ Taris, C Debiais, J Irani, X Cathelineau, P Blanchet, G Fromont-Hankard. Universitary Hospital, Poitiers, France; Montsouris Institute, Paris, France; Universitary Hospital, Pointe à Pitre, France.

Background: TMPRSS2-ERG fusion resulting in strong ERG expression occurs in about $50 \%$ of prostate cancer $(\mathrm{PCa})$ in Caucasian patients, and has been suggested to be less frequent in African-American patients. TMPRSS2 expression, together with ERG in case of fusion, is activated by the androgen receptor (AR). The prognostic relevance of ERG expression in PCa remains to date controversial. We investigated ERG expression in all stages of PCa natural history, according to the ethnic groups, expression of AR, pT stage and Gleason score. Then, we aimed to evaluate the prognostic impact of ERG expression in clinically localized cancers (CLC) surgically treated and in castration-resistant cancers (CRPC).

Design: Expression of ERG and AR was evaluated by immunohistochemistry on tissue microarrays of normal prostate tissues $(n=57)$, high grade PIN (HGPIN) $(n=57)$, CLC $(n=299,185$ Caucasians and 114 Caribbeans), metastasis $(n=17)$ and CRPC $(n=41)$. Results: No expression of ERG was found in normal prostate tissues. In Caucasian patients, ERG expression significantly increased from HGPIN (17.5\% of cases), to CLC $(33 \%)$, then to metastasis $(53 \%)(\mathrm{p}=0.03)$. In CLC, ERG expression was significantly more frequent in pT3 (43\%) compared to pT2 $(27 \%)(\mathrm{p}=0.03)$. ERG was also expressed in CRPC in $29 \%$ of patients. In CLC, ERG expression was less frequent in Caribbeans compared to Caucasians $(11.5 \%$ versus $33 \%, \mathrm{p}=0.001)$. Expressions of $\mathrm{ERG}$ and AR were significantly correlated $(\mathrm{p}=0.001)$. In Caucasian CLC, ERG expression was significantly associated with a longer recurrence-free survival $(\mathrm{p}=0.02)$, after adjusting for preoperative PSA, Gleason score and pTNM stage. In CRPC, ERG expression was significantly associated with a longer overall survival $(\mathrm{p}=0.04)$.

Conclusions: Our results confirm the lower incidence of TMPRSS2-ERG fusion in PCa patients from African descent compared to Caucasians. The increased expression of ERG according to the stages of PCa natural history (from HGPIN to metastasis) strongly supports the concept that ERG target genes are involved in the migration of PCa cells. In contrast, we found a favorable prognostic impact of ERG expression both in term of recurrence-free survival in CLC, and in term of overall survival in CRPC. These results could be explained by the fact that ERG expression in PCa reflects (together with the presence of the fusion gene) the activation of AR, that could induce in CRPC a better response to androgen suppression.

\section{Endometrial Stromal Sarcoma (ESS) Involving the Urinary} Bladder: A Study of 6 Cases

$W$ Tian, $M$ Latour, JI Epstein. Johns Hopkins Hospital, Baltimore, MD; Centre Hospitalier de l'Université de Montréal, Montreal, QC, Canada.

Background: ESS involving the urinary bladder is very rare with no prior series.

Design: We identified 6 cases of low-grade ESS involving the bladder at our institution (1998-2013), of which 5 were consults.

Results: The median age was 50 (44 to 77). 1 patient presented with bladder serosal involvement at initial diagnosis of ESS. 5 presented 7-30 years (mean: 18) after a known diagnosis of ESS $(n=2)$ or remote history of hysterectomy with uncertain diagnosis $(n=3)$ with involvement of the bladder dome $(n=1)$, trigone $(n=2)$, diffuse involvement $(\mathrm{n}=1)$, and unknown $(\mathrm{n}=1) .2$ cases demonstrated worm-like infiltrating tumor nests classic of low-grade ESS with little stromal reaction with retraction artifact mimicking vascular invasion. The 3rd case originating from ovary, showed focal glandular differentiation in the bladder, resembling endometriosis. The 4 th-5th cases had abundant keloid collagen formation, arranged haphazardly or in a sunburst pattern. The 6th case showed primitive cells infiltrating entirely hyalinized stroma, after chemotherapy for a misdiagnosis of urothelial carcinoma. CD31 was negative in all cases, except for one case with obvious large vessel invasion. The differential diagnosis includes large nested variant of urothelial carcinoma, carcinoid tumor, synovial sarcoma, solitary fibrous tumor, Ewing's sarcoma/PNET, and endometriosis. CD10 was strongly positive in 5 cases and 1 case had very focal, moderate staining. ER and PR showed strong, diffuse staining in 5 cases and focal staining in 1 case. 1 case showed strong PAX-8 expression, and 2 cases showed p16 nuclear and cytoplasmic expression. CD56 showed weak to moderate staining in 4 cases. 1 case had diffuse and weak synaptophysin and 1 case has focal p63 positivity. CD99 was negative in all cases. The Ki-67 index was 1-10\% (mean $4 \%$ ). Mitotic count was $0-3 / 10 \mathrm{HPF}$ (mean $<1 / 10 \mathrm{HPF}$ ). Of the 5 patients with follow-up information, all are alive. 1 patient had metastases to the iliac lymph nodes and pelvic side wall at 1 year follow up, and had residual disease without progression by MRI 1 year after radiation. 2 patients were treated with Megace and 1 with Arimidex after surgery. Patients' follow up averaged 19 years (0-33 y) after initial diagnosis of ESS or hysterectomy and 3.5 years (0-11 yrs.) after bladder surgery.

Conclusions: ESS involving the bladder is extremely rare with a very long interval from onset to bladder involvement. In females, low grade spindle cell lesions involving the bladder should include ESS in the differential diagnosis.

1088 Sorbitol as a Novel Mechanism of Hypoxia-Inducible Factor (HIF) Pathway Activation in Clear Cell Papillary Renal Cell Carcinoma (CCPRCC)

SK Tickoo, AA Hakimi, J Xu, C-H Lee, Y-B Chen, S Stirdivant, B Neri, $R$ Wolfert, SW Fine, HAl-Ahmadie, A Gopalan, VE Reuter, JJ Hsieh. Memorial Sloan-Kettering Cancer Center, New York, NY; Metabolon, Inc, Durham, NC.

Background: CCPRCC is recognized as an entity distinct from clear cell RCC (ccRCC). Despite a lack of alterations in the $V H L$ gene or $3 p$ loss, the HIF pathway is noted to be 
activated in this tumor. To investigate differences between these two tumor types and potential mechanisms of HIF-activation in CCPRCC, we profiled the metabolome of CCPRCCs and compared them with ccRCCs and matched normal tissues.

Design: In collaboration with Metabolon Inc, metabolomic profiling was conducted on 9 CCPRCC and 9 non-tumor samples, along with 8 ccRCC profiled in an earlier study. Two additional tumors that were identified as outliers from previous profiling of ccRCC tumors were also included. Both these tumors, on pathological review of the slides, were found to be CCPRCC. Metabolite levels in the tumors and non-tumor frozen tissues were analyzed using three global profiling mass spectroscopy platforms. Welch's paired two sample $t$ tests were used to identify biochemicals that differed significantly between the tumor and normal tissue groups ( $p$ value $\leq 0.001$ ). Supervised clustering using random forest analysis was performed to identify metabolites that differentiated tumor from normal.

Results: Seven of the 9 newly profiled and 2 of 2 CCPRCC that had been profiled earlier (total, 9/11;82\%) displayed markedly elevated sorbitol levels (normalized sorbitol levels: range, 9.43-50.83; mean, 25.8; median, 18.92) relative to ccRCC tumors. Downstream fructose and sorbitol-6-phosphate metabolites were also elevated in CCPRCC. To assess the association of sorbitol with HIF, 3 renal carcinoma cell lines [ACHN (VHL wildtype), Caki-2 (VHL wildtype), and RCC4 (VHL mutated)] were cultured in the presence of $200 \mathrm{mM}$ sorbitol under either normoxic or hypoxic conditions. Sorbitol was found to induce HIF1-alpha protein over-expression under both normoxia and hypoxia in all 3 cell lines.

Conclusions: Clear cell papillary RCC is metabolically distinct from clear cell RCC, differentiated primarily by elevations in the sorbitol metabolic pathway metabolites. -Elevated intra-tumoral sorbitol levels are the likely mechanism of HIF pathway activation in these $V H L$ wildtype tumors.

1089 Chromosome 8 Alterations and PTEN Loss in Gleason Grade 3 Cores Predict the Presence of Unsampled Grade 4 Tumor: Implications for Active Surveillance

BJ Trock, RB Jenkins, JW Said, S Fine, B Knudsen, HL Fedor, B Gurel, TL Lotan, AM De Marzo. Johns Hopkins University, Baltimore, MD; Mayo Clinic, Rochester, MN; University of California, Los Angeles, CA; Memorial Sloan-Kettering Cancer Center, New York, NY; Cedars-Sinai Medical Center, Los Angeles, CA.

Background: A key eligibility criterion in many active surveillance (AS) programs is that the biopsy exhibit only Gleason pattern 3 (G3) for a Gleason score of 6 or less. However, $25-35 \%$ of biopsy Gleason 6 is upgraded to Gleason 7 or higher in the prostatectomy (RP) specimen. Thus, there is a great need for biomarkers that, when measured on G3 tissue in a Gleason 6 biopsy, can predict the presence of unsampled higher grade tumor in the whole prostate. We evaluated PTEN loss by immunohistochemistry (IHC), and PTEN deletion, chromosome 8q (MYC) gain and $8 \mathrm{p}(L P L)$ loss by fluorescence in situ hybridization (FISH) for their ability to predict unsampled G4 tumor.

Design: A tissue microarray (TMA) was constructed of RP tissue from 3 groups of patients ( $n=50$ per group) whose prostates exhibited only Gleason $3+3$, only $3+4$, or only 4+3 tumor, matched on age, year of RP, and race. In each patient, multiple cores sampled only from areas of G3 were evaluated for PTEN deletion by FISH, PTEN loss by IHC, and chromosome $8 \mathrm{p} / 8 \mathrm{q}$ alterations by FISH. Biomarker results were compared between Gleason 6 vs 7 tumors using conditional logistic regression.

Results: Patients underwent RP in 2001-2009, had median age 60, and median PSA 5.2; $63 \%$ of tumors were organ confined. In univariate analyses $8 \mathrm{q}$ gain (odds ratio $\mathrm{OR}=8.9$, $\mathrm{p}<.0001), 8 \mathrm{p}$ loss $(\mathrm{OR}=6.9, \mathrm{p}<.0001)$, PTEN loss by IHC $(\mathrm{OR}=5.7, \mathrm{p}=.025)$, but not PTEN deletion by FISH $(\mathrm{OR}=1.5, \mathrm{p}=.477)$ were significantly more common in $\mathrm{G} 3$ cores from Gleason 7 tumors than $\mathrm{G} 3$ cores from Gleason 6 tumors. In multivariable analyses, $8 \mathrm{q}$ gain $(\mathrm{OR}=6.2, \mathrm{p}=.002)$ and $8 \mathrm{p}$ loss $(\mathrm{OR}=5.2, \mathrm{p}=.0002)$ remained highly significant. At least one high risk biomarker (8q gain, $8 \mathrm{p}$ loss, PTEN loss, or PTEN deletion) was found in $35.7 \%$ of Gleason $3+3$ vs $77.1 \%$ of Gleason $3+4$ vs $91.3 \%$ of Gleason $4+3$ tumors, $p<.0001$. Adjustment for confounding factors did not change the results. Conclusions: Chromosomal 8q gains (MYC), 8p loss (LPL), and PTEN loss measured in Gleason G3 TMA cores strongly differentiate whether the core comes from Gleason 6 or Gleason 7 tumor. If validated in biopsy Gleason 6 cores to predict prostatectomy Gleason 7 tumor these biomarkers could facilitate safe selection of men for active surveillance.

1090 Pathologic Features in Radical Prostatectomy Specimens Associated with Biochemical Failure in Patients with pT2 Prostate Cancer and Positive Surgical Margin

P Troncoso, JN Nguyen, IN Prokhorova, X Wang, EM Li Ning Tapia, MF Achim, JW Davis, BF Chapin. The University of Texas MD Anderson Cancer Center, Houston, TX; The University of Texas at Houston Medical School, Houston, TX.

Background: While 3 prospective trials have demonstrated benefit from adjuvant radiation (XRT) after radical prostatectomy (RP) in patients (pts) with positive surgical margins (PSM), its use varies amongst physicians. Objective pathologic criteria are needed to identify pts at greatest risk of biochemical failure (BCF) post RP.

Design: Pts with pT2, pN0/Nx and a PSM were selected among pts who underwent RP between $2002-2010$. BCF was defined as 2 sequential PSA values of $\geq 0.2 \mathrm{ng} / \mathrm{ml}$ or any detectable PSA prompting XRT. Pts receiving adjuvant XRT or with $<12$ months follow-up were excluded. Parameters evaluated included zonal origin, tumor volume and Gleason grades (GG) of tumor focus/i at the PSM, location and length of PSM. Results: 205 pts met the criteria with a median PSA of $5.3 \mathrm{ng} / \mathrm{mL}$ and median followup of 64 months. The majority ( $65 \%$ ) were low clinical stage (cT1c), low/intermediate grade $(11 \% / 82 \%)$ and $90 \%$ had a single site of a PSM. BCF occurred in 47 pts for a 5 yr BCF free survival (BCFFS) of $69 \%$. PSM length was significantly associated with BCFFS $(\leq 1 \mathrm{~mm}$ vs $>1, p=0.02)$. Pts with GG 3 tumors were less likely to experience BCF (5 yr BCFFS-96\%) regardless of PSM length, while BCFFS for pts with GG
$>3$ tumors was significantly different based on the length of PSM ( $\leq 1 \mathrm{~mm}$ vs $>1 \mathrm{~mm}$, $\mathrm{p}=0.03)$. On multivariable analysis, length of $\operatorname{PSM}(\mathrm{p}=0.05)$ and $\mathrm{GG}(\mathrm{p}=0.007)$ remained independent predictors of BCF.

Conclusions: Length of PSM and Gleason grade were found to be independent predictors of BCF in pts with pT2 PCa. These parameters should be considered when evaluating patients for adjuvant radiation and in the stratification of patients for prospective clinical trials.

\begin{tabular}{|l||l|l||}
\hline Variable & HR $(95 \% \mathrm{Cl})$ & P-value \\
\hline Log (PSA pre-op) & $1.43(0.87,2.35)$ & 0.16 \\
\hline Clinical Stage $>=$ T2 $($ vs. T1) & $1.26(0.7,2.3)$ & 0.44 \\
\hline Length of PSM $>1 \mathrm{~mm}$ (vs. $<=1 \mathrm{~mm})$ & $1.94(1,3.7)$ & 0.05 \\
\hline Gleason Grade of CA focus $>3$ (vs. 3) at PSM & $7.07(1.7,30)$ & 0.007 \\
\hline
\end{tabular}

Multivariable Cox proportional hazards model for biochemical failure-free survival.

1091 FISH Analysis of 637 Prostate Cancers: Associations of PTEN Genomic Deletion with Higher Gleason Score and Biochemical Recurrence D Troyer, T Jamaspishvili, J Good, J Squire, CAnary Pathology Team Investigating Tissue Biomarkers (CAPIT). Eastern Virginia Medical School, Norfolk, VA; Queen's University, Kingston, ON, Canada; University of Sao Paulo at Ribeirao Preto, Ribeirao Preto, Brazil; CAnary Pathology Team Investigating Tissue Biomarkers (CAPIT).

Background: This multicenter study utilized the Canary Retrospective Prostate Cancer Tissue Microarray (TMA) cohort comprising detailed clinical and pathological information as well as long follow-up (Hawley et al. 2013).

Design: The analysis examined the clinical impact of losses of the entire PTEN (10q23.3, phosphatase and tensin homolog on chromosome 10) gene using a four color fluorescence in situ hybridization (FISH) deletion assay that included two closely linked flanking probes either side of PTEN optimized to minimize truncation or "falsedeletion" during scoring.

Results: Analysis of 637 prostate cancers on the TMA indicated that overall rate of PTEN deletion in this cohort was $118(18.5 \%)$. Hemizygous PTEN losses were present in 58/637 (9.1\%) of cancers, with homozygous PTEN deletion observed in 60/637 $(9.4 \%)$ tumours. A subset analysis was performed on 265 tumors which showed that the proportion of tumours with a homozygous deletion was enriched with higher Gleason (GS) scores (GS $\leq 6,7, \geq 8$, in $18 \%, 31 \%, 36 \%$, respectively). Moreover it was found that GS $3+4$ tumors had high frequency of hemizygous PTEN deletion compared to GS $4+3$ and GS $\geq 8$. In contrast, homozygous deletions were higher in GS $4+3$ tumors compared to lower grade tumors $(\mathrm{p}=0.015)$. In addition, we detected monosomy of Chr. 10 in $2 \%$ of tumors with high-grade prostate cancers. Further analyses comparing other parameters of progression, such as biochemical recurrence and pathologic stage are in progress on this cohort.

Conclusions: Collectively, our data indicates that PTEN genomic deletion may aid to differentiate less aggressive cancers ( $\mathrm{GS} \leq 6$ and $\mathrm{GS}=3+4$ ) from more aggressive ones $(\mathrm{GS} \geq 7,4+3)$ and the association of homozygous deletions with higher GS suggests that complete loss of PTEN determined by FISH and/or immunohistochemistry may be a valuable prognosticator to aid clinical management.

1092 Prognostic Impact of Intraductal Carcinoma and Large Cribriform Growth Pattern after Prostatectomy

$D$ Trudel, MR Downes, J Sykes, J Trachtenberg, T van der Kwast. University Health Network, Toronto, ON, Canada.

Background: Intraductal carcinoma (IDC) of the prostate is a distinct morphological entity associated with higher Gleason score and poor prognosis. The prognostic significance of large cribriform Gleason pattern 4 (LC) has not been previously investigated. The aim of our study was to determine the impact of IDC and LC on time to biochemical recurrence (bRFR) in a prostatectomy cohort.

Design: 285 prostatectomies (1999-2001) were reviewed, graded with the ISUP 2005 modified Gleason score (GS) and assessed for the presence of LC and IDC on H\&E. Median follow up was 130.6 months. Variables recorded included: age, prostate weight, Gleason score, presence of LC and IDC, surgical margins, extraprostatic extension and T-stage (TNM). A triple cocktail immunostain was used to differentiate LC from IDC. 39 patients were excluded from analysis due to lack of follow up data, a further 42 were excluded due to missing slides/blocks. The Kaplan-Meier (KM) method was used to estimate 5-year survival probabilities. Cox proportional hazards models were used to generate hazard ratios (HR) and $95 \%$ confidence intervals

Results: Median age was 62.4 years $(n=204)$. The GS were: 5 or less $(n=41), 20.1 \%$, Gleason $6(n=67), 32.8 \%$, Gleason $7(n=79), 38.7 \%$ and Gleason 8 or higher $(n=17)$, $8.4 \%$. Multivariable analysis of the entire cohort showed that $>0 \%$ of either LC or IDC ( (LC+IDC) $>0 \%$ ) had a highly significant prognostic effect on bRFR (HR 3.80, p $<0.0001)$ after adjusting for GS and T-stage. In the GS $7(3+4$ and $4+3)$ group, (LC+IDC) $>0 \%$ was a stronger prognostic factor than primary Gleason grade (HR 2.23, $\mathrm{p}=0.043$ vs HR $1.06, \mathrm{p}=0.88$ ). IDC alone indicated the worst prognosis compared to $\mathrm{LC}$ alone or LC and IDC combined in GS 7 cancers (bRFR $48.6 \%$ vs $92.3 \%$ vs $72.2 \%$, $\mathrm{p}=0.03$ ). GS 8 (and higher) cancers showed a trend towards lower bRFR with IDC $+/$ - LC but due to small numbers $(\mathrm{n}=17)$ was not statistically significant.

Conclusions: IDC is recognized as a poor prognostic indicator in GS score 6 and 7 $(3+4)$ prostatic adenocarcinoma. Our study identifies the negative prognostic impact of IDC in all Gleason score 7 carcinomas, in addition to highlighting the poor prognosis of the LC variant of pattern 4 . Our results show that the presence of $>0 \%$ of either LC or IDC is a more significant prognostic factor than either Gleason score or T stage in determining patient outcome and we would suggest including the presence of LC and IDC in the pathology report. 
1093 Gene Expression Signature for Neuroendocrine Prostate Cancer Identifies Additional Markers of Neuroendocrine Differentiation

$H$ Tsai, H-L Tan, J Hicks, $R$ Vessella, J Luo, TL Lotan. Johns Hopkins Medical Institutions, Baltimore, MD; University of Washington Medical Center, Seattle, WA. Background: Accurately identifying neuroendocrine (NE) prostate carcinoma (PC) has profound implications for prognosis and therapy of this aggressive disease. Currently, NEPC is identified morphologically or using IHC markers with limited sensitivity and specificity. Here, we used the $\mathrm{LuCaP}$ xenografts to derive a gene expression signature for NE prostate cancer in order to identify additional NE markers in prostate cancer. Design: Four LuCaP xenografts with NE differentiation were identified by expression of chromogranin and/or synaptophysin in the absence of androgen receptor and PSA (Zhang et al, 2013). Gene expression profiling (Agilent microarrays) and differential gene expression were performed to compare these $4 \mathrm{NE}$ tumors to $22 \mathrm{LuCaP}$ xenografts lacking NE differentiation. A set of differentially expressed genes was used as the basis for an NEPC signature, and a corresponding NE score was formed as the signed average expression of the signature genes.

Results: 988 out of 41,000 probes showed differential expression in NE LuCaP xenografts, with 842 differentially up-regulated and 146 down-regulated, after adjustment for multiple testing at an FDR rate of $\mathrm{q}=0.001$. Gene Ontology classification indicated neuronal differentiation as the most enriched biological process, and a set of the top probes was used to construct an NEPC gene signature. Applying this signature to expression data from an independent series of prostate cancer xenografts (Tzelepi et al, 2012) or a set of primary tumors and metastases (Grasso et al, 2012), correctly segregated large and small cell NE tumors from adenocarcinomas. The NEPC signature was more commonly present in metastases vs primaries, and metastases with a high NE score had decreased overall survival (104.4 vs $46.4 \mathrm{mo}, \mathrm{p}=0.02$ ). A subset of these metastases were not previously annotated as NEPC, suggesting that additional markers may be useful. Accordingly, and in line with a previous study (Lapuk et al, 2012), we found that targets of the transcription factor REST, a master repressor of neuronal differentiation, were enriched in the $\mathrm{LuCaP}$ NE signature and independently derived REST loss signatures were sufficient to segregate $\mathrm{LuCaP}$ xenografts with NE differentiation.

Conclusions: Using the LuCaP xenograft series, we have validated a NE expression signature for prostate cancer. This signature may be useful for identifying additional IHC markers of NE differentiation, such as loss of REST and upregulation of REST targets and validation of these markers is ongoing.

1094 Presence of Intraductal Carcinoma in Needle Biopsy Is a Significant Prognostic Parameter for Prostate Cancer in Radical Prostatectomy Patients

$T$ Tsuzuki, M Kato, K Kimura, R Ishida, N Sassa, Y Yoshino, $R$ Hattori, $M$ Gotoh Nagoya Daini Red Cross Hospital, Nagoya, Japan; Nagoya University Hospital, Nagoya, Japan; Nagoya Medical Center, Nagoya, Japan; Nagoya First Red Cross Hospital, Nagoya, Japan.

Background: Presence of intraductal carcinoma of the prostate (IDC-P) is an adverse prognostic factor of PSA failure for radical prostatectomy patients. Recently, we demonstrated IDC-P in radical prostatectomy predict progression-free survival (PFS) and cancer-specific survival (CSS). We aimed to evaluate whether IDC-P in needle biopsies is also an adverse prognostic parameter for PFS and CSS in radical prostatectomy patients. Design: We retrospectively evaluated 206 high-risk prostate cancer patients treated with radical prostatectomy between 1991 and 2005 in the hospitals that the authors were affiliated to and reviewed slides of prostatectomy specimens. Data on serum PSA level, biopsy Gleason score (bGS, $>8$ or not), cT stage (cT2 or more), presence of Gleason pattern 5 were analyzed. Patient characteristics were analyzed using Fisher's exact test. Multivariate Cox proportional hazard regression models were developed to predict PFS and CSS

Results: The median age of the patients was 69 years. The median follow-up period was 83 months. IDC-P component was detected in 102 patients. Forty-four patients showed clinical failure, and 20 patients died of the disease. Patients with IDCP showed a higher increased PSA level, higher increased bGS, and more advanced $\mathrm{cT}$ than those without IDC-P ( $p<0.0001)$. In univariate analysis, IDC-P $(\mathrm{p}<0.0001)$, cT $(\mathrm{p}<0.0001)$, bGS $(\mathrm{p}=0.0002)$, and presence of Gleason pattern $5(\mathrm{p}=0.004)$ were significantly associated with PFS; IDC-P $(p<0.001)$ and $\mathrm{cT}(\mathrm{p}=0.02)$ were significantly associated with CSS. In multivariate analysis, IDC-P ( $\mathrm{p}<0.0001$; hazard ratio (HR), 3.574) and $\mathrm{cT}(\mathrm{p}=0.004$; HR, 3.087) were significantly associated with PFS; IDC-P ( $p=0.001 ; H R, 8.405)$ and PSA level ( $p=0.0044 ;$ HR, 2.920) were significantly associated with CSS.

Conclusions: The presence of IDC-P in needle biopsy is a prognostic parameter for both PFS and CSS in radical prostatectomy patients. The presence of IDC-P in needle biopsy also can be a useful maker to predict patients' clinical outcome prior to radical prostatectomy.

1095 The Presence of Intraductal Carcinoma on Needle Biopsy and Radical Prostatectomy Specimens Is a Significant Prognostic Parameter for Patients Receiving Neoadjuvant Hormone Therapy

$T$ Tsuzuki, K Kimura, M Kato, R Ishida, N Sassa, Y Yoshino, $R$ Hattori, $M$ Gotoh. Nagoya Daini Red Cross Hospital, Nagoya, Japan; Nagoya Medical Center, Nagoya, Japan; Nagoya University Hospital, Nagoya, Japan; Nagoya First Red Cross Hospital, Nagoya, Japan

Background: Intraductal carcinoma of the prostate (IDC-P) is an adverse prognostic factor for prostate-specific antigen (PSA) failure in patients undergoing radical prostatectomy (RP). Recently, we demonstrated that the presence of IDC-P in both needle biopsy (NB) and RP specimens predict progression-free survival (PFS) and cancerspecific survival (CSS). We aimed to evaluate whether the presence of IDC-P similarly predicts PFS and CSS $<$ ins datetime="2013-10-01T19:22" cite="mailto:Byouri04" $><1$ ins $>$ after neoadjuvant therapy (NEO)

Design: We retrospectively evaluated 121 patients (median age, 68 years) with high-risk prostate cancer treated with RP after NEO between 1991 and 2005 in the hospitals that the authors were affiliated with. After reviewing slides of the NB and RP specimens, we divided these patients into 4groups as follows: (1) presence of IDC-P in both NB and RP specimens (Group A: 28 patients); (2) presence of IDC-P in only NB specimens (Group B: 12 patients); (3) presence of IDC-P in only RP specimens (Group C: 23 patients); and (4) absence of IDC-P in both NB and RP specimens (Group D: 58 patients). In addition, data on PSA level, biopsy Gleason score (bGS; $>8$ or not), and cT stage (cT2 or higher) were analyzed. Patient characteristics were analyzed using Fisher's exact test. Multivariate Cox proportional hazard regression models were developed to predict PFS and CSS.

Results: The median NEO and follow-up period was 6 and 83 months, respectively. Twenty-three patients experienced clinical failure (Group A, 13 patients; Group B, 4 patients; Group B, 3 patients; Group D, 3 patients) and 20 patients died of the disease (Group A, 9 patients; Group B, 2 patients; Group B, 1 patients; Group D, 0 patients). No patient demonstrated statically significant differences in PSA level or bGS (both $\mathrm{p}=0.30$ ). However, there was statically significant difference in cT stage between Group $\mathrm{B}$ and $\mathrm{D}(\mathrm{p}<0.001)$. There were statically significant differences in PFS between Group $A$ and $C(p=0.02)$, Group A and D ( $p=0.001)$, and Group B and D ( $p=0.006)$. Further, statically significant differences were observed in CSS between Group A and C $(p=0.02)$ and Group A and D $(\mathrm{p}<0.001)$

Conclusions: The presence of IDC-P in both NB and RP specimens is a prognostic parameter for PFS and CSS in patients undergoing NEO, and can therefore serve as a useful marker to predict clinical outcome.

1096 Incidence and Pathologic Features of Intraductal Carcinoma of the Prostate in a Contemporary, Prospective Cohort of Men Undergoing Radical Prostatectomy

KR Turner, AG Winter, C Friedman, JM Mosquera, MM Shevchuk, AK Tewari, BD Robinson. Weill Cornell Medical College, New York, NY

Background: Intraductal carcinoma of the prostate (IDC-P) is an increasingly recognized growth pattern of prostate cancer that has been associated with other poor prognostic features and overall worse outcomes. Van der Kwast et al demonstrated that the presence of IDC-P in biopsies from intermediate- and high-risk men undergoing radiation therapy predicted shorter times to biochemical recurrence $(\mathrm{BCR})$ and increased risk of metastasis. The true incidence of IDC-P, however, is still uncertain. Watts et al. recently reported the incidence of IDC-P to be $2.8 \%$ of all men undergoing prostate biopsy and $10.6 \%$ in the subgroup of men who were diagnosed with prostate cancer. Given that needle core biopsies only sample a fraction of the gland, the actual incidence of IDC-P may be higher. Indeed, several previous studies have reported IDC-P to be present in up to $74 \%$ of radical prostatectomy (RP) specimens. However, these studies were limited by their retrospective nature as well as their focus on high-risk prostate cancer patients. In this study, we sought to prospectively determine the incidence of IDC-P in a contemporary, unselected cohort of men undergoing RP.

Design: 893 consecutive men underwent RP by a single surgeon during the two-year period from January 1, 2011 to December 31, 2012. RP specimens were wholly embedded and evaluated prospectively for the presence of IDC-P, in addition to other standard pathologic variables, by three urologic pathologists.

Results: IDC-P was identified in $143(16 \%)$ of 893 RP specimens. The presence of IDC-P was associated with higher tumor volume (14\% vs. $10 \%)$, higher Gleason score (7.4 vs. 7.0), higher rate of non-organ confined disease ( $65 \%$ vs. $30 \%)$, and presence of lymph node metastasis ( $4 \%$ vs. $1.5 \%)(\mathrm{p}<0.001$ for all). Preoperatively, patients who were found to harbor IDC-P had a higher number of positive biopsy cores (5.3 vs. 3.9), higher percent core involvement ( $62 \%$ vs. $44 \%$ ), and higher biopsy Gleason score $(7.2$ vs. 6.8$)(\mathrm{p}<0.001$ for all $)$

Conclusions: In an unselected, contemporary cohort of 893 men undergoing RP, IDC-P was prospectively identified in $16 \%$ of the resection specimens. Recognition of IDC-P is important, as it is not an infrequent finding, and it has been shown to have prognostic value in the biopsy setting. In line with previous studies, our results showed that men who had IDC-P on RP were more likely to also have other poor prognostic features. Continued follow-up of this cohort is needed to determine if IDC-P may predict BCR or the development of distant metastasis.

1097 Characterization of ERG Immunohistochemistry (IHC) and ERG Fluorescent In-Situ Hybridization (FISH) in a Rapid Autopsy Cohort of Patients with Metastatic, Castration-Resistant Prostate Cancer

AM Udager, SA Tomlins, AM Chinnaiyan, R Mehra. University of Michigan Health System, Ann Arbor, MI; Michigan Center for Translational Pathology, Ann Arbor, MI. Background: Gene fusions involving the $E R G$ locus occur in nearly $47 \%$ of human prostate cancers $(\mathrm{PCa})$, and $E R G$ rearrangements can be detected with high sensitivity and specificity by FISH and IHC. Since ERG protein expression is androgen-dependent in $E R G$ rearrangement positive ( $E R G^{\mathrm{pos}}$ ) tumors, we sought to examine the concordance between $E R G$ FISH and ERG IHC in a cohort of patients with lethal, metastatic, castration-resistant $\mathrm{PCa}$.

Design: A tissue microarray comprised of rapid autopsy material from thirty patients with metastatic, castration-resistant PCa was stained with ERG antibody (EPR3864; Ventana Medical Systems, Tucson, AZ) and analyzed by FISH with $E R G$ locus-specific break-apart probes. ERG FISH was scored as positive or negative. ERG IHC was scored as negative (0), weak (1), moderate (2), or strong (3), and the percentage of ERG positive tumor cells was recorded (0-100); ERG endothelial cell staining was used as an internal control. An ERG IHC score (range 0-300) was calculated for each core as the product of the expression level and percentage of positive tumor cells. 
Results: Twenty-one patients were evaluable for both $E R G$ FISH and ERG IHC. Fifty-five tissue cores, representing thirty-five sites from twelve patients, were $E R G$ FISH negative, and forty-three tissue cores, representing twenty-three sites from nine patients, were $E R G$ FISH positive. All twelve $E R G$ FISH negative patients were negative for ERG IHC (score = 0). Two ERG FISH positive patients, including one with small cell carcinoma, were also negative for ERG IHC (score $=0$ ). Three $E R G$ FISH positive patients had at least one negative ERG IHC site but also showed weak ERG IHC expression at another site (score range $=5-70$ ). The remaining four $E R G$ FISH positive patients demonstrated weak to moderate ERG IHC expression at all sites (median score $=105$; score range $=5-220$ ). Overall, the majority of $E R G$ FISH positive patients demonstrated negative to weak ERG protein expression, as detected by IHC. Conclusions: These data demonstrate frequent discordance between $E R G$ FISH and ERG IHC in $E R G^{\text {pos }}$ lethal, metastatic, castration-resistant PCa, as a result of dysregulated androgen signaling in these tumors. This suggests that $E R G$ FISH may be the preferred method for detecting $E R G$ rearrangements in $E R G^{\mathrm{pos}}$ metastatic, castration-resistant PCa patients, especially during selection for targeted therapy or clinical trial enrollment.

1098 Utility of ERG Immunohistochemistry for Evaluation of Lymphovascular Invasion in Testicular Germ Cell Tumors

AM Udager, JB McHugh, J Siddiqui, X Cao, AM Chinnaiyan, R Mehra. University of Michigan Health System, Ann Arbor, MI; Michigan Center for Translational Pathology, Ann Arbor, MI

Background: Lymphovascular invasion (LVI) in testicular germ cell tumors (GCT) is a predictive factor for distant metastasis and an important variable in the GCT treatment algorithm. While pathologic evaluation of LVI in radical orchiectomy specimens can often be accomplished via routine hematoxylin and eosin (H\&E) staining, some cases present challenging diagnostic dilemmas. ERG expression by endothelial cells can be detected robustly by immunohistochemistry (IHC), thus we sought to assess the utility of ERG IHC for evaluation of GCT LVI in radical orchiectomy specimens.

Design: All available GCT radical orchiectomy specimens from 2003-2013 were identified retrospectively by searching the pathology records database of the University of Michigan Health System. H\&E slides from cases reporting positive or equivocal LVI were re-reviewed, and sections with positive or equivocal LVI foci were identified for immunohistochemical evaluation. "Off the top" sections from each paraffin tissue block were stained with ERG antibody. All foci were re-scored as positive, equivocal, or negative for LVI based on ERG endothelial cell expression.

Results: Thirty-six foci from twenty-five cases were selected for evaluation; twentythree (23) and thirteen (13) foci were positive or equivocal for LVI by H\&E staining, respectively. Histomorphology of H\&E positive LVI foci included embryonal carcinoma (17), seminoma (2), yolk sac (2), and choriocarcinoma (2). Of the H\&E positive LVI foci, twenty $(87 \%)$ were ERG IHC positive, three were lost for evaluation upon sectioning, and none were ERG IHC equivocal or negative. Histomorphology of H\&E equivocal LVI foci included embryonal carcinoma (7), seminoma (4), and choriocarcinoma (2). Of these $\mathrm{H} \& \mathrm{E}$ equivocal LVI foci, five (38\%) were ERG IHC positive, three (23\%) were ERG IHC negative, two were ERG IHC equivocal, and three were lost for evaluation upon sectioning. All four of the H\&E equivocal LVI seminoma foci were able to be reclassified by ERG IHC as either positive (1) or negative (3).

Conclusions: These retrospective data suggest a supportive role for ERG IHC in evaluation of GCT LVI in radical orchiectomy specimens. While providing an additional layer of confirmation in cases of $\mathrm{H} \& \mathrm{E}$ positive LVI foci, ERG IHC can also help distinguish positive from negative LVI in more than half of H\&E equivocal LVI foci.

1099 The Role of Morphometric Image Analysis in the Difficult Distinction between Urothelium with Reactive Atypia and Urothelial Carcinoma In Situ $T$ VandenBoom, B Bahar, KMudaliar, H Chen, GA Barkan. Loyola University Medical Center, Maywood, IL

Background: The recognition of urothelial carcinoma in situ (UCIS) is critical due to the high risk of progression if not properly managed. Difficulties may arise when trying to decide between reactive atypia (RA) and UCIS. In these situations, immunostains (IHC) are typically employed to aid in this differentiation. At times IHC may be difficult to interpret and also contribute to increased healthcare expenses and prolonged turnaround time. We aim to determine if morphometric image analysis software can objectively measure histologic features to aid in the difficult distinction between RA and UCIS. Design: We retrieved 60 bladder biopsy specimens which were separated into four groups (15 each) which included straightforward benign (group 1), RA which required IHC (group 2), UCIS which required IHC (group 3), and straightforward UCIS (group 4). These cases were reviewed by a genitourinary pathologist to validate the original diagnosis. All cases had concurrent cytology and follow-up specimens which were consistent with the biopsy diagnosis. The cases were photographed at the same settings and magnification (40X) using a high-resolution Nikon DS digital camera. The images were converted to grayscale and were analyzed using NIH ImageJ software. Nuclear area, pleomorphism, diameter, and circularity were analyzed. Statistical analysis was performed using MedCalc Version 12.7.4.

Results: Statistical analysis of the data revealed significant differences $(\mathrm{p}<0.05)$ between all four groups for all characteristics analyzed. Moving from group 1 to group 4, there was a progressive increase in nuclear area (square pixels), nuclear pleomorphism (standard deviation), and nuclear diameter (pixels). On the contrary, with 1.00 indicating a perfect circle, there was a progressive decrease in circularity throughout the four groups.

\begin{tabular}{|c|c|c|c|c|}
\hline GROUP & AREA & PLEOMORPHISM & DIAMETER & CIRCULARITY \\
\hline Benign (1) & 321.5 & 149.5 & 26.3 & 0.781 \\
\hline RA+IHC (2) & 384.2 & 191.6 & 29.6 & 0.754 \\
\hline UCIS+IHC (3) & 610.3 & 486.7 & 36.1 & 0.736 \\
\hline UCIS (4) & 743.6 & 565.3 & 39.0 & 0.716 \\
\hline
\end{tabular}

Addtionally, when comparing combined groups 1 and 2 (benign/reactive) with combined groups 3 and 4 (UCIS), significant differences $(\mathrm{p}<0.05)$ were seen for all characteristics measured.

Conclusions: Our results indicate that morphometric image analysis can objectively differentiate between RA and UCIS rather than using IHC. This could lead to decreased expenses, decreased turnaround time, and enhanced patient care overall. This method is relatively simple and therefore could be easily implemented into the daily practice of a pathologist.

1100 The Utility of GATA3 in the Diagnosis of Bladder Carcinoma with Variant Histology

L Verduin, C Heitz, MJ Mentrikoski, MR Wick. University of Virginia, Charlottesville, VA.

Background: The transcription factor GATA3 is a recently described biomarker that is highly expressed in bladder and breast carcinomas. Although it has been shown to be a sensitive marker of primary bladder carcinomas showing purely urothelial differentiation, its ability to distinguish primary bladder carcinomas with variant morphology remains incompletely studied. This study was designed to address whether GATA3 staining is retained in nonurothelial histologic variants of bladder carcinoma. Design: 88 cases of primary bladder carcinoma were retrieved from the institutional archives, and included the following histomorphologic types: 6 small cell, 12 sarcomatoid, 17 adenocarcinomas, 26 micropapillary, and 27 squamous cell carcinomas. A tissue microarray was constructed with four $0.6 \mathrm{~mm}$ cores per tumor. Normal urothelium was used for control ( $\mathrm{n}=8$ ). GATA3 (L50-823, Biocare Meidcal, Concord, CA) immunostaining was performed using standard techniques. Nuclear staining was considered positive and scored semiquantitively. Staining intensity was reported as weak, moderate, or strong.

Results: Only rare, moderate GATA3 positivity was seen in the primary squamous cell $(4 / 27,14.8 \%)$, and small cell variants $(1 / 6,16 \%) .35 \%$ of the adenocarcinomas showed weak to moderate staining (6/17), with an even distribution between primary adenocarcinoma and urothelial carcinoma with glandular differentiation. $66 \%$ of the sarcomatoid variants $(8 / 12)$ were positive with variable intensity and distribution. The majority of the micropapillary variants exhibited strong, diffuse positivity $(24 / 26,92 \%)$. Conclusions: Of the five variants, only the micropapillary and sarcomatoid variants exhibited consistent and strong immunostaining for GATA3. Although GATA3 has been shown to be a useful marker of urothelial differentiation, our results indicate that sensitivity is lost when primary bladder carcinomas with variant histomorphology are encountered. While the micropapillary variant did show retained GATA3 expression, the lack of expression in the other variants could suggest that these tumor types are derived from a different progenitor or via a different pathway, than classic urothelial carcinoma. Overall, caution should be utilized when interpreting GATA3 staining in the context of possible metastasis from primary bladder neoplasms with variant morphology, as well as secondary involvement of the bladder by adenocarcinomas of the colon or squamous cell carcinomas of the cervix.

1101 Investigating the Role of miR-199a-3p in Papillary Renal Cell Carcinoma Type 1

SJ Wala, JR Karamchandani, YY Youssef, SA Al-Haddad, A Evans, MAS Jewett, GM Yousef. The Keenan Research Center in the Li Ka Shing Knowledge Institute of St. Michael's Hospital, Toronto, Canada; University of Toronto, Toronto, Canada; Toronto General Hospital, Toronto, Canada; Princess Margaret Hospital, Toronto, Canada.

Background: Renal cell carcinoma is a heterogeneous disease that encompasses multiple subtypes. Papillary RCC (pRCC) is the second most common subtype, which can be further classified into two morphologically and molecularly distinct types: type 1 and type 2 . pRCC type 1 (pRCC 1 ) constitutes majority of pRCC cases. There is currently a gap in our understanding of the dysregulated pathways driving pRCC 1 . Therefore, the aim of this study was to understand the pathobiology underlining the tumorigenesis of pRCC 1 .

Design: We compared gene and miRNA expression levels between pRCC 1 and normal kidney. We performed target prediction and pathway analysis of the predicted targets of miRNAs dysregulated in pRCC 1 . We validated the aberrant expression of miR199a-3p in pRCC 1 by qRT-PCR using matched pRCC 1 and normal kidney tissues. The predicted interactions between miR-199a-3p and CAV2, ITGB8, MET and MTOR was validated in a cell line model.

Results: Using hierarchical clustering, we showed that normal kidney samples clustered separately from pRCC 1 based on gene and miRNA expression profiling. We identified a number of statistically significantly dysregulated $(P<0.05)$ genes and miRNAs in pRCC 1 compared to normal. Aberrantly expressed genes and miRNAs in pRCC 1 are enriched in the focal adhesion pathway, in addition to others. miR-199a-3p was shown to be significantly down regulated in pRCC 1 compared to normal kidney. Predicted gene targets of miR-199a-3p were experimentally validated using miRNA mimics and luciferase assay.

Conclusions: Our results show that the dysregulation of focal adhesion may have a significant role in the pathogenesis of $\mathrm{pRCC} 1$ and this may be, in part, a result of aberrantly expressed miR-199a-3p. An understanding of the pathways altered in pRCC 1 can help develop novel personalized therapy for this tumor subtype. 
1102 Increased Androgen Receptor Gene Copy Number Is Associated with TMPRSS2-ERG Rearrangement in Prostatic Small Cell Carcinoma

L Wang, SR Williamson, S Zhang, J Huang, R Montironi, XDu, DD Davidson, $M$ Wang, JL Yao, A Lopez-Beltran, AO Osunkoya, GT MacLennan, LA Baldridge, L Cheng. Indiana University School of Medicine, Indianapolis, IN; Fudan University Shanghai Cancer Center, Shanghai, China; Henry Ford Hospital, Detroit, MI; University of California at Los Angeles, Los Angeles, CA; Polytechnic University of the Marche Region (Ancona), Ancona, Italy; Pathline Laboratories, Ramsey, NJ; Cordoba University, Cordoba, Spain; Emory University, Atlanta, GA; Case Western Reserve University, Cleveland, OH

Background: Small cell carcinoma of the prostate (PSCC) is a highly aggressive malignancy that often develops in patients previously treated with hormonal therapy for metastatic prostatic acinar adenocarcinoma. The TMPRSS2-ERG gene rearrangement is highly specific for prostate cancer and shared by PSCC; however, the role of androgen receptor $(A R)$ gene alterations and its interaction with TMPRSS2-ERG rearrangement are incompletely understood in PSCC. We evaluated $A R$ gene copy number and AR protein expression in a large series of PSCC and correlated with TMPRSS2-ERG rearrangement status of the same tumors.

Design: Sixty-one cases of PSCC were examined for $A R$ gene copy number and TMPRSS2-ERG rearrangement by fluorescence in situ hybridization (FISH) and AR protein expression by immunohistochemistry.

Results: Of 61 cases of PSCC, 51\% (31/61) demonstrated increased $A R$ gene copy number (FISH+), 54\% (33/61) were positive for TMPRSS2-ERG gene fusion, and $38 \%(23 / 61)$ showed AR protein expression. Of the 31 AR FISH+ cases, 23 also showed TMPRSS2-ERG gene fusion, and 16 expressed AR protein. Of the 33 cases with TMPRSS2-ERG fusion, 28 were $A R$ FISH+ or expressed AR protein. Statistically significant correlations were observed between $A R$ gene copy number or AR protein expression and TMPRSS2-ERG gene fusion ( $P=0.001$ and $P=0.03$, respectively). Conclusions: High $A R$ gene copy number emerges during the development of PSCC, often in association with TMPRSS2-ERG rearrangement. This potential mechanism warrants further study. Improvement will come from understanding the biology of the disease and integrating new therapies into the treatment of this rare and aggressive tumor.

1103 Rosai-Dorfman Disease (RDD) of the Male Genitourinary Systema and Kidney: Analysis of 6 Cases

C-C Wang, AO Osunkoya, TO Al-Hussain, JI Epstein. Johns Hopkins Hospital, Baltimore, MD; Emory University Hospital, Atlanta, GA; King Faisal Specialist Hospital, Riyadh, Saudi Arabia.

Background: Although Rosai-Dorfman disease (RDD) most commonly occurs in lymph nodes, it can also affect various extranodal sites. Cases involving the genitourinary (GU) system have been reported but are extremely rare. Here we present 6 cases of RDD involving the GU system.

Design: Cases were collected from 3 institutions, the majority from one of the authors' consultation service.

Results: 6 cases were identified in the GU system, 3 each from the testis and kidney.

\begin{tabular}{||l||l|l|l|l|l|l||}
\hline $\begin{array}{l}\text { Case } \\
\text { No. }\end{array}$ & Age/Gender & Organ & Size $(\mathrm{cm})$ & $\begin{array}{l}\text { Organ } \\
\text { confined }\end{array}$ & Emperipolesis & $\begin{array}{l}\text { Lymphoplasmacytic } \\
\text { Infiltration }\end{array}$ \\
\hline 1 & $41 / \mathrm{M}$ & Testis & 1.5 & Yes & Marked & Moderate \\
\hline 2 & $47 / \mathrm{M}$ & Testis & 5.1 & Yes & Mild & Moderate \\
\hline 3 & $72 / \mathrm{M}$ & Testis & 4.3 & Yes & Mild & Marked \\
\hline 4 & $76 / \mathrm{M}$ & Kidney & 10.5 & No & Mild & Moderate \\
\hline 5 & $35 / \mathrm{M}$ & Kidney & 15.5 & No & Moderate & Moderate \\
\hline 6 & $67 / \mathrm{F}$ & Kidney & N/A & N/A & Moderate & Marked \\
\hline
\end{tabular}

There were 5 males and 1 female with a mean age of 56 (35-76). Average tumor sizes was: $3.6 \mathrm{~cm}(1.5-4.3)$ in the testis and $13.0 \mathrm{~cm}(10.5-15.5)$ for the kidney. One case was diagnosed via needle biopsy without subsequent nephrectomy, with all other cases having either nephrectomy or orchiectomy. All 3 renal cases showed typical morphology similar to nodal RDD with scattered foci of lymphocytic aggregation and in 2 cases there was infiltration of perinephric adipose tissue. In contrast, all the 3 testicular cases were organ confined although the tumors had ill-defined borders with entrapment of Sertoli-only seminiferous tubules, as well as an evenly distributed lymphoplasmacytic infiltration. Emperipolesis was scant in 2 testicular and 1 renal lesion. In 3 of the cases, RDD cells had focal spindle cell morphology. In all 6 cases, immunohistochemistry (IHC) for S100 showed strong reactivity in the lesional histiocytes and highlighted the emperipolesis. Of the 5 cases with clinical information, none had systemic symptoms or underlying hematological diseases.

Conclusions: Our 3 renal cases were similar in morphology to typical nodal RDD. The testicular cases had some different features including: (1) entrapment of seminiferous tubules with Sertoli-like changes and (2) more even distribution of lymphoplasmacytic cells. The rarity of this lesion in these sites, paucity of emperipolesis in some cases, spindled cell morphology of RDD cells, and large tumor-like lesions with extension into perinephric tissue all contributed to the difficulty in diagnosing RDD in the GU tract. IHC for S100 was helpful to verify the diagnosis.

1104 Are There Differences among the Pseudocapsule of Different Types of Small Renal Tumors? - Critical Appraisal of 178 Small Renal Neoplasms L Wang, J Feng, H Averaz, GN Gupta, MM Picken. Loyola University Medical Center, Chicago, IL.

Background: Small renal tumors are usually well circumscribed and surrounded by a peritumoral pseudocapsule. Hence, most recently, off-clamp tumor enucleation has been proposed for small, nonhilar masses. While the optimal surgical margin and the oncologic outcome continue to be contested, little data is available on the pathologic features of the pseudocapsule. This study aimed to critically analyze the thickness of the pseudocapsule and tumor extrapseudocapsular extension (EPE) among different types of renal neoplasm
Design: All cases of small renal tumors $(\leq 4 \mathrm{~cm})$ that were surgically removed by total (87) or partial (62) nephrectomy, or by enucleation (29) procedures during 20022013 were re-examined microscopically. Special attention was paid to pseudocapsule completeness, thickness and EPE. The data were analyzed according to the histological tumor types. Student t-test and Chi-square tests were used for statistical analysis. Results: A total of 178 surgically-removed, small, renal tumors were reviewed, including 118 clear cell renal cell carcinomas (CLRCC) $(66.3 \%), 33$ papillary (PAP) $(18.5 \%), 9$ chromophobe (CHR) $(5.1 \%)$ and 18 oncocytomas (ONCO) (10.2\%). CLRCC showed the thickest pseudocapsule (average $0.23 \mathrm{~mm}$ ), followed by CHR $(0.17 \mathrm{~mm})$ and PAP $(0.16 \mathrm{~mm})$. ONCOs had the thinnest peudocapsule with an average thickness of 0.09 mm(Fig.1). The EPE rate was the highest among CHR (44.44\%) and lowest among CLRCC (30.51\%), while PAP was in between (39.39\%) ( $>00.05)$. Most ONCOs had a poorly-developed pseudocapsule. While the tumor border was smooth, only $16.67 \%$ of tumors showed pseudoinfiltration $(\mathrm{p}<0.05)($ Fig.2).
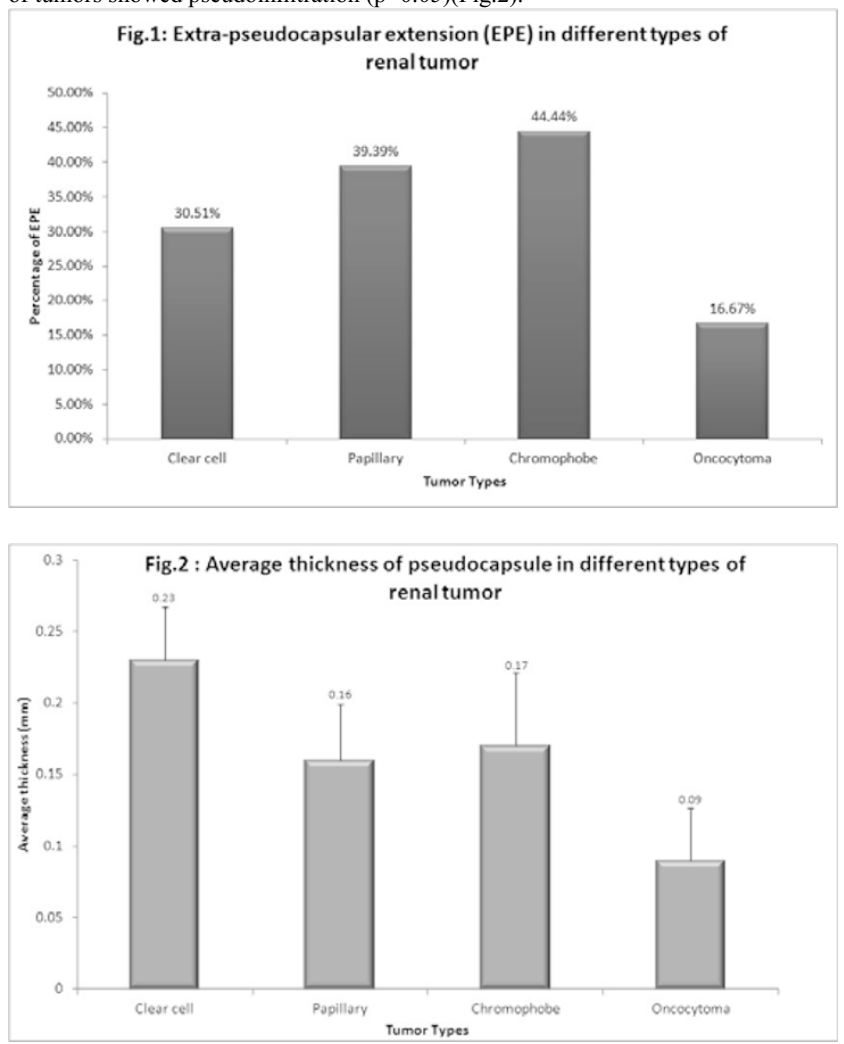

Conclusions: Among different types of RCC, CLRCC demonstrated the thickest pseudocapsule with the lowest EPE. PAP and CHR shared a similar thickness of pseudocapsule, while the latter had the highest ECE rate. Oncocytoma had the most poorly-developed pseudocapsule, but showed the least infiltrating growth pattern.

1105 ERG Cooperatives with SOX4 to Promote Epithelial-Mesenchymal Transition in Prostate Cancer

$L$ Wang, X Yang, B Han. Shandong University Medical School, Jinan, Shandong, China. Background: Approximately $50 \%$ of prostate cancer (PCa) patients in western countries harbor ERG rearrangement with concurrent ERG overexpression. Overexpression of SOX4 has been shown to play important roles in multiple cancers including PCa. However, the link between these two critical genetic aberrations was unclear.

Design: Fluorescence in situ hybridization and immunohistochemistry were utilized to detect ERG rearrangement and SOX4 expression. Cellular function was evaluated by transwell, wound healing assays, and cell adhesion assay, respectively. Interaction between ERG and SOX4 was arrayed by co-immunoprecipitation, Real-time PCR, western blot and SiRNA. Direct binding of ERG to the promoter of SOX4, as well as epigenetic modifications of their promoters after TGF- $\beta 1$ treatment was monitored by chromatin immunoprecipitation.

Results: ERG regulated SOX4 expression via binding to its promoter. Silencing both of them showed duplicate effects on restoring the epithelial characteristics, increasing cellular adhesion and decreasing capacity of cellular migration and invasion. ERG and SOX4 have cooperative roles in TGF- $\beta 1$-induced epithelial to mesenchymal transition (EMT) process. 


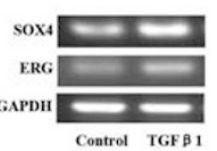

b

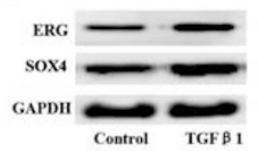

C
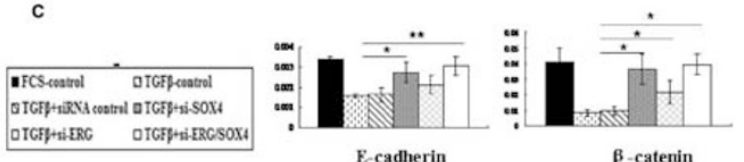

E-cadherin

B-catenin

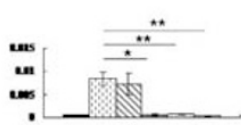

Snail

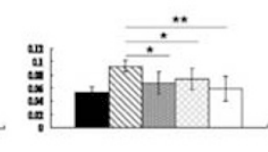

N-cadherin

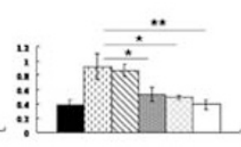

Vimentin

In addition, TGF- $\beta 1$ stimulation increased levels of chromatin marks associated with active genes (H3K4me3, H416ac), and decreased levels of repressive marks (H3K27me3) at their promoters. 5-aza and TSA treatment changed expressions of ERG and SOX4. Clinically, overexpression of SOX4 is associated with ERG rearrangement status in prostate cancer (Figure 2) and ERG+/SOX4+ defined a subset of PCa patients with poor prognosis.
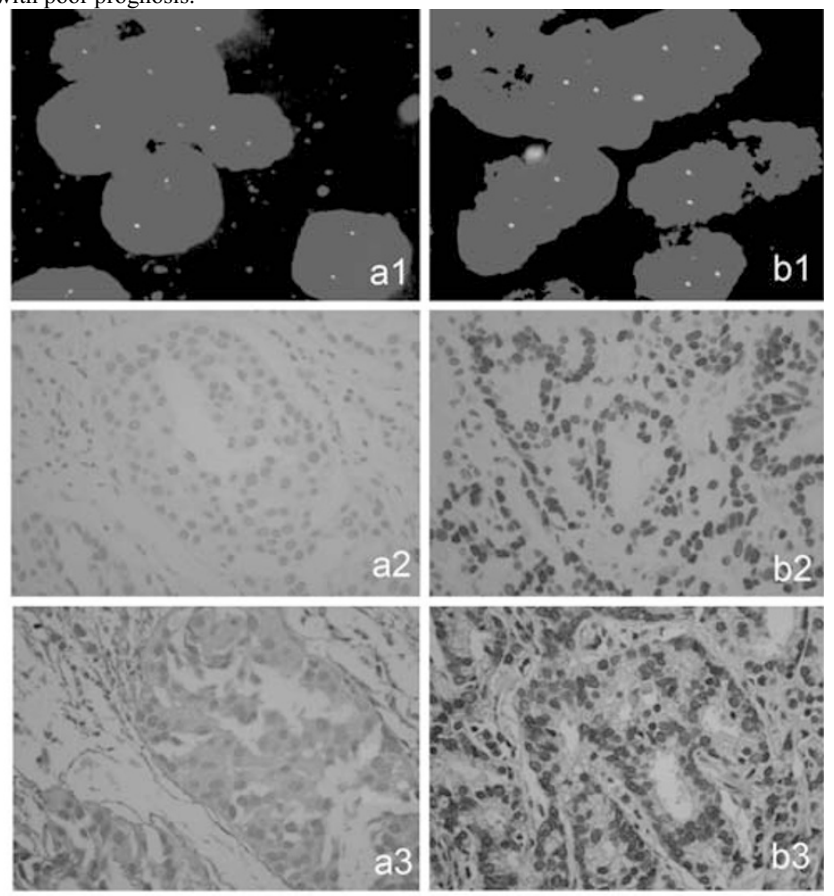

C

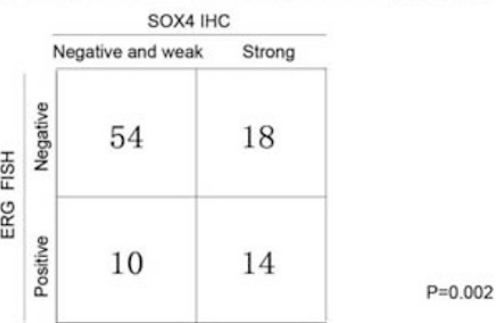

Conclusions: Our findings define a key role for ERG/SOX4 in the development of a subset of PCa and highlight the clinical importance of identifying molecularly defined tumor subgroups.

1106 Critical Pathologic Analysis of Pseudocapsule Margin after OffClamp Robotic Assisted Enucleation Partial Nephrectomy

L Wang, J Feng, H Alvarez, GN Gupta, MM Picken. Loyola University Medical Center, Chicago, IL.

Background: Off-clamp enucleation of small renal masses is gaining acceptance since it maximally preserves nephrons and allows for zero warm ischemia time. However, the optimal surgical margin for small renal masses is contested because of concern about the oncologic adequacy of enucleation margins. Our objective was to critically examine

the pseudocapsule of renal masses treated with robotic assisted off-clamp enucleation. Design: All consecutive renal enucleation specimens obtained during 2012-13 were reviewed. The tumor capsule was submitted in its entirety for microscopic examination. Particular attention was paid to pseudocapsule integrity and characteristics.

Results: A total of 34 enucleation specimens were reviewed, including 17 clear cell type renal cell carcinoma (RCC), 11 papillary RCC, 3 oncocytoma, 2 angiomyolipoma and 1 chromophobe RCC. Mean tumor size was $3.2 \mathrm{~cm}$ (range 1.2-8.5 cm). The pseudocapsule consisted of fibrotic tissue with adjacent compressed kidney parenchyma, frequently with sclerosed glomeruli and tubules, which creates a plane of dissection for enucleation. The average thickness of the pseudocapsule was $0.17 \mathrm{~mm}$ (range $0.01-0.55 \mathrm{~mm}$ ). When stratified according to tumor types, clear cell RCC had the thickest pseudocapsule $(0.21$ $\mathrm{mm})$, oncocytoma had the thinnest $(0.09 \mathrm{~mm})$, while papillary and chromophobe RCC were intermediate $(0.12 \mathrm{~mm} \& 0.14 \mathrm{~mm}$ respectively). The pseudocapsule was intact, with negative surgical margins in 29 cases $(85.3 \%)$. In 5 cases $(14.7 \%)$, tumor was focally seen at the inked margin ( 3 papillary RCC, 2 clear cell RCC) ranging from $<0.01-0.1 \mathrm{~mm}$. Arteries, with an outer diameter $\geq 0.2 \mathrm{~cm}$, were seen in $20 / 34(58.8 \%)$ specimens, including a total of 77 arteries, with an average arterial density of 2.26 / case. The average outer diameter of arteries was $0.30 \mathrm{~mm}$. Vascular compressions and prominent subintimal fibrosis contributed to lumen narrowing, with an average diameter of $0.08 \mathrm{~mm}$. Most arteries (73/77) ran parallel to the pseudocapsule, while 4 ran perpendicularly. Intra-pseudocapsular veins were largely collapsed or obliterated. Conclusions: In this study, the surgical margin was negative in $>85 \%$ of cases while, in $14 \%$, there was focal microscopic positivity. Clear cell RCC had the thickest pseudocapsule, while oncocytoma had the thinnest. Low arteriolar density and luminal narrowing may explain the low bleeding potential during the enucleation procedure. It is unclear whether focal margin positivity resulted from specimen handling, or represents a true feature. Its oncologic significance remains unclear and further studies are warranted.

1107 Sequencing Multifocal, Spatially Distinct Urothelial Carcinomas Demonstrates Tumor Clonality

JI Warrick, AK Cani, C-JA Liu, V Yadati, A Amin, A McDaniel, MJ Quist, CS Grasso, SA Tomlins. University of Michigan, Ann Arbor, MI.

Background: Urothelial carcinomas are frequently multifocal. Evidence for and against clonality in multifocal lesions has been documented. Recent exome sequencing studies of urothelial carcinomas have highlighted recurrent mutations involving the cancer associated genes TP53, RB1, HRAS, FGFR3, KRAS, and others. To our knowledge, no study has evaluated the clonality of multifocal urothelial carcinoma using comprehensive sequencing.

Design: We encountered a remarkable case of a man undergoing complete excision of the urothelium for five spatially distinct urothelial carcinomas arising in the bilateral renal pelvises, right ureter, urinary bladder, and prostatic urethra. All locations demonstrated non-invasive high grade papillary urothelial carcinoma; the right renal pelvis also showed underlying invasive urothelial carcinoma. Next generation sequencing was performed from DNA isolated from macrodissected FFPE sections. We sequenced the complete coding sequence of 400 cancer genes in each of the paired non-invasive and invasive urothelial carcinoma components (total $n=2$ ), non-invasive urothelial carcinomas $(\mathrm{n}=4)$, and normal kidney $(\mathrm{n}=1)$, utilizing Ion Torrent instrumentation, reagent kits, and software. High confidence variants identified in each of the 6 tumor specimens were filtered against the matched normal to identify somatic variants in each tumor sample. Copy number was estimated from normalized read counts. Identified mutations were confirmed by PCR.

Results: We sequenced the seven samples to an average depth of $333 x$ coverage. Identical mutations in five genes were present in all six urothelial carcinomas: HRAS (Q61L), FLT4 (Q827X), MLL2(Q3867fs), NTRK3 (T332M), and PIK3CA(H1047L). Compared to the overlying non-invasive tumor, the invasive carcinoma also demonstrated mutations in $C I C(\mathrm{~F} 1493 \mathrm{~L})$ and MYH9 (E1115K and E1244K). Only 5 unique mutations were seen in the remaining bladder tumor, ureter tumor, and left renal pelvis tumor. Copy number analysis supports clonal origin with broad gain of chromosome 8 in all six tumors.

Conclusions: The finding that all urothelial carcinomas in this case had identical mutations in several genes, and all had chromosome 8 copy number gains, are consistent with clonal origin for all tumors. Mutational profiles between the adjacent invasive and non-invasive components were highly similar and no candidate driver of the invasive component was identified. This approach may be used to assess the clonal relationship of synchronous and metachronous tumors, which are common in urothelial carcinoma.

1108 Investigation of Bladder Tumor Grade Heterogeneity Utilizing p53 and MIB-1

B Weening, MR Downes, KM Treurniet, TH van der Kwast. University Health Network, Toronto, ON, Canada; Erasmus University Medical Center, Rotterdam, Netherlands; Leiden University Medical Center, Leiden, Netherlands.

Background: The prognosis of patients with non-muscle invasive bladder cancer (NMIBC) may vary according to the degree of tumor differentiation. A proportion of NMIBC display grade heterogeneity: predominantly low grade with focal high grade areas. We hypothesize that NMIBC with heterogeneity represent tumors showing the process of grade progression over time. The aim of our study was to determine the degree of correlation between morphology in NMIBC and immunohistochemical markers of progression.

Design: A retrospective, 10 year search of the laboratory information system identified 28 NMIBC with grade heterogeneity. 11 pure low grade NMIBC were used as controls. The H\&E slides were reviewed, scanned and annotated (Omnyx ${ }^{\mathrm{TM}}$ VL4, [40x] magnification, yielded images of $0.1375 \mu \mathrm{m} / \mathrm{pixel}$ ) for low and high grade areas, using 
the WHO 2004 classification. The regions of interest were subsequently assessed by immunostaining for p53 and MIB-1 (cut-off values respectively 40 and 20\%). For all cases, clinical follow-up was recorded.

Results: MIB-1 showed low expression in all 11 control specimens. High expression of MIB-1 in low and high grade areas was observed in 12/28 and 22/28 cases, respectively. p53 was low in the low grade areas in $27 / 28$ cases. Of 8 NMIBC with high expression of p53 in the high grade areas (all with concurrent high levels of MIB-1), 5 showed distinct, high expression of MIB-1 in the low grade areas as well. In 12/28 cases the high grade areas had a morphological profile very distinct from the corresponding low grade areas. Nevertheless, half of these cases showed MIB-1 levels above the cut-off value in the low grade field. So far, $12 / 28$ patients (43\%) have recurred (mean follow up 42 months [3 - 118]), including 3 with high grade pT1 and concurrent CIS, 2 with CIS only and 2 with progression to either pT2 or metastatic disease.

Conclusions: Our preliminary results suggest that grade heterogeneity in NMIBC may represent a tumor in transition from low to high grade. MIB-1, but not p53 is an early marker of this grade progression. The results of this study justify the current practice of assigning "high grade" to bladder cancers exhibiting grade heterogeneity. Further work in progress aims to elucidate the underlying molecular mechanisms driving this process.

1109 Galectin-1 Has Potential Prognostic Significance and Is Implicated in Clear Cell Renal Cell Carcinoma Progression through the HIF/mTOR Signaling Axis

NMA White, O Masui, D Newsted, A Scorilas, AD Romaschin, GA Bjarnason, KWM Siu, GM Yousef. The Keenan Research Center in the Li Ka Shing Knowledge Institute of St. Michael's Hospital, Toronto, Canada; University of Toronto, Toronto, Canada; York University, Toronto, Canada; University of Athens, Athens, Greece; Sunnybrook Odette Cancer Centre, Toronto, Canada.

Background: Patients with metastatic clear cell renal cell carcinoma (ccRCC) have $<9 \%$ five-year survival rate. Metastatic RCC does not respond well to targeted therapy and patients eventually develop resistance. A better understanding of the molecular pathways of RCC metastasis is the basis for the discovery of novel prognostic markers and targeted therapies

Design: We investigated the biological impact of Gal-1 in RCC cell lines by migration and invasion assays. Effect of Gal-1 expression on the MAPK pathway was assessed by proteome array. We also assessed the prognostic significance of Gal-1 in primary RCC tissues using immunohistochemistry.

Results: Gain of function experiments showed that Gal-1 overexpression resulted in increased cellular migration. This was further validated by targeted knockdown of Gal-1, which resulted in significant reduction of RCC cell migration and invasion. In order to determine the downstream molecular pathways affected by Gal-1, we compared expression of the MAPK pathway before and after Gal-1 transfection. Gal-1 overexpression led to increased expression and phosphorylation of proteins involved in migration Of special interest is the activation of mTOR, which is a targeted therapy for metastatic RCC. We further validated the involvement of Gal-1 in the PI3K/Akt and mTOR signaling pathways by western blot. In addition, we examined HIF-1 $\alpha$ as a potential regulator of Gal-1. Induction of hypoxia led to a dose-dependent increase in HIF-1 $\alpha$, which correlated with a similar increase in Gal-1. We also provide evidence that miR-22 can target Gal-1 both directly and indirectly through HIF-1a. miR-22 was shown to be downregulated in RCC metastasis. Using ccRCC cell lines, we found that miR-22 overexpression resulted in a dose-dependent decrease in Gal-1 and HIF-1a proteins. Finally, we examined the utility of Gal-1 as a prognostic marker for ccRCC. High Gal-1 protein expression was associated with larger size and Grade III/IV tumors and shorter disease free survival. Using TCGA dataset, we found that high Gal-1 mRNA expression was associated with a significant shorter overall survival.

Conclusions: Our data suggest that Gal-1 can mediate cellular migration and invasion through the HIF-1 $\alpha$-mTOR signaling axis. Gal-1 represents a potential prognostic marker and therapeutic target for RCC.

\section{Pathologic and Clinical Evaluation of Tumor Recurrence in Partial} Nephrectomies

E Whittington, ON Kryvenko, G Campuzano-Zuluaga, S Umar, D Parekh, M Jorda. University of Miami/Jackson Memorial Hospital, Miami, FL.

Background: Partial nephrectomy has been increasingly used in management of renal cell carcinoma (RCC). The prognostic significance of positive surgical margin, tumor characterization, and patient properties are unclear in current literature. This study provides a single institution experience of tumor recurrence in partial nephrectomies. Design: Pathology data, patient characteristics, and tumor recurrence were reviewed in 463 consecutive partial nephrectomy cases from 459 patients diagnosed with conventional, papillary, or chromophobe RCC from 2003-2012. Univariant and multivariate statistical analysis was performed using Pearson $\chi^{2}$, Student T-test, and stratified analyses.

Results: There were 298 men and 165 women with median age of 58.9 (range: $21-85$ ). Tumors were 339 conventional RCC, 95 papillary RCC, 23 chromophobe RCC, and 6 mixed RCC ( 5 conventional/papillary RCC and 1 conventional/chromophobe RCC). Mean tumor size was $3.76 \mathrm{~cm}$ (range: $0.4-12.5 \mathrm{~cm}$ ). Twenty-eight $(6 \%)$ had positive margin. There was a significant difference in margin status in gender $(P=0.02)$ and age $(P=0.004)$, but not tumor size $(P=0.19)$. Patients with positive margin, older age, or male gender were significantly more likely to have tumor recurrence (Table). $54.5 \%$ of males with positive margins had recurrence compared to $0 \%$ in females with positive margins (proportion difference $54.5 \%(95 \% \mathrm{CI} 37.6 \%-71.5 \%)$. Patients with papillary RCC and positive margins had a $100 \%$ recurrence compared to $19.4 \%$ in patients with nonpapillary and positive margins (proportion difference $80.6 \%$ (CI 75.4\%-75.4\%) (Table).

\begin{tabular}{|c|c|c|c|c|c|}
\hline Variable & N & $\begin{array}{l}\text { + Tumor } \\
\text { recurrence }\end{array}$ & $\begin{array}{l}\text { - Tumor } \\
\text { recurrence }\end{array}$ & Differences* & P-value** \\
\hline Age- mean (SD) & 462 & 66.7 & 58.4 & $8.27(3.5-13)$ & 0.001 \\
\hline Sex- no. (\%) & 463 & & & & \\
\hline Male & & $27(9.1)$ & $271(90.09)$ & $9.0(0.8-17.3)$ & $<0.001$ \\
\hline Female & & $1(0.6)$ & $164(99.4)$ & & \\
\hline $\begin{array}{l}\text { Margin status- no. } \\
(\%)\end{array}$ & 463 & & & & \\
\hline Positive & & $18(41.9)$ & $10(2.4)$ & $16.2(1.2-31.2)$ & 0.001 \\
\hline Negative & & $25(58.1)$ & $410(97.6)$ & & \\
\hline Diagnosis- no. (\%) & 463 & & & & \\
\hline PRCC & & $20(21.1)$ & $75(78.9)$ & $18.9(7.1-30.7)$ & 0.001 \\
\hline Not papillary & & $8(2.2)$ & $360(97.8)$ & & \\
\hline
\end{tabular}

Conclusions: Patients with papillary RCC and positive margins are more likely to recur than with any other type of RCC. Positive margin status showed a large effect size for recurrence of tumor and appears to be the best predictor for recurrence of tumor. Male patients with positive margins of partial nephrectomy are more likely to recur than females. These findings may validate the frequent use of intraoperative frozen section consultation for margin status in partial nephrectomy specimens.

1111 Renal Cell Carcinoma with Angioleiomyoma-Like Stroma: Clinicopathologic, Immunohistochemical and Molecular Features Supporting Classification as a Distinct Entity

SR Williamson, L Cheng, JN Eble, LD True, $M$ Wang, S Zhang, DJ Grignon. Henry Ford Health System, Detroit, MI; Indiana University School of Medicine, Indianapolis, IN; University of Washington, Seattle, WA.

Background: Rare renal epithelial neoplasms have been recognized to have an angioleiomyoma or leiomyoma-like proliferation of stromal smooth muscle; however, the nature of these tumors and their relationships to other renal cell carcinomas are poorly understood.

Design: We analyzed 19 such tumors for their clinicopathologic features, immunoprofile, and chromosome $3 p$ status by fluorescence in situ hybridization.

Results: Nine showed a homogeneous combination of features and were reclassified as renal cell carcinoma with angioleiomyoma-like stroma. These were composed of neoplastic glandular structures lined by cells with mixed clear, pale and eosinophilic cytoplasm forming occasional papillary tufts. The stroma resembled smooth muscle and often extended away from the epithelial component, entrapping perinephric fat or nonneoplastic renal elements. Immunohistochemistry showed the epithelium to have reactivity for: carbonic anhydrase IX, CD10, vimentin, cytokeratin 7 , cytokeratin $34 \beta E 12$, and PAX8 but not $\alpha$-methylacyl-coA-racemase. The stroma was immunoreactive for smooth muscle (smooth muscle actin $3+$, desmin $1+$, caldesmon $3+$ ) but not epithelial antigens. Neither component showed substantial labeling for HMB45, melan-A, cathepsin K or TFE3 protein. An interrupted, conspicuous layer of CD34-positive endothelial cells rimmed the epithelium, imparting a two-cell layer pattern resembling myoepithelial or basal cells. Chromosome $3 p$ deletion was uniformly absent. Follow-up was available for 3 patients, none of whom experienced malignant behavior. Ten tumors were excluded from this category and considered to be clear cell renal cell carcinoma with a reactive proliferation of smooth muscle $(n=4)$ or tangential sectioning of the pseudocapsule $(n=2)$, renal cell carcinoma unclassified $(n=3)$, or clear cell papillary renal cell carcinoma $(\mathrm{n}=1)$.

Conclusions: In summary, renal cell carcinoma with angioleiomyoma-like stroma is a distinct neoplasm with characteristic morphologic, immunohistochemical and molecular features, unrelated to clear cell renal cell carcinoma. The immunoprofile overlaps partly with that of clear cell papillary renal cell carcinoma, though morphology and reactivity for CD10 are points of contrast.

1112 Molecular Characteristics of Urothelial Neoplasms in Children and Young Adults: A Subset of Tumors from Young Patients Harbors Chromosomal Abnormalities but Not FGFR3 or TP53 Gene Mutations SR Williamson, $M$ Wang, $R$ Montironi, JN Eble, A Lopez-Beltran, S Zhang, R Fan, L Wang, AO Osunkoya, MO Koch, L Cheng. Henry Ford Health System, Detroit, MI; Indiana University School of Medicine, Indianapolis, IN; Polytechnic University of the Marche Region (Ancona), United Hospitals, Ancona, Italy; Cordoba University, Cordoba, Spain; Fudan University Shanghai Cancer Center, Shanghai, China; Emory Universtiy, Atlanta, GA.

Background: Urothelial neoplasms in children and young adult patients are rare and hypothesized to have a lower rate of recurrence and progression than those of older adults. Due to this rarity, data regarding molecular abnormalities in these tumors are limited.

Design: We studied molecular characteristics of urothelial neoplasms from patients under age 30 years using UroVysion fluorescence in situ hybridization (chromosomes 3, 7, 17, and 9p21) and DNA mutational analysis for the FGFR3 and TP53 genes. Results: Seventeen tumors were identified in patients 6-26 years of age, including lowgrade papillary urothelial carcinoma $(\mathrm{n}=10)$, high-grade papillary urothelial carcinoma $(\mathrm{n}=5)$, urothelial papilloma $(\mathrm{n}=1)$, and papillary urothelial neoplasm of low malignant potential $(\mathrm{n}=1)$. No tumor demonstrated mutation of FGFR3 or TP53. Chromosomal abnormalities were detected only in patients 19 years of age or older: Two low-grade urothelial carcinomas had loss of 9p21 as a sole chromosomal abnormality and 3 highgrade urothelial carcinomas had other or multiple chromosomal abnormalities. Under age 19 years, no tumor showed molecular abnormalities with either method.

Conclusions: Our results support the idea that mutations of the FGFR 3 and TP53 genes are rare or absent in urothelial neoplasms of young patients. In contrast, chromosomal abnormalities detected by UroVysion fluorescence in situ hybridization are sometimes 
present in patients above 19-20 years of age. This finding supports the recently proposed hypothesis that an age of 19-20 years separates distinct molecular pathways of urothelial carcinogenesis

1113 Succinate Dehydrogenase-Deficient Renal Cell Carcinoma: Detailed Characterization of Clinicopathologic and Immunohistochemical Features of a Distinct Morphologic Variant

SR Williamson, JN Eble, MB Amin, NS Gupta, SC Smith, LM Sholl, MS Hirsch, JL Hornick. Henry Ford Health System, Detroit, MI; Indiana University, Indianapolis, IN; Cedars-Sinai Medical Center, Los Angeles, CA; Brigham and Women's Hospital, Boston, MA.

Background: Patients with germline mutations in succinate dehydrogenase (SDH) subunit genes are prone to develop paraganglioma, gastrointestinal stromal tumor, and rarely renal cell carcinoma (RCC). However, few SDH-deficient RCCs have been reported, and such tumors are not widely recognized. Therefore, we performed detailed evaluation of pathologic and immunohistochemical (IHC) features of SDHdeficient RCC.

Design: Renal tumors were identified based on distinctive morphology. SDH status was confirmed (loss of SDHB by IHC). Clinicopathologic and IHC features were examined using an extended panel of antibodies to renal tumor antigens.

Results: Nine tumors were identified from 8 patients (M:F 6:2), 22-72 years of age (median 36). Grossly, tumors were variegated tan or red-brown, sometimes cystic, $2-20 \mathrm{~cm}$ in diameter (median 4.25 ). One tumor was sampled by core biopsy. Fuhrman nuclear grade was $2(n=8)$ or $3(n=1)$. Stage was pT1a to pT2b. Two patients had paragangliomas, 1 had bilateral SDHB-deficient RCC, and 1 had contralateral oncocytoma (SDHB retained). One patient developed widespread metastases 16 years after nephrectomy and died of disease 6 years later. All tumors were composed of uniform eosinophilic cells containing vacuoles or flocculent eosinophilic inclusions. Architecture was predominantly solid or nested. Entrapped renal tubules were common $(n=7)$ and a tumor pseudocapsule was variably present $(n=4)$. By IHC, all had loss of SDHB and 1 had loss of SDHA. Tumors were diffusely positive for PAX8; reactivity for other renal epithelial antigens was limited and highly variable in extent: EMA (8/8 tumors), S100A1 (7/7), CD10 (2/7), AE1/AE3 (3/6), CAM5.2 (1/4), CK7 (1/9, focal) and AMACR (3/4, focal). EMA often showed a delicate apical staining pattern. Chromogranin, synaptophysin, cathepsin K, CA9, TFE3, RCC, and HMB45 were negative. KIT and vimentin highlighted intratumoral mast cells but were negative in tumor cells. Expression of HNF1B was retained.

Conclusions: SDH-deficient RCC is a unique neoplasm composed of uniform eosinophilic cells with cytoplasmic vacuoles and intratumoral mast cells, capable of progression over a protracted course. SDHA-deficient RCC can exhibit similar morphology. Other than SDHB loss, the IHC profile is not distinctive; however, select markers may be helpful to distinguish this tumor type from other oncocytic renal neoplasms.

1114 Development and Validation of a Formalin-Fixed Paraffin-Embedded Tissue-Specific MicroRNA Signature to Predict Clear Cell Renal Cell Carcinoma Metastasis and Prognosis

HWu, J Jin, XWu, A Li, S Pal, YLi, R Nelson, C Guo, Y Kim, J Wu, J Wang, K Haberman, $C$ Lau, W Chen, B Yuh. City of Hope, Duarte, CA

Background: Clear cell renal cell carcinoma (ccRCC) is the most common kidney malignancy. In the setting of metastasis, few patients achieve a durable remission with available therapies. RCC metastasis cannot be reliably predicted based on patients' clinical magnifications, pathologic findings or other currently available molecular tests. The early prediction of ccRCC metastasis may help to stratify patients for suitable clinical trials and to identify subsets of patients that stand to benefit from specific targeted therapies. For this purpose, we developed and validated a 4-microRNA (miRNA) signature to predict ccRCC metastasis and prognosis specifically using formalin-fixed paraffin-embedded (FFPE) clinical samples.

Design: (1) We first established two separate ccRCC FFPE tissue cohorts. The training cohort $(n=28)$ included localized (stage $I, n=13$ ) and metastatic (stage IV, $n=15$ ) ccRCCs. The testing cohort $(\mathrm{n}=222)$ included ccRCCs of stage I ( $\mathrm{n}=123)$, II $(\mathrm{n}=24)$, III $(\mathrm{n}=33)$ and IV $(n=42)$. All the testing cohort patients had been followed for at least 3 years if no metastasis developed. (2) We analyzed the expression of the 4 miRNAs (miR10b, miR-30b, miR-139-5p and miR-199b-5p) that are highly associated with ccRCC metastasis in the training cohort specimens by qPCR with miR-24 as normalization control and developed a mathematical formula (signature) to calculate the risk score and determine the risk status of ccRCC metastasis. (3) We measured the expression of the 4 miRNAs in the independent testing cohort samples and examined the correlation of the signature with the patients' clinical presentation and outcome.

Results: (1) We have developed a FFPE-tissue specific miRNA signature to be highly associated with ccRCC metastasis. (2) We have used the signature to predict the risk status of metastasis on the patients' primary nephrectomy specimens $(n=222)$, with the overall sensitivity and specificity of $70 \%$ and $76 \%$ (59\% and $76 \%$ for stage I and II, and $80 \%$ and $83 \%$ for stage III diseases, respectively). (3) We have found the signature to be highly associated with patients' cancer specific survival.

Conclusions: (1) We have developed and validated a FFPE tissue-specific miRNA signature that can predict ccRCC metastasis and prognosis using routine clinical samples. (2) The signature is ready for further large multi-institutional clinical cohort validation. (3) The signature can be used in the future as a routine molecular test for stratifying patients for more efficient follow-up management, suitable clinical trial studies and specific molecular therapies.
1115 Incidence and Genetic Characteristics of Clear Cell Tububopapillary Renal Cell Carcinoma

$W X u, F-M$ Deng, J Melamed, M Zhou. New York University Langone Medical Center, New York, NY.

Background: Clear cell tubulopapillary renal cell carcinoma (CCTPRCC) is a newly recognized histological subtype with distinct clinicopathological and immunohistochemical features. However, its genetic features remain largely unknown. In this study, we investigated its incidence and genetic characteristics.

Design: All partial and radical nephrectomy specimens from 11/1/2011 to 7/31/2013 at the authors' institution were reviewed for their diagnoses and pathological characteristics. The diagnosis of CCTPRCC was based on characteristic morphology and immunophenotype. The genomic alterations of CCTPRCC were investigated using whole-genome single nucleotide polymorphism (SNP) genotyping array. Genomic DNA was extracted from formalin-fixed, paraffin-embedded tumor and adjacent normal tissue from 11 CCTPRCC and hybridized to Affymetrix SNP 6.0 Array chips, which contained 1.8 million genetic markers, including 906,600 (single nucleotide polymorphism (SNPs) and 946,000 probes for copy number variation (CNV) detection. Chromosomal regions were marked as amplification or deletion when $>50 \%$ of the informative samples had a particular change.

Results: 260 cases of adult epithelial renal tumors were recorded during the study period. There were 11 cases of CCTPRCC ( $4.2 \%$ of renal epithelial tumors). It was the $5^{\text {th }}$ most common tumor after clear cell RCC $(132 / 260,50.8 \%)$, papillary RCC $(43,16.5 \%)$, oncocytoma $(35,13.5 \%)$ and chromomophobe RCC $(23,10.2 \%)$. Other tumors included $4(1.5 \%)$ multilocular RCCs, $3(1.2 \%)$ mucinous tubular spindle cell carcinomas, 1 (0.4\%) tubulocystic RCC, 1 collect duct RCC, 6 (2.3\%) unclassified $\mathrm{RCCs}$, and 1 metastatic lung carcinoma. For patients with CCTPRCC, male to female ratio was 2.7:1 (8/3), and mean age was 61.5 (40-78). All 11 CCTPRCC cases had cystic changes ( 9 predominant, 2 focal), and were stage pT1 (9 pT1a, 2 pT1b). SNP genotyping study identified 22823 genomic changes in CCTPRCC with 102 amplifications involving $2 \mathrm{q} 35,5 \mathrm{q} 13.2-13.3,7 \mathrm{p} 11.2$ and $11 \mathrm{q} 23.2-23.3$, and 16 deletions involving $1 \mathrm{q} 21.3$ and 3 p21.1. VHL locus (3p25.3) deletion was observed in 3 of 11 cases (27\%).

Conclusions: CCTPRCC ranks as 5th most common renal cell neoplasm (4.2\%) and is not as rare as initially thought, presenting as low stage (pT1) cystic lesions. It has a unique pattern of genetic changes, namely amplification of $2 \mathrm{q} 35,5 \mathrm{q} 13.2-13.3,7 \mathrm{p} 11.2$ and $11 \mathrm{q} 23.2-23.3$, and deletion of $1 \mathrm{q} 21.3$ and $3 \mathrm{p} 21.1$. VHL deletion (3q25.3) is detected in about one third of cases.

1116 Molecular Classification of Prostate Cancer with Family History Based on PTEN and ERG Gene Status

$W X u$, Y Wang, F-M Deng, XWu, L Tang, S Ubaradka, C Birch, J Melamed, M Zhou. New York University Langone Medical Center, New York, NY; Ventana Medical Systems, Inc, Tuscon, AZ.

Background: PTEN is the most frequently deleted tumor suppressor gene in prostate cancer (PCa). Alterations involving ERG gene is the most common and specific genetic change in PCa. Recent data demonstrate that stratification based on ERG and PTEN gene status significantly correlates with clinical outcomes. We investigated the ERG and PTEN gene status in PCa with family history and their prognostic significance.

Design: 170 PCa patients with and 171 patients without family history were included. A tissue microarray was used for chromogenic in situ hybridization detection of PTEN deletion and immunostain for ERG protein. Hemi- and homozygous deletion were considered positive for PTEN gene alteration. Positive ERG stain indicated ERG gene alteration.

Results: Patients with and without family history of PCa did not differ in the incidence of ERG, PTEN and combined ERG and PTEN gene alteration (Table 1). Alteration in both or one of ERG and PTEN genes was found in $7.9 \%$ and $47.4 \%$ patients (Table 1). Alterations in both ERG and PTEN genes were associated with significantly higher pathological stage and adverse clinical outcomes (Table 2).

\begin{tabular}{|c|c|c|c|c|}
\hline \multicolumn{2}{|c|}{ ERG and PTEN Gene Status } & \multirow{2}{*}{$\left.\begin{array}{l}\text { With Family History } \\
{[n(\%)]}\end{array}\right]$} & \multirow{2}{*}{$\begin{array}{l}\begin{array}{l}\text { No Family History } \\
{[\mathrm{n}(\%)]}\end{array} \\
866(50)\end{array}$} & \multirow{2}{*}{\begin{tabular}{|l} 
Total $[\mathrm{n}(\%)]$ \\
$164(48)$
\end{tabular}} \\
\hline ERG & Fusion & & & \\
\hline & Wild type & $92(54)$ & $85(50)$ & $177(52)$ \\
\hline \multirow[t]{3}{*}{ PTEN } & Wild type & $129(84)$ & $129(87)$ & $258(85)$ \\
\hline & $\begin{array}{l}\text { Hemizygous } \\
\text { deletion }\end{array}$ & $20(13)$ & $13(9)$ & $33(11)$ \\
\hline & $\begin{array}{l}\text { Homozygous } \\
\text { deletion }\end{array}$ & $4(3)$ & $7(5)$ & $11(4)$ \\
\hline \multirow[t]{3}{*}{$\begin{array}{l}\text { ERG + } \\
\text { PTEN } \\
\end{array}$} & Both wild type & $69(45)$ & $66(44)$ & $135(45)$ \\
\hline & Alteration in one & $72(47)$ & $71(48)$ & $143(47)$ \\
\hline & Alteration in both & $12(8)$ & $12(8)$ & $24(8)$ \\
\hline
\end{tabular}

$\mathrm{p}>0.05$ for all comparisons.

Table 2. Correlation of ERG/PTEN Status with Clinicopathological Parameters

\begin{tabular}{|c|c|c|c|c|}
\hline \multicolumn{2}{|l|}{ Parameters } & \multicolumn{3}{|c|}{ ERG/PTEN Gene Status [n (\%)] } \\
\hline & & $\begin{array}{l}\text { Both Wild Type } \\
\text { (A) }\end{array}$ & $\begin{array}{l}\text { Alteration in } \\
\text { One (B) }\end{array}$ & Alteration in Both (C) \\
\hline \multirow[t]{3}{*}{ Gleason Score* } & 6 & $54(40)$ & $67(47)$ & $6(25)$ \\
\hline & 7 & $68(50)$ & $62(43)$ & $14(30)$ \\
\hline & $\geq 8$ & $13(10)$ & $14(10)$ & $4(17)$ \\
\hline \multirow[t]{3}{*}{$\begin{array}{l}\text { Pathological } \\
\text { Stage** }\end{array}$} & $2 \leq$ & $90(67)$ & $108(76)$ & $9(38)$ \\
\hline & $3 a$ & $31(23)$ & $26(18)$ & $8(33)$ \\
\hline & $3 \mathrm{~b}$ & $14(10)$ & $9(6)$ & $7(29)$ \\
\hline \multirow[t]{3}{*}{ Disease Status*** } & No recur & $95(86)$ & $91(85)$ & $11(58)$ \\
\hline & PSA recur & $9(8)$ & $9(8)$ & $4(21)$ \\
\hline & $\begin{array}{l}\text { Never disease } \\
\text { free }\end{array}$ & $7(6)$ & 7 (7) & $4(21)$ \\
\hline
\end{tabular}

${ }^{*} \mathrm{p}>0.05 ;{ }^{* *} \mathrm{p}<0.001$ for $\mathrm{A}$ vs $\mathrm{C}$ and $\mathrm{B}$ vs $\mathrm{C} ;{ }^{* * *} \mathrm{p}=0.015$ for $\mathrm{A}$ vs $\mathrm{C}$, and $\mathrm{p}=0.02$ for $\mathrm{B}$ vs $\mathrm{C}$. 
Conclusions: The incidence of ERG and PTEN gene alterations is not different in PCa with and without family history. Alterations in both ERG and PTEN genes correlate with significantly advanced pathological stage and worse clinical outcomes.

1117 Biopsy Characteristics in Men with a Preoperative Diagnosis of High Gleason Score Adenocarcinoma (GS= 8-10) Predict Pathologic Outcome at Radical Prostatectomy (RP)

B Xu, E Scarlata, L Begin, A Spatz, L Salomon, G Ploussard, H Ye, A Aprikian, F Brimo. McGill University, Montreal, QC, Canada; Sacre Coeur Hospital, Montreal, QC, Canada; CHU Henri-Mondor, Créteil, France; Beth Israel Deaconess Medical Center, Boston, MA.

Background: Even if limited to one biopsy core, most urologists use the highest GS to guide therapy. This study aimed to evaluate the suitability of using biopsy characteristics to predict tumor characteristics at RP in men with high biopsy GS (BGS) cancer to better select men who will most benefit from RP.

Design: We retrospectively reviewed the biopsy and RP findings of 144 men with a BGS 8-10.

Results: 106 and 38 patients with a BGS 8 and 9-10 were included respectively. Cases with BGS 9-10 had significantly higher GS and more advanced $\mathrm{pT}$ and $\mathrm{pN}$ stage than those with BGS $8.48 \%$ of cases were downgraded to a final GS of 7 at RP, including $54 \%$ of BGS 8 , and $32 \%$ of BGS $9-10$ group. Overall $31 \%$ were found to have pT2 disease at RP. Multiple biopsy features, including the GS, the number of positive cores, the number of cores with high GS cancer, and the maximum volume of high-grade cancer per core (MVPC) consistently predicted final GS and RP tumor stage. Multivariate analysis showed that biopsy GS and MVPC were independent predictors of final GS, while MVPC was also an independent predictor for pT stage at RP. Compared with men with only one core of high GS cancer, patients with 2 cores of high BGS were associated with a significantly higher final GS and pTNM stage. Pre-operative PSA did not predict pathological findings at RP in our cohort.

\begin{tabular}{|c|c|c|c|c|c|c|c|}
\hline & Row total & RP GS 7 & RP GS $8-10$ & $\mathrm{P}$ & $\mathrm{T} 2$ & T3 & $P$ \\
\hline $\mathrm{N}(\%)$ & 144 & $69(48 \%)$ & $75(52 \%)$ & & $44(31 \%)$ & $99(69 \%)$ & \\
\hline $\begin{array}{l}\text { Pre-operative } \\
\text { PSA }\end{array}$ & $12.3 \pm 1.2$ & $\mid 10.8 \pm 1.8$ & $13.5 \pm 1.7$ & 0.264 & $9.2 \pm 1.9$ & $13.6 \pm 1.5$ & 0.103 \\
\hline Cores & $14.0 \pm 0.4$ & $13.0 \pm 0.6$ & $15.0 \pm 0.6$ & 0.034 & $13.0 \pm 0.7$ & $14.6 \pm 0.5$ & 0.113 \\
\hline Positive cores & $6.3 \pm 0.4$ & $5.3 \pm 0.5$ & $7.3 \pm 0.5$ & 0.008 & $4.7 \pm 0.7$ & $7.1 \pm 0.5$ & 0.004 \\
\hline High GS cores & $2.8 \pm 0.3$ & $1.8 \pm 0.4$ & $3.8 \pm 0.4$ & 0.001 & $1.6 \pm 0.5$ & $3.4 \pm 0.3$ & 0.001 \\
\hline MVPC & $57 \pm 3 \%$ & $47 \pm 3 \%$ & $66 \pm 4 \%$ & 0.001 & $46 \pm 5 \%$ & $52 \pm 3 \%$ & 0.002 \\
\hline
\end{tabular}

Values are expressed as mean \pm standard error of mean.

Conclusions: Patients with high BGS are not a homogeneous group in terms of local tumor characteristics. In addition to BGS (9-10 being worse than 8), other biopsy findings, especially the number of cores with cancer, number of cores with $\mathrm{HG}$ cancer, and MVPC are important predictors of findings at RP that should be incorporated in the clinical decision and treatment-planning.

1118

SDH-Deficient Renal Cell Carcinoma in Tuberous Sclerosis

Complex

$P$ Yang, $P$ Sadow, $L$ Cheng, $M$ Wang, $Y$ Xiao, $Z$ Jiang, E Oliva, AS Feldman, E Paul, EA Thiele, EP Henske, DJ Kwiatkowski, RH Young, $C-L W u$. Massachusetts General Hospital, Boston, MA; Indiana University School of Medicine, Indianapolis, IN; University of Massachusetts Medical School, Worcester, MA; Brigham and Women's Hospital, Boston, MA.

Background: Renal cell carcinoma (RCC) is a rare manifestation of tuberous sclerosis complex (TSC). Although heterogeneous histologic appearances have been reported in the literature, the full spectrum of morphologic and molecular features has not being fully elucidated. Negative SDHB expression by immunohistochemistry with a specific antibody has been shown to be highly correlated with mutations of SDH complex genes. Mutations of SDH genes are associated with hereditary paraganglioma and Carney-Stratakis syndromes and have only been described recently in limited cases of renal cell carcinomas. The morphological features of the SDH-deficient RCCs remain to be defined.

Design: We encountered 41 renal epithelial neoplasms from 18 TSC patients and analyzed the clinical, pathological and molecular features.

Results: Twenty-four (24) tumors in 9 patients with a distinct morphological and immunological profile were identified. Male-to-female ratio was 1:3. Mean age at diagnosis of renal tumor was 32.8 (24-48) years. Mean follow-up was 42 (9-96) months. All except one patient were alive without progression. Average size of tumor was $2(0.3-7) \mathrm{cm}$. Multiple tumors (3-15) were present in 5 patients, and 4 patients had bilateral disease. Concurrent angiomyolipoma was found in 5 patients. The tumors had prominent papillary architecture lined by cells with voluminous clear cytoplasm. Most $(83 \%)$ tumors were Fuhrman grade 1 or 2 with basally or centrally located nuclei and rare mitoses. Psammomatous calcifications were absent. Foamy histiocytes were occasionally seen in cystic fluid. Cytoplasmic inclusions were uncommon. Tumor cells showed strong diffuse labeling for CA-IX (100\%), CK7 (94\%), vimentin (88\%), CD10 $(82 \%)$, and were uniformly negative for succinate dehydrogenase subunit B (SDHB), TFE3 and AMACR. Gain of chromosome 7 and 17 were found in 2 tumors, whereas chromosome $3 p$ deletion and TFE3 translocation were not detected.

Conclusions: We identified a group of RCCs in TSC patients with distinct histological, immunohistochemical and molecular features, including uniformly negative SDHB expression. The findings suggest that loss of SDH complex, possibly due to genetic mutation, is involved in the tumorigenesis of RCCs arising in TSC disease.
1119 Extensive Transperineal and Transrectal Template-Guided Mapping Biopsy of the Prostate: Single Institution Five-Year Experience $O$ Yaskiv, TA Bhuiya, G Weiss. North Shore LIJ Health System, Lake Success, NY; North Shore LIJ Health System, New Hyde Park, NY.

Background: Extensive template-guided mapping prostate biopsy is considered in the high risk patients with increasing prostate specific antigen (PSA) and previously negative sextant biopsy. We describe our experience with extensive transperineal and transrectal template-guided mapping prostate biopsy in men in whom at least 2 previous sextant biopsy were negative or suspicious.

Design: Between January 2009 and September 2013, 30 men 49 to 72 years old (mean age 60.5) underwent extensive transperineal and transrectal template-guided mapping prostate biopsy at our institution. An average of 96.4 cores (range 31 to 170) were submitted. The cumulative number of biopsy cores in any patient was determined by prostate volume with more cores (doubled number) obtained from posterior and posterolateral regions. Indications for extensive template-guided biopsy were persistently elevated PSA in $100 \%$ of the cases and previous suspicious biopsy in 3 patients $(10 \%)$. All 30 patients had at least 2 prior biopsy of which $27(90 \%)$ were negative and $3(10 \%)$ were suspicious (atypical small acinar proliferation; ASAP).

Results: Adenocarcinoma was identified in 21 of the 30 patients (70\%). Biopsy revealed Gleason Score (GS) 6 to 8 . In 4 of the 21 patients (19\%) GS 6 only carcinoma was identified, with average core involvement of $17 \%$. Of the 3 patients in whom previous sextant biopsy showed ASAP extensive biopsy revealed adenocarcinoma in 2 patients (one patient: GS 6, less than 5\% involvement and other patient: GS 7 and GS 8 with $30 \%$ and $70 \%$ involvement, respectively). In 1 patient extensive biopsy revealed ASAP. Of the 4 patients undergoing radical prostatectomy (RP) at our institution, pathologically significant disease was identified in all 4 patients $(100 \%)$ with extraprostatic extension ( 4 patients; $100 \%$ ), positive surgical margin ( 3 patients; $75 \%$ ) and GS upgrade ( 3 patients (75\%): 2 patients GS $3+4=7$ in biopsy was upgraded to GS $4+3=7$ in RP specimen and 1 patient GS $3+3=6$ in biopsy was upgraded to GS $3+4=7$ in RP specimen).

Conclusions: Extensive transperineal and transrectal template-guided mapping prostate biopsy identifies significant prostate cancer in large percentage of men with previous multiple negative biopsies. This technique appears to enhance cancer detection in patients at high risk of prostate adenocarcinoma.

1120 Evaluation of Tissue PCA3 Expression by RNA In-Situ Hybridization in Prostate Core Biopsies Including Diagnostically Challenging Cases

JY Yoon, S Carskadon, JI Warrick, J Siddiqui, J Wei, SA Tomlins, A Chinnaiyan, $N$ Palanisamy, LP Kunju. University of Michigan, Ann Arbor, MI.

Background: PCA3 is a prostate-specific noncoding RNA overexpressed in $>90 \%$ of prostate cancer (Pca). Our group has developed a RNA-ISH (in-situ hybridization) assay to evaluate tissue PCA 3 and reported its expression in prostatectomies. This study evaluates PCA3 by RNA-ISH on core biopsies including diagnostically challenging lesions.

Design: We performed PCA3 RNA-ISH on a cohort of 100 consecutive cases $(n=243$ cores) over a 3 months period (Oct to Dec 2012) and a select cohort of biopsies (11 cases, $\mathrm{n}=68$ cores) enriched for high-volume Pca (median $60 \%, 40-90 \%$ Pca volume). In benign diagnoses, cores were randomly selected from both sides of prostate. In Pca cases, a core from each side was selected, generally representing the highest Gleason score. All cores with a diagnosis of HGPIN or atypia (ATYP) were selected. Finally, all cores requiring immunohistochemistry for diagnosis were selected. PCA3 RNA-ISH was scored as 0-4 (Warrick et al., Mod Pathol, 2013) and scores $\geq 2$ were considered positive. PCA3 expression was focal when staining was noted in $<50 \%$ of tumor cells in a core and heterogeneous when in multiple cores of Pca, some cores were negative while others were positive.

Results: PCA3 RNA-ISH was positive in the prospective cohort (median 2 cores, 2-5 cores) in $6.5 \%(16 / 243$ cores, $12 \%$ of cases) including $13 \%(9 / 68)$ of all Pca cores with focal expression in 66\% (6/9) of cores, $12.5 \%$ (4/32) HGPIN , 18\% (2/11) ATYP and $<1 \%(1 / 132)$ of benign cores . PCA3 expression was not seen in benign mimics such as adenosis and partial atrophy. Of the cohort enriched with high volume Pca (median 4 cores, $1-5$ cores), $18 \%$ (7/38 cores, $4 / 11$ cases) of cores were positive with PCA3 with focal expression in 30\% (2/7) cores and heterogeneous PCA3 expression in all cases. Overall, combining both cohorts, $15 \%$ (16/106) of all Pca cores were positive with PCA3. The overall sensitivity and specificity of PCA3 RNA-ISH for Pca detection across 281 cores was $15.1 \%$ and $96 \%$ respectively. The sensitivity and specificity for combined Pca, HGPIN and ATYP was $17.2 \%$ and $99.2 \%$ respectively.

Conclusions: PCA3 RNA ISH is specific for prostate cancer and HGPIN, and is not expressed in benign mimics and/or benign prostate glands. Interestingly, the sensitivity of PCA3 ISH for Pca on core biopsies is low ( $15 \%$ vs $54 \%$ in prostatectomies). This may be due to heterogeneity and focal expression of PCA 3 (also noted in our previous study). Further validation on a larger cohort to exclude selection bias is underway.

$1121 \quad$ Epidermoid and Dermoid Cysts of Adult Testis: A Contemporary Single Center Series with Long-Term Clinical Outcome

D Yu, K Trpkov, A Yilmaz. Calgary Lab Services, Calgary, AB, Canada; University of Calgary, Calgary, AB, Canada.

Background: Epidermoid cysts (EC) and dermoid cysts (DC) are unique and uncommon benign lesions of the adult testis. A painless intratesticular nodule with a characteristic 'onion skinning' cyst on ultrasound is the typical clinical presentation. The lack of intratubular germ cell neoplasia (ITGCN) is a critical histologic finding in differentiating these lesions from adult teratomas which are malignant with definite metastatic potential. To our knowledge this is the largest single center study with long-term clinical follow-up. Design: We identified 15 cases of EC and 2 of DC in 16 patients out of 611 consecutive 
germ cell tumors (GCT) in our testicular tumor data base during a 14-year period (10/1999 to 08/2013). All pathology reports and slides were reviewed. Follow-up was performed using information from pathology and clinical electronic records.

Results: The incidence of EC and DC was $2 \%$ and $0.3 \%$ respectively $(15 / 611$ and $2 / 611$ ). The mean patient age was 32 years (range 17 to 66 ). The right testis was more commonly involved by EC than the left $(9 / 15)$. Serum tumor markers were negative in all cases. One case had an unusual clinical presentation with a $10 \mathrm{~cm}$ cystic mass replacing the entire testis. $23 \%(4 / 17)$ of cases were managed conservatively by partial orchiectomy; intraoperative frozen section was performed in 3 cases. Median size was $1.7 \mathrm{~cm}$ (range 1.5 to 10 ). The classic gross findings of a unilocular cystic mass filled with keratinous material were found in all cases. The entire lesion was submitted in $94 \%$ of cases $(16 / 17)$. On histology all cases of EC showed a keratin filled simple cyst lined by benign squamous epithelium. DC cases further demonstrated a small additional non-cutaneous component. One case of DC was unusual as this patient had bilateral testicular nodules with DC in one testis and EC was found concurrently in the contralateral testis. All cases uniformly lacked ITGCN. There was no evidence of disease progression after a mean follow-up of 96 months (range 19.2 to 163.2) and all patients were alive.

Conclusions: Although uncommon, EC occurs more frequently than DC in the adult testis. An accurate diagnosis can be established using strict histologic criteria which required examination of the entire lesion in most cases. Conservative management should be considered in cases with a typical clinical presentation coupled with classic gross and histologic findings at intraoperative consultation and frozen section. The long term follow-up confirmed a benign clinical behaviour in both these entities.

\section{Urothelial Carcinoma of the Ureter: A Clinicopathological Study} of 97 Cases from a Single Institution

SS Zhang, GP Paner. University of Chicago, Chicago, IL.

Background: Upper urinary tract cancers arises anywhere from the renal pelvicaliceal system to the ureter, and are often lumped together in clinicopathological studies. Carcinomas from the ureter are relatively rare, with only few clinicopathological studies available focusing exclusively on tumors from this site. Herein, we present a series of ureteral urothelial carcinoma (UCa) treated at a single tertiary institution.

Design: From our surgical pathology files (July 2004 - July 2013), we identified 97 patients with ureteral UCa, which formed the basis of this study. 93/97 (96\%) of patients underwent tumor resections (e.g. ureterectomy or nephroureterectomy) and 4/97 (4\%) had biopsies.

Results: Patients included 69 males and 28 females $(\mathrm{M}: \mathrm{F}=2.5: 1)$ with age range of 42 to 89 years (mean 69 years old). These ureteral UCas included $9(9.3 \%)$ with squamous differentiation and $1(1 \%)$ with glandular differentiation. Most ureteral UCas $(85.2 \%)$ were high grade and only $14.8 \%$ were low grade. Overall tumor stage was pTa $(21.2 \%)$, pTis $(11.1 \%)$, pT1 (13.3\%) and pT2-pT4 (54.4\%). Only a subset of patients $(29 / 97,29.9 \%)$ presented first in the ureter and $27 / 97(27.8 \%)$ presented after an initial presentation of UCa in the bladder. In 40 cases $(41.2 \%)$, UCas were multifocal at the time of initial presentation, in which 31 cases $(32 \%)$ have concurrent UCa within the bladder. Mean interval for recurrence in ureter after bladder UCa was $30.1+/-7$ months. In $15(15.5 \%)$ cases, UCa recurred in the bladder or renal pelvis after initial presentation in the ureter with a significantly shorter mean interval of $11.5+/-2.2$ months $(\mathrm{p}=0.02)$. Conclusions: This study represents a large case series on ureteral urothelial carcinoma, summarizing their most updated histopathologic features and clinical data. Only a subset of primary ureteral UCas present as the initial presentation with some occurring as recurrence or concomitant to UCas elsewhere, mostly from the bladder. Unlike bladder $\mathrm{UCa}$, higher proportion of ureteral $\mathrm{UCa}$ are of high histologic grade and present in advanced stages. The interval from ureteral to bladder recurrence is significantly shorter than from bladder to ureteral UCa recurrence.

\section{Metastatic Renal Cell Carcinoma (RCC) to the Urinary Bladder:} A Report of 11 Cases

$M$ Zhang, W Cheuk, JI Epstein. The Johns Hopkins Hospital, Baltimore, MD; Queen Elizabeth Hospital, Hongkong, China.

Background: Metastatic RCC to the urinary bladder is rare with only case reports. Design: We identified 11 patients with metastatic RCC to the bladder in consultation (1998-2013). The referring diagnoses where there was diagnostic difficuly were: invasive high grade papillary urothelial carcinoma $(\mathrm{n}=2)$; rule out prostatic adenocarcinoma $(\mathrm{n}=1)$; and rule out urachal adenocarcinoma $(\mathrm{n}=1)$.

Results: There were 9 males and 2 females with a mean age of 66 (58-79). 4 patients presented with hematuria, 2 with urinary retention/obstruction, and 1 with bladder calculi. 4 were detected on routine surveillance cystoscopy. 9 patients had prior histories of RCC. The remaining 2 presented with metastatic clear cell RCC to the bladder and were subsequently found to have renal masses. The mean time between nephrectomy and metastasis to the bladder was $20.7 \mathrm{mos}$. (0-87 mos.). Of the 10 patients with nephrectomy, 7 cases were clear cell ( 2 with sarcomatoid features), 2 papillary, and 1 chromophobe. Of cases with available data, the primaries' Fuhrman grades were 2 $(\mathrm{n}=2), 3(\mathrm{n}=4)$, and 4 for the 2 with sarcomatoid features. In 6 cases, bladder RCC was polypoid and undermined overlying urothelium with extensive urothelial denudation. The chromophobe RCC metastasized with pagetoid spread to a pre-existing urothelial papilloma. PAX-8 IHC confirmed the diagnosis in 3 cases. 3 patients had no evidence of disease (7,9,13 mos.). 2 are alive with disease post-chemotherapy $(30,37 \mathrm{mos}$.). 4 died following therapy $(5,8,25,28 \mathrm{mos}$.), and 2 died without radiation or chemotherapy at 10 and 71 mos. with multi-organ metastases.

Conclusions: In rare cases metastatic RCC to the bladder may be the presenting site of disease and is usually clear cell RCC, and needs to be distinguished from other clear cell tumors in the bladder, such as urothelial carcinoma with clear cell features or clear cell adenocarcinoma. Polypoid growth of the RCC undermining the urothelium suggests that "drop metastases" down the ureter with bladder implantation may be the route of spread. This may explain the relatively long survival of several patients and that 4 patients have no other demonstrable metastatic sites other than the bladder or ureter. The one patient with chromophobe RCC, which typically has an excellent prognosis, involving the bladder via pagetoid spread is additional support for "drop metastases" as the mechanism in some cases. However, in other cases spread to the bladder was in the setting of metastases to other sites and these patients tended to die relatively shortly after their bladder metastases.

1124 High Frequency of TERT Promoter Mutations in Small Cell Carcinoma of the Urinary Bladder, but Not in Small Cell Carcinoma of Lung $X$ Zheng, J Zhuge, SM Bezerra, SF Faraj, E Munari, JT Fallon, XJ Yang, P Argani, GJ Netto, M Zhong. Westchester Medical Center/New York Medical College, Valhalla, NY; Johns Hopkins Hospital, Baltmore, MD; Northwestern University, Chicago, IL. Background: TERT promoter mutations were recently discovered in melanoma by next generation sequencing. Subsequently, several malignancies including urothelial carcinoma were also found to be associated with the same TERT promoter mutations. Small cell carcinoma (SCC) of the urinary bladder is a rare subtype with an aggressive clinical course. Despite the frequent occurrence $(\sim 70 \%)$ of TERT promoter mutations in urothelial carcinoma, the incidence of the mutations in SCC of the urinary bladder is unknown. In addition, as a potential molecular marker to distinguish SCC of the urinary bladder from small cell carcinoma of lung (SCLC) and other metastasis, this information may be clinically useful.

Design: We collected 10 cases of SCC of the urinary bladder ( 8 cases are primary SCC of the urinary bladder; 2 cases are from the same patient of both primary SCC of the urinary bladder and metastasis). We also included 20 cases of SCLC. In addition, several cases of non neoplastic tissue from the matched SCC of bladder patient were also studied. All tumor sections have been examined to make sure more than $20 \%$ of tumor content and confirm the diagnosis. Genomic DNA was isolated from FFPE tissue and a fragment of the TERT promoter (145 bp) was amplified by PCR. The TERT promoter mutations are determined by bi-directional Sanger sequencing.

Results: All (10) SCC of the urinary bladder bear TERT promoter mutation: C228T. None of SCLC or matched non-neoplastic tissue contains the TERT promoter mutations. Conclusions: We found a high frequency TERT promoter mutation in SCC of the urinary bladder, but not in SCLC. The findings further illustrate molecular differences between SCC of the urinary bladder and SCLC despite their shared morphologic and immunophenotypic features. The TERT promoter mutations could be of utility as a molecular marker to distinguish SCC of the urinary bladder from SCLC.

\section{Evaluation of CDX2 Expression in Urothelial Carcinoma of Bladder:} A Tissue Microarray Study

H Zhou, MR Schwartz, SS Shen. Houston Methodist Hospital, Houston, TX.

Background: CDX2 is a mammalian homeobox gene encoding a nuclear transcription factor which is limited to the intestinal epithelium. It is widely used as a marker for intestinal differentiation, particularly in the differential diagnosis of colorectal carcinoma from other carcinomas in the setting of metastasis. We recently encountered strong expression of CDX2 in metastatic and primary urothelial carcinomas, only some with glandular differentiation. Review of the literature has shown that there is variable expression of CDX2 in primary invasive bladder adenocarcinoma and urothelial carcinoma in situ with glandular differentiation. However, a comprehensive evaluation of a large cohort of bladder urothelial carcinoma including non-invasive papillary, primary invasive and metastatic urothelial carcinoma is not available.

Design: Expression of CDX2 in 224 bladder tumor samples was evaluated using tissue microarray and immunohistochemistry using a monoclonal antibody (clone CDX2-88 from BIOGENEX). The tumors represented in the TMA include non-invasive papillary (Ta) carcinomas $(n=100)$, invasive urothelial carcinomas $(n=94)$, and metastatic urothelial carcinomas $(n=20)$. Histological types include papillary and non-papillary urothelial carcinomas $(\mathrm{n}=203)$, and invasive non-urothelial carcinomas $(\mathrm{n}=21)$.

Results: Nineteen cases ( $8.5 \%$ ) had focal moderate (14 cases) or diffuse strong ( 5 cases) nuclear immunostaining for CDX2. The expression was detected in 11 non-invasive papillary urothelial carcinoma, 1 urothelial carcinoma in-situ, 6 invasive urothelial carcinomas and 1 metastatic urotheilal carcinoma. Of note, nine of 19 positive tumors had focal histologic glandular differentiation and the remaining 10 cases had no glandular differentiation. None of the non-urothelial carcinomas was positive for CDX2. Conclusions: CDX2 expression is detected in a subset of bladder urothelial carcinoma, including non-invasive papillary, invasive primary and metastatic urothelial carcinoma. Nearly half of the CDX2 positive tumors have histologic glandular differentiation. Most urothelial carcinomas with glandular differentiation have expression of CDX2. Our results suggest that CDX2 expression may be a marker of glandular differentiation in urothelial carcinoma. Importantly, in a metastatic setting, CDX2 immunoreactivity alone cannot be interpreted as evidence of gastrointestinal origin, given the therapeutic implication.

1126 The Necessity of Routine Analysis of Lymph Nodes in Periprostatic Fat Pad Following Robot-Assisted Radical Prostatectomy: An Institutional Study

HZhou, D Eun, E LLukani, VManucha. Temple University Hospital, Philadelphia, PA. Background: Periprostatic fat is dissected as a routine during radical prostatectomy but is not always sent for pathological examination. Based on the recent publications documenting presence of lymph nodes in periprostatic fat, we analyzed the rate of lymph node metastasis in this area to determine the necessity of obtaining pathologic analysis of this tissue on a regular basis. 
Design: From January 2011 to August 2013, 162 patients underwent robotic laparoscopic radical prostatectomy for clinically localized prostatic carcinoma at our institution. Periprostatic fat pads, yielded during defatting of the prostate, were dissected and sent to pathology for histopathologic examination in 133 cases. Clinical and pathological staging was recorded according to the 2009 American Joint Committee on Cancer (AJCC) criterion

Results: Of 133 patients whose periprostatic fat was examined, 32 (24\%) patients had lymph nodes in the periprostatic fat pads. Metastatic prostatic carcinoma to periprostatic lymph nodes was detected in 5 individuals (3.8\%). All 32 patients had bilateral pelvic lymphadenectomy. 3 of the 5 patients with positive periprostatic lymph nodes had no metastasis in pelvic lymph nodes, thereby upstaging 3 cases from T3N0 to T3N1. No relationship exists between the presences of periprostatic LNs and prostate weight, patient age, pathological staging or Gleason score. When compared to cases with tumor free periprostatic LNs, the tumor in cases with metastatic periprostatic LNs has following features: 1) higher postoperative Gleason score $(\mathrm{P}=0.01) ; 2)$ higher pathological staging $(\mathrm{p}=0.001) ; 3)$ higher rate of seminal vesicle invasion $(\mathrm{p}=0.01)$; 4) higher rate of extracapsular invasion $(\mathrm{p}=0.00001)$; higher rate of positive surgical resection margin $(\mathrm{P}=0.001)$

\begin{tabular}{|c|c|c|c|c|}
\hline & & $\begin{array}{l}\text { Pt with tumor free LNs in } \\
\text { periprostatic fat }\end{array}$ & $\begin{array}{l}\text { Pt with metastatic LNs in } \\
\text { periprostatic fat }\end{array}$ & P value \\
\hline Number of Patient & & 27 & 5 & \\
\hline \multirow[t]{3}{*}{ Age } & & & & 0.79 \\
\hline & mean & 60 & 59.2 & \\
\hline & range & $49-75$ & $50-62$ & \\
\hline \multirow[t]{3}{*}{ prostate weight } & & & & 0.33 \\
\hline & mean & 45.1 & 55.6 & \\
\hline & range & $23-123$ & $40-75$ & \\
\hline \multirow{5}{*}{\begin{tabular}{|l|}
$\begin{array}{l}\text { postoperative Gleason } \\
\text { score }\end{array}$ \\
\\
\end{tabular}} & & & & 0.01 \\
\hline & $3+3$ & 7 & 0 & \\
\hline & $3+4$ & 12 & 0 & \\
\hline & $4+3$ & 4 & 4 & \\
\hline & $>4+3$ & 4 & 1 & \\
\hline $\begin{array}{l}\text { Seminal vesicle } \\
\text { invasion }(+)\end{array}$ & & 6 & 4 & 0.01 \\
\hline $\begin{array}{l}\text { Extracapsular } \\
\text { invasion }(+)\end{array}$ & & 2 & 5 & 0.00001 \\
\hline $\begin{array}{l}\text { Surgical resection } \\
\text { margin }(+)\end{array}$ & & 7 & 5 & 0.001 \\
\hline \multirow{4}{*}{ Pathology stage } & & & & 0.001 \\
\hline & pT2 & 22 & 0 & \\
\hline & pT3a & 2 & 1 & \\
\hline & pT3b & 3 & 4 & \\
\hline
\end{tabular}

Conclusions: Despite the relative low incidence of positive periprostatic lymph node, routine pathological work up of periprostatic fat pads should be performed to guarantee adequate lymph node staging.

1127 Immunoexpression of Napsin A in Renal Neoplasms

$B$ Zhu, SM Rohan, D DeFrias, X Lin. University of Illinois at Chicago, Chicago, IL; Northwestern University, Chicago, IL.

Background: Immunohistochemistry (IHC) for Napsin A has been widely used to support a diagnosis of lung adenocarcinoma with high sensitivity $(93.9 \%)$. Previous reports have stated that Napsin A is also highly specific for lung adenocarcinoma (up to $94.7 \%$ ). However, a recent report demonstrated that Napsin A is expressed in papillary renal cell carcinoma (RCC). In this study, we evaluated the immunoexpression of Napsin A in renal neoplasms.

Design: Duplicate tissue microarrays (TMA) of 159 surgically excised renal neoplasms of various types were constructed. IHC for Napsin A was performed on TMAs with appropriate positive and negative controls. Tumors with more than $10 \%$ cells staining positive for Napsin A were considered as immunoreactive.

Results: See Table 1

Table 1. Expression of Napsin A in renal neoplasms.

Neoplasm

\begin{tabular}{|l|l|l|}
\hline Neoplasm & No. & Napsin A (\%) \\
\hline Acquired cystic disease associated RCC & 2 & 100.0 \\
\hline Chromophobe RCC & 45 & 11.1 \\
\hline Clear cell RCC & 23 & 43.5 \\
\hline Clear cell papillary RCC & 19 & 47.4 \\
\hline Metanephric adenoma & 3 & 100.0 \\
\hline Mucinous tubular and spindle cell carcinoma & 1 & 0.0 \\
\hline Oncocytoma & 23 & 56.5 \\
\hline Papillary RCC & 37 & 83.8 \\
\hline TFE/MITF RCC & 1 & 0.0 \\
\hline Urothelial carcinoma & 6 & 0.0 \\
\hline
\end{tabular}

Conclusions: Napsin A is expressed in various types of renal neoplasms with sensitivity up to $100.0 \%$. It is, sometimes, difficult to distinguish oncocytoma from chromophobe RCC on fine needle aspiration and/or needle core biopsy of a renal tumor. IHC for Napsin A may be useful in distinguishing oncocytoma from chromophobe RCC with positive labeling favoring oncocytoma. Based on our results, Napsin A is not specific for lung adenocarcinoma. When an metastatic carcinoma of unknown primary is positive for Napsin A, the differential diagnosis should include tumors of both renal and lung origin.

\section{Gynecologic and Obstetric Pathology}

1128 Recurrent Grade I, Stage la Endometrioid Carcinomas of the Uterus: Analysis of Pathology and Correlation with Clinical Data

SN Agoff. Virginia Mason Medical Center, Seattle, WA.

Background: Low-grade and low-stage endometrioid carcinoma of the uterus is thought to have an excellent prognosis in the vast majority of patients. However, despite the lack of myometrial invasion or superficial invasion, some patients develop recurrent disease, often in the vaginal apex. There is limited literature on these tumors, but recent analyses have implicated the pattern of myoinvasion as a prognostic factor. The emphasis of the current study is non-invasive or stage Ia, grade I endometrioid adenocarcinomas. Design: The pathology data base at Virginia Mason Medical Center (VMMC) was searched for cases of grade I endometrioid carcinoma from 1998 to 2012, along with prior biopsy diagnosis. Cases with concurrent ovarian and carcinoma were excluded as were cases with $50 \%$ or $>$ myometrial invasion. Pathological and clinical data was collected. Histological evaluation was performed, when possible, on either the original biopsy, hysterectomy and/or follow-up biopsies.

Results: At VMMC, there were 243 cases of grade I stage Ia endometrioid adenocarcinoma. Of these, 85 had lymph node dissections, 37 had omental and/or peritoneal biopsies. Peritoneal washes were performed on all patients and 12 were positive for tumor. Angiolymphatic invasion was present in 9 patients, lower uterine segment involvement was present in 5 (3 -epithelial only, and 2 - invasive) and endocervical gland involvement in 2 . From these, only 9 patients experienced recurrent disease $(4 \%)$, the majority in the vaginal cuff $(67 \%)$. Non-myoinvasive tumors accounted for 5 cases and 4 patients had superficial $(<50 \%)$ invasion. Angiolymphatic invasion was present in 2 patients, lower uterine segment invasion in one and one patient had a positive peritoneal wash. None of the patients had post-hysterectomy treatment. After recurrence, 7 patients had pelvic and vaginal radiation therapy, 2 chemotherapy (1 with radiation); 1 has not received therapy yet. Average follow-up time was 20 months ( 39 to 8 months), 2 were lost to followup. Six patients had NED, 1 died with disease. Conclusions: The majority of patients with grade I and stage Ia endometrial adenocarcinoma have an excellent outcome with no treatment. Rare cases have recurrence of disease. Treatment after recurrence in our study resulted in good clinical outcomes for patients where long term followup was available. Further analysis is need in order to determine why some patients recur after surgery while the majority do not.

1129 Uterine Serous and FIGO 3 Endometrioid Carcinomas: A MultiInstitutional Comparative Study of $\mathbf{3 3 5}$ Cases

OF Ahmed, YR Hussein, J Bennett, K Van De Vijver, S Thomas, B Alosh, E Abdulfatah, $D$ Schultz, S Bandyopadhyay, R Soslow, E Oliva, R Ali-Fehmi. WSU, Detroit, MI; Henry Ford Hospital, Detroit, MI; MGH, Boston, MA; MSKCC, New York, NY.

Background: Although uterine serous carcinoma (USC) and FIGO grade 3 endometrioid carcinoma(E3C) constitute a minority of all endometrial carcinomas, they account for a disproportionate number of deaths. The study aimed to compare clinicopathologic features and outcome of USC \& E3C.

Design: 335 endometrial carcinomas were identified from 4 institutions. Only E3C and USC reviewed and agreed on by 2 GYN pathologists(average of 4 slides/case) using WHO criteria were included in the study. 99 E3C and 167 USC were included in the final cohort. Clinicopathologic variables were analyzed, including patient demographics, tumor type, myometrial invasion, lymphovascular invasion(LVI), cervical involvement $(\mathrm{CI})$, lymph node(LN) status, FIGO stage, recurrence \& overall survival.

Results: Median age of patients with E3C and USC was 61 \& 69 yrs, respectively. Outer half myometrial invasion was identified more often in E3C compared to $\operatorname{USC}(\mathrm{p}=.001)$. Absence of myometrial invasion was seen more frequently in $\operatorname{USC}(\mathrm{p}=.002) . \mathrm{CI}(\mathrm{p}=.015)$, metastases to fallopian tubes $(\mathrm{p}=.001) \&$ recurrence $(\mathrm{p}=.001)$ were seen more frequently in USC. Although there was no significant difference in LVI, a higher number of positive LNs was identified in $\mathrm{E} 3 \mathrm{C}(\mathrm{p}=.001)$. Omental involvement was seen more in USC than $\mathrm{E} 3 \mathrm{C}(\mathrm{p}=.05)$. Early FIGO stage(I-II) was seen more frequently in E3C, whereas more cases of advanced stage (stage IV) were seen in $\operatorname{USC}(\mathrm{p}=.009)$. There was no statistical difference in overall survival between the 2 cohorts matched by stage $(\mathrm{p}=.264)$ 